\title{
Measuring and training of walking abilities in pediatric neurorehabilitation
}

Citation for published version (APA):

Ammann - Reiffer, C. (2018). Measuring and training of walking abilities in pediatric neurorehabilitation. [Doctoral Thesis, Maastricht University]. Datawyse / Universitaire Pers Maastricht. https://doi.org/10.26481/dis.20181114ca

Document status and date:

Published: 01/01/2018

DOI:

10.26481/dis.20181114ca

Document Version:

Publisher's PDF, also known as Version of record

\section{Please check the document version of this publication:}

- A submitted manuscript is the version of the article upon submission and before peer-review. There can be important differences between the submitted version and the official published version of record.

People interested in the research are advised to contact the author for the final version of the publication, or visit the DOI to the publisher's website.

- The final author version and the galley proof are versions of the publication after peer review.

- The final published version features the final layout of the paper including the volume, issue and page numbers.

Link to publication

\footnotetext{
General rights rights.

- You may freely distribute the URL identifying the publication in the public portal. please follow below link for the End User Agreement:

www.umlib.nl/taverne-license

Take down policy

If you believe that this document breaches copyright please contact us at:

repository@maastrichtuniversity.nl

providing details and we will investigate your claim.
}

Copyright and moral rights for the publications made accessible in the public portal are retained by the authors and/or other copyright owners and it is a condition of accessing publications that users recognise and abide by the legal requirements associated with these

- Users may download and print one copy of any publication from the public portal for the purpose of private study or research.

- You may not further distribute the material or use it for any profit-making activity or commercial gain

If the publication is distributed under the terms of Article $25 \mathrm{fa}$ of the Dutch Copyright Act, indicated by the "Taverne" license above, 


\section{MEASURING AND TRAINING OF WALKING ABILITIES IN PEDIATRIC NEUROREHABILITATION}
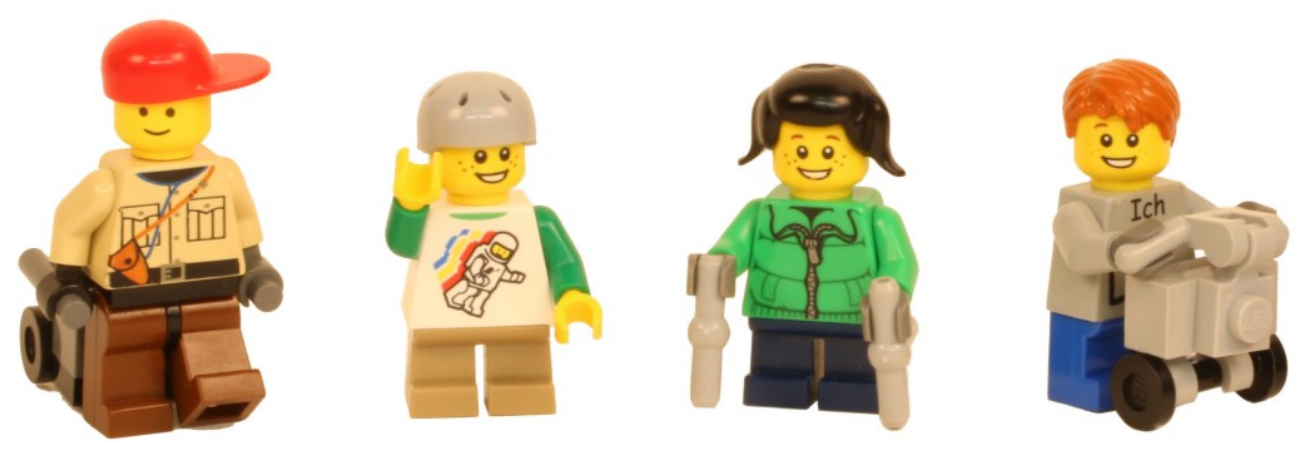

Corinne Ammann-Reiffer 


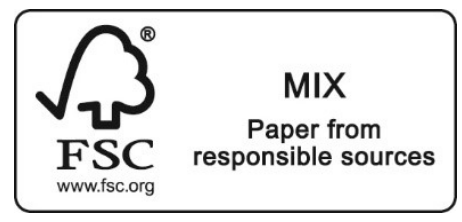

(C) Copyright C. Ammann-Reiffer, 2018

All rights reserved. No part of this thesis may be reproduced or transmitted in any form or any means, electronic or mechanical, including photography, recording or any information storage or retrieval system without permission in writing from the author, or, when appropriate, from the publisher of the publications.

Published by Datawyse - Universitaire Pers Maastricht

Printed by Gildeprint - Enschede

ISBN: 978-94-6323-352-1 


\section{MEASURING AND TRAINING OF \\ WALKING ABILITIES IN PEDIATRIC \\ NEUROREHABILITATION}

\section{DISSERTATION}

to obtain the degree of Doctor at Maastricht University, on the authority of the Rector Magnificus, Prof. dr. Rianne M. Letschert, in accordance with the decision of the Committee of Deans, to be defended in public on Wednesday, November 14 2018, at 10:00h

by

Corinne Ammann-Reiffer 


\section{Supervisor}

Prof. dr. R.A. de Bie

\section{Co-supervisors}

Dr. C. H.G. Bastiaenen

Prof. dr. H.J.A. van Hedel, University Children's Hospital Zurich, Switzerland

\section{Degree Committee}

Prof. dr. R.J. Vermeulen (chairman)

Prof. dr. K. Klingels, Hasselt University, Belgium

Dr. L.B. Mokkink, VU University Medical Center Amsterdam, the Netherlands

Dr. E.A.A. Rameckers

Prof. dr. R.J.E.M. Smeets

The research presented in this dissertation was partially supported by the UBS Donation by order of a client, the Stiftung Cerebral, and the Mäxi Stiftung, Zurich, Switzerland. 


\section{CONTENTS}

CHAPTER 1 General introduction

CHAPTER 2 Measurement properties of gait-related outcomes in youth 21 with neuromuscular diagnoses: a systematic review

CHAPTER 3 Concurrent validity of two gait performance measures in 59 children with neuromotor disorders

CHAPTER 4 Interrater reliability of two gait performance measures in children with neuromotor disorders across two different settings

CHAPTER 5 Responsiveness and minimal important change of two gait performance measures in children with neuromotor disorders

CHAPTER 6 Effectiveness of robot-assisted gait training in children with cerebral palsy: a bicenter, pragmatic, randomized, crossover trial (PeLoGAIT)

CHAPTER 7 Lessons learned from conducting a pragmatic, randomized, crossover trial on robot-assisted gait training in children with cerebral palsy (PeLoGAIT)

CHAPTER 8 General discussion

ADDENDUM Appendix

Summary

Zusammenfassung

Valorisation

Acknowledgments

About the author

List of publications 


\section{ABBREVATIONS}

3DGA

6MinWT

10MWT

10MWTss

10MWTm

$A D L$

$A \cup C$

COSMIN

$C P$

$D G O$

$D M D$

DS

$F A Q$

FMS

GMFCS - E\&R

GMFM D

GMFM E

GRS

ICF

ICF-CY 3-dimensional gait analysis

6-minute walk test

10-meter walk test

10-meter walk test self-selected speed

10-meter walk test maximal speed

Activities of daily living

Area under the receiver operating characteristic curves

COnsensus-based Standards for the selection of health

Measurement INstruments

Cerebral palsy

Driven gait orthosis

Duchenne muscular dystrophy

Down syndrome

Gillette Functional Assessment Questionnaire - walking scale

Functional Mobility Scale

Gross Motor Function Classification System - Expanded and Revised

Gross Motor Function Measure dimension D (standing)

Gross Motor Function Measure dimension E (walking, running, jumping)

Global rating scale

International Classification of Functioning, Disability and Health

International Classification of Functioning, Disability and Health for Children and Youth 
Incomplete spinal cord injury

MIC

Minimally important change

MICD

Minimum important clinical difference

MobQues28/47

28-item/47-item Mobility Questionnaire

MS

Multiple sclerosis

$P A$

Physical activity

PBWSTT

Partial body-weight supported treadmill training

$P D$

Parkinson's disease

PI

Principal investigator

PRECIS-2

PRagmatic-Explanatory Continuum Indicator Summary version 2

RAGT

Robot-assisted gait training

$R C A$

Rehabilitation center Affoltern am Albis of the University

Children's Hospital Zurich, Switzerland

$R C T$

Randomized controlled trial

$R O C$

Receiver operating characteristic

$r_{s}$

Spearman correlation coefficient

$S D$

Standard deviation

$T B I$

Traumatic brain injury

TUG

Timed Up \& Go Test

VR

Virtual reality

WeeFIM

Functional Independence Measure for Children

WHO

World Health Organization 

CHAPTER 1

\section{GENERAL INTRODUCTION}

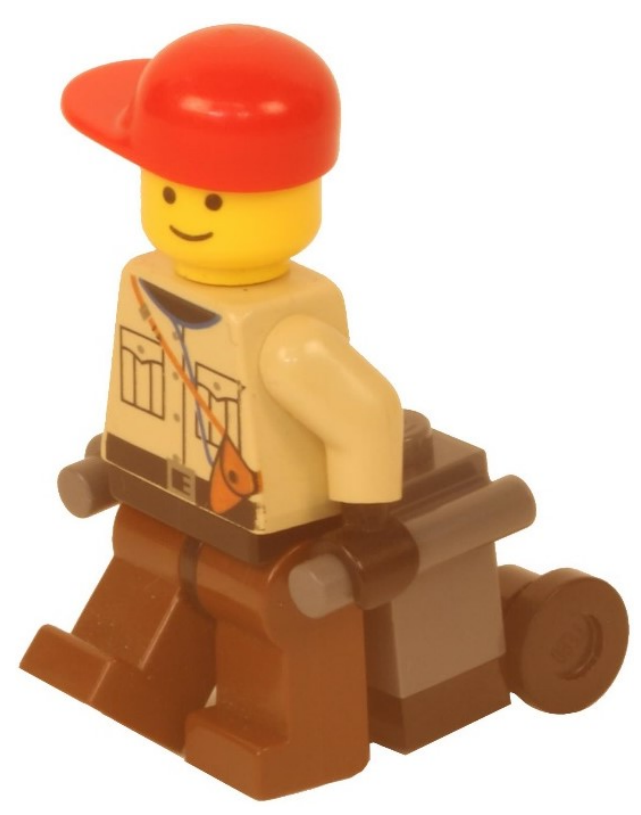




\section{BACKGROUND}

Mobility is defined as "the ability to move or be moved in one's environment with ease and without restriction." ${ }^{11}$ Adults might primarily associate personal mobility with getting around from one point to another, which is a relevant factor regarding physical independence as well as social participation. For children, it has other, more far-reaching meanings. With the achievement of independent mobility, young children begin to explore their surroundings actively and show an enhanced emotional expression and interaction with their caregivers. ${ }^{2}$

It allows them to decide for themselves where they want to go or what they want to explore, and thus interact with their environment. Hence, acquisition of independent mobility is linked to developmental progression in cognitive, social, motor, and language capabilities in young children. ${ }^{3-6}$

Independent mobility starts as soon as children discover their possibilities to move their body in any manner over the ground. However, parents often look anxiously forward to the moment their child starts to walk independently. For children, walking is not the objective, but a way of expanding and experiencing their environment. The onset of independent walking is known to change children's moods and their manner of interaction with other people. ${ }^{4}$ Within this context, social mobility is considered as the simultaneous engagement in self-directed locomotion and direct peer interaction. $^{7}$

When independent walking has become an everyday activity, motor and social experiences stimulate further locomotor skills and strategies. These abilities encourage children to master different terrains, speeds, and obstacles while walking, running, or jumping. ${ }^{8}$

Whereas reaching milestones of motor development is assumed to be normal in typically developing children, the range and variability of problems in motor development are huge in children with neuromotor disabilities. These children are often restricted with regard to independent mobility and daily walking activity. Their physical activity (PA) levels have been shown to be lower than in children who are developing typically; and PA levels subsequently decline the more children are disabled concerning their motor skills.9,10 Being carried or pushed in a stroller or 
wheelchair limits their abilities to explore and experience their environment actively. Hence, a substantial difference exists in social mobility between children with disabilities and those who are developing typically. ${ }^{7}$ This gap is evident in different contexts as for instance on the playground, in the classroom, or in the gymnasium; and evidence suggests that it increases with age. ${ }^{7}$

\section{NEUROMOTOR DISORDERS IN CHILDREN}

Cerebral palsy (CP) is one of the most common physical disabilities, which affects about 2-3 children per 1'000 live births in the Western countries. ${ }^{11}$ It is defined as "a group of permanent disorders in the development of movement and posture, causing activity limitations that are attributed to non-progressive disturbances that occurred in the developing fetal or infant brain. The motor disorders of CP are often accompanied by disturbances of sensation, perception, cognition, communication, and behavior, by epilepsy, and by secondary musculoskeletal problems".11 Although $\mathrm{CP}$ is a permanent disorder caused by a non-progressive lesion, its developmental consequences are often progressive due to the challenges posed by the environment, which become more and more demanding when growing up. ${ }^{12}$

The classification of $\mathrm{CP}$ is based on the dominant type of tonus or movement abnormality, categorized as spasticity, dystonia, choreoathetosis, or ataxia. Further, a differentiation of unilateral versus bilateral motor involvement is helpful. ${ }^{11}$

$\mathrm{CP}$ affects multiple dimensions of health. An extensive literature review showed high- and moderate-quality evidence that among children with $\mathrm{CP}$, three in four were in pain, one in two had an intellectual disability, one in three had a hip displacement, and one in three could not walk. Further, one in four could not talk, one in four had epilepsy, one in four had a behavior disorder, one in four had bladder control problems, one in five dribbled, one in ten was blind, one in 15 was tube-fed, and one in 25 was deaf. ${ }^{13}$ The experienced pain, fatigue, and impairments to body structure and function affect children's and youths' participation in school and leisure activities. ${ }^{14}$ Furthermore, young people with $\mathrm{CP}$ walk significantly less each day when compared to their typically developing peers and exhibit less habitual PA in daily life, regardless of their functional impairment level or their age. ${ }^{15,16} \mathrm{CP}$ is a 
lifelong condition, which can result in various secondary impairments or limitations, which impact on physical, social as well as mental well-being during the further course of life. ${ }^{17}$

The movement abilities of children with CP are characterized with the internationally widely employed expanded and revised Gross Motor Function Classification System (GMFCS - E\&R). ${ }^{18}$ The GMFCS - E\&R describes a child's current gross motor function based on its self-initiated movement with emphasis on sitting, walking, and wheeled mobility (Figure 1.1.). By grouping a child into one of five levels, using different age-bands, it describes what equipment or mobility devices a child needs to move at home, school, outdoors, or in the community. The described differentiations between consecutive levels represent differences in gross motor function that are supposed to be meaningful in children's everyday lives. With its properties, the GMFCS - E\&R facilitates the communication between health professionals and families regarding therapy goal setting, treatment planning, and prognosis concerning the expected level of independence. In clinical research, the GMFCS - E\&R can be used to classify children with CP into more homogeneous groups regarding their motor function. 


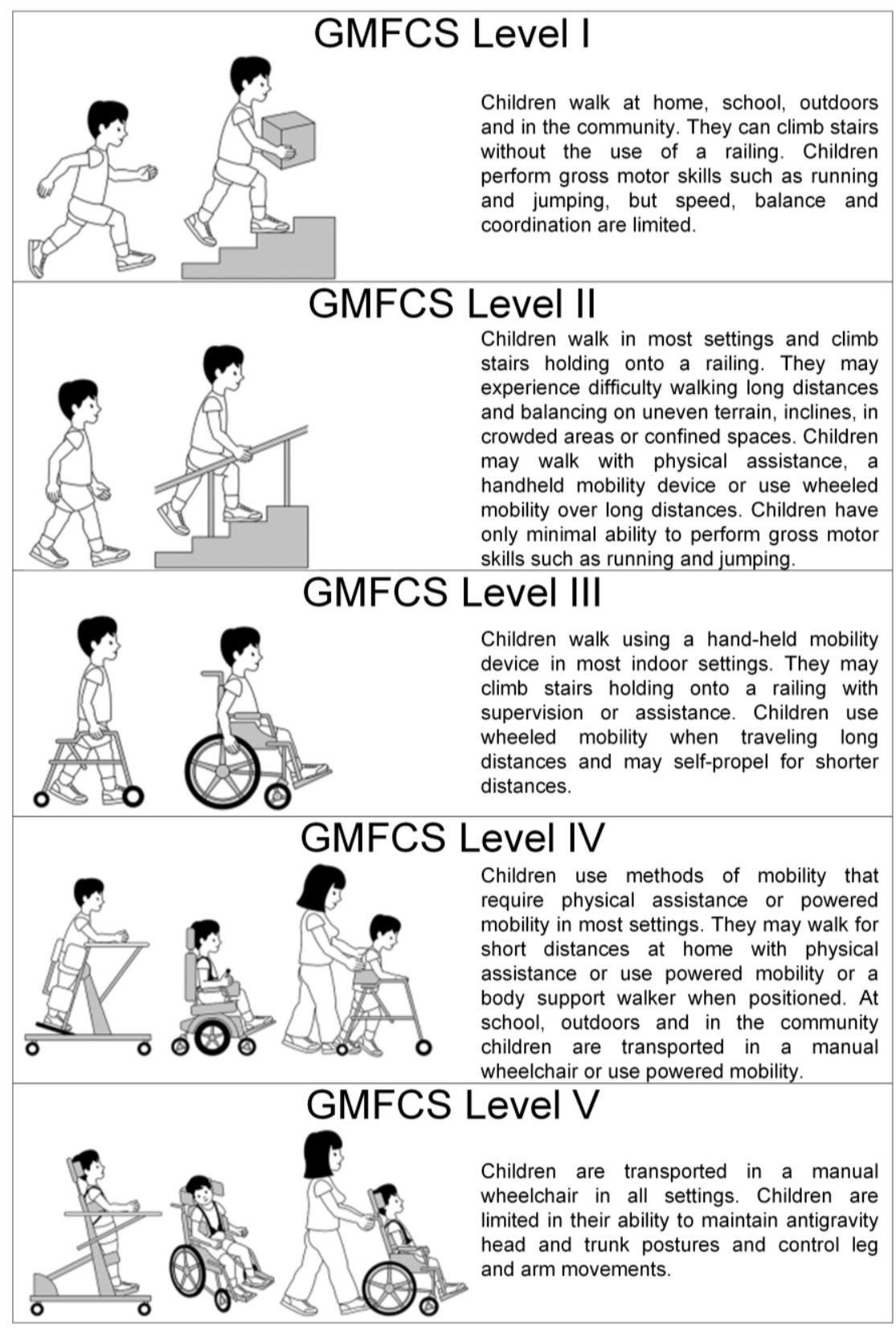

Figure 1.1. The Gross Motor Function Classification System - expanded and revised (GMFCS - E\&R) between 6th and 12th birthday: descriptors and illustrations.

The GMFCS - E\&R is a 5-level classification system that describes the gross motor function of children and youths with cerebral palsy based on their self-initiated movement with an emphasis on sitting, walking, and wheeled mobility.

GMFCS - E\&R descriptors: copyright (C) Palisano et al. $2008 .{ }^{18}$ Illustrations: copyright Version 2 () Bill Reid, Kate Willoughby, Adrienne Harvey and Kerr Graham, The Royal Children's Hospital Melbourne. 
However, it is essential to understand that as a classification tool, the GMFCS - E\&R was not designed to measure change over time, and seems therefore not to be the most appropriate choice as an outcome tool to measure change over time.

The described heterogeneity and multifaceted aspects of CP apply to many more neuromotor disorders in children, whether they are congenital, like CP, spina bifida, or genetic disorders - or acquired, like traumatic brain injury or stroke. Thus, most of these neuromotor disorders have a substantial impact on many different aspects of those children's lives.

\section{INTERNATIONAL CLASSIFICATION OF FUNCTIONING, DISABILITY AND HEALTH}

To describe and organize all health and health-related domains of functioning and disability in patients on individual and population levels, the World Health Organization (WHO) established in 2001 a theoretical framework and classification system - the International Classification of Functioning, Disability and Health - better known as the ICF.19 As developing children and youths have particular characteristics regarding health and functioning and are confronted with the influence of their environment, the ICF for Children and Youth (ICF-CY) was established in 2007. ${ }^{20}$ Like the ICF, the ICF-CY is structured on the one hand into the part "functioning and disability", which is further characterized by the components "body structures and functions" and "activities and participation", and on the other hand into the "contextual factors", which include the components "environmental factors" and "personal factors". These structures facilitate a holistic view when documenting or measuring a child's health and disability, as well as when planning or evaluating interventions in clinical and research settings. The ICF model illustrates the interaction of the included components (Figure 1.2.). 


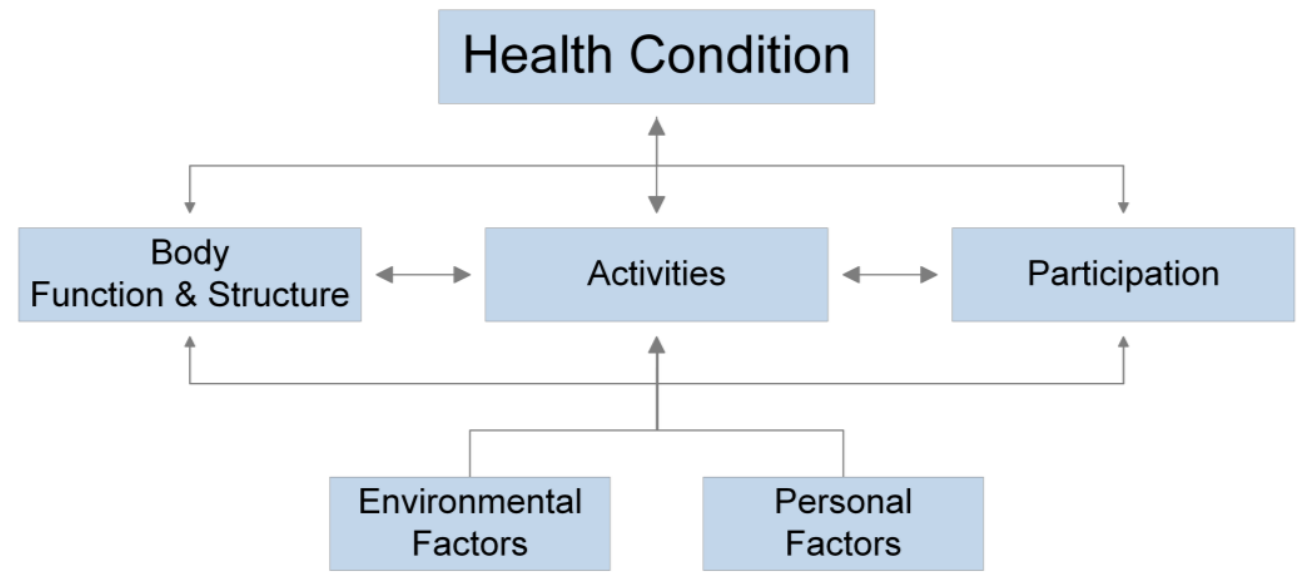

Figure 1.2. The International Classification of Functioning, Disability and Health (ICF) Model: Interaction between the ICF components.

The health condition of a person is viewed as result of a complex dynamic interaction of the different ICF components "body function", "body structure", "activities", and "participation" - with environmental and personal factors also influencing the person's functional status.

\section{PEDIATRIC NEUROREHABILITATION}

Associated with the heterogeneity of children's and youths' impairments, pediatric neurorehabilitation covers a vast area of different therapy concepts and intervention strategies that are supplied by health professionals from various specialties. Physical therapists, occupational therapists, speech and language therapists, and physicians work along with sports therapists, nurses, teachers, and (neuro-)psychologists in a multidisciplinary team on the overarching goal of pediatric neurorehabilitation, which is to enable children and youths with congenital or acquired neuromotor disorders to live their lives as independently as possible. While in past times, the priorities of rehabilitation predominantly lay on body structures and functions, the focus increasingly shifted to enhancing activities and participation within the last two decades. ${ }^{21}$ In other words, the goal is to find ways to enable children and their families to actively take part in society, despite the limitations that the disability imposes on them.

In a qualitative study, youths with $\mathrm{CP}$, parents, and medical professionals were asked which issues they found most important to consider when evaluating the effects of an intervention for children with CP. The answers to this open-ended query covered all ICF-CY components as well as concepts that are not included in the ICF- 
CY, like quality of life. All involved groups equally identified mobility as one domain of importance. ${ }^{22}$

There is a broad range of interventions that address gait or walking ability in children with neuromotor disorders. Conservative treatment approaches can be classified as either impairment-based or task-specific. ${ }^{23}$ Impairment-based approaches, for example strength training, focus on the treatment of the body structure and function component of the ICF-CY, while task-specific approaches, such as on-ground gait training or partial body-weight supported treadmill training (PBWSTT), target at the ICF-CY's activity and participation component.

Although a high number of studies have been performed on the effectiveness of gait training in children with neuromotor disorders, they are very heterogeneous in various aspects. Interventions expand from conventional overground gait training, ${ }^{24}$ home exercise programs, ${ }^{25}$ resistance training, ${ }^{26}$ biofeedback interventions ${ }^{27}$ and neurodevelopmental treatment ${ }^{28}$ to functional electrical stimulation, ${ }^{29}$ treadmill training with and without body-weight support ${ }^{30}$ to different types of robot-assisted gait training (RAGT). ${ }^{31}$ The implementation modes of these interventions vary from two to ten sessions per week, over a duration of two to twelve weeks, and patient samples include children with CP, Down syndrome, spinal cord injury, and mixed neurological diagnoses. ${ }^{23,30}$ Furthermore, most evidence originates from low-level methodological quality studies without control groups. ${ }^{23}$ Related to task-specific gait training, results of a recent meta-analysis showed a substantial effect of various interventions on gait speed in children with $\mathrm{CP} .{ }^{23}$ An earlier review on systematic reviews found promising results as well when looking at different kinds of treadmill training in pediatric neurorehabilitation, including treadmill training with or without partial body-weight support and RAGT. ${ }^{30}$

Nevertheless, there remains a significant lack of knowledge concerning the effectiveness as well as the optimal dosage of gait training in pediatric neurorehabilitation. This includes parameters like frequency, duration, number of training sessions, as well as information on the optimal intensity of the training regarding gait speed and percentage of body-weight support during PBWSTT or 
RAGT. Further, it remains unclear, whether children with different functional walking levels or diagnoses would profit from different types of gait training.

\section{ROBOT-ASSISTED GAIT TRAINING}

RAGT, being one of these various approaches of gait training, has become an increasingly common therapeutic intervention in pediatric neurorehabilitation over the last decade. Our rehabilitation center Affoltern am Albis of the University Children's Hospital Zurich, Switzerland was the first clinical center that implemented RAGT with the driven gait orthosis Lokomat ${ }^{\circledR}$ (Hocoma AG, Volketswil, Switzerland) in children and adolescents in 2005. The Lokomat automates gait therapy on a treadmill by two actuated leg orthoses, which are available in two different sizes (pediatric and adult modules). These can be individually adapted to the patient's legs and are attached with cuffs of various available sizes, while the patient is secured using a counter system with a harness providing partial body-weight support (Figure 1.3.). RAGT with the Lokomat has been shown to be a feasible and safe treatment option for children and youths. ${ }^{32,33}$ It facilitates a task-specific training with high levels of repetitions while the therapist can incorporate variations by changing the joints' range of motion, gait speed, amount of body-weight support, and guidance force, or by introducing additional tasks while the child walks on the treadmill. ${ }^{34}$ With these features, RAGT combines many characteristics that are important for motor learning. ${ }^{35}$ Moreover, RAGT with the Lokomat seems to induce physiological surface electromyography activity patterns when compared to overground walking, with activity patterns that are more physiological than when walking unsupported on a treadmill. 36,37

While children do not always feel excited to use robotic technology and may even be scared to a certain degree,$^{14}$ the modern technology appeals to parents, and also seems to raise high expectations regarding gains in their child's walking abilities and the development towards a more physiological gait pattern. ${ }^{38}$ Expectations that, however, have not yet been scientifically confirmed; although a few randomized controlled trials (RCTs) on the effectiveness of RAGT with the Lokomat in children with CP exist, they showed serious limitations in terms of study design, dropout rates, 
and lacking descriptions of the (control) interventions. ${ }^{39-41}$ Furthermore, these studies focused on body function measures. However, researchers who only use body function measures overlook the fact that children with a disability do not necessarily desire to walk in so-called typical gait patterns, but refer to their usual walking pattern as "normal". ${ }^{2} \mathrm{~A}$ recent systematic review analyzed the evidence on the optimal application mode and the effectiveness of RAGT in children with various gait disorders. ${ }^{31}$ The authors concluded that clear training prescriptions and delivery modes for RAGT in children are absent and that evidence of its effectiveness is still weak and inconsistent.

Another factor that hampers the gain of evidence regarding the effectiveness of RAGT in children is the huge variety of outcome measures that have been used in the studies so far. ${ }^{30,31}$ Thus, to increase the impact of single studies and herewith the overall evidence in this field, higher-level trial designs are needed to evaluate the effectiveness of RAGT in children across different diagnoses, ages, and outcome domains. Additionally, it is crucial to apply a more consistent assessment approach across studies, including measures that are relevant for the children and their families within the different components of the ICF-CY. ${ }^{43}$ For the time being, while waiting for new high-quality evidence, clinicians and researchers can find advice within the practical recommendations of an international expert panel on how to apply RAGT in children with CP. ${ }^{34}$ 


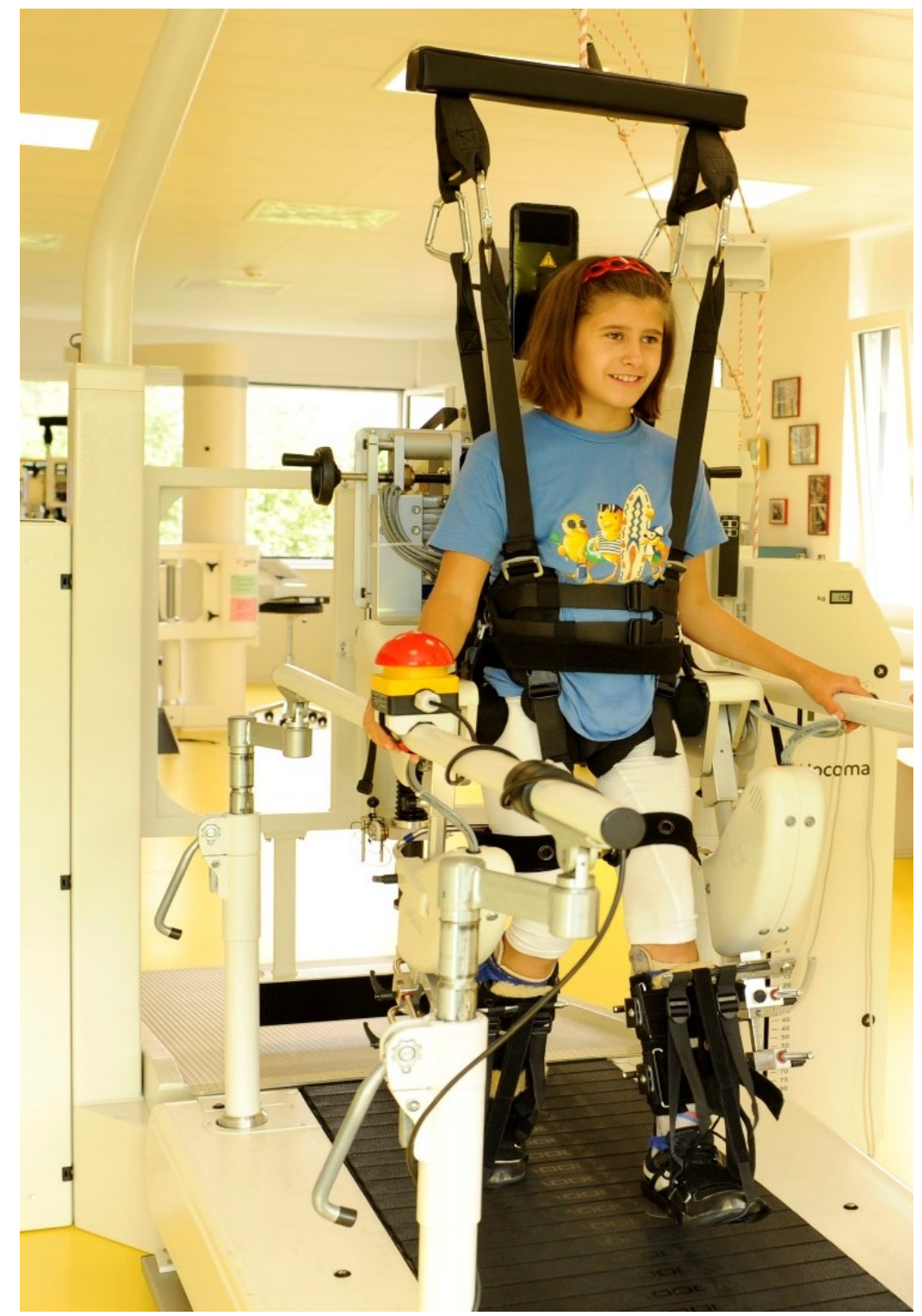

Figure 1.3. Robot-assisted gait training (RAGT) of a child on the Lokomat (Hocoma, Volketswil, Switzerland).

The two actuated leg orthoses are individually adapted to the patient's legs. The exoskeleton's movements are controlled and adjusted by the Lokomat software, which allows the regulation of kneeand hip-range of motion, treadmill speed, and the supportive force of the drives. 


\section{MEASUREMENT INSTRUMENTS ASSESSING WALKING ABILITY}

Measures that evaluate the effect of RAGT address mainly gait function, which can be linked to the components "body structures and functions" as well as "activities and participation" of the ICF-CY structure. Activity and participation measures can be further differentiated into the constructs "capacity" and "performance" (Figure 1.4.). ${ }^{20}$

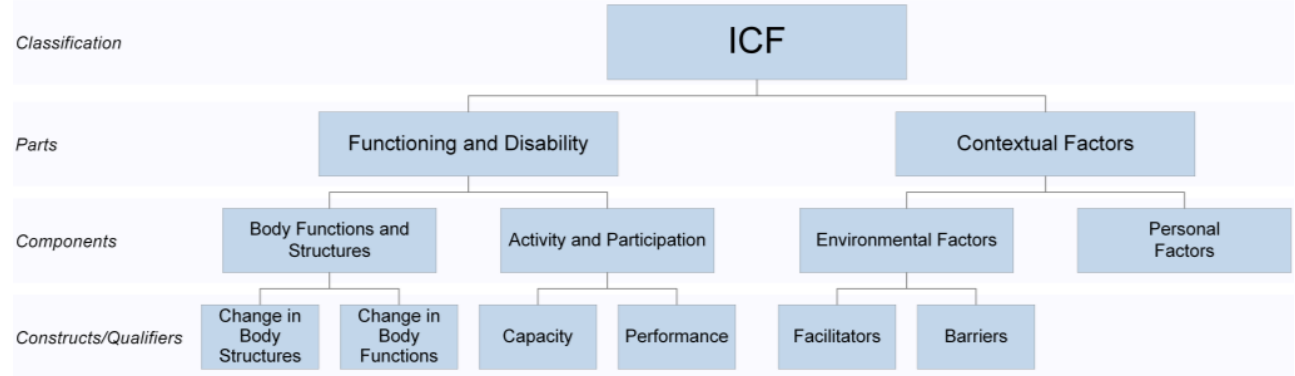

Figure 1.4. The International Classification of Functioning, Disability and Health (ICF) structure.

The two constructs "performance" and "capacity" can be used to indicate how the environment influences a person's activities and participation, and how environmental change may improve a person's functioning.

While capacity describes what a child "can do in a standardized, controlled environment," performance refers to what a child "actually does do in his or her daily environment." A third construct, which is not part of the ICF-CY structure, is "capability", which describes what a child "can do in his or her daily environment." Thus, the impact of the environment is minimized as much as possible when assessing capacity, whereas the physical environment is taken into account for the assessment of a child's capability, and both, the physical and social environment, are considered when evaluating a child's performance. Consequently, a discriminating factor between these three constructs is the interaction between a person and the environment (Figure 1.5.).

In real life, we are always exposed to the influence of the social and physical environment. Given the significance of enabling children with a disability and their families to live an as independent life as possible, gait interventions and definitions of goal attainment should therefore reflect activities or participation in the scope of performance. "The gap between capacity and performance reflects the difference 
between the impacts of current and uniform environments, and thus provides a useful guide as to what can be done to the environment of the individual to improve performance. $" 9$

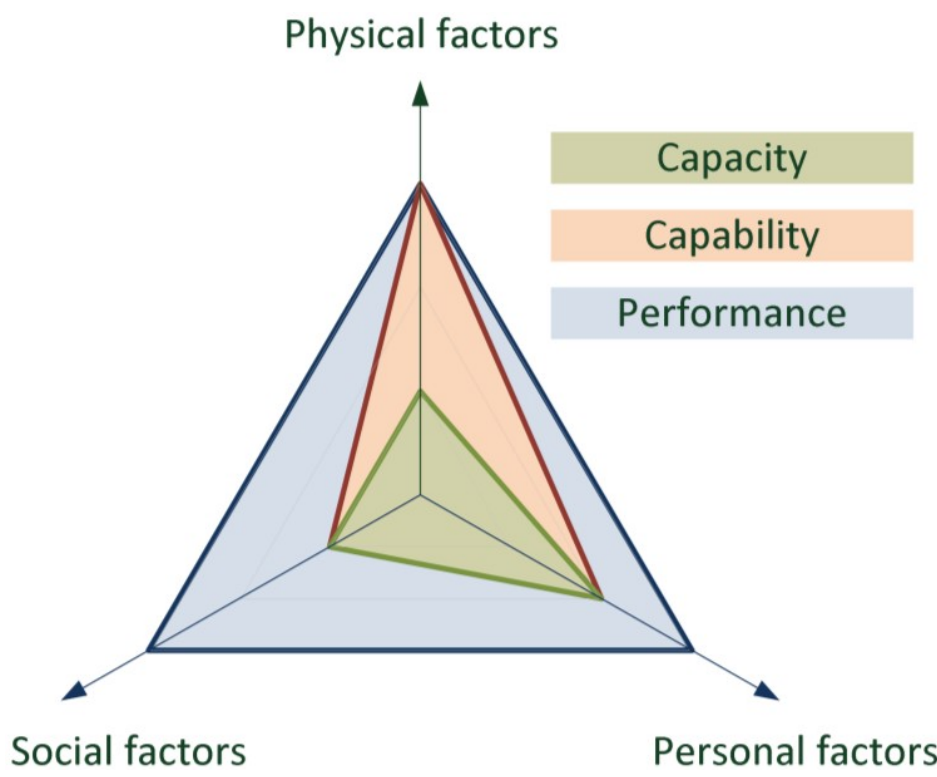

Figure 1.5. Impact of various factors on the constructs "capacity," "capability," and "performance." The three triangles schematically illustrate the influence of the different factors on the constructs "capacity", "capability", and "performance"; the further away the corner points are from the center of the axes, the larger is the impact of this factor on the construct.

Nevertheless, in practice, the primary focus of studies in children with CP lies on assessing the functioning and disability part of the ICF-CY, while the contextual factors are far less frequently examined. ${ }^{43}$ Frequently applied measures to document changes after gait interventions in children are manual muscle tests for the assessment of muscle strength and range of motion measurements to evaluate joint mobility. Besides these measures of body function, also activity measures reflecting capacity are widely used, such as the Gross Motor Function Measure (GMFM), which may be considered as the criterion measure of gross motor function in children with $\mathrm{CP}$. Also popular are timed walking tests, for example the 6-minute walk test, for which children have to cover as many meters as possible within six minutes, or 10-meter walk tests, which measure the time a child takes to walk ten meters at their preferred or maximal speed. Performance measures, however, are only rarely used 
in studies which evaluate the effectiveness of different kinds of treadmill training in children with neuromotor disorders. ${ }^{30}$

Another area of importance when choosing appropriate measures besides the above-mentioned ICF-CY components is to consider the measurement goals. Depending on whether the objective of the measurement is prognostic, diagnostic, or evaluative, different psychometric properties are given priority in the patient population in question. To assess the effectiveness of an intervention, validity (the degree to which an instrument measures the construct(s) it purports to measure), reliability (the extent to which scores for patients who have not changed are the same for repeated measurements under several conditions) and especially responsiveness (the ability of an instrument to detect change over time in the construct to be measured) are crucial properties of a test instrument. ${ }^{45}$ However, evidence so far showed that although many different measures exist to assess various activities of daily living ( $A D L)$ or activity limitations in children and adolescents with neuromotor disorders, most of these tools require further research to gain full clarity on their psychometric properties. ${ }^{46-49}$

\section{CLINICAL BACKGROUND, AIMS, AND OUTLINE OF THIS PHD-THESIS}

RAGT with the Lokomat was implemented in the neuropediatric treatment regime of our rehabilitation center more than ten years ago, but evidence of its effectiveness in the pediatric population is still mainly restricted to case series studies and inconclusive (randomized) controlled trials. ${ }^{32,39-41,50-55}$ Also, our practical experience is ambiguous: RAGT with the Lokomat is only one piece of the puzzle of various interventions within a multi-modal approach during inpatient rehabilitation in our center that affects gait function. Thus, improvements in gait function after an inpatient rehabilitation period cannot solely be attributed to the Lokomat training. Furthermore, parents often report an improvement in their child's gait function after an outpatient RAGT period, which we cannot necessarily quantify with the outcome measures that are included in our standardized assessment procedure.

Our recurring observation that subjectively perceived improvement cannot be objectified with our applied measurement instruments made us realize and 
appreciate the significant role of outcome assessment in clinical practice as well as in research. As pediatric intervention studies often lack large sample sizes, the gain of evidence depends not only on high-quality trials but also on the possibility of pooling the results of comparable studies. It requires, on the one hand, the availability of psychometrically sound and clinically relevant and applicable outcome instruments, and on the other hand a harmonization of the applied outcome tools while still ensuring coverage of all ICF-CY components.

Consequently, we aimed to summarize the evidence of gait instruments and their psychometric properties in neuropediatric populations within a systematic review. We present the results of this systematic review on gait-related outcomes in children with neuromotor disorders in chapter 2. Based on the systematic review, we aimed to select outcome measures reflecting ICF-CY components and constructs that are so far un- or underrepresented in our clinic and that we considered relevant and feasible to be applied in our pediatric inpatient neurorehabilitation setting. With the Functional Mobility Scale (FMS) and the Gillette Functional Assessment Questionnaire - walking scale ( $F A Q)$, we chose two mobility measures that reflect the component "activity" and the construct "performance" within the ICF-CY. We translated these measures into German and report on their validity (chapter 3), reliability (chapter 4), and responsiveness and minimal important change (chapter 5) in our inpatient neurorehabilitation population.

A further aim of this PhD-project was to evaluate the effectiveness of RAGT with the Lokomat in children and adolescents with CP. Pediatric Lokomat training involves a considerable amount of resources - from the patient and family side (time, costs, travel expenses as pediatric Lokomats are available at only two locations in all of Switzerland), the health care providers (infrastructure, training of staff, number of therapists per training), and health insurance companies (meeting the costs). Considering all these resources, a clarification of its effectiveness in our pediatric population is paramount. Our RCT already started in 2009, and as we wanted to incorporate the circumstances and conditions of the everyday clinical practice as much as possible, we chose a pragmatic trial design. This design also reflects the 
outcome measures that we have used and still use today as standard to measure the therapeutic progress of children performing RAGT in our clinic.

We present the detailed study protocol of this pragmatic RCT on the effectiveness of RAGT in chapter 6 and discuss the results of the recently completed trial in chapter 7. In chapter 8 , we provide an overall discussion about the findings of this PhDthesis.

\section{REFERENCES}

1. http://medical-dictionary.thefreedictionary.com/Mobility.

2. Biringen Z, Emde R, Campos J, Appelbaum M. Affective reorganization in the infant, the mother, and the dyad: the role of upright locomotion and its timing. Child Dev. 1995;66(2):499-514.

3. Campos JJ, Anderson DI, Barbu-Roth M, Hubbard EM, Hertenstein MJ, Witherington D. Travel Broadens the Mind. Infancy. 2000;1(2):149-219.

4. Clearfield MW. Learning to walk changes infants' social interactions. Infant Behav Dev. 2011;34(1):15-25.

5. Walle EA, Campos JJ. Infant language development is related to acquisition of walking. Dev Pschology. 2014;50(2):336-348.

6. Leonard HC, Hill EL. Review: The impact of motor development on typical and atypical social cognition and language: A systematic review. Child Adolesc Ment Health. 2014;19(3):163-170.

7. Logan SW, Ross SM, Schreiber MA, et al. Why We Move: Social Mobility Behaviors of Non-Disabled and Disabled Children across Childcare Contexts. Front Public Heal. 2016;4(September).

8. Marin L, Weise I, Adolph KE. Locomotor Development. In: Balter L, ed. Parenthood in America: An Encyclopedia. Denver; 2000:354-358.

9. Bjornson K, Belza B, Kartin D, R L, McLaughlin J. Ambulatory physical activity performance in youth with cerebral palsy and youth who are developing typically. Phys Ther. 2007;87(3):248-257.

10. van den Berg-Emons R, Saris W, de Barbanson D, Westerterp K, Huson A, van Baak M. Daily physical activity of schoolchildren with spastic diplegia and of healthy control subjects. J Pediatr. 1995;127(4):578-584.

11. Rosenbaum $P$, Paneth $N$, Leviton $A$, et al. A report: The definition and classification of cerebral palsy April 2006. Dev Med Child Neurol. 2007;49(SUPPL. 109):8-14. 
12. Christine $\mathrm{C}$, Dolk $\mathrm{H}$, Platt $\mathrm{M}$, et al. Recommendations from the SCPE collaborative group for defining and classifying cerebral palsy. Dev Med Child Neurol Suppl. 2007;Feb(109):35-38.

13. Novak I, Hines M, Goldsmith S, Barclay R. Clinical prognostic messages from a systematic review on cerebral palsy. Pediatrics. 2012;130(5):e1285-312.

14. Lindsay S. Child and youth experiences and perspectives of cerebral palsy: a qualitative systematic review. Child Care Health Dev. 2016;42(2):153-175. doi:10.1111/cch.12309.

15. Bjornson KF, Zhou C, Stevenson R, Christakis D, Song K. Walking activity patterns in youth with cerebral palsy and youth developing typically. Disabil Rehabil. 2014;36(15):1279-1284.

16. Carlon SL, Taylor NF, Dodd KJ, Shields N. Differences in habitual physical activity levels of young people with cerebral palsy and their typically developing peers: a systematic review. Disabil Rehabil. 2013;35(8):647-655.

17. Gajdosik CG, Cicirello N. Secondary conditions of the musculoskeletal system in adolescents and adults with cerebral palsy. Phys Occup Ther Pediatr. 2001;21(4):49-68.

18. Palisano RJ, Rosenbaum $P$, Bartlett $D$, Livingston $M H$. Content validity of the expanded and revised Gross Motor Function Classification System. Dev Med Child Neurol. 2008;50(10):744-750.

19. World Health Organisation. International Classification of Functioning, Disability and Health (ICF). Geneva: World Health Organization; 2001.

20. World Health Organisation. International Classification of Functioning, Disability and Health: Children \& Youth Version: ICF-CY. Geneva: World Health Organization; 2007.

21. Bjornson KF, Zhou C, Stevenson RD, Christakis D. Relation of stride activity and participation in mobility-based life habits among children with cerebral palsy. Arch Phys Med Rehabil. 2014;95(2):360-368.

22. Vargus-Adams JN, Martin LK. Domains of importance for parents, medical professionals and youth with cerebral palsy considering treatment outcomes. Child Care Health Dev. 2011;37(2):276-281.

23. Moreau NG, Winter AB, Bjornson K, Hobbs A, Soileau M, Lahasky K. Effectiveness of Rehabilitation Interventions to Improve Gait Speed in Children With Cerebral Palsy: Systematic Review and Meta-analysis. Phys Ther. 2016;96(12):1938-1954.

24. Swe NN, Sendhilnnathan S, van Den Berg M, Barr C. Over ground walking and body weight supported walking improve mobility equally in cerebral palsy: a randomised controlled trial. Clin Rehabil. 2015;29(11):1108-1116. 
25. Paleg G, Livingstone R. Outcomes of gait trainer use in home and school settings for children with motor impairments: a systematic review. Clin Rehabil. 2015;29(11):1077-1091.

26. Lee J, Sung I, Yoo J. Therapeutic effects of strengthening exercise on gait function of cerebral palsy. Disabil Rehabil. 2008;30(19):1439-1444.

27. Dursun E, Dursun N, Alican D. Effects of biofeedback treatment on gait in children with cerebral palsy. Disabil Rehabil. 2004;26(2):116-120.

28. Desloovere K, De Cat J, Molenaers G, et al. The effect of different physiotherapy interventions in post-BTX-A treatment of children with cerebral palsy. Eur $\mathrm{J}$ Paediatr Neurol. 2012;16(1):20-28.

29. Moll I, Vles J, Soudant D, et al. Functional electrical stimulation of the ankle dorsiflexors during walking in spastic cerebral palsy: a systematic review. Dev Med Child Neurol. 2017;59(12):1230-1236.

30. Zwicker JG, Mayson TA. Effectiveness of Treadmill Training in Children With Motor Impairments : An Overview. Pediatr Phys Ther. 2010;22:361-377.

31. Lefmann S, Russo R, Hillier S. The effectiveness of robotic-assisted gait training for paediatric gait disorders: systematic review. J Neuroeng Rehabil. 2017;14(1):1.

32. Meyer-Heim A, Borggraefe I, Ammann-Reiffer C, et al. Feasibility of roboticassisted locomotor training in children with central gait impairment. Dev Med Child Neurol. 2007;49:900-906.

33. Borggraefe I, Klaiber M, Schuler T, et al. Safety of robotic-assisted treadmill therapy in children and adolescents with gait impairment: a bi-centre survey. Dev Neurorehabil. 2010;13(2):114-119.

34. Aurich T, Warken B, Graser J V., et al. Practical Recommendations for RobotAssisted Treadmill Therapy (Lokomat) in Children with Cerebral Palsy: Indications, Goal Setting, and Clinical Implementation within the WHO-ICF Framework. Neuropediatrics. 2015;46(4):248-260.

35. Cano-de-la-Cuerda R, Molero-Sánchez a, Carratalá-Tejada M, et al. Theories and control models and motor learning: clinical applications in neuro-rehabilitation. Neurologia. 2015;30(1):32-41.

36. Aurich Schuler T, Müller R, van Hedel HJA. Leg surface electromyography patterns in children with neuro-orthopedic disorders walking on a treadmill unassisted and assisted by a robot with and without encouragement. $J$ Neuroeng Rehabil. 2013;10:78.

37. Aurich-Schuler $T$, Grob $F$, van Hedel $H$, Labruyère R. Can Lokomat therapy with children and adolescents be improved? An adaptive clinical pilot trial comparing Guidance force, Path control, and FreeD. J Neuroeng Rehabil. 2017;14:76. 
38. Beveridge B, Feltracco D, Struyf J, et al. "You gotta try it all": Parents' Experiences with Robotic Gait Training for their Children with Cerebral Palsy. Phys Occup Ther Pediatr. 2015;35(4):327-341.

39. Wallard L, Dietrich G, Kerlirzin Y, Bredin J. Effect of robotic-assisted gait rehabilitation on dynamic equilibrium control in the gait of children with cerebral palsy. Gait Posture. 2017;11(60):55-60.

40. Wallard L, Dietrich G, Kerlirzin Y, Bredin J. Robotic-assisted gait training improves walking abilities in diplegic children with cerebral palsy. Eur J Paediatr Neurol. 2017;21(3):557-564.

41. Druzbicki M, Rusek W, Snela $S$, et al. Functional effects of robotic-assisted locomotor treadmill therapy in children with cerebral palsy. J Rehabil Med. 2013;45(4):358-363.

42. Phelan SK, Gibson BE, Wright FV. What is it like to walk with the help of a robot? Children's perspectives on robotic gait training technology. Disabil Rehabil. 2015;8288(November):1-10.

43. Schiariti V, Klassen AF, Cieza A, et al. Comparing contents of outcome measures in cerebral palsy using the international classification of functioning (ICF-CY): A systematic review. Eur J Paediatr Neurol. 2014;18(1):1-12.

44. Holsbeeke L, Ketelaar M, Schoemaker MM, Gorter JW. Capacity, capability, and performance: different constructs or three of a kind? Arch Phys Med Rehabil. 2009;90(5):849-855.

45. Mokkink LB, Terwee CB, Patrick DL, et al. The COSMIN study reached international consensus on taxonomy, terminology, and definitions of measurement properties for health-related patient-reported outcomes. J Clin Epidemiol. 2010;63(7):737-745.

46. Debuse D, Brace H. Outcome measures of activity for children with cerebral palsy: a systematic review. Pediatr Phys Ther. 2011;23(3):221-231.

47. Harvey A, Robin J, Morris ME, Graham HK, Baker R. A systematic review of measures of activity limitation for children with cerebral palsy. Dev Med Child Neurol. 2008;50(3):190-198.

48. Adair B, Said CM, Rodda J, Morris ME. Psychometric properties of functional mobility tools in hereditary spastic paraplegia and other childhood neurological conditions. Dev Med Child Neurol. 2012;54(7):596-605.

49. Sakzewski L, Boyd R, Ziviani J. Clinimetric properties of participation measures for 5- to 13-year-old children with cerebral palsy: a systematic review. Dev Med Child Neurol. 2007;49(3):232-240.

50. Borggraefe I, Schaefer JS, Klaiber M, et al. Robotic-assisted treadmill therapy improves walking and standing performance in children and adolescents with cerebral palsy. Eur J Paediatr Neurol. 2010;14(6):496-502. 
51. Meyer-Heim A, Ammann-Reiffer C, Schmartz A, et al. Improvement of walking abilities after robotic-assisted locomotion training in children with CP. Arch Dis Child. 2009;94(8):615-620.

52. Smania N, Bonetti P, Gandolfi M, et al. Improved gait after repetitive locomotor training in children with cerebral palsy. Am J Phys Med Rehabil. 2011;90(2):137149.

53. Borggraefe I, Meyer-Heim A, Kumar A, Schaefer J, Berweck S, Heinen F. Improved gait parameters after robotic-assisted locomotor treadmill therapy in a 6year-old child with cerebral palsy. Mov Disord. 2008;23(2):280-283.

54. Schroeder A, von Kries R, Riedel C, et al. Patient-specific determinants of responsiveness to robot-enhanced treadmill therapy in children and adolescents with cerebral palsy. Dev Med Child Neurol. 2014;56(12):1172-1179.

55. Schroeder A, Homburg M, Warken B, et al. Prospective controlled cohort study to evaluate changes of function, activity and participation in patients with bilateral spastic cerebral palsy after Robot-enhanced repetitive treadmill therapy. Eur $J$ Paediatr Neurol. 2014;18(4):502-510. 
CHAPTER 2

\section{MEASUREMENT PROPERTIES OF GAIT- RELATED OUTCOMES IN YOUTH WITH NEUROMUSCULAR DIAGNOSES: A SYSTEMATIC REVIEW}

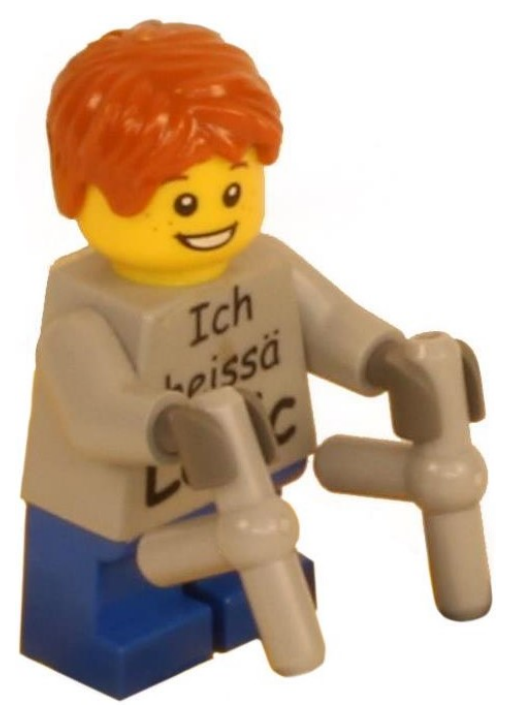

This chapter has been published as:

Ammann-Reiffer C, Bastiaenen CHG, de Bie RA, van Hedel HJA. Measurement properties of gait-related outcomes in youth with neuromuscular diagnoses: a systematic review. Phys Ther. 2014;94(8):1067-82. 


\section{ABSTRACT}

BACKGROUND: Sound measurement properties of outcome tools are essential when evaluating outcomes of an intervention, in clinical practice and in research.

PURPOSE: The purpose of this study was to review the evidence on reliability, measurement error, and responsiveness of measures of gait function in children with neuromuscular diagnoses.

DATA SOURCES: The MEDLINE, CINAHL, EMBASE, and PsycINFO databases were searched up to June 15, 2012.

STUDY SELECTION: Studies evaluating reliability, measurement error, or responsiveness of measures of gait function in 1- to 18-year-old children and youth with neuromuscular diagnoses were included.

DATA EXTRACTION: Quality of the studies was independently rated by two raters using a modified COnsensus-based Standards for the selection of health status Measurement INstruments (COSMIN) checklist. Studies with a fair quality rating or better were considered for best evidence synthesis.

DATA SYNTHESIS: Regarding the methodological quality, 32 out of 35 reliability studies, all of the 13 measurement error studies, and five out of 10 responsiveness studies were of fair or good quality. Best evidence synthesis revealed moderate to strong evidence for reliability for several measures in children and youth with cerebral palsy (CP) but was limited or unknown in other diagnoses. The Functional Mobility Scale (FMS) and the Gross Motor Function Measure (GMFM) dimension E showed limited positive evidence for responsiveness in children with $\mathrm{CP}$, but it was unknown or controversial in other diagnoses. No information was reported on the minimal important change; thus, evidence on measurement error remained undetermined.

LIMITATIONS: As studies on validity were not included in the review, a comprehensive appraisal of the best available gait-related outcome measure per diagnosis is not possible. 
CONCLUSIONS: There is moderate to strong evidence on reliability for several measures of gait function in children and youth with $C P$, whereas evidence on responsiveness exists only for the FMS and the GMFM dimension E. 


\section{INTRODUCTION}

Achieving, restoring, or sustaining the ability to walk is one of the main goals when rehabilitating children with congenital or acquired neuromuscular diagnoses. Although one in two children with cerebral palsy (CP) can walk independently, one in six use walking aids and thus are at high risk to lose walking function during adolescence. ${ }^{1}$

To evaluate interventions and monitor progress, valid, reliable, and responsive assessment tools are essential. ${ }^{2}$ Information on validity (the degree to which an instruments measures the construct it purports to measure), reliability (the degree to which the measurement is free from measurement error), and responsiveness (the ability of an instrument to detect change over time in the construct to be measured) is a prerequisite in the decision-making process of determining which measures should be chosen in the clinical setting as well as in research.

The International Classification of Functioning, Disability and Health (ICF) of the World Health Organization provides a theoretical framework and classification system for describing and measuring health and health-related states. ${ }^{3}$ In 2007, the ICF was complemented for children and youth (ICF-CY). ${ }^{4}$ Like the ICF, the ICF-CY classifies outcome into four domains: "body function and structure", "activity" (the execution of a task or action by an individual), "participation" (the person's involvement in a life situation), and "environmental and personal factors." When monitoring progress, it is important to distinguish between what a patient is capable of in a standardized controlled environment (capacity) and what the patient actually does in his or her daily environment (performance). ${ }^{5}$

Interventions are often carried out with the goal of affecting clinically important activity and participation domains. Bjornson et al., for example, demonstrated this approach in their comprehensive evaluation of the effect of Botulinum Toxin, not only on spasticity but also on functional activities in children with CP. ${ }^{6}$ Therefore, the evaluation of measures being classified in these domains is needed.

Systematic reviews on measurement properties of assessment tools can facilitate the clinician's or researcher's search for an appropriate measurement instrument. ${ }^{7}$ Knowledge on the reliability, measurement error, and responsiveness of evaluative 
measurement tools, in particular, is important for the evaluation and clinical interpretation of change scores. In quantifying gait and gait-related outcome, some systematic reviews focused on activity and participation measures in children with $\mathrm{CP},{ }^{8-12}$ dynamic balance in children with acquired brain injury, ${ }^{13}$ functional mobility tools in children with hereditary spastic paraplegia and other childhood neurological problems,${ }^{14}$ or computerized gait analysis techniques in children with CP or spina bifida. ${ }^{15}$ Young and Wright identified physical function scales appropriate for pediatric orthopaedics, ${ }^{16}$ and a recent review evaluated the 6-Minute Walk Test (6MinWT) in children with chronic conditions. ${ }^{17}$ Children especially not only use gait to get from place to place but also integrate gait into all kinds of playful activities. Consequently, different constructs have to be considered when assessing gait function in children. To our knowledge, no review has summarized the evidence on the different constructs that are an integral part of gait function, nor do we know of any review that included studies on the whole spectrum of existing neuromuscular diagnoses in children. Therefore, the purposes of this literature review were: (1) to give an overview of available capacity and performance measures for the evaluation of gait function in children and youth with neuromuscular diagnoses and (2) to evaluate the current level of evidence for reliability, measurement error, and responsiveness of those measures.

\section{METHODS}

\section{Data sources and searches}

We performed an electronic search on June 15, 2012, in MEDLINE (via PubMed 1966-2012), CINAHL (via EBSCO 1981-2012), EMBASE (via embase.com 19742012), and PsycINFO (via EBSCO 1806-2012). Key search terms and MeSH terms were separately searched in three main filters, which comprised the construct (gait function), the target population (child AND neuromuscular diagnosis), and the measurement properties, and were then combined with an exclusion filter. In PubMed, a validated search filter for finding studies on measurement properties was used. ${ }^{18}$ The full search strategy is described in supplemental Table 2.2. We also reviewed the references of the included articles to identify additional eligible articles. 
Additionally, a second search was performed in PubMed, including the identified outcome instruments in combination with the terms for the target population.

\section{Study selection}

Screening of titles and abstracts as well as reviewing potentially eligible full-text articles were independently performed by two reviewers (C.A. and H.vH.). Cases of disagreement were discussed until consensus was reached. To be included, studies had to meet the following a priori formulated criteria: (1) the study evaluated capacity or performance measures related to gait function, thus being classified as activity or participation measure according to the ICF model; (2) constructs included were walking ability, functional mobility, speed, endurance and dynamic balance, and all constructs had to be measured during walking or running, or they were excluded (e.g., cardiorespiratory fitness during bicycle ergonometry); (3) the study population consisted of 1- to 18-year-old children and youth with neuromuscular diagnoses or developmental disabilities; (4) the study aim was the evaluation of reliability, measurement error, or responsiveness of the measure under question; and (5) articles were published in English or German as original articles in peer-reviewed journals. Semistructured interviews, multi-item scales with less than $50 \%$ of the items being gait specific, and individualized tools not exclusively focusing on gait were excluded, as well as articles containing translations or transcultural adaptations of measures into languages other than English, German, or Dutch. Falls and static balance were not considered gait specific. Anaerobic and aerobic measures of pulmonary function, even when tested during walking, were considered body functions in the ICF model; thus, papers dealing with only these constructs were also excluded.

\section{Data extraction and quality assessment}

We used a standardized protocol. Because no software was available, we developed a database based on Microsoft Access 2010 (Microsoft Corp, Redmond, Washington) for comprehensive data management, including paper selection, methodological quality scoring, and the data extraction process.

Evaluation of the methodological quality of the included studies 
Two reviewers (C.A. and $\mathrm{H} . \mathrm{vH}$.) independently evaluated the methodological quality of the included studies using the 4-point rating scale of the COnsensus-based Standards for the selection of health status Measurement INstruments (COSMIN) checklist. This instrument was developed in an international, multidisciplinary Delphi study to evaluate the methodological quality of studies on measurement properties of health status instruments. ${ }^{19-21}$ Within the COSMIN checklist, we used the three boxes evaluating the methodological quality of the studies on reliability, measurement error, and responsiveness. Whereas reliability is defined as the proportion of the total variance in the measurements which is due to "true" differences among patients, measurement error stands for the systematic and random error of a patient's score that is not attributed to true changes in the construct to be measured. ${ }^{22}$ Responsiveness is defined as the ability to detect change over time in the construct to be measured. ${ }^{22}$ Each item within a box was rated as "excellent", "good", "fair," or "poor." An overall score for the methodological quality of the study was determined by taking the lowest rating of any of the items in a box. Cases of disagreement were discussed until consensus was reached or, where not possible, resolved by a third, independent reviewer (C.B.). In every COSMIN box, there is an item concerning the sample size requirements. For a "fair" rating of this item, a study has to have at least 30 participants. In the neuropediatric field, this criterion would lead to a "poor" rating for many of the psychometric studies that otherwise would be scored at least as "fair". As this criterion would result in a loss of these articles for best evidence synthesis, we decided to use a modified COSMIN checklist and omit the sample size item from the quality assessment. Instead, in line with previous systematic reviews and after consultation with authors from the COSMIN checklist, we accounted for it at the best evidence synthesis stage. ${ }^{17,23}$

Data extraction

General characteristics of the instruments and data on interpretability and generalizability of the study results were extracted using the two corresponding COSMIN checklist boxes. A part of the CanChild Outcome Measures Rating Form was completed to describe the clinical utility of the evaluated outcome measures in terms of test format, time for test administration, required assessor training, and 
costs (supplemental Table 2.1.). ${ }^{24}$ If essential information was missing in the original publication, the authors were contacted to provide these data.

Best evidence synthesis

Based on quality criteria proposed by Terwee et al., ${ }^{25}$ one reviewer (C.A.) rated the results of the measurement properties for each study as positive, indeterminate, or negative (Tab. 2.1.). If studies evaluating the same outcome measure were sufficiently homogenous concerning the study population, design, and measurement procedure, an overall rating, adjusted for methodological quality, was performed. The best evidence synthesis included only results from studies rated as being of "excellent," "good," or "fair" methodological quality on the COSMIN. The level of overall evidence was rated on the basis of the strategy from the Cochrane Back Review Group as "strong," "moderate," "limited," "conflicting," or "unknown" (Tab. 2.2.). ${ }^{26}$

To account for the sample size, the level of evidence was rated as "strong", when the total sample size of combined studies was $\geq 100$, "moderate" for a total sample size between 50 and 99, "limited" for a total sample size between 25 and 49, and "unknown" when the sample size was fewer than $25 .{ }^{23}$ 
Table 2.1. Quality criteria for measurement properties

\begin{tabular}{|l|c|l|}
\hline \multicolumn{1}{|c|}{ Property } & Rating & \multicolumn{1}{c|}{ Quality criteria } \\
\hline Reliability & + & ICC/weighted kappa $\geq .70$ OR Pearson $r \geq .80$ \\
\hline & $?$ & Neither ICC/weighted kappa nor Pearson $r$ determined \\
\hline Measurement error & + & ICC/weighted kappa $<.70$ OR Pearson $r<.80$ \\
\hline & $?$ & MIC $>$ SDC OR MIC outside the LOA \\
\hline Responsiveness & - & MIC $\leq$ SDC OR MIC equals or inside LOA \\
\hline & + & Correlation with an instrument measuring the same construct $\geq .50$ \\
\hline & $?$ & $\begin{array}{l}\text { OR at least 75\% of the results are in accordance with the } \\
\text { hypotheses }\end{array}$ \\
\hline & - & $\begin{array}{l}\text { Solely correlations determined with unrelated constructs } \\
\text { than with unrelated constructs }\end{array}$ \\
\hline & & OR $<75 \%$ of the results are in accordance with the hypotheses \\
\hline & & $\begin{array}{l}\text { OR AUC }<0.70 \text { OR correlation with related constructs is lower than } \\
\text { with unrelated constructs }\end{array}$ \\
\hline
\end{tabular}

$a_{+}=$positive results, $?=$ indeterminate results, $-=$ negative results, $\mathrm{ICC}=$ intraclass correlation coefficient, $\mathrm{MIC}=$ minimal important change, $\mathrm{SDC}=$ smallest detectable change, LoA=limits of agreement, $\mathrm{AUC}=$ area under the receiver operating characteristic curve. Adapted with permission of Elsevier from: Terwee CB, Bot SD, de Boer MR, et al. Quality criteria were proposed for measurement properties of health status questionnaires. J Clin Epidemiol. 2007;60:34-42.

Table 2.2. Levels of evidence for the overall quality of the measurement properties (based on the Cochrane Back Review Group 200326)

\begin{tabular}{|l|l|l|}
\hline \multicolumn{1}{|c|}{ Level } & \multicolumn{1}{|c|}{ Rating } & \multicolumn{1}{c|}{ Criteria } \\
\hline Strong & +++ or --- & Consistent findings in multiple studies of good methodological quality \\
\hline & & OR in one study of excellent methodological quality \\
\hline Moderate & ++ or -- & Consistent findings in multiple studies of fair methodological quality \\
\hline & & OR in one study of good methodological quality \\
\hline Limited & + or - & One study of fair methodological quality \\
\hline Conflicting & \pm & Conflicting findings \\
\hline Unknown & $?$ & Only studies of poor methodological quality \\
\hline
\end{tabular}

$a+=$ positive results, $?=$ indeterminate results, $-=$ negative results. 


\section{RESULTS}

\section{Description of the included studies}

The systematic search resulted in 2'467 references. After screening of titles and abstracts, 70 potentially relevant full-text articles remained. Finally, 42 studies with 27 different outcome measures (22 capacity and 5 performance measures) met the inclusion criteria (Figure 2.1.).

The characteristics of the studies are summarized in Tables 2.3. and 2.4. All available capacity and performance measurement tools, including their clinical utility, are shown in supplemental Table 2.1. Some studies evaluated measurement properties of different outcome measures and, therefore, are mentioned multiple times in Tables 2.3. and 2.4. ${ }^{27-35}$ Reliability was evaluated in 35 studies, 13 studies dealt with measurement error, and 10 studies examined responsiveness. Children with CP were most prevalent in the assessed studies $(n=26)$, followed by Duchenne muscular dystrophy (DMD) $(n=3)$, Down syndrome (DS) $(n=2)$, and acquired brain injury $(n=2)$. Children with stroke, spina bifida, developmental coordination disorder, and spinal muscular atrophy were each represented in one study, whereas five studies evaluated measures of gait function in mixed patient groups. The Gross Motor Function Measure (GMFM) dimension E $(n=7)$, gait speed $(n=6)$, cadence $(n=5)$, Timed "Up \& Go" Test (TUG) $(n=4)$, and 6MinWT $(n=4)$ were the most frequently evaluated measures of gait function.

\section{Measurement properties}

All results concerning the methodological quality of the studies are based on the modified COSMIN checklist without the sample size requirements. The methodological quality of 16 of the 35 studies evaluating reliability was rated as "good", 16 as "fair," and three as "poor" (Tab. 2.3.). Reasons for a "poor" rating were no or sparse description of the study population and study procedures ${ }^{36}$ and flaws in the statistical analysis, as judged based on criteria of the COSMIN checklist. ${ }^{37,38}$ 


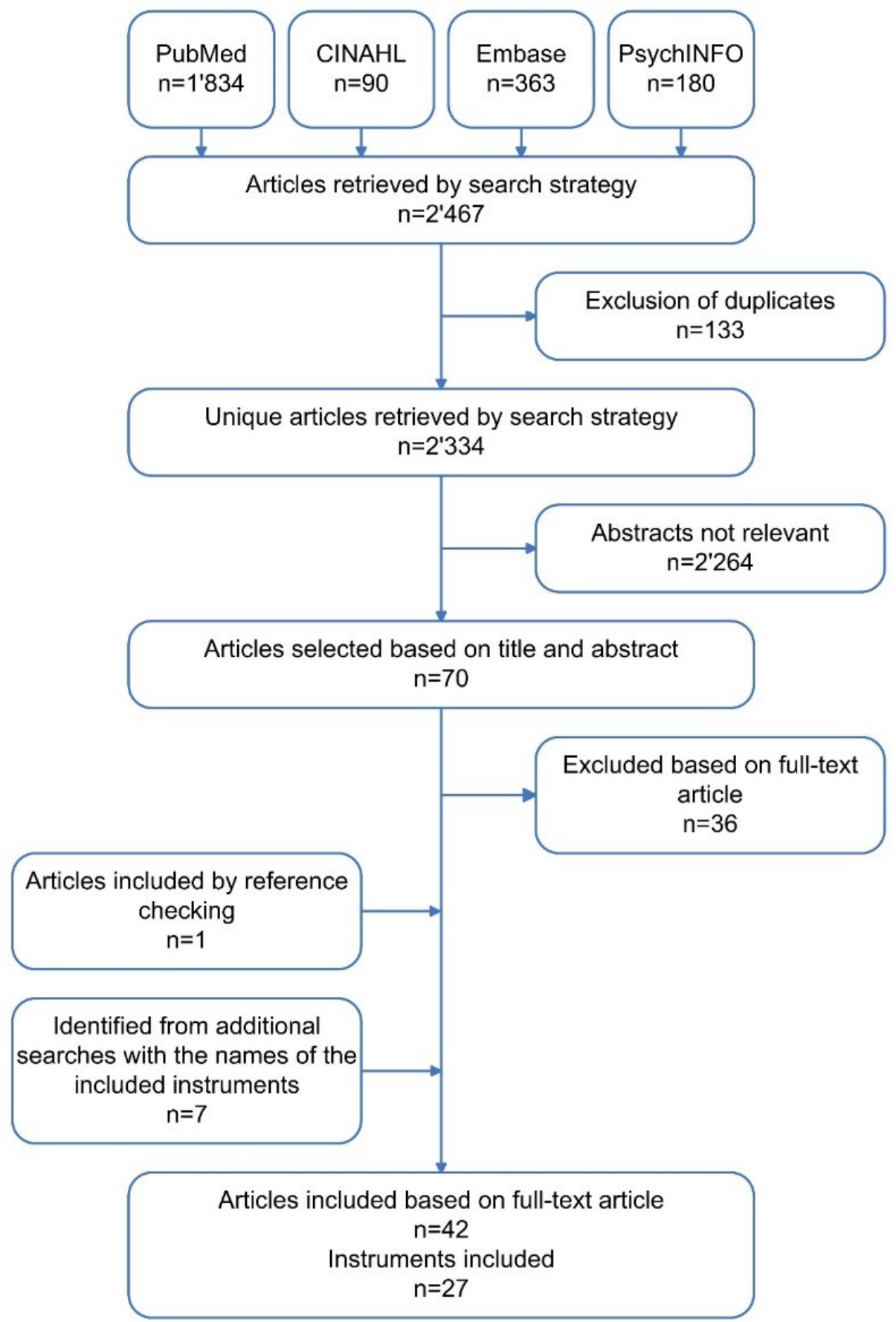

Figure 2.1. Flowchart of the literature search and the selection of the studies. 
Test-retest reliability (the consistency of a measure from one time to another) was evaluated 27 times, interrater reliability (the degree of agreement among different raters) was evaluated 15 times, and intrarater reliability (the degree of agreement among multiple repetitions of a test performed by a single rater) was evaluated eight times. Most studies reported a positive result when the quality criteria concerning the reliability coefficients displayed in Table 2.1. were applied.

Only three studies showed negative reliability results: One study evaluated gait speed of children with developmental coordination disorder on the GAITRite gait analysis system (CIR Systems Inc, Sparta, New Jersey), ${ }^{30}$ one study investigated the gross motor domain of the Paediatric Stroke Activity Limitation Measure in children with stroke, ${ }^{39}$ and one study evaluated the balance subset of the BruininksOseretsky test, the full-turn test, and the TUG in children with DS. ${ }^{29}$ However, this latter study was rated as "poor" and was not taken into account for best evidence synthesis.

\section{Measurement error}

In the 13 studies concerning the measurement error, the quality was rated as "good" in nine studies and as "fair" in four studies (Tab. 2.3.). Information on measurement error was available for the 6MinWT, ${ }^{27,28,41}$ the Shuttle Run Test, ${ }^{42,44}$ the $10 \times 5$-Meter Sprint Test, ${ }^{43}$ the 10 -Meter Fast Walk Test, ${ }^{28}$ the Fast-1-Minute Walk Test, ${ }^{48}$ the GAITRite, ${ }^{31,46}$ the Community Balance and Mobility Scale, ${ }^{47}$ the Functional Walking Test, ${ }^{49}$ gait speed, ${ }^{50}$ the maximal speed during the Treadmill Walking Test, ${ }^{27}$ and the 28-item and 47-item Mobility Questionnaire (MobQues28 and MobQues47). ${ }^{32}$

\section{Responsiveness}

Of the 10 studies on responsiveness, one study was rated as "good," four studies were considered "fair," and five studies were rated as "poor" (Tab. 2.4.). Reasons for a "poor" rating - based on criteria of the COSMIN checklist - were the use of inappropriate statistical methods in all five studies, the lack of a comparator instrument, ${ }^{33,34,65,66}$ and no clear description of the assessment protocol. ${ }^{33,34}$ The studies with a "poor" rating concerned the $6 \mathrm{MinWT},{ }^{65}$ cadence and gait speed measured with the GAITRite ${ }^{34}$ and 3-dimensional gait analysis, ${ }^{33}$ the dimension E of the GMFM, ${ }^{33,67}$ and the Functional Mobility Scale (FMS). ${ }^{66}$ 


\section{Best evidence synthesis}

Results of the best evidence synthesis are summarized in Table 2.5.

Reliability

Given the large diversity of outcome measures and studied patient populations, results could rarely be combined. There was strong positive evidence for interrater reliability of the FMS in children with CP. ${ }^{63,64}$ Moderate positive evidence was shown for test-retest reliability of the $6 \mathrm{MinWT}^{28,41}$ and the TUG57,69 in children with CP. Similarly, moderate positive evidence was found for intrarater reliability of the Functional Walking Test ${ }^{49}$ and the MobQues28 and MobQues4732 as well as for interrater reliability of the MobQues28/47 in children with CP. ${ }^{32}$ Limited positive evidence for reliability in children with $\mathrm{CP}$ was available for the following instruments: the $10 \times 5$-Meter Sprint Test, ${ }^{43}$ the 10 -Meter Fast Walk Test, ${ }^{28}$ the ABILOCO-Kids, ${ }^{45}$ cadence measured with the GAITRite, ${ }^{31,46}$ gait speed measured with a stopwatch, 50 and the Quality Analog Scale. ${ }^{55}$ Also, limited positive evidence for reliability was available for the Community Balance and Mobility Scale in children with acquired brain injury ${ }^{47}$ and the walking scale of the Functional Assessment Questionnaire in children with mixed neuromuscular diagnoses. ${ }^{62}$

\section{Responsiveness}

Moderate negative evidence was available for responsiveness of the dimension $E$ of the GMFM in children with DS. However, when - in addition to the standard scoring procedure - the child's caregiver was asked for information regarding activities of the child demonstrated at home but failed to perform during the assessment (reported score), there was moderate positive evidence for responsiveness instead. ${ }^{51}$ Furthermore, limited positive evidence was available for responsiveness of the $\mathrm{FMS}^{63}$ and the dimension $\mathrm{E}$ of the $\mathrm{GMFM}^{53}$ in children with $\mathrm{CP}$. 
Table 2.3. Characteristics of the included studies (reliability and measurement error)

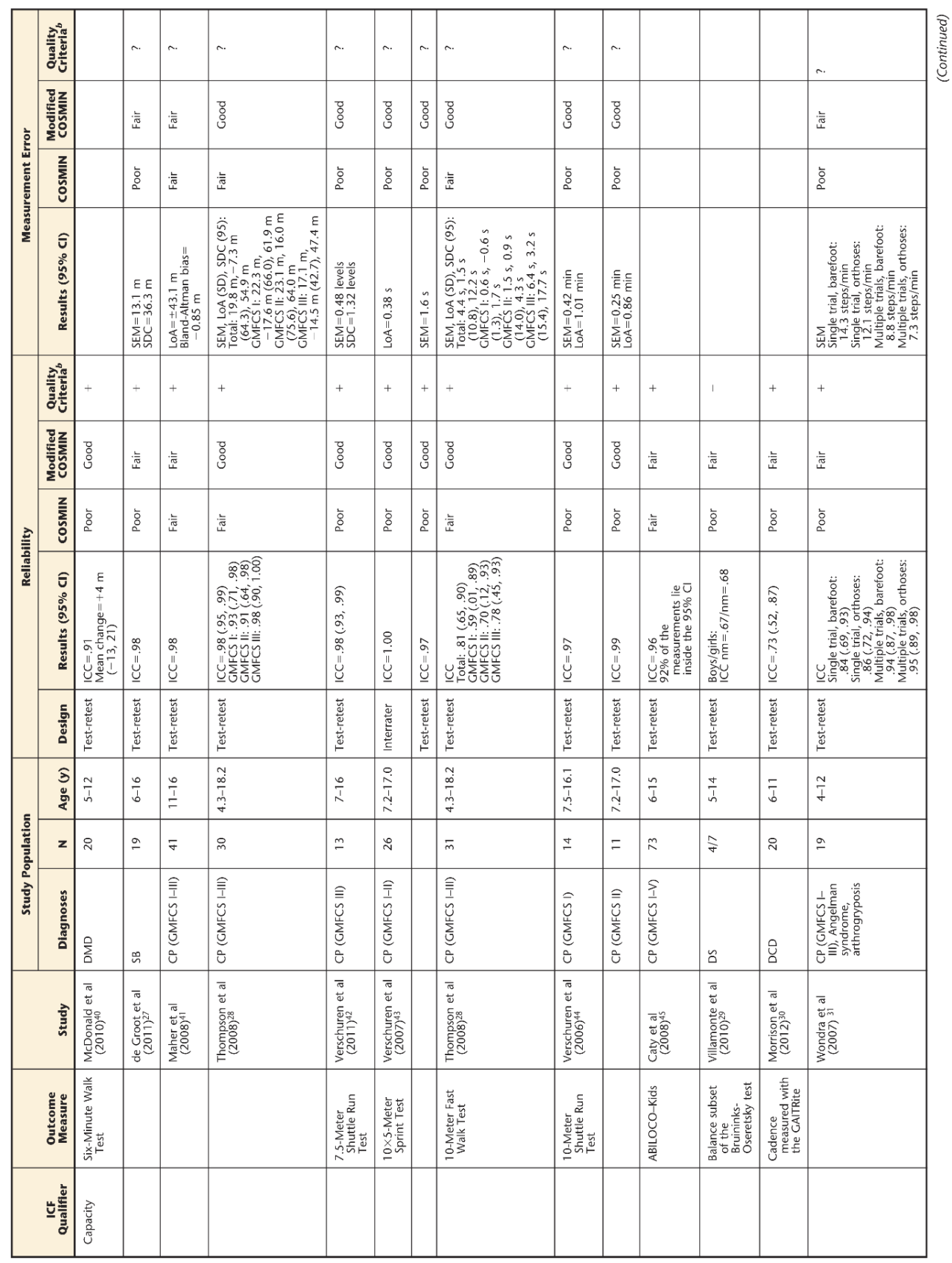


Table 2.3. Continued

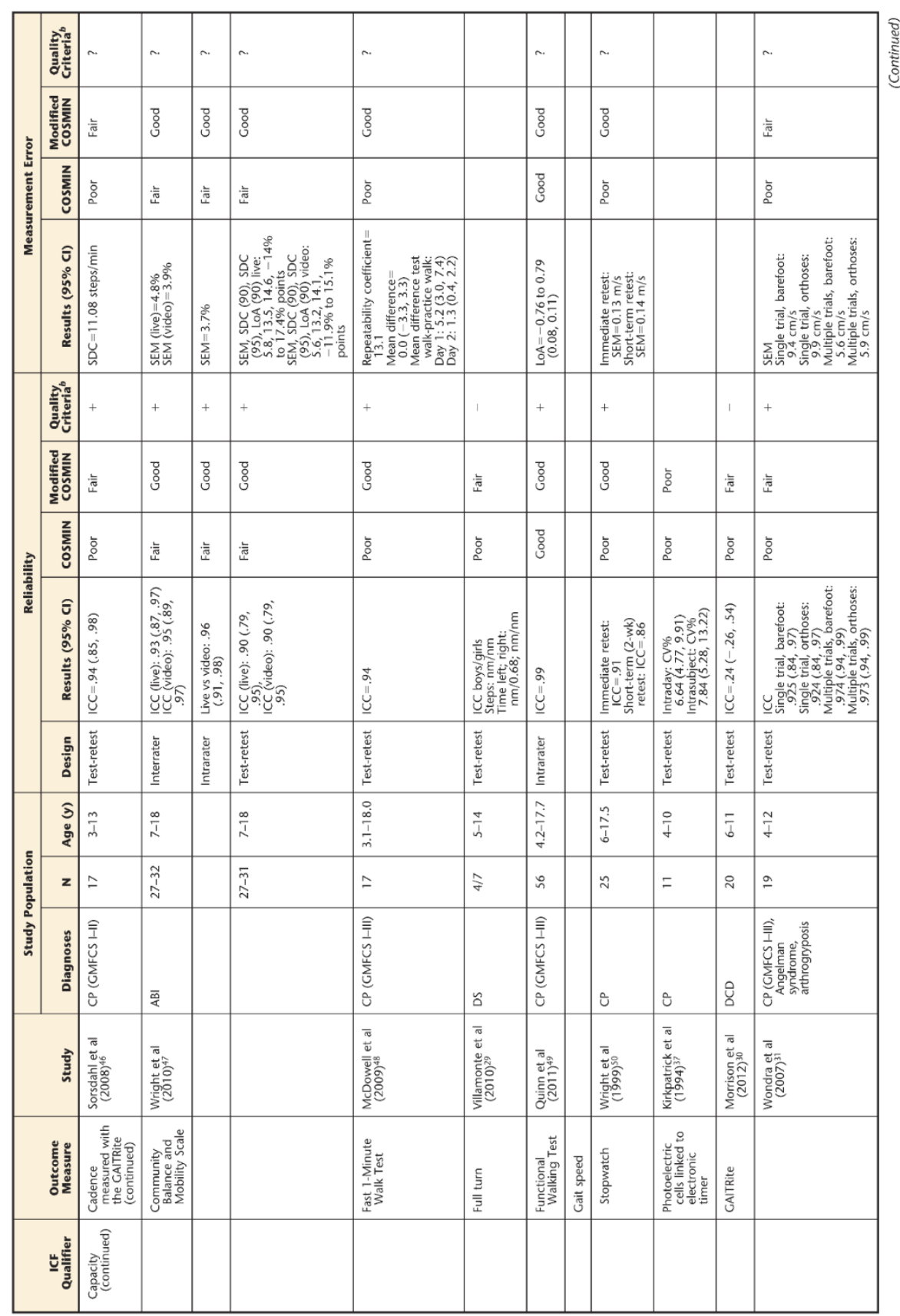


Table 2.3. Continued

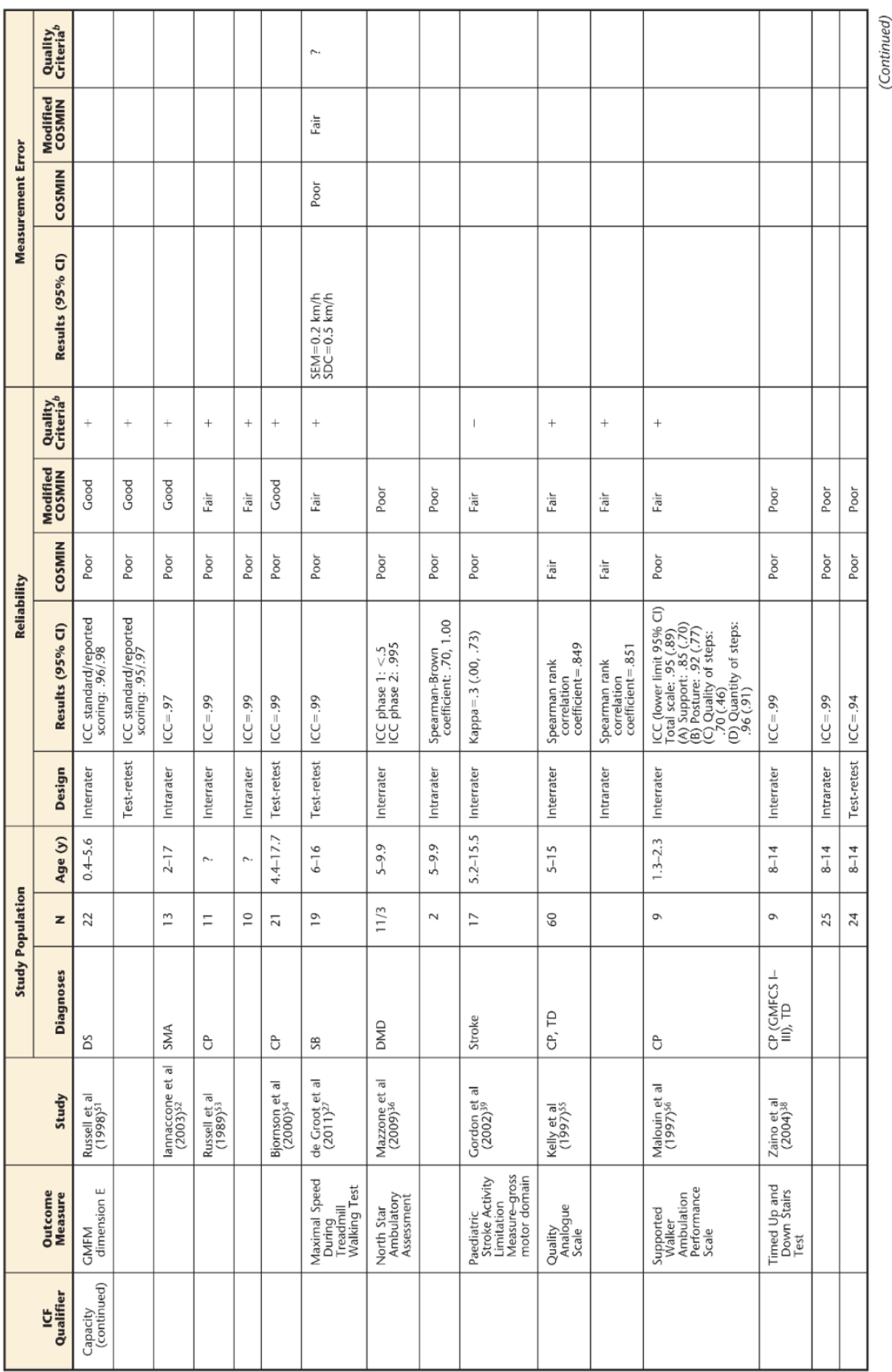


Table 2.3. Continued

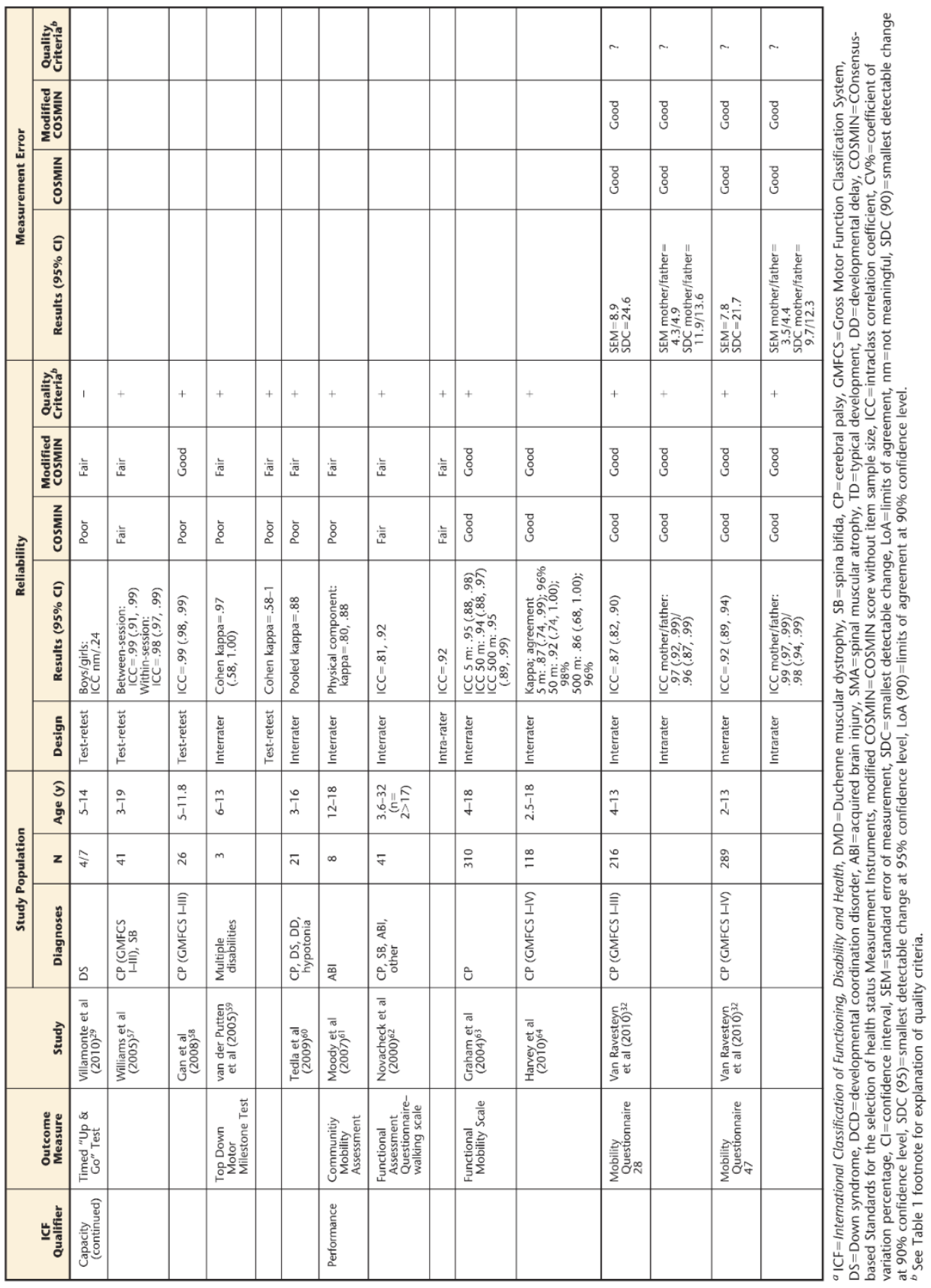


Table 2.4. Characteristics of the included studies (responsiveness)

\begin{tabular}{|c|c|c|c|c|c|c|c|c|c|}
\hline \multirow{2}{*}{$\begin{array}{c}\text { ICF } \\
\text { Qualifier }\end{array}$} & \multirow{2}{*}{$\begin{array}{l}\text { Outcome } \\
\text { Measure }\end{array}$} & \multirow[b]{2}{*}{ Study } & \multicolumn{3}{|c|}{ Study Population } & \multirow{2}{*}{$\begin{array}{c}\text { Responsiveness } \\
\text { Results } \\
\end{array}$} & \multirow[b]{2}{*}{ COSMIN } & \multirow{2}{*}{$\begin{array}{c}\text { Modified } \\
\text { Cosmin }\end{array}$} & \multirow{2}{*}{$\begin{array}{l}\text { Quality } \\
\text { Criteriat }\end{array}$} \\
\hline & & & Diagnoses & N & Age (y) & & & & \\
\hline \multirow[t]{14}{*}{ Capacity } & $\begin{array}{l}\text { Six-Minute Walk } \\
\text { Test }\end{array}$ & $\begin{array}{l}\text { McDonald et } \\
\text { al }(2010)^{66}\end{array}$ & DBMD & 18 & $4-12$ & $\begin{array}{l}\text { Mean 6MWD (range): } 357 \mathrm{~m}(125-481) \\
\text { to } 300 \mathrm{~m}(0-510) \\
6 \mathrm{MWD} \text { decreased in } 12 \text { of } 18 \text { patients } \\
(67 \%) \text {. } \\
\text { Mean change: }-57 \mathrm{~m}(-15.9 \%) \\
\text { Stride length: }-0.13 \mathrm{~m}(-14.6 \%) \\
\text { Cadence: }-11 \text { strides } / \min (-16.9 \%)\end{array}$ & Poor & Poor & \\
\hline & Cadence & & & & & & & & \\
\hline & 3DGA & $\begin{array}{l}\text { Oeffinger et al } \\
(2008)^{33}\end{array}$ & CP (GMFCS I-III) & 381 & $4.1-18.3$ & $\begin{array}{l}\text { SRM GMFCS I, II, III nonsurgical/surgical: } \\
0.03,0.01,0.34 /-0.23,0.07,0.07\end{array}$ & Poor & Poor & \\
\hline & GAITRite & $\begin{array}{l}\text { Kelly et al } \\
(2008)^{34}\end{array}$ & CP (GMFCS I-II) & 9 & $2.2-6.3$ & ES: 0.89 & Poor & Poor & \\
\hline & Gait speed & & & & & & & & \\
\hline & 3DGA & $\begin{array}{l}\text { Oeffinger et al } \\
(2008)^{33}\end{array}$ & CP (GMFCS I-III) & 381 & $4.1-18.3$ & $\begin{array}{l}\text { SRM GMFCS I, II, III nonsurgical surgical: } \\
\quad-0.04,0.04,-0.08 /-0.15,-0.37,0.13\end{array}$ & Poor & Poor & \\
\hline & GAlTRite & $\begin{array}{l}\text { Kelly et al } \\
(2008)^{34}\end{array}$ & CP (GMFCS I-II) & 9 & $2.2-6.3$ & ES: -0.77 & Poor & Poor & \\
\hline & GMFM dimension $\mathrm{E}$ & $\begin{array}{l}\text { Russell et al } \\
\quad(1998)^{51}\end{array}$ & DS & $29-117$ & $0.1-6$ & $\begin{array}{l}\text { Correlations of GMFM dimension E } \\
\text { change score with change as judged by } \\
\text { parent/intervenor/video rating: } \\
\text { Standard scoring: } 44 / .48 / .46 \\
\text { Reported scoring: } 0.56 / .59 / .40\end{array}$ & Good & Good & $-1++^{c}$ \\
\hline & & $\begin{array}{l}\text { Russell et al } \\
(1989)^{53}\end{array}$ & $C P, A B I, T D$ & 170 & $<3-20$ & $\begin{array}{l}\text { Correlations between change on GMFM } \\
\text { and change judged by physical } \\
\text { therapistssparents/blind video analysis: } \\
.74 / .68 / .52\end{array}$ & Fair & Fair & + \\
\hline & & $\begin{array}{l}\text { Nordmark et } \\
\text { al }(2000)^{68}\end{array}$ & CP (GMFCS I-V) & 18 & $2.5-6.0$ & $\begin{array}{l}P \text { value at } 0-6 \mathrm{mo} / 6-12 \mathrm{mo} / 0-12 \mathrm{mo}: \\
\text { Total: }-/-/- \text { - } \\
\text { Moderate/middle group: }-1-1.05 \\
\text { Severe group: }-/-/-\end{array}$ & Poor & Poor & \\
\hline & & $\begin{array}{l}\text { Vos-Vromans } \\
\text { et al } \\
(2005)^{69}\end{array}$ & CP (GMFCS I-II) & 55 & $2-7.2$ & $\begin{array}{l}\text { Correlations between GMFM dimension E } \\
\text { change score and change in global } \\
\text { rating scale score for parents total } \\
\text { group/younger group/older group: } \\
.46(P<01) / .41 \text { (ns) } / .43 \quad P<05) \\
\text { ES: } 0.71 / 1.16 / 0.67 \\
\text { SRM: } 1.42 / 2.38 / 1.56\end{array}$ & Fair & Fair & $?$ \\
\hline & & $\begin{array}{l}\text { Oeffinger et al } \\
(2008)^{33}\end{array}$ & CP (GMFCS I-III) & 381 & $4.1-18.3$ & $\begin{array}{l}\text { SRM GMFCS } 1, \| 1, \text { III nonsurgical/surgical: } \\
\quad-0.03,0.18,0.69 / 0.04,-0.36,-0.04\end{array}$ & Poor & Poor & \\
\hline & $\begin{array}{l}\text { Timed Up and } \\
\text { Down Stairs Test }\end{array}$ & $\begin{array}{l}\text { de Campos et } \\
\text { al }(2011)^{35}\end{array}$ & CP (GMFCS I-II) & 6 & $5-12$ & $\begin{array}{l}\text { Spearman correlations: } \\
\text { TUDS-GMFM dimension D/GMFM } \\
\text { dimension E: } r=-.58(P=.05) / \\
r=-.66(P=.02)\end{array}$ & Poor & Fair & + \\
\hline & 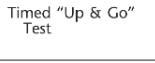 & $\begin{array}{l}\text { de Campos et } \\
\text { al }(2011)^{35}\end{array}$ & CP (GMFCS I-II) & 6 & $5-12$ & $\begin{array}{l}\text { Spearman correlations: } \\
\text { TUG-GMFMD/GMFME: } r=-.47(P=.12) / \\
r=-.71(P=.009)\end{array}$ & Poor & Fair & + \\
\hline \multirow[t]{2}{*}{ Performance } & $\begin{array}{l}\text { Functional Mobility } \\
\text { Scale }\end{array}$ & $\begin{array}{l}\text { Graham et al } \\
(2004)^{63}\end{array}$ & $\mathrm{CP}$ & 35 & $4-18$ & $\begin{array}{l}6 \mathrm{mo} / 12 \mathrm{mo:} \\
\text { PODCl } 5 \mathrm{~m}, 50 \mathrm{~m}, 500 \mathrm{m:} \\
0.78,0.82,0.81 / 0.79,0.81,0.80 \\
\text { CHO } 5 \mathrm{~m}, 50 \mathrm{~m}, 500 \mathrm{m:}: \\
0.77,0.80,0.80 / 0.75,0.82,0.83 \\
\text { EE } 5 \mathrm{~m}, 50 \mathrm{~m}, 500 \mathrm{m:} 0.53,0.59,0.52 / \\
0.54,0.55,0.53 \mathrm{~m}, 500 \mathrm{~m}: 0.86,0.84 \\
\text { Up time } 5 \mathrm{~m}, 50 \mathrm{~m}, \\
0.85 / 0.78,0.85,0.89\end{array}$ & Fair & Fair & + \\
\hline & & $\begin{array}{l}\text { Harvey et al } \\
(2007)^{67}\end{array}$ & CP (GMFCS I-III) & 66 & $6-16$ & $\begin{array}{l}\text { OR (Cl) } 3 \mathrm{mo}^{6} 6 \mathrm{mo}, 9 \mathrm{mo}, 12 \mathrm{mo}, 24 \mathrm{mo}: \\
5 \mathrm{m:} 0.13(0.07,0.24), 0.36(0.23 \mathrm{c} 0.58) \\
0.69(0.45,1.04), 1.12(0.77,1.64) \\
2.08(1.33,3.24) \\
50 \mathrm{~m}: 0.09(0.04,0.17) \\
0.32(0.19,0.55), 0.77(0.49,1.19) \\
1.22(0.82,1.81), 2.16(1.37,3.41) \\
500 \mathrm{~m}: 0.24(0.14,0.43), \\
0.50(0.32,0.80), 0.90(0.57,1.41) \\
1.47(0.97,2.22), 2.23(1.44,3.45)\end{array}$ & Poor & Poor & \\
\hline
\end{tabular}

${ }^{a} \mathrm{ICF}=$ International Classification of Functioning, Disability and Health, DBMD=Duchenne/Becker muscular dystrophy, CP=cerebral palsy, GMFCS $=\mathrm{Gross}$ Motor Function Classification System, DS=Down syndrome, ABI=acquired brain injury, TD=typical development, COSMIN=COnsensus-based Standards for the selection of health status Measurement Instruments, modified COSMIN=COSMIN score without item sample size, ES=effect size, SRM=standardized response mean, $\mathrm{OR}=$ odds ratio, $\mathrm{Cl}=$ confidence interval, $6 \mathrm{MWD}=$ distance walked during the Six-Minute Walk Test, GMFM=Gross Motion Function Measure, TUDS=Timed Up and Down Stairs Test, TUG=Timed "Up \& Go" Test, FMS=Functional Mobility Scale, PODCI=Pediatric Outcomes Data Collection Instrument (physical function and sports domains), $\mathrm{CHQ}=$ Child Health Questionnaire-Australian version (physical functioning domain), $\mathrm{EE}=$ energy expenditure, $\mathrm{ns}=$ not significant, $3 \mathrm{DCA}=3$-dimensional gait analysis.

${ }^{b}$ See Table 1 footnote for explanation of quality criteria.

c Standard/reporting scoring. 
Table 2.5. Best evidence synthesis

\begin{tabular}{|c|c|c|c|c|c|c|}
\hline \multirow[b]{2}{*}{$\begin{array}{c}\text { ICF } \\
\text { Qualifier }\end{array}$} & \multirow[b]{2}{*}{ Outcome Measure } & \multicolumn{5}{|c|}{ Reliability } \\
\hline & & Test-Retest & Interrater & Intrarater & $\begin{array}{c}\text { Measurement } \\
\text { Error }\end{array}$ & Responsiveness \\
\hline \multirow[t]{23}{*}{ Capacity } & Six-Minute Walk Test ${ }^{27,28,40,41}$ & $\begin{array}{l}++\mathrm{CP} \\
? \mathrm{DMD}, \mathrm{SB}\end{array}$ & & & $\begin{array}{l}\text { ? CP } \\
\text { ? SB }\end{array}$ & \\
\hline & 7.5-Meter Shuttle Run Test ${ }^{42}$ & ? CP & & & ? CP & \\
\hline & $10 \times 5$-Meter Sprint Test ${ }^{43}$ & $+\mathrm{CP}$ & $+\mathrm{CP}$ & & $? \mathrm{CP}$ & \\
\hline & 10-Meter Fast Walk Test ${ }^{28}$ & $+\mathrm{CP}$ & & & $? \mathrm{CP}$ & \\
\hline & 10-Meter Shuttle Run Test ${ }^{44}$ & ? CP & & & ? CP & \\
\hline & ABILOCO-Kids ${ }^{45}$ & $? \mathrm{CP}$ & & & & \\
\hline & $\begin{array}{l}\text { Balance subset of the Bruininks- } \\
\text { Oseretsky test }{ }^{29}\end{array}$ & ?DS & & & & \\
\hline & $\begin{array}{l}\text { Cadence measured with } \\
\text { GAITRite }{ }^{30,31,46}\end{array}$ & $\begin{array}{l}+\mathrm{CP} \\
? \mathrm{DCD}\end{array}$ & & & $? \mathrm{CP}$ & \\
\hline & $\begin{array}{l}\text { Community Balance and } \\
\text { Mobility Scale }{ }^{47}\end{array}$ & $+\mathrm{ABI}$ & $+\mathrm{ABI}$ & $+A B I$ & ? ABI & \\
\hline & Fast 1-Minute Walk Test ${ }^{48}$ & $? \mathrm{CP}$ & & & $? \mathrm{CP}$ & \\
\hline & Full turn ${ }^{29}$ & ?DS & & & & \\
\hline & Functional Walking Test ${ }^{49}$ & & & $++\mathrm{CP}$ & $? \mathrm{CP}$ & \\
\hline & Gait speed & & & & & \\
\hline & Stopwatch ${ }^{50}$ & $+\mathrm{CP}$ & & & $? \mathrm{CP}$ & \\
\hline & GAITRite 30,31 & ? CP, DCD & & & $? \mathrm{CP}$ & \\
\hline & GMFM dimension $\mathrm{E}^{51-54,69}$ & ?CP, DS & ?CP, DS & ? CP, SMA & & $\begin{array}{l}+\mathrm{CP} \\
--1++\mathrm{DS}^{b}\end{array}$ \\
\hline & $\begin{array}{l}\text { Maximal Speed During } \\
\text { Treadmill Walking Test }{ }^{27}\end{array}$ & $? S B$ & & & ? SB & \\
\hline & $\begin{array}{l}\text { Paediatric Stroke Activity } \\
\text { Limitation Measure-gross } \\
\text { motor domain } 39\end{array}$ & & ? Stroke & & & \\
\hline & Quality Analogue Scale ${ }^{5 S}$ & & $+\mathrm{CP}$ & $+\mathrm{CP}$ & & \\
\hline & $\begin{array}{l}\text { Supported Walker Ambulation } \\
\text { Performance Scale }\end{array}$ & & ? CP & & & \\
\hline & $\begin{array}{l}\text { Timed Up and Down Stairs } \\
\text { Test }{ }^{35}\end{array}$ & & & & & $? \mathrm{CP}$ \\
\hline & Timed "Up \& Go" Test29,35,57,58 & $\begin{array}{l}++\mathrm{CP} \\
? \mathrm{DS}\end{array}$ & & & & $? \mathrm{CP}$ \\
\hline & $\begin{array}{l}\text { Top Down Motor Milestone } \\
\text { Test } 59,60\end{array}$ & ? Mixed & ? Mixed & & & \\
\hline \multirow[t]{4}{*}{ Performance } & $\begin{array}{l}\text { Community Mobility } \\
\text { Assessment }{ }^{61}\end{array}$ & & $? A B I$ & & & \\
\hline & $\begin{array}{l}\text { Functional Assessment } \\
\text { Questionnaire-walking scale }{ }^{62}\end{array}$ & & + Mixed & + Mixed & & \\
\hline & Functional Mobility Scale ${ }^{63,64}$ & & $+++\mathrm{CP}$ & & & $+\mathrm{CP}$ \\
\hline & Mobility Questionnaire $28 / 47^{32}$ & & $++\mathrm{CP}$ & $++\mathrm{CP}$ & ? CP & \\
\hline
\end{tabular}

${ }^{a} \mathrm{ICF}=$ International Classification of Functioning, Disability and Health, DMD=Duchenne muscular dystrophy, $\mathrm{SB}=$ spina bifida, $\mathrm{CP}=\mathrm{cerebral}$ palsy, $\mathrm{DS}=\mathrm{Down}$ syndrome, $\mathrm{DCD}=$ developmental coordination disorder, $\mathrm{ABI}=$ acquired brain injury, $\mathrm{SMA}=$ spinal muscular atrophy, mixed=multiple disabilities,

$+++/---=$ strong positive/negative evidence, $++/--=$ moderate positive/negative evidence, $+/-$ limited positive/negative evidence, ?=unknown.

${ }^{b}$ Standard/reported scoring.

\section{DISCUSSION}

The purpose of this systematic literature review was to evaluate the evidence on reliability, measurement error, and responsiveness of functional measures of gait in children and youth with neuromuscular diagnoses. We identified 42 eligible papers 
evaluating the measurement properties of 27 different measures of gait function. These measures covered variable constructs, such as walking ability, functional mobility, gait speed, dynamic balance, and activity limitations - all being an integral part of gait function. Some authors also mentioned constructs such as aerobic fitness, power, and agility (supplemental Table 2.1.). However, although the authors interpreted their results reflecting these constructs, all the tests in question are just measures of mobility combining speed and endurance, as patients have to walk as fast or as long, or both, as possible.

Most of the studies were rated as "good" or "fair" on the 4-point rating scale of the modified COSMIN checklist without sample size, whereas eight studies were judged as being of poor quality and had to be excluded from the best evidence synthesis process. Responsiveness studies mostly suffered from a lack of quality, with five out of 10 studies being classified as "poor". According to the COSMIN manual, "the responsiveness issue is about whether the direction and magnitude of a correlation is similar to what could be expected based on the construct(s) that are being measured."70 This statement implies that hypotheses on the relationship of different measurement instruments have to be stated and tested. This requirement was not fulfilled in any of the five studies with a "poor" rating. By excluding studies with poor quality from the best evidence synthesis, the North Star Ambulatory Assessment, an outcome measure specifically developed for children with DMD, was not represented in the best evidence synthesis anymore. ${ }^{36}$

Measurement properties still need to be determined for most instruments and most diagnoses. Not surprisingly, most evidence was available for children with $\mathrm{CP}$, as these children also represent the largest fraction in the neuropediatric field. Nevertheless, even for these children, there was no measurement tool with evidence reported for all three measurement properties that were evaluated in this review. The instrument showing most information on measurement properties was the FMS, with strong positive evidence for reliability and limited positive evidence for responsiveness in children with CP. Although capacity measures strongly dominated, the FMS represents one of the few performance measures. Interestingly, far more studies evaluated the reliability than the measurement error. For evaluative 
measures, information on the agreement, which is quantified with the measurement error, is much more valuable than reliability. Whereas reliability parameters highly depend on the variation in the population sample, agreement is more a characteristic of the measurement instrument itself. As such, it allows the conclusion whether a change in score represents a real change. Because the measurement error is expressed in the unit of the measurement, it also facilitates the clinical interpretation of a change score. ${ }^{71}$

In general, results from different studies could rarely be combined. The GMFM dimension $\mathrm{E}$, for example, was the instrument being most frequently represented in this review, with seven different publications. Nevertheless, its reliability remains undetermined because the patient samples were too small and heterogeneous; therefore, a synthesis of different studies was not feasible. The poor possibilities of combining different study results are a problem also faced by other authors of systematic reviews on measurement properties. ${ }^{17,23}$ Main reasons are inhomogeneous patient populations, too large variation in test procedures, or just singular publications per outcome measure. In this review, some studies with mixed patient groups were included. In cases where the predominant diagnosis was CP and other diagnoses accounted for only a small portion of the study population, we combined the results for the best evidence synthesis. This procedure concerned studies on reliability of the TUG 57,69 and of the cadence measured with the GAITRite. ${ }^{31,46}$

Although different approaches exist to assess the quality of studies, the COSMIN checklist has increasingly been used in systematic reviews on measurement properties of health measurement instruments in the last few years. Also, some of the systematic reviews on measurement properties in the pediatric field used the COSMIN checklist. ${ }^{12,14,17}$ This allows for a comparison of the performed quality ratings concerning the congruent studies and measurement properties across the different reviews. Eight studies included in our review were also rated in the systematic review of Adair et al. ${ }^{14}$ When the scoring (with the inclusion of the sample size item) was compared, four studies ${ }^{33,53,54,64}$ achieved an identical score, three studies $^{52,63,66}$ differed by one level, and the quality rating of one study remained 
unclear $^{62}$ in the review of Adair et al. Reasons for the scoring differences are hard to find, as Adair et al. provided an overall score rather than a score per item. The ratings of Balemans et al. ${ }^{12}$ are difficult to compare because they used an older version of the COSMIN checklist, which requires only a "yes" (adequate) or "no" (not adequate) rating in comparison with the 4-point rating scale used in this review. Finally, the review of Bartels et al. on measurement properties of the $6 \mathrm{MinWT}^{17}$ yielded five studies for a possible comparison, two of them being identically scored in the two reviews, ${ }^{41,65}$ whereas three differed by one level. ${ }^{27,28,40}$ Differences were related to at least two items per study as well as to the overall rating of each study. In all of these studies, a systematic discrepancy could not be detected between the ratings. The interrater agreement and reliability of the COSMIN checklist have already been investigated. ${ }^{72}$ Nevertheless, conclusions have to be drawn with precaution, as reliability has only been determined on item level and without the 4-point rating option, which has just recently been developed ${ }^{21}$ and was used in this review. To increase the interrater agreement, the developers of the checklist recommend gaining some experience with the checklist before conducting a systematic review and finding consensus on how to score items that require some subjective judgement in advance. To follow these recommendations, we pretested three articles, discussed problems that occurred, and defined a joint procedure in items containing subjective issues. Furthermore, we strictly adhered to the taxonomy and terminology of the COSMIN checklist. Comparing the evidence for measurement tools that were also evaluated in other reviews, there is consensus that positive evidence exists for reliability ${ }^{10,14}$ and responsiveness ${ }^{14}$ of the FMS in children with CP as well as for reliability of the Functional Assessment Questionnaire in children with mixed neuromuscular diagnoses. ${ }^{10,14}$ Concerning the 6MinWT, we found only positive evidence for reliability in children with CP. Bartels et al. ${ }^{17}$ also reported a positive quality rating for reliability in children with DMD and spina bifida. There were also discrepancies regarding the various shuttle run tests. We concluded that evidence for all evaluated psychometric properties is unknown; in contrast to this conclusion, Balemans et al. ${ }^{12}$ found excellent positive evidence for reliability and measurement error in those tests. 
Several reasons exist for the differences in the reported evidence levels. Not all authors accounted for the methodological quality of the included studies when summarizing the evicence, ${ }^{10,14}$ or requirements regarding the quality criteria for the measurement properties were not stated. ${ }^{14}$ Balemans et al. rated the minimal important change of a measure based on their clinical experience when it was not stated in a study, ${ }^{12}$ whereas we rated the evidence as unknown in those cases. Furthermore, requirements concerning the sample size had not been established ${ }^{10,12,14}$ or were different than in our review. ${ }^{17}$

\section{Study limitations and methodological considerations}

The COSMIN checklist was originally developed to evaluate the methodological quality of studies on measurement properties of patient-reported questionnaires. Nevertheless, these standards can also be applied to other outcomes, such as performance-based tests or rating scales. By excluding the sample size item from the quality assessment and accounting for it in the best evidence synthesis stage, we deviated from the standard COSMIN procedure. Given that sample size is usually rather small in neuropediatric studies, the available evidence would have been drastically reduced by more than $50 \%$ without this approach. Two studies included in the best evidence synthesis were performed with mixed patient groups ${ }^{31,57}$ and were combined with studies specifically investigating children with CP. As the number of patients with a diagnosis other than CP was rather small and all children had a chronic, congenital condition, we assume that the results found for the mixed patient group well reflected the results of the children diagnosed with $\mathrm{CP}$. Nonetheless, if this assumption was not true, the level of evidence for the concerning outcome tools (TUG and cadence measured with the GAITRite) could be biased. We decided to concentrate on reliability, measurement error, and responsiveness, as these are the most critical measurement properties for an evaluative measurement tool. As we did not include articles evaluating the validity of functional measures of gait, a comprehensive appraisal of the best available measure of gait function per diagnosis was not possible. By excluding papers that were not published in the English or German language, stronger evidence on the measurement properties or information on further measurement tools may have been missed. 
Although we stringently followed the COSMIN guidelines, we did not always agree with the defined standards. Concerning responsiveness, for example, the COSMIN criteria recommend formulating and testing hypotheses on the direction and magnitude of change score correlations between two different measures. Hence, the use of effect sizes or a standardized response mean is considered inappropriate. Although this approach may be reasonable in health-related patient-reported outcomes, we question the clinical sense of this criterion in performance-based measures, as a gold standard hardly exists in these measures.

\section{Implications for clinical practice and research}

The lack of evidence on the measurement properties of many measures assessed in this systematic review does not imply that these tools are not appropriate to assess gait function in children with neuromuscular diagnoses. Rather, it demonstrates the shortage of high-quality studies evaluating the measurement properties of many outcome tools that are already widely used in pediatric neuro-rehabilitation. In a first step, we recommend that rehabilitation specialists and researchers seek dialogue and find consensus on the most relevant measures of gait function and focus the testing of the measurement properties on these outcome tools. Until then, clinicians have to decide on an individual basis which tools are most relevant and feasible for their specific situation. For an integral picture of the child's walking abilities, capacity measures as well as performance measures should be represented. We believe that this review and the description of the tools' characteristics in supplemental Table 2.1. are of help in this selection process.

\section{CONCLUSION}

Many different measures are used to assess gait function in children with neuromuscular conditions. Most of the tools give an indication of the child's capacity level, with only a few considering the performance level. There is moderate to strong evidence on reliability for several measures in children with CP (6MinWT, TUG, FMS, MobQues28 and MobQues47, and Functional Walking Test). Positive evidence for responsiveness exists for the FMS as well as the GMFM dimension E in children with $\mathrm{CP}$. Evidence on measurement error is completely lacking. 
This systematic review highlights the urgent need for high-quality studies evaluating the measurement properties of evaluative outcome tools assessing gait function in children with neuromuscular diagnoses. To facilitate the combination of different study results for best evidence synthesis, specialists from the clinic and research should find consensus on the most appropriate and relevant measures of gait function. Studies on responsiveness and measurement error, including information on the minimal import change, are especially desirable, as they allow a clinically relevant interpretation of a patient's change score. This latter issue is essential, as the success of future trials investigating the effectiveness of interventions aiming at improving gait will strongly depend on the use of appropriate outcome measures.

\section{ACKNOWLEDGMENTS}

The authors thank Dr Martina Gosteli from the main library of the University of Zurich for her comments on the literature search strategy and $\mathrm{Dr}$ Caroline B. Terwee for her valuable help and comments on the appropriate use of the COSMIN guidelines.

\section{FUNDING}

The authors gratefully acknowledge funding by the Mäxi Foundation, Zurich, Switzerland.

\section{REFERENCES}

1. Novak I, Hines M, Goldsmith S, Barclay R. Clinical prognostic messages from a systematic review on cerebral palsy. Pediatrics. 2012;130(5):e1285-312.

2. Lollar DJ, Simeonsson RJ, Nanda U. Measures of outcomes for children and youth. Arch Phys Med Rehabil. 2000;81(12 Suppl 2):S46-52.

3. World Health Organisation. International Classification of Functioning, Disability and Health (ICF). 2001. Available at: http://www.who.int/classifications/icf/en/. Accessed May 30, 2013.

4. World Health Organisation. The International Classification of Functioning, Disability and Health for Children and Youth (ICF-CY) Conference. 2007. Available at: http://www.euro.who.int/en/who-we-are/networks/regions-for-health-network$\mathrm{rhn} /$ regional-health-policy/veneto-italy/the-international-classification-offunctioning,-disability-and-health-for-children-and-youth-icf-cy-conference.

Accessed May 30, 2013. 
5. Holsbeeke L, Ketelaar M, Schoemaker MM, Gorter JW. Capacity, capability, and performance: different constructs or three of a kind? Arch. Phys. Med. Rehabil. 2009;90(5):849-55.

6. Bjornson K, Hays R, Graubert C, Price R. Botulinum toxin for spasticity in children with cerebral palsy: a comprehensive evaluation. Pediatrics. 2007;120(1):49-58.

7. Mokkink LB, Terwee CB, Stratford PW, et al. Evaluation of the methodological quality of systematic reviews of health status measurement instruments. Qual. Life Res. 2009;18(3):313-33.

8. Morris C, Kurinczuk JJ, Fitzpatrick R. Child or family assessed measures of activity performance and participation for children with cerebral palsy: a structured review. Child Care Heal. Dev. 2005;31(4):397-407.

9. Sakzewski L, Boyd R, Ziviani J. Clinimetric properties of participation measures for 5- to 13-year-old children with cerebral palsy: a systematic review. Dev Med Child Neurol. 2007;49(3):232-240.

10. Harvey A, Robin J, Morris ME, Graham HK, Baker R. A systematic review of measures of activity limitation for children with cerebral palsy. Dev Med Child Neurol. 2008;50(3):190-198.

11. Debuse D, Brace H. Outcome measures of activity for children with cerebral palsy: a systematic review. Pediatr Phys Ther. 2011;23(3):221-231.

12. Balemans AC, Fragala-Pinkham $\mathrm{M}$ a, Lennon $\mathrm{N}$, et al. Systematic review of the clinimetric properties of laboratory- and field-based aerobic and anaerobic fitness measures in children with cerebral palsy. Arch. Phys. Med. Rehabil. 2013;94(2):287301.

13. Alderson L, Watson T, Main E. Assessment of dynamic walking balance in children with acquired brain injury. Phys. Ther. Rev. 2007;12(1):3-20.

14. Adair B, Said CM, Rodda J, Morris ME. Psychometric properties of functional mobility tools in hereditary spastic paraplegia and other childhood neurological conditions. Dev Med Child Neurol. 2012;54(7):596-605.

15. Hailey D, Tomie J. An assessment of gait analysis in the rehabilitation of children with walking difficulties. Disabil Rehabil. 2000;22(6):275-280.

16. Young NL, Wright JG. Measuring pediatric physical function. J. Pediatr. Orthop. 1995;15(2):244-53.

17. Bartels B, de Groot JF, Terwee CB. The Six-Minute Walk Test in Chronic Pediatric Conditions: A Systematic Review of Measurement Properties. Phys. Ther. 2013;93(4):529-41.

18. Terwee CB, Jansma EP, Riphagen II, de Vet HCW. Development of a methodological PubMed search filter for finding studies on measurement properties of measurement instruments. Qual. Life Res. 2009;18(8):1115-23. 
19. Mokkink LB, Terwee CB, Patrick DL, et al. The COSMIN checklist for assessing the methodological quality of studies on measurement properties of health status measurement instruments: an international Delphi study. Qual. life Res. 2010;19(4):539-49.

20. Mokkink LB, Terwee CB, Knol DL, et al. The COSMIN checklist for evaluating the methodological quality of studies on measurement properties: A clarification of its content. BMC Med. Res. Methodol. 2010;10(22).

21. Terwee CB, Mokkink LB, Knol DL, Ostelo RWJG, Bouter LM, de Vet HCW. Rating the methodological quality in systematic reviews of studies on measurement properties: a scoring system for the COSMIN checklist. Qual. Life Res. 2012;21(4):651-7.

22. Mokkink LB, Terwee CB, Patrick DL, et al. The COSMIN study reached international consensus on taxonomy, terminology, and definitions of measurement properties for health-related patient-reported outcomes. J. Clin. Epidemiol. 2010;63(7):737-45.

23. Dobson F, Hinman RS, Hall M, Terwee CB, Roos EM, Bennell KL. Measurement properties of performance-based measures to assess physical function in hip and knee osteoarthritis: a systematic review. Osteoarthr. Cartil. 2012;20(12):1548-62.

24. Law M. Measurement in occupational therapy: Scientific criteria for evaluation. Can. J. Occup. Ther. 1987;54(3):133-138.

25. Terwee CB, Bot SDM, de Boer MR, et al. Quality criteria were proposed for measurement properties of health status questionnaires. J. Clin. Epidemiol. 2007;60(1):34-42.

26. Van Tulder M, Furlan A, Bombardier C. Updated Method Guidelines for Systematic Reviews in the Cochrane Collaboration Back Review Group. 2003;28(12):1290-1299.

27. De Groot JF, Takken T, Gooskens RH, et al. Reproducibility of maximal and submaximal exercise testing in "normal ambulatory" and "community ambulatory" children and adolescents with spina bifida: which is best for the evaluation and application of exercise training? Phys Ther. 2011;91(2):267-276.

28. Thompson P, Beath T, Bell J, et al. Test-retest reliability of the 10-metre fast walk test and 6-minute walk test in ambulatory school-aged children with cerebral palsy. Dev Med Child Neurol. 2008;50(5):370-376.

29. Villamonte R, Vehrs PR, Feland JB, Johnson a. W, Seeley MK, Eggett D. Reliability of 16 Balance Tests in Individuals With Down Syndrome 1. Percept. Mot. Skills. 2010;111(2):530-542.

30. Morrison SC, Ferrari J, Smillie S. Are spatiotemporal gait characteristics reliable outcome measures in children with developmental coordination disorder? Pediatr Phys Ther. 2012;24(1):46-50. 
31. Wondra V, Pitetti K, Beets M. Gait parameters in children with motor disabilities using an electronic walkway system: assessment of reliability. Pediatr. Phys. Ther. 2007.

32. Van Ravesteyn NT, Dallmeijer AJ, Scholtes VA, Roorda LD, Becher JG. Measuring mobility limitations in children with cerebral palsy: interrater and intrarater reliability of a mobility questionnaire (MobQues). Dev Med Child Neurol. 2010;52(2):194-199.

33. Oeffinger D, Bagley a, Rogers $\mathrm{S}$, et al. Outcome tools used for ambulatory children with cerebral palsy: responsiveness and minimum clinically important differences. Dev. Med. Child Neurol. 2008;50(12):918-25.

34. Kelly B, MacKay-Lyons MJ, Berryman S, Hyndman J, Wood E. Assessment protocol for serial casting after botulinum toxin a injections to treat equinus gait. Pediatr Phys Ther. 2008;20(3):233-241.

35. De Campos AC, da Costa CS, Rocha NA. Measuring changes in functional mobility in children with mild cerebral palsy. Dev Neurorehabil. 2011;14(3):140-144.

36. Mazzone ES, Messina S, Vasco G, et al. Reliability of the North Star Ambulatory Assessment in a multicentric setting. Neuromuscul Disord. 2009;19(7):458-461.

37. Kirkpatrick M, Wytch R, Cole G, Helms P. Is the objective assessment of cerebral palsy gait reproducible? J Pediatr Orthop. 1994;14(6):705-708.

38. Zaino CA, Marchese VG, Westcott SL. Timed up and down stairs test: preliminary reliability and validity of a new measure of functional mobility. Pediatr Phys Ther. 2004;16(2):90-98.

39. Gordon AL, Ganesan V, Towell A, Kirkham FJ. Functional outcome following stroke in children. J Child Neurol. 2002;17(6):429-434.

40. McDonald CM, Henricson EK, Han JJ, et al. The 6-minute walk test as a new outcome measure in Duchenne muscular dystrophy. Muscle Nerve. 2010;41(4):500510.

41. Maher CA, Williams MT, Olds TS. The six-minute walk test for children with cerebral palsy. Int J Rehabil Res. 2008;31(2):185-188.

42. Verschuren $O$, Bosma L, Takken T. Reliability of a shuttle run test for children with cerebral palsy who are classified at Gross Motor Function Classification System level III. Dev Med Child Neurol. 2011;53(5):470-472.

43. Verschuren O, Takken T, Ketelaar M, Gorter JW, Helders PJ. Reliability for running tests for measuring agility and anaerobic muscle power in children and adolescents with cerebral palsy. Pediatr Phys Ther. 2007;19(2):108-115.

44. Verschuren O, Takken T, Ketelaar M, Gorter JW, Helders PJ. Reliability and validity of data for 2 newly developed shuttle run tests in children with cerebral palsy. Phys Ther. 2006;86(8):1107-1117. 
45. Caty GD, Arnould C, Thonnard JL, Lejeune TM. ABILOCO-Kids: a Rasch-built 10-item questionnaire for assessing locomotion ability in children with cerebral palsy. J Rehabil Med. 2008;40(10):823-830.

46. Sorsdahl AB, Moe-Nilssen R, Strand LI. Test-retest reliability of spatial and temporal gait parameters in children with cerebral palsy as measured by an electronic walkway. Gait Posture. 2008;27(1):43-50.

47. Wright FV, Ryan J, Brewer K. Reliability of the Community Balance and Mobility Scale (CB\&M) in high-functioning school-aged children and adolescents who have an acquired brain injury. Brain Inj. 2010;24(13-14):1585-1594.

48. McDowell BC, Humphreys L, Kerr C, Stevenson M. Test-retest reliability of a 1min walk test in children with bilateral spastic cerebral palsy (BSCP). Gait Posture. 2009;29(2):267-269.

49. Quinn A, O'Regan M, Horgan F. Psychometric evaluation of the functional walking test for children with cerebral palsy. Disabil Rehabil. 2011;33(25-26):23972403.

50. Wright FV, Liu G, Milne F. Reliability of the measurement of time-distance parameters of gait: a comparison in children with juvenile rheumatoid arthritis and children with cerebral palsy. Physiother. Canada. 1999;51(3):191-200.

51. Russell DJ, Palisano R, Walter S, et al. Evaluating motor function in children with Down syndrome: validity of the GMFM. Dev. Med. Child Neurol. 1998;40(10):693701.

52. lannaccone ST, Hynan LS. Reliability of 4 outcome measures in pediatric spinal muscular atrophy. Arch. Neurol. 2003;60(8):1130-6.

53. Russell DJ, Rosenbaum PL, Cadman DT, et al. The gross motor function measure: a means to evaluate the effects of physical therapy. Dev. Med. Child Neurol. 1989;31:341-352.

54. Bjornson K, Graubert C, McLaughlin J. Test-retest reliability of the gross motor function measure in children with cerebral palsy. Pediatr. Phys. Ther. 2000;12(4):200-2.

55. Kelly I.P. Jenkinson A., O'Brien T. OM. The quality assessment of walking in cerebral palsy. Gait Posture. 1997;5(1):70-74.

56. Malouin F, Richards CL, Menier C, Dumas F, Marcoux S. Supported Walker Ambulation Performance Scale (SWAPS): development of an outcome measure of locomotor status in children with cerebral palsy. Pediatr. Phys. Ther. 1997;9(2):4853.

57. Williams EN, Carroll SG, Reddihough DS, Phillips BA, Galea MP. Investigation of the timed "up \& go" test in children. Dev Med Child Neurol. 2005;47(8):518-524. 
58. Gan S-M, Tung L-C, Tang Y-H, Wang C-H. Psychometric properties of functional balance assessment in children with cerebral palsy. Neurorehabil. Neural Repair. 2008;22(6):745-53.

59. Van der Putten a, Vlaskamp C, Reynders K, Nakken H. Movement skill assessment in children with profound multiple disabilities: a psychometric analysis of the top down motor milestone test. Clin. Rehabil. 2005;19(6):635-643.

60. Tedla JS, Ganesan S, Katragadda S. Inter-rater reliability of the Top Down Motor Milestone Test: a cross-sectional study. Clin Rehabil. 2009;23(8):725-729.

61. Moody KD, Wright F V, Brewer KM, Geisler PE. Community mobility assessment for adolescents with an acquired brain injury: preliminary inter-rater reliability study. Dev Neurorehabil. 2007;10(3):205-211.

62. Novacheck TF, Stout JL, Tervo R. Reliability and validity of the Gillette Functional Assessment Questionnaire as an outcome measure in children with walking disabilities. J Pediatr Orthop. 2000;20(1):75-81.

63. Graham HK, Harvey A, Rodda J, Nattrass GR, Pirpiris M. The Functional Mobility Scale (FMS). J Pediatr Orthop. 2004;24(5):514-520.

64. Harvey AR, Morris ME, Graham HK, Wolfe R, Baker R. Reliability of the functional mobility scale for children with cerebral palsy. Phys Occup Ther Pediatr. 2010;30(2):139-149.

65. McDonald CM, Henricson EK, Han JJ, et al. The 6-minute walk test in Duchenne/Becker muscular dystrophy: longitudinal observations. Muscle Nerve. 2010;42(6):966-974.

66. Harvey A, Graham HK, Morris ME, Baker R, Wolfe R. The Functional Mobility Scale: ability to detect change following single event multilevel surgery. Dev Med Child Neurol. 2007;49(8):603-607.

67. Nordmark E, Jarnlo GB, Hägglund G. Comparison of the Gross Motor Function Measure and Paediatric Evaluation of Disability Inventory in assessing motor function in children undergoing selective dorsal rhizotomy. Dev. Med. Child Neurol. 2000;42(4):245-52.

68. Vos-Vromans DCWM, Ketelaar M, Gorter JW. Responsiveness of evaluative measures for children with cerebral palsy: the Gross Motor Function Measure and the Pediatric Evaluation of Disability Inventory. Disabil. Rehabil. 2005;27(20):124552.

69. Gan SM, Tung LC, Tang YH, Wang CH. Psychometric properties of functional balance assessment in children with cerebral palsy. Neurorehabil Neural Repair. 2008;22(6):745-753.

70. Cosmin Steering Committee. The COSMIN checklist. Available at: http://www.cosmin.nl/the-cosmin-checklist_8_5.html. Accessed May 23, 2013. 
71. De Vet HCW, Terwee CB, Knol DL, Bouter LM. When to use agreement versus reliability measures. J. Clin. Epidemiol. 2006;59(10):1033-9.

72. Mokkink LB, Terwee CB, Gibbons E, et al. Inter-rater agreement and reliability of the COSMIN (COnsensus-based Standards for the selection of health status Measurement Instruments) checklist. BMC Med. Res. Methodol. 2010;10(box C):82. 
Supplemental Table 2.1. Characteristics of the included measurement tools

\begin{tabular}{|c|c|c|c|c|c|c|c|c|c|c|c|c|c|c|c|c|c|c|c|c|}
\hline 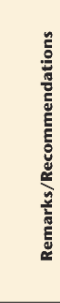 & 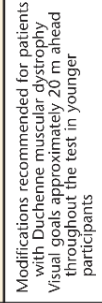 & 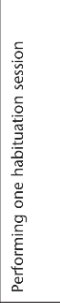 & 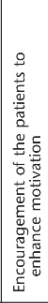 & 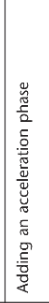 & 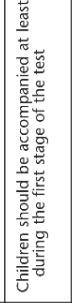 & 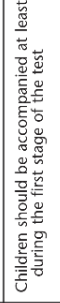 & 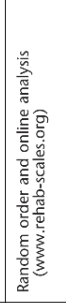 & 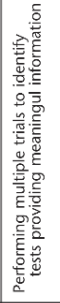 & & 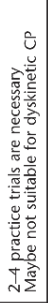 & & 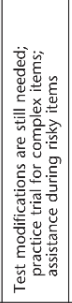 & 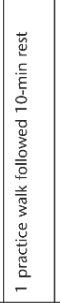 & & & & & & & 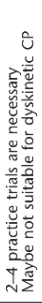 \\
\hline 薏 & & & & & & & & & & 产 & 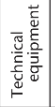 & & & & & & 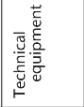 & 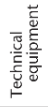 & & 产 \\
\hline 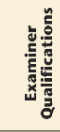 & 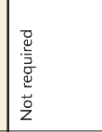 & 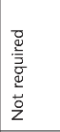 & 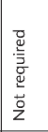 & 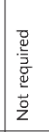 & 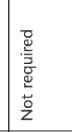 & 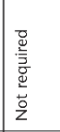 & 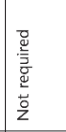 & 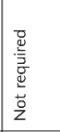 & & 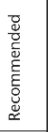 & 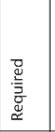 & 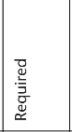 & 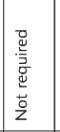 & 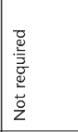 & 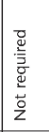 & & 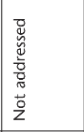 & 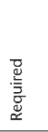 & 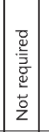 & 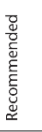 \\
\hline 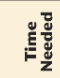 & $\circ$ & $\cong$ & n & in & i & i & in & 品 & & in & $:$ & o & in & in & $\because$ & & in & 8 & in & $n$ \\
\hline 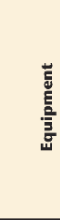 & 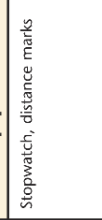 & 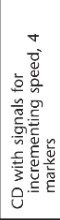 & 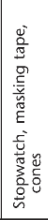 & 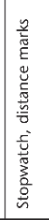 & 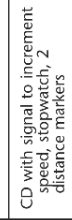 & 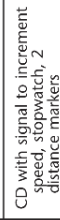 & & 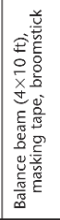 & & 产 & 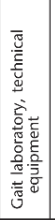 & 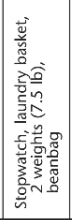 & 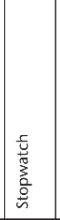 & 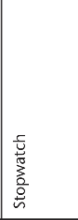 & 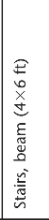 & & 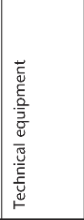 & 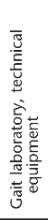 & 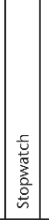 & 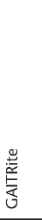 \\
\hline 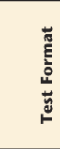 & 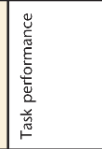 & 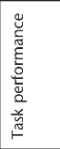 & 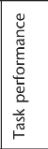 & 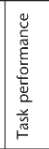 & 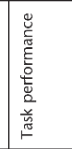 & 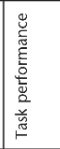 & 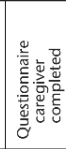 & 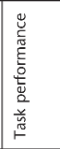 & & 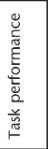 & 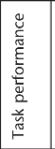 & 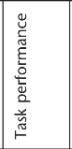 & 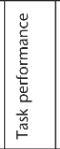 & 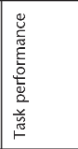 & 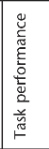 & & 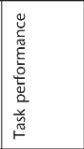 & 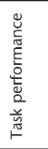 & 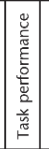 & 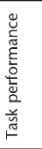 \\
\hline 莡 & 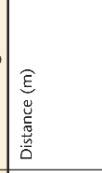 & 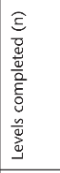 & 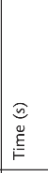 & 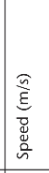 & 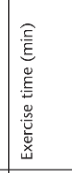 & 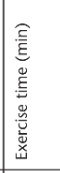 & 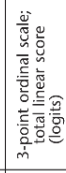 & 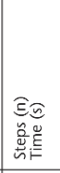 & & 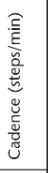 & 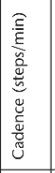 & 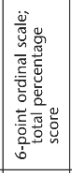 & 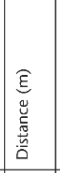 & 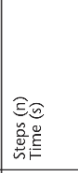 & 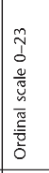 & & 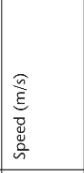 & 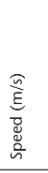 & 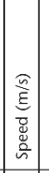 & 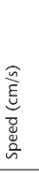 \\
\hline 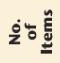 & & & & & & & & & & & & & & & & & & & & \\
\hline t. & 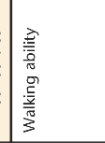 & 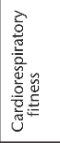 & 言 & 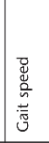 & 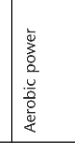 & 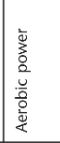 & 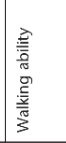 & 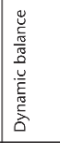 & & 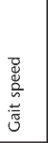 & \begin{tabular}{|l} 
总 \\
$\frac{2}{2}$ \\
$\frac{2}{\bar{v}}$ \\
\end{tabular} & 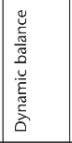 & 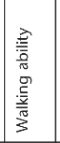 & 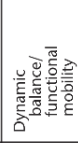 & 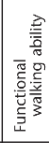 & & 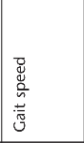 & 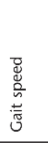 & 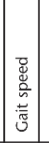 & 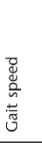 \\
\hline 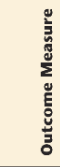 & 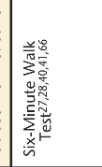 & 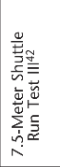 & 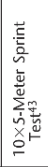 & 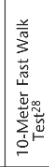 & 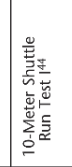 & 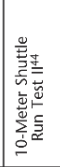 & 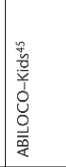 & 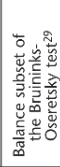 & 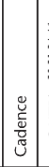 & 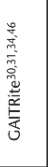 & 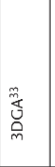 & 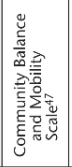 & 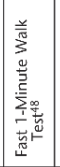 & 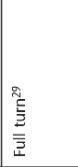 & 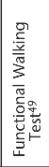 & 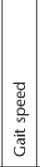 & 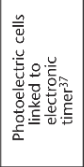 & $\begin{array}{l}\text { 第 } \\
\text { 峞 }\end{array}$ & 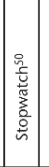 & 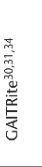 \\
\hline 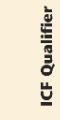 & $\mid \begin{array}{l}\text { 密 } \\
\text { 鄫 }\end{array}$ & & & & & & & & & & & & & & & & & & & \\
\hline
\end{tabular}


Supplemental Table 2.1. Continued

\begin{tabular}{|c|c|c|c|c|c|c|c|c|c|c|c|c|c|c|}
\hline 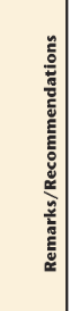 & 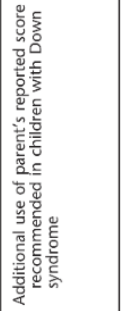 & & & & & 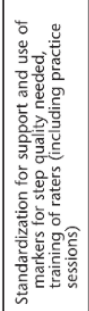 & & 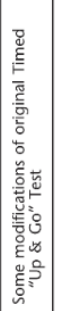 & & & & 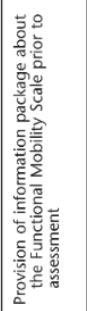 & 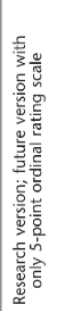 & 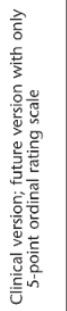 \\
\hline 仓ै & & 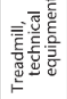 & & & & & & & $\frac{2}{n}$ & & & & n. & r. \\
\hline 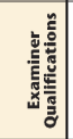 & 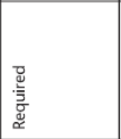 & 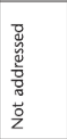 & 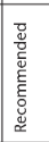 & 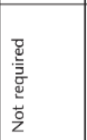 & 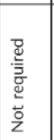 & 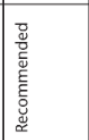 & 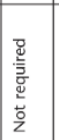 & 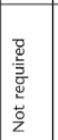 & 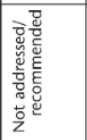 & 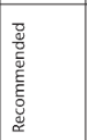 & 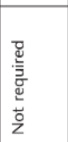 & 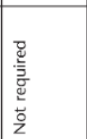 & 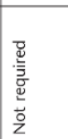 & 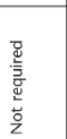 \\
\hline 㹂 & ㄱ. & $\sim$ & $\cong$ & in & $n$ & in & in & in & q & $\stackrel{m}{m}$ & in & in & $\stackrel{n}{2}$ & $\approx$ \\
\hline 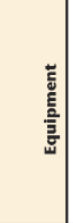 & 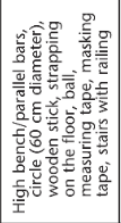 & 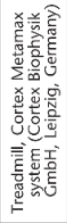 & 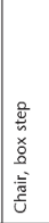 & & & & 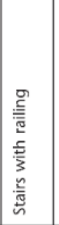 & 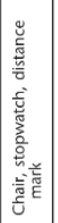 & & 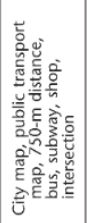 & & & & \\
\hline 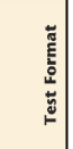 & 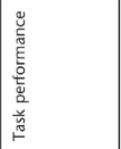 & 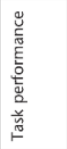 & 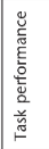 & 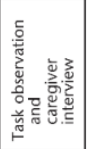 & 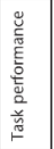 & 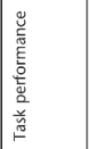 & 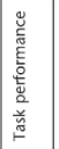 & 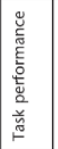 & 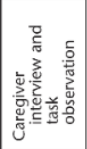 & 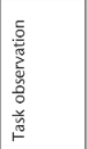 & 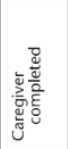 & 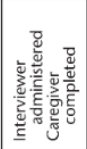 & 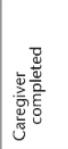 & 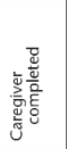 \\
\hline 竞 & 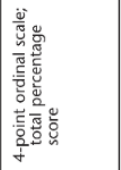 & 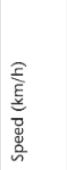 & 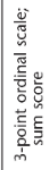 & 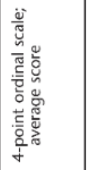 & 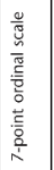 & 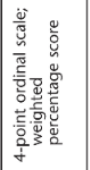 & $\begin{array}{l}\widehat{\widehat{\Xi}} \\
\tilde{\xi} \\
\underline{F}\end{array}$ & 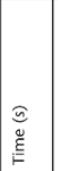 & 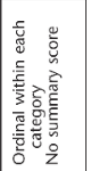 & 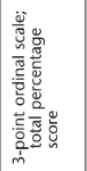 & 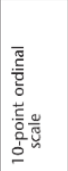 & 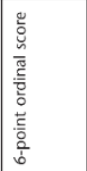 & 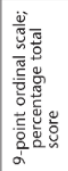 & 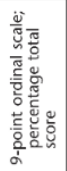 \\
\hline$\dot{\rho} z \underline{\underline{\underline{E}}}$ & $\stackrel{\sim}{\sim}$ & & $=$ & + & - & $\theta$ & & & 过 & & & $m$ & $\stackrel{\infty}{\sim}$ & f \\
\hline 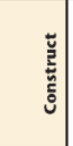 & 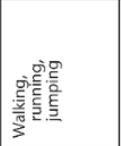 & 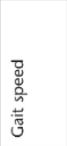 & 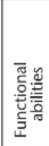 & 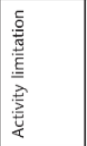 & $\begin{array}{l}\frac{2}{\frac{2}{2}} \\
\frac{5}{\bar{y}} \\
\frac{5}{5} \\
\frac{5}{15} \\
\frac{15}{3}\end{array}$ & 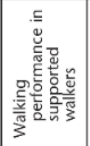 & 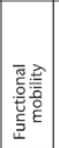 & 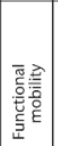 & 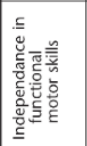 & 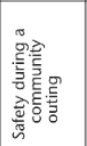 & 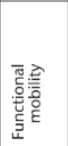 & 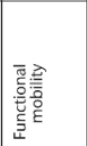 & 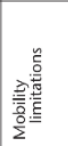 & 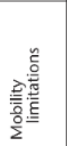 \\
\hline 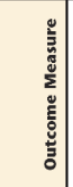 & 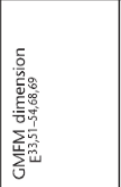 & 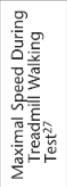 & 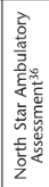 & 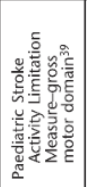 & 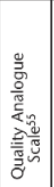 & 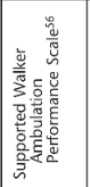 & 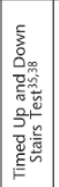 & 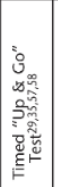 & 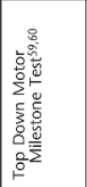 & 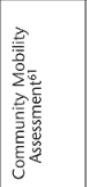 & 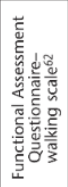 & 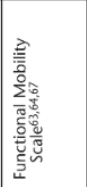 & 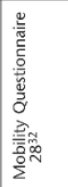 & 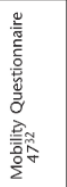 \\
\hline 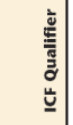 & 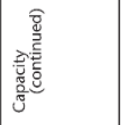 & & & & & & & & & 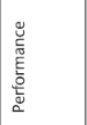 & & & & \\
\hline
\end{tabular}




\section{Supplemental Table 2.2. Search strategy}

\section{MEDLINE}

(c"gait" [MeSH] OR gait[tiab] OR gait/ataxia[tiab] OR gait/balance[tiab] OR gait/postural[tiab] OR gait/posture[tiab] OR gait'[tiab] OR gait's[tiab] OR gaitrite[tiab] OR gaitritewalkway[tiab] OR “walking" [MeSH] OR walk[tiab] OR walk/cycle[tiab] OR walk/go[tiab] OR walk/walk[tiab] OR walk'n[tiab] OR walk's[tiab] OR walkability[tiab] OR walkability' [tiab] OR walkable OR walked OR walker OR walker's OR walking[tiab] OR walking/activity[tiab] OR walking/balance[tiab] OR walking/gait[tiab] OR walking/mobility[tiab] OR walking/obstacles[tiab] OR walking' [tiab] OR walking's[tiab] OR "locomotion" [MeSH] OR mobility[tiab] OR mobility/activities[tiab] OR mobility/activity[tiab] OR mobility/adl[tiab] OR mobility/advancement[tiab] OR mobility/ambulation[tiab] OR mobility/ balance[tiab] OR mobility/community[tiab] OR mobility/disability[tiab] OR mobility/everyday[tiab] OR mobility/ independence[tiab] OR mobility/locomotion[tiab] OR mobility/walking[tiab] OR mobility'[tiab]) AND ("infant" [MeSH] OR infant*[tiab] OR infancy[tiab] OR "child"[MeSH] OR child*[tiab] OR schoolchild*[tiab] OR school age*[tiab] OR preschool*[tiab] OR kid[tiab] OR kids[tiab] OR toddler*[tiab] OR "adolescent" [MeSH] OR adoles"[tiab] OR teen*[tiab] OR boy*[tiab] OR girl"[tiab] OR "minors"[MeSH] OR minors*[tiab] OR "puberty" [MeSH] OR pubert*[tiab] OR pubescen*[tiab] OR prepubescen*[tiab] OR "pediatrics"[MeSH] OR paediatric*[tiab] OR peadiatric* [tiab] OR pediatric* [tiab] OR "schools" [MeSH] OR nursery school" [tiab] OR kindergar* [tiab] OR primary school" $\left[\right.$ tiab] OR secondary school* ${ }^{*}[$ tiab] OR elementary school"[tiab] OR high school" [tiab] OR highschool"[tiab]) AND ("Nervous System Diseases" [MeSH] OR "developmental disabilities" [MeSH] OR neuromuscular disease* [tiab] OR cerebral pals*[tiab] OR brain injur* [tiab] OR stroke[tiab] OR myelomeningocel* ${ }^{*}[$ tiab] OR meningomyelocel* [tiab] OR spinal cord injur*[tiab]) AND (instrumentation[sh] OR methods[sh] OR Validation Studies[pt] OR Comparative Study[pt] OR "psychometrics" [MeSH] OR psychometr"[tiab] OR clinimetr*[tw] OR clinometr* [tw] OR "outcome assessment (health care)" [MeSH] OR outcome assessment[tiab] OR outcome measure*[tw] OR "observer variation "[MeSH] OR observer variation[tiab] OR "Health Status Indicators" [MeSH] OR "reproducibility of results" [MeSH] OR reproducib*[tiab] OR "discriminant analysis"[MeSH] OR reliab*[tiab] OR unreliab"[tiab] OR valid"[tiab] OR coefficient[tiab] OR homogeneity[tiab] OR homogeneous[tiab] OR "internal consistency" [tiab] OR (cronbach"[tiab] AND (alpha[tiab] OR alphas[tiab])) OR (item[tiab] AND (correlation*[tiab] OR selection*[tiab] OR reduction*[tiab])) OR agreement [tiab] OR precision [tiab] OR imprecision[tiab] OR "precise values" [tiab] OR test-retest[tiab] OR (test[tiab] AND retest[tiab]) OR (reliab* [tiab] AND (test[tiab] OR retest[tiab])) OR stability[tiab] OR interrater[tiab] OR inter-rater[tiab] OR intrarater[tiab] OR intra-rater[tiab] OR intertester[tiab] OR inter-tester[tiab] OR intratester[tiab] OR intra-tester[tiab] OR interobserver[tiab] OR inter-observer[tiab] OR intraobserver[tiab] OR intra-observer[tiab] OR intertechnician[tiab] OR inter-technician[tiab] OR intratechnician[tiab] OR intra-technician[tiab] OR interexaminer[tiab] OR inter-examiner[tiab] OR intraexaminer[tiab] OR intra-examiner[tiab] OR interassay[tiab] OR interassay[tiab] OR intraassay[tiab] OR intra-assay[tiab] OR interindividual[tiab] OR inter-individual[tiab] OR intraindividual[tiab] OR intra-individual[tiab] OR interparticipant [tiab] OR inter-participant[tiab] OR intraparticipant[tiab] OR intra-participant[tiab] OR kappa[tiab] OR kappa's[tiab] OR kappas[tiab] OR repeatab*[tiab] OR ((replicab*[tiab] OR repeated[tiab]) AND (measure[tiab] OR measures[tiab] OR findings[tiab] OR result[tiab] OR results[tiab] OR test[tiab] OR tests[tiab])) OR generaliza*[tiab] OR generalisa*[tiab] OR concordance[tiab] OR (intraclass[tiab] AND correlation*[tiab]) OR discriminative[tiab] OR "known group" [tiab] OR factor analysis[tiab] OR factor analyses[tiab] OR dimension" [tiab] OR subscale" $[$ tiab] OR (multitrait[tiab] AND scaling[tiab] AND (analysis[tiab] OR analyses [tiab])) OR item discriminant[tiab] OR interscale correlation*[tiab] OR error[tiab] OR errors[tiab] OR "individual variability" [tiab] OR (variability[tiab] AND (analysis[tiab] OR values[tiab])) OR (uncertainty[tiab] AND (measurement[tiab] OR measuring[tiab])) OR "standard error of measurement" [tiab] OR sensitiv* [tiab] OR responsive*[tiab] OR ((minimal[tiab] OR minimally[tiab] OR clinical[tiab] OR clinically[tiab]) AND (important[tiab] OR significant [tiab] OR detectable[tiab]) AND (change[tiab] OR difference[tiab])) OR (small* [tiab] AND (real[tiab] OR detectable [tiab]) AND (change[tiab] OR difference[tiab])) OR "meaningful change" [tiab] OR "ceiling effect"[tiab] OR "floor effect" [tiab] OR "Item response model"[tiab] OR IRT[tiab] OR Rasch[tiab] OR "Differential item functioning" [tiab] OR DIF[tiab] OR "computer adaptive testing"[tiab] OR "item bank" [tiab] OR "cross-cultural equivalence"[tiab])) NOT (("addresses"[Publication Type] OR "biography"[Publication Type] OR "case reports"[Publication Type] OR "comment" [Publication Type] OR "directory" [Publication Type] OR "editorial"[Publication Type] OR "festschrift" [Publication Type] OR "interview"[Publication Type] OR "lectures"[Publication Type] OR "legal cases"[Publication 


\section{Supplemental Table 2.2. Continued}

Type] OR "legislation" [Publication Type] OR "letter" [Publication Type] OR "news" [Publication Type] OR "newspaper article" [Publication Type] OR "patient education handout" [Publication Type] OR "popular works" [Publication Type] OR "congresses" [Publication Type] OR "consensus development conference" [Publication Type] OR "consensus development conference, nih" [Publication Type] OR "practice guideline"[Publication Type] OR "adult" [MeSH] OR "Aged"[MeSH] OR "Aged, 80 and over" [MeSH]) OR ("animals" [MeSH Terms] NOT "humans" [MeSH Terms]))

Limits: only items with abstracts, humans, language: English, German

\section{CINAHL}

((MH "Walking+") OR gait" OR walk* OR mobility) AND ((MH "infant+") OR infant* OR infancy OR (MH "child+") OR child" OR schoolchild" OR school age" OR preschool" OR kid OR kids OR toddler" OR (MH "adolescence+") OR adoles $^{*}$ OR teen" OR boy* OR girl* OR (MH "Minors (Legal)") OR minors* OR (MH "puberty+") OR pubert* OR

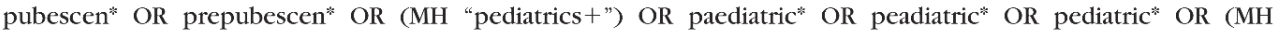
"schools+") OR nursery school* OR kindergar" OR primary school* OR secondary school* OR elementary school* OR high school* OR highschool") AND ((MH "Nervous System Diseases+") OR (MH "Developmental Disabilities") OR (MH "Child Development Disorders+") OR neuromuscular disease* OR cerebral pals* OR brain injur* OR stroke OR myelomeningocel* OR meningomyelocel* OR spinal cord injur*) AND ((MH "Research Measurement+") OR (MH "Outcomes Research") OR instrumentation* OR methods OR validation stud* OR comparative stud* OR psychometr* OR clinimetr* OR clinometr" OR (MH "Outcomes (Health Care)+") OR (MH "Treatment Outcomes+") OR (MH "Outcome Assessment") OR outcome assessment OR outcome measure* OR observer variation OR (MH "Health Status Indicators") OR (MH "reproducibility of results") OR reproducib* OR (MH "discriminant analysis") OR reliab" OR unreliab* OR valid" OR coefficient OR homogeneity OR homogeneous OR "internal consistency" OR (cronbach* AND (alpha OR alphas)) OR (item AND (correlation* OR selection* OR reduction*)) OR agreement OR precision OR imprecision OR "precise values" OR test-retest OR (test AND retest) OR (reliab* AND (test OR retest)) OR stability OR interrater OR inter-rater OR intrarater OR intra-rater OR intertester OR inter-tester OR intratester OR intra-tester OR interobserver OR inter-observer OR intraobserver OR intra-observer OR intertechnician OR inter-technician OR intratechnician OR intra-technician OR interexaminer OR inter-examiner OR intraexaminer OR intra-examiner OR interassay $O R$ inter-assay $O R$ intraassay $O R$ intra-assay $O R$ interindividual $O R$ inter-individual $O R$ intraindividual $O R$ intra-individual $O R$ interparticipant $O R$ inter-participant $O R$ intraparticipant $O R$ intra-participant $O R$ kappa $O R$ kappa's OR kappas OR repeatab* OR ((replicab* OR repeated) AND (measure OR measures OR findings OR result OR results OR test OR tests)) OR generaliza* OR generalisa* OR concordance OR (intraclass AND correlation*) OR discriminative OR "known group" OR factor analysis OR factor analyses OR dimension* OR subscale* OR (multitrait AND scaling AND (analysis OR analyses)) OR item discriminant OR interscale correlation* OR error OR errors OR "individual variability" OR (variability AND (analysis OR values)) OR (uncertainty AND (measurement OR measuring)) OR "standard error of measurement" OR sensitiv" OR responsive* OR ((minimal OR minimally OR clinical OR clinically) AND (important OR significant OR detectable) AND (change OR difference)) OR (small" AND (real OR detectable) AND (change OR difference)) OR "meaningful change" OR "ceiling effect" OR "floor effect" OR "Item response model" OR IRT OR Rasch OR "Differential item functioning" OR "computer adaptive testing" OR "item bank" OR "cross-cultural equivalence") NOT (MH "Adult+")

Limiters: abstract available; peer reviewed; exclude MEDLINE records; humans; language: English, German

\section{EMBASE}

(('walking'/exp OR gait* OR walk* OR 'physical mobility'/exp OR 'patient mobility'/exp) AND ('child'/exp OR 'adolescent'/exp OR infant*:ab,ti OR infancy:ab,ti OR child":ab,ti OR schoolchild*'ab,ti OR (school AND age*:ab,ti) OR preschool*:ab,ti OR kid:ab,ti OR kids:ab,ti OR toddler*:ab,ti OR adoles*:ab,ti OR teen*:ab,ti OR boy*ab,ti OR

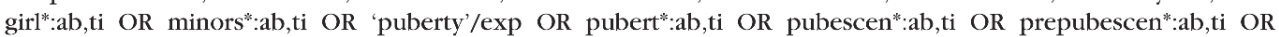




\section{Supplemental Table 2.2. Continued}

"pediatrics'/exp OR paediatric":ab,ti OR peadiatric":ab,ti OR pediatric":ab,ti OR 'school'/exp OR (nursery AND

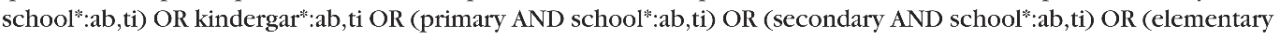
AND school*:ab,ti) OR (high AND school*:ab,ti) OR highschool":ab,ti) AND ('neurologic disease'/exp OR 'develop mental disorder'/exp OR (neuromuscular AND disease*:ab,ti) OR (cerebral AND pals*:ab,ti) OR (brain AND injur*: ab,ti) OR stroke:ab,ti OR myelomeningocel*:ab,ti OR meningomyelocel*:ab,ti OR (spinal AND cord AND injur*:ab,ti)) AND ('validation study'/exp OR psychometr" OR clin?metr" OR 'outcome assessment'/exp OR 'named inventories, questionnaires and rating scales'/exp OR "questionnaire'/exp OR "psychometry'/exp OR 'validity'/exp OR 'disability'/exp OR "reliability'/exp OR (outcome AND assessment":ab,ti) OR 'comparative study'/de OR (outcome AND measure":ab,ti) OR 'observer variation'/exp OR 'observer variation':ab,ti OR 'reproducibility'/exp OR reproducib”: ab,ti OR 'discriminant analysis'/de OR reliab":ab,ti OR unreliab":ab,ti OR valid":ab,ti OR coefficient:ab,ti OR homogeneity:ab,ti OR homogeneous:ab,ti OR 'internal consistency':ab,ti OR (cronbach*:ab,ti AND (alpha:ab,ti OR alphas: $a b, t i)$ ) OR (item:ab,ti AND (correlation*:ab,ti OR selection*:ab,ti OR reduction*:ab,ti)) OR agreement:ab,ti OR precision:ab,ti OR imprecision:ab,ti OR 'precise values':ab,ti OR 'test retest':ab,ti OR (test:ab,ti AND retest:ab,ti) OR (reliab*:ab,ti AND (test:ab,ti OR retest:ab,ti)) OR stability:ab,ti OR interrater:ab,ti OR 'inter rater':ab,ti OR intrarater: ab,ti OR 'intra rater':ab,ti OR intertester:ab,ti OR 'inter tester':ab,ti OR intratester:ab,ti OR 'intra tester':ab,ti OR interobserver:ab,ti OR 'inter observer':ab,ti OR intraobserver:ab,ti OR 'intra observer':ab,ti OR intertechnician:ab,ti OR 'inter technician':ab, ti OR intratechnician:ab,ti OR 'intra technician':ab,ti OR interexaminer:ab,ti OR 'interexaminer':ab,ti OR intraexaminer:ab,ti OR 'intra examiner':ab,ti OR interassay:ab,ti OR 'inter assay':ab,ti OR intraassay:ab,ti OR 'intra assay':ab,ti OR interindividual:ab,ti OR 'inter individual':ab,ti OR intraindividual:ab,ti OR 'intraindividual':ab,ti OR interparticipant:ab,ti OR 'inter participant':ab,ti OR intraparticipant:ab,ti OR 'intra participant': ab,ti OR kappa:ab,ti OR kappa*:ab,ti OR kappas:ab,ti OR repeatab*:ab,ti OR (replicab*:ab,ti OR repeated:ab,ti AND (measure:ab,ti OR measures:ab,ti OR findings:ab,ti OR result:ab,ti OR results:ab,ti OR test:ab,ti OR tests:ab,ti)) OR

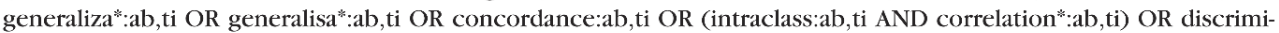
native:ab,ti OR 'known group':ab,ti OR (factor AND analysis:ab,ti) OR (factor AND analyses:ab,ti) OR dimension”: ab,ti OR subscale*:ab,ti OR (multitrait:ab,ti AND scaling:ab,ti AND (analysis:ab,ti OR analyses:ab,ti)) OR (item AND discriminant:ab,ti) OR (interscale AND correlation*:ab,ti) OR error:ab,ti OR errors:ab,ti OR 'individual variability': ab,ti OR (variability:ab,ti AND (analysis:ab,ti OR values:ab,ti)) OR (uncertainty:ab,ti AND (measurement:ab,ti OR measuring:ab,ti)) OR 'standard error of measurement':ab,ti OR sensitiv":ab,ti OR responsive*:ab,ti OR (minimal:ab,ti OR minimally:ab,ti OR clinical:ab,ti OR clinically:ab,ti AND (important:ab,ti OR significant:ab,ti OR detectable:ab,ti) AND (change:ab,ti OR difference:ab,ti)) OR (small*:ab,ti AND (real:ab,ti OR detectable:ab,ti) AND (change:ab,ti OR difference:ab,ti)) OR (meaningful AND change:ab,ti) OR 'ceiling effect':ab,ti OR 'floor effect':ab,ti OR 'item response model':ab,ti OR irt:ab,ti OR rasch:ab,ti OR 'differential item functioning':ab,ti OR 'computer adaptive testing':ab,ti OR 'item bank' ab,ti OR 'cross-cultural equivalence':ab,ti)) NOT 'animal'/exp NOT 'adult'/exp AND ([article]/lim OR [article in press]/lim OR [review]/lim) AND ([english]/lim OR [german]/lim) AND [humans]/lim AND [abstracts]/lim AND [embase]/lim NOT [medline]/lim

\section{PsycINFO}

((exp walking/ OR gait\$.mp. OR walk\$.mp OR exp physical mobility/) AND (child\$.mp. OR childhood.mp. OR adolescent.mp. OR infant\$.mp. OR infancy.mp. OR schoolchild\$.mp. OR (school and age).mp. OR preschool\$.mp. OR kid.mp. OR kids.mp. OR toddler\$.mp. OR adoles\$.mp. OR teen\$.mp. OR boy \$.mp. OR girl\$.mp. OR minors\$.mp OR exp puberty/OR pubert\$.mp. OR pubescen\$.mp. OR prepubescent\$.mp. OR exp pediatrics/ OR p? ediatric\$.mp. OR exp schools/ OR (nursery AND school\$).mp. OR kindergar\$.mp. OR (primary AND school\$).mp. OR (secondary AND school\$).mp. OR (elementary AND school\$).mp. OR (high AND school\$).mp. OR highschool\$.mp.) AND (exp nervous system disorders/ OR (neurologic AND disease\$).mp OR (neuromuscular AND disease\$).mp. OR (cerebral AND pals\$).mp. OR (brain AND injur\$).mp. OR stroke.mp. OR myelomeningocel\$.mp. OR meningomyelocel\$.mp. OR (spinal AND cord AND injur\$).mp.) AND (exp measurement/ OR exp test construction/ OR exp interrater reliability/ OR exp statistical analysis/ OR exp test reliability/ OR exp test validity/ OR (validation AND study).mp. OR psychometr\$.mp. OR clin?metr\$.mp. OR (outcome AND assessment\$).mp. OR (comparative AND study).mp. OR 


\section{Supplemental Table 2.2. Continued}

(outcome AND measure).mp. OR (observer AND variation).mp. OR reproducib\$.mp. OR (discriminant AND analysis).mp. OR reliab\$.mp. OR unreliab\$.mp. OR valid\$.mp. OR coefficient.mp. OR homogeneity.mp. OR homogeneous.mp. OR internal consistency.mp. OR (cronbach\$ AND (alpha OR alphas)).mp. OR (item AND (correlation\$ OR selection\$ OR reduction\$)).mp. OR agreement.mp. OR precision.mp. OR imprecision.mp. OR (precise AND value\$).mp. OR (test AND retest).mp. OR (reliab\$AND (test OR retest)).mp. OR stability.mp. OR interrater.mp. OR (inter AND rater).mp. OR intrarater.mp. OR (intra AND rater).mp. OR intertester.mp. OR (inter AND tester).mp. OR intratester.mp. OR (intra AND tester).mp. OR interobserver.mp. OR (inter AND observer).mp. OR intraobserver.mp. OR (intra AND observer).mp. OR intertechnician.mp. OR (inter AND technician).mp. OR intratechnician.mp. OR (intra AND technician).mp. OR interexaminer.mp. OR (inter AND examiner).mp. OR intraexaminer.mp. OR (intra AND examiner).mp. OR interassay.mp. OR (inter AND assay).mp. OR intraassay.mp. OR (intra AND assay).mp. OR interindividual.mp. OR (inter AND individual).mp. OR intraindividual.mp. OR (intra AND individual).mp. OR interparticipant.mp. OR (inter AND participant).mp. OR intraparticipant.mp. OR (intra AND participant).mp. OR kappa.mp. OR kappa\$.mp. OR repeatab\$.mp. OR (replicab\$ OR repeated AND (measure? OR findings OR result OR results OR test OR tests)).mp. OR generali?a\$.mp. OR concordance.mp. OR (intraclass AND correlation\$).mp. OR discriminative.mp. OR (known AND group).mp. OR (factor AND analys?s).mp. OR dimension\$.mp. OR sub scale\$.mp. OR (multitrait AND scaling AND analys?s).mp. OR (item AND discriminant).mp. OR (interscale AND correlation\$).mp. OR error.mp. OR errors.mp. OR (individual AND variability).mp. OR (variability AND (analys?s OR values)).mp. OR (uncertainty AND (measurement OR measuring)).mp. OR (standard AND error AND measure ment).mp. OR sensitive\$.mp. OR responsive\$.mp. OR (minimal OR minimally OR clinical OR clinically AND (important OR significant OR detectable) AND (change OR difference)).mp. OR (small\$ AND (real OR detectable) AND (change OR difference)).mp. OR (meaningful AND change).mp. OR (ceiling AND effect).mp. OR (floor AND effect).mp. OR (item AND response AND model).mp. OR irt.mp. OR rasch.mp. OR (differential AND item AND functioning).mp. OR (computer AND adaptive AND testing).mp. OR (item AND bank).mp. OR (cross AND cultural AND equivalence).mp.) 

CHAPTER 3

\section{CONCURRENT VALIDITY OF TWO GAIT PERFORMANCE MEASURES IN CHILDREN WITH NEUROMOTOR DISORDERS}
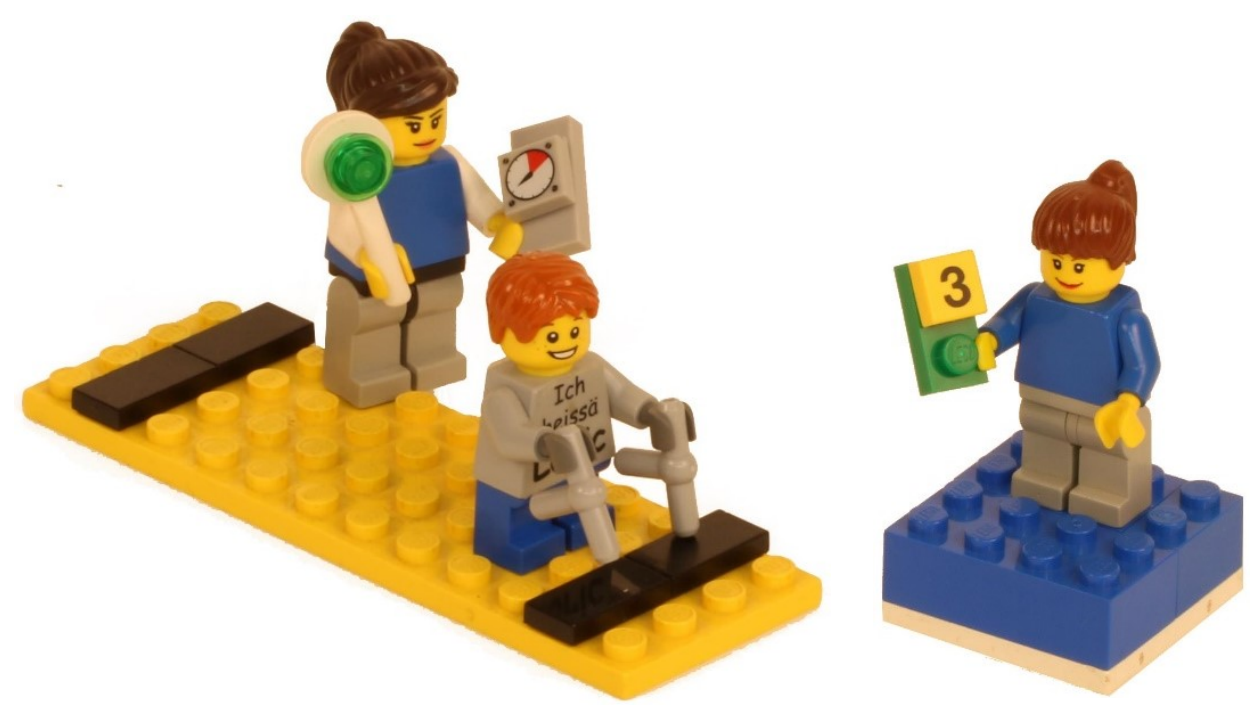

This chapter has been published as:

Ammann-Reiffer C, Bastiaenen CHG, Klöti C, van Hedel HJA. Concurrent validity of two gait performance measures in children with neuromotor disorders. Phys Occup Ther Pediatr. 2018; 2018 Jan 16:1-12. [Epub ahead of print]. 


\section{ABSTRACT}

AIMS: To investigate the concurrent validity of two mobility performance measures, the Functional Mobility Scale (FMS) and the Gillette Functional Assessment Questionnaire - walking scale (FAQ) in an inpatient pediatric neurorehabilitation setting.

METHODS: Cross-sectional data were collected on 71 children (mean age 12.7 years) with neuromotor gait impairments who participated in an inpatient rehabilitation program to evaluate aspects of concurrent validity of the FMS and FAQ. Physiotherapists independently performed ratings. Comparator measures included the walking item of the Functional Independence Measure for children, 10meter and 6-minute walk tests, and Gross Motor Function Measure-88 dimension E. All tests were completed within seven days. Spearman correlation coefficients were calculated to test a priori formulated hypotheses regarding the strength and direction of the measures' relationships.

RESULTS: The children had a broad spectrum of mobility levels, including all levels of FMS and levels 2-10 of FAQ. Spearman correlation coefficients with comparator measures varied between .58-.79 for the FMS and .69-.73 for the FAQ. Hypotheses concerning correlation strengths and directions were met for FMS and FAQ.

CONCLUSIONS: The study expands the existing evidence of the measures' validity and demonstrates that the FMS and FAQ are valid to evaluate functional mobility in pediatric inpatient neurorehabilitation. 


\section{INTRODUCTION}

Walking ability is often a primary focus in the treatment of children undergoing inand outpatient neurorehabilitation. According to the International Classification of Functioning, Disability, and Health for Children and Youth (ICF-CY), walking, as a category of mobility, can be either characterized as motor capacity or motor performance. ${ }^{1}$ While walking capacity reflects an individual's highest probable level of walking in a standardized environment, walking performance describes how an individual moves around in his or her current environment. ${ }^{2}$ Additionally, the construct motor capability characterizes a person's highest probable level of walking in his or her daily environment. ${ }^{2}$ Although related to each other, the evaluation of motor performance and capability provide insight into a child's activity level in daily life by taking into account the child's physical and social environment. Outcome measures considering these factors open new perspectives as the environmental setting has a substantial impact on children's and adolescents' type of mobility. ${ }^{3}$ Thus, measures of motor performance have their own value besides the outcomes of motor capacity measures when assessing walking abilities.

Despite the value of measures that assess walking performance or capability, gait therapy outcome is predominantly evaluated with tests reflecting body functions or walking capacity. One reason might be the shortage of measures for the assessment of gait performance. ${ }^{4}$ Furthermore, motor performance may seem harder to assess because it can differ depending on the environment to which it refers. Nevertheless, walking performance and capability are important constructs to measure because they provide an opportunity to incorporate new perspectives into treatment planning and goal setting that are meaningful for the child and the family in everyday life.

The Functional Mobility Scale (FMS) and the Functional Assessment Questionnaire - walking scale (FAQ) are two examples of performance measures to assess functional mobility. The FMS has been developed for children with cerebral palsy (CP) and describes the level of a child's functional mobility by assessing the assistive device that is used in everyday life over three different distances. ${ }^{5}$ Usually, a clinician administers the scale as a semi-structured interview with the child or a parent. 
Several studies evaluating the psychometric properties of the FMS in children with CP have shown very positive results. ${ }^{5-8}$

The FAQ encompasses a range of walking abilities on a 10 -level ordinal scale. ${ }^{9}$ The child or parent are asked to identify the child's usual level of functioning by reading the descriptions of the different levels. The FAQ has moderate to strong correlations with other mobility scales and fair correlations with several parameters measured during level-ground walking. ${ }^{9}$

To our knowledge, the original English versions of these measures have only been used in outpatient settings evaluating children's functioning in their home, community, and school settings. In a recent study, we had shown that interrater reliability of the German FMS and FAQ was substantial to almost perfect between parents, nurses, and physiotherapists (linear weighted kappa coefficients FMS-5: .62-.85; FMS-50: .79-.92; FMS-500: .83-.90; FAQ: .69-.77). Furthermore, ratings between parents, nurses, and physiotherapists did not differ significantly when assessed in the inpatient setting and the children's usual environment. ${ }^{10}$ The purpose of the present study was to investigate the concurrent validity of the German FMS and FAQ (see Appendix) within the framework of the ICF-CY in children with neuromotor disorders in an inpatient setting. We compared FMS and FAQ scores with other performance and capacity measures that are frequently used to assess walking ability in neuropediatric rehabilitation. To test the validity of the FMS/FAQ, we had formulated several hypotheses a priori:

(1) Correlations between the FMS and capacity measures of walking ability (6minute walk test (6MinWT), 10-meter walk test self-selected and maximal speed (10MWTss/m), Gross Motor Function Measure-88 dimension E (GMFM E)) as well as the walking item of the Functional Independence Measure for Children (WeeFIM) are positive and moderate to strong, with Spearman correlation coeffiecients $\left(r_{\mathrm{s}}\right) \geq .50$.

(2) Correlations between the FAQ and capacity measures of walking ability (6MinWT, 10MWTss, 10MWTm, GMFM E) as well as the WeeFIM walking item are positive and moderate to strong, with $r_{s} \geq .60$. 
(3) The order of correlation strengths of the FMS/FAQ is WeeFIM walking item $>$ timed walking tests (6MinWT/10MWTss/10MWTm) > GMFM E.

Based on the definitions by Chung, ${ }^{11}$ we expected moderate (.49-.59) to strong (.60.79) positive correlations. We expected higher correlations between the FMS/FAQ and the WeeFIM because the WeeFIM is a performance measure while the other comparator measures are capacity measures. As the FMS is the only measure that considers the type of walking aids used for functional mobility, we expected that the FMS correlated less $\left(r_{s}>.50\right)$ than the FAQ $\left(r_{s}>.60\right)$ with the comparator measures.

\section{METHODS}

\section{Design}

This was a cross-sectional study, nested within a larger longitudinal study that evaluated responsiveness as well as reliability aspects of the two measures. ${ }^{10}$ All measurements were performed at the Rehabilitation Center Affoltern am Albis of the University Children's Hospital Zurich, Switzerland. Our center provides in- and outpatient treatment for children and adolescents with predominantly acquired and congenital neurological diagnoses. The most common reasons for admission to our center are postsurgical treatment and improving independence in everyday life activities. The study was approved by the Ethics Committee Zurich and registered on ClinicalTrials.gov (NCT02237222).

\section{Participants}

Children were eligible for the study if they had a neuromotor disorder and were six to18 years old at admission. They had to be inpatients at the rehabilitation center with a treatment goal of acquiring, retaining or improving gait function. Therapists reported children who met these inclusion criteria. These children and their parents subsequently received oral and written study information by a research assistant and were consecutively recruited between April 2014 and January 2016, provided that a signed informed consent and assent by the legal guardian and the child had been given. Adhering to the criteria provided by the COSMIN guidelines, we aimed for a sample size of at least $50 .{ }^{12}$ 
The participants were 71 children and adolescents ( 26 females, 45 males) with a mean age of 12.7 years (SD 3.2y, range $6.0-18.6 y$ ). The majority of the children $(n=69)$ had also participated in an earlier reliability study. ${ }^{10}$

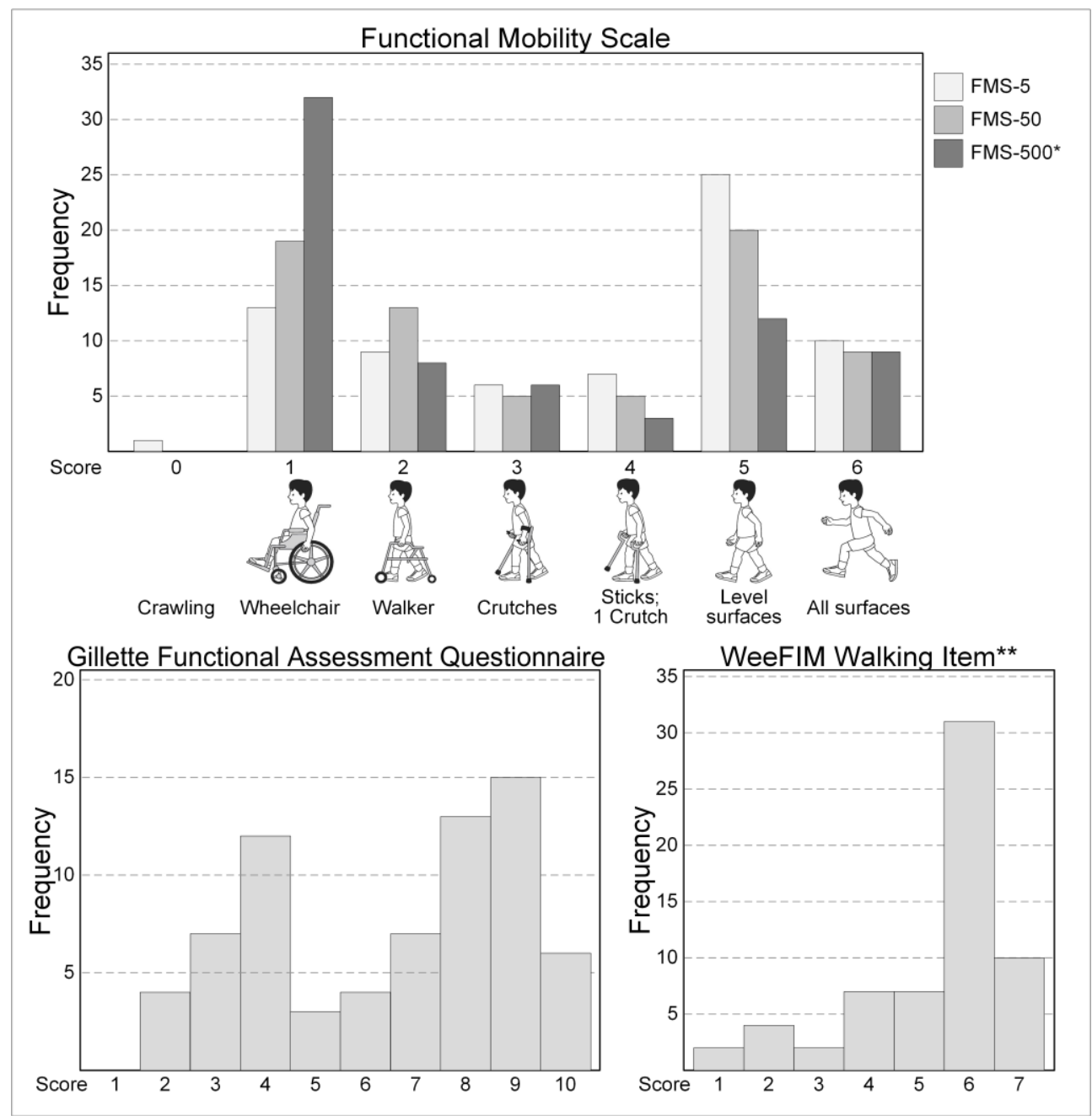

Figure 3.1. Participants' scores on the performance measures $(n=71)$.

n, number; FMS-5/50/500, Functional Mobility Scale over 5/50/500 meters; WeeFIM, Functional Independence Measure for Children; *, $\mathrm{n}=1$ missing; ${ }^{* *}, \mathrm{n}=63$ as 8 children were rated with respect to wheelchair mobility

CP was the most frequent diagnosis ( $n=38$; GMCS level I/II/II//V: 7/12/13/6), followed by traumatic brain injury $(n=10)$, genetic disorders $(n=7)$, stroke $(n=6)$, encephalitis $(n=3)$, and other comparable diagnoses $(n=7)$. Nineteen children mainly 
used a wheelchair when moving inside the rehabilitation center, 23 required a walking device, and 29 walked without any assistance and walking aids. Due to this diversity of functional abilities, not all children were able to perform all capacity tests. Details regarding the children's level of functional mobility and walking capacity are shown in Figure 3.1. and Table 3.1.

Table 3.1. Score distribution of the capacity measures

\begin{tabular}{lcccc}
\hline Measure; unit & $\mathrm{N}$ & Mean (SD) & Median (IQR) & Range \\
\hline 10MWTss; m/s & 58 & $.79(.40)$ & $.85(.70)$ & $.09-1.44$ \\
10MWTm; m/s & 50 & $1.26(.61)$ & $1.36(.80)$ & $.17-2.88$ \\
6MinWT; m & 54 & $299(179)$ & $307(344)$ & $38-634$ \\
GMFM E; $\%$ & 35 & $46.8(33.9)$ & $54(61)$ & $0-97.2$ \\
\hline
\end{tabular}

Abbreviations: $n$, number; SD, standard deviation; IQR, interquartile range; 10MWTss, 10-m walking test self-selected speed; 10MWTm, 10-m walking test maximal speed; 6MinWT, 6-minute walking test; GMFM E, Gross Motor Function Measure dimension $\mathrm{E}$

\section{Measures}

Index measures

Functional Mobility Scale (FMS). The FMS scores the assistive device that a child uses in everyday life over 5,50 , and 500 meters. ${ }^{5}$ These distances represent typical distances covered by children at home, in school, and the wider community. For each distance, the type of assistance required is rated on an ordinal scale from 1 (uses a wheelchair) to 6 (independent walking without an assistive device on all surfaces). The FMS has been compared with other measures of gait function in 310 ambulatory children with spastic cerebral palsy (CP). It has shown strong positive correlations with the physical function and sports domain of the Pediatric Outcomes Data Collection Instrument $\left(r_{s}=.89\right)$, the time spent in an upright position measured with a remote activity monitor $\left(r_{s}=.87\right)$, the physical functioning domain of the Child Health Questionnaire $\left(r_{s}=.78\right)$, and the Rancho Scale $\left(r_{s}=.78\right)$. Further, the FMS correlated moderately with the Energy Expenditure measured during a 10-minute walk at self-selected speed $\left(r_{s}=.51\right) .{ }^{5}$ FMS scores obtained by parent interview and direct observation in 4- to 18-year-old children with CP agreed substantially. ${ }^{7}$

Functional Assessment Questionnaire - walking scale (FAQ). The FAQ describes various levels of mobility, differing in environment, terrains or obstacles. The scale descriptions range from 1 (the child cannot take any steps at all) to 10 (the child 
walks, runs, and climbs on level and uneven terrain and does stairs without difficulty or assistance. Is typically able to keep up with peers). The child or parent is asked to identify the level that best describes the child's mobility level by taking into account the walking device that is typically used in daily life.

The content and concurrent validity of the FAQ have been assessed in comparison with other measures in 2- to 17-year-old children with neuromuscular diagnoses. The scale showed strong positive correlations with the transfers and basic mobility scale of the Pediatric Orthopaedic Society of North America ( $r=.76)$ and the gross motor subscale of the Functional Independence Measure for Children (WeeFIM; r=.64), and moderate positive correlations above .40 with the Normalcy Index and oxygen consumption during level-ground walking. ${ }^{9}$

\section{Comparator measures}

6-minute walk test (6MinWT). This test investigates functional capacity. ${ }^{13}$ Its testretest reliability in children with $\mathrm{CP}$ is high (intraclass correlation coefficient (ICC) $=.98$ ), and there is limited to moderate evidence for convergent validity with various parameters of the ICF-CY body function domain in children with chronic conditions. ${ }^{4,14}$

10-meter walk test. This is a measure of functional mobility that evaluates selfselected (10MWTss) or maximal (10MWTm) gait speed over a short distance. ${ }^{15}$ Relative test-retest reliability of the 10MWT is high (10MWTss: ICC=.90; 10MWTm: $\mathrm{ICC}=.81)$ in children with neurological gait disorders and $\mathrm{CP}$, respectively. The standard error of measurement varies between 1.85 seconds (10MWTm) and 3.61 seconds (10MWTss). ${ }^{4,16}$

Gross Motor Function Measure-88 dimension E (GMFM E). This is a standardized measure of gross motor function in children with motor disabilities. ${ }^{17}$ The 24 ordinalscale items of the dimension $E$ assess walking, running, and jumping abilities. The psychometric properties of the total GMFM-88 are well explored, especially in children with CP. ${ }^{18}$ Evidence for the psychometric properties of the single dimensions is unknown because the patient samples of the existing studies were too small. ${ }^{4}$ 
Because of logistical reasons, in our study, the GMFM E was only administered to children who performed a robotic-assisted treadmill training as part of their inpatient rehabilitation.

Walking item of the Functional Independence Measure for Children (WeeFIM). This measure assesses a child's level of independence during various activities of daily living on the performance level. ${ }^{19}$ The measure is comprised of 18 items scored on a 7-point ordinal scale, covering the domains Self-Care, Mobility, and Cognition. The WeeFIM has excellent test-retest and interrater reliability, and several studies of its validity and responsiveness have been published. For the locomotion subscale, intra- and interrater reliability is high (ICC: .94-.97 and .73-.85 respectively) and correlations with mobility domains of other measures of functional performance are strong. ${ }^{20-22}$

In our study, only the walking item of the WeeFIM locomotion subscale was scored, and only in those children who were expected to be walking at discharge.

\section{Translation procedure}

To facilitate the implementation of the two gait performance measures in the German-speaking clinical setting, we translated the original English versions into German by adhering to the guidelines of the American Association of Orthopaedic Surgeons Outcomes Committee:23 (1) independent German translation of the two questionnaires by two German-speaking health professionals; (2) combining these two translations into a synthesized version; (3) back-translation into English by a professional translation agency and a native English speaking person, both blinded for the original version and naïve on the construct to be measured; (4) obtaining approval of the back-translated consensus version by the original developers of the questionnaires (the corresponding author can be contacted for translated measures).

\section{Procedure}

Before scoring the FMS and the FAQ, physiotherapists $(n=15)$ responsible for providing treatment to the participating children received oral and written instructions regarding the use of the FMS and FAQ. They were blinded to other test results. The 
physiotherapists completed the questionnaires in a quiet room, in the absence of the children. To ensure that they took their time to think about the suitable rating, they were assisted by an independent research fellow, who was responsible that no missing values occurred. The research fellow performed a standardized interview to ensure that the raters scored the child's functional mobility in the inpatient setting on the performance level and adhered to the standardized instructions of the authors of the original English versions. Details of the implementation of the other tests are outlined in Table 3.2 .

Table 3.2. Details of the test procedures for the comparator measures.

\begin{tabular}{|c|c|c|c|}
\hline Measures & Test description & Equipment & Assessor \\
\hline $6 \mathrm{MinWT}$ & $\begin{array}{l}30 \text {-m track in a quiet corridor of the } \\
\text { rehabilitation center, } 2 \text { cones at each } \\
\text { end of the track } \\
\text { Walking as many meters as possible } \\
\text { within } 6 \text { minutes } \\
\text { Standardized instructions during the test } \\
\text { [9] } \\
\text { Covered distance recorded to } 1 \mathrm{~m}\end{array}$ & $\begin{array}{l}\text { Usual walking aids and } \\
\text { orthoses }\end{array}$ & Therapist* \\
\hline 10MWT & $\begin{array}{l}\text { 14-m track in a quiet corridor of the } \\
\text { rehabilitation center with marks at } 0 / \\
2 / 12 / 14 \mathrm{~m}\end{array}$ & $\begin{array}{l}\text { Usual walking aids and } \\
\text { orthoses }\end{array}$ & Therapist* \\
\hline 10MWTss & $\begin{array}{l}\text { Walking from the first to the last line in } \\
\text { comfortable speed }\end{array}$ & & \\
\hline 10MWTm & $\begin{array}{l}\text { Walking as fast as possible without } \\
\text { running } \\
\text { Time needed for the middle } 10 \mathrm{~m} \\
\text { Mean of } 2 \text { trials (10MWTss); faster out of } 2 \\
\text { trials (10MWTm) }\end{array}$ & & \\
\hline GMFM E & $\begin{array}{l}\text { Quiet room, corridor with test marks, } \\
\text { staircase with handrails at each side } \\
\text { Test instructions and scoring according to } \\
\text { GMFM-manual } \\
\text { Calculation of percentage-score }\end{array}$ & $\begin{array}{l}\text { Barefoot, without walking } \\
\text { aids }\end{array}$ & $\begin{array}{l}\text { Therapist with GMFM } \\
\text { certification* }\end{array}$ \\
\hline $\begin{array}{l}\text { WeeFIM } \\
\text { Walking }\end{array}$ & $\begin{array}{l}\text { Motor performance on the ward } \\
\text { Walking score only for children being } \\
\text { expected to walk at discharge }\end{array}$ & $\begin{array}{l}\text { Usual walking aids and } \\
\text { orthoses }\end{array}$ & $\begin{array}{l}\text { Nurse being responsible } \\
\text { for daily care* }\end{array}$ \\
\hline
\end{tabular}

Abbreviations: 6MinWT, 6-minute walking test; 10MWTss, 10-m walking test self-selected speed; 10MWTm, 10-m walking test maximal speed; GMFM E, Gross Motor Function Measure dimension E; WeeFIM Walking, Functional Independence Measure for Children walking item; *, blinded to the FMS/FAQ results

While the WeeFIM scores originated from data that were gathered as part of the clinical routine, all other data were collected for research purposes. The execution of the tests did not follow a predetermined order. 
All measurements were completed within seven days to ensure that the children's condition was stable. Age at first assessment, gender, diagnosis, and mobility level of each child was recorded.

\section{Statistical analysis}

Data were captured in Microsoft Office Access 2007 (Microsoft Corporation, Redmond, WA, USA) and analyzed using SPSS version 23.0 (IBM SPSS Statistics, Chicago, IL, USA). Descriptive parameters including gender, age, diagnosis, dependence on assistive mobility device, and FMS/FAQ scores described the study population.

To quantify the relationship between the FMS/FAQ and the comparator measures, we calculated $r_{s}$ and 95\% confidence intervals employing bootstrapping (a bias corrected and accelerated method) without replacement for missing data. We interpreted values from $0-.19$ as very weak, .20-.39 as weak, .40-.59 as moderate, $.60-.79$ as strong, and $\geq .80$ as very strong. ${ }^{11}$ Concerning the hypotheses 1 and 2 , we were more interested in the construct of "walking ability on the capacity level" rather than in each single capacity measure. Therefore, we decided in line with Peter et al. ${ }^{24}$ to calculate one correlation coefficient reflecting all capacity measures. To average these correlations adequately, we transformed the individual correlation coefficients of the capacity tests into Fisher's Z scores, summarized and averaged these scores, and transferred the averaged Fisher's $Z$ scores back into a correlation coefficient. ${ }^{24}$ The statistical level of significance $\alpha$ was set at 0.05 .

\section{RESULTS}

The FMS and the FAQ correlated with the comparator measures in a positive direction from .58-.79 and .69-.73, respectively (Table 3.3.). Correlation strengths with the WeeFIM walking item increased considerably from FMS-5 to FMS-500. No difference in correlation strength was observable between the three FMS-scores and the averaged capacity tests $\left(r_{s}=.73\right)$. Correlations between the FAQ and the comparator measures were all very similar and ranged between .68-.79. Correlations between the FAQ and the FMS were $.77, .86$, and .86 for the FMS-5,-50, and -500 , respectively. 
Table 3.3. Spearman's rank correlation coefficients ( $95 \%$ confidence intervals) for the scores on the Functional Mobility Scale and Gillette Functional Assessment Questionnaire - walking scale, and the scores obtained on other clinical measures

\begin{tabular}{lcccccc}
\hline & & 10MWTss & 10MWTm & 6 MinWT & GMFME & \multicolumn{2}{c}{ WeeFIM walking } \\
$\mathrm{N}$ & Averaged capacity tests & 58 & 50 & 54 & 35 & 63 \\
\hline FMS-5 & $.73(.58-.84)$ & $.68(.54-.78)$ & $.72(.55-.84)$ & $.73(.58-.83)$ & $.79(.63-.90)$ & $.58(.40-.72)$ \\
FMS-50 & $.73(.55-.85)$ & $.71(.56-.80)$ & $.75(.58-.86)$ & $.76(.62-.85)$ & $.69(.44-.88)$ & $.65(.49-.78)$ \\
FMS-500 & $.73(.55-.85)$ & $.73(.58-.83)$ & $.75(.58-.86)$ & $.76(.62-.85)$ & $.68(.42-.87)$ & $.79(.68-.86)$ \\
FAQ & $.71(.53-.85)$ & $.70(.54-.82)$ & $.71(.54-.84)$ & $.73(.57-.85)$ & $.69(.45-.87)$ & $.71(.59-.80)$ \\
\hline
\end{tabular}

$\mathrm{P}<0.01$ for all comparisons

Abbreviations: FMS-5/50/500, Functional Mobility Scale; FAQ, Gillette Functional Assessment Questionnaire; 10MWTss, 10-m walking test self-selected speed; 10MWTm, 10-m walking test maximal speed; 6MinWT, 6-minute walking test; GMFM E, Gross Motor Function Measure dimension E; WeeFIM walking, Functional Independence Measure for Children walking item

The results regarding our a priori hypotheses were as follows:

(1) Correlations of the FMS with the WeeFIM walking and the capacity tests were .58 and .73 , respectively, which confirmed hypothesis $1\left(r_{s} \geq .50\right)$.

(2) Correlations of the FAQ with the WeeFIM walking and the capacity tests were .71 , which confirmed hypothesis $2\left(r_{s} \geq .60\right)$.

(3) The hypothesized order of correlation strengths (WeeFIM walking > timed walking tests > GMFM E) was only for the FMS-500 as expected; hypothesis 3 was rejected.

\section{DISCUSSION}

This study aimed to evaluate the concurrent validity of the FMS and FAQ in an inpatient setting in children with neuromotor disorders within the framework of the ICF-CY. For this, we used comparator measures that are well known and often used to assess walking abilities in clinical practice as well as in clinical research. Two out of three of the hypotheses concerning the correlation strengths and directions were met for the FMS and the FAQ.

All correlation coefficients were moderate to strong and corresponded well to the correlation strengths reported in the original studies. ${ }^{5,9}$ This indicates an underlying common construct "walking ability". A further construct that is measured by the FMS/FAQ is "performance". However, only the FMS-500 showed the highest correlation coefficient with the other performance measure, the WeeFIM walking. For the $F A Q$, all correlations were comparable, while the magnitude of the correlations 
between FMS-5 and FMS-50 scores and WeeFIM walking scores were lower than with the capacity measures.

Whereas almost $50 \%$ of our children were mobile without using a walking aid over 5 meters, this amount decreased to $41 \%$ over 50 meters and $30 \%$ over 500 meters, while $45 \%$ of the children used a wheelchair for the distance of 500 meters. A similar shift in use of walking aids relative to walking distance or environmental setting was observed by Harvey et al., ${ }^{7}$ who examined the construct validity of the FMS by analyzing the agreement between FMS scores and direct observation of children's mobility around and outside their home and at school.

None of the correlation coefficients with the other measures exceeded .80, which is another indication that the FMS and FAQ add new information on functional mobility in the inpatient setting and have a complementary value in the assessment of children's walking abilities by offering further insights into the complex construct of functional mobility with very little additional effort.

While the floor and ceiling effects are a known issue with the mobility subscale of the WeeFIM, ${ }^{25,26}$ it does not seem to affect the FMS and FAQ. The FAQ bypasses the problem of possible floor effects, as the levels 1-4 of its scale address both walking capability and walking performance. This combination is of particular interest for children who require assistance or supervision for walking. Although these children would be able to walk, they usually have to depend on a wheelchair because, throughout the day, there is no or only limited assistance from another person available. This leads to a decrease in the performance rating, while the capability rating is not affected. On the other end of the FAQ-scale, walking abilities on various surfaces, in different environments, and using different types of locomotion are addressed, which prevents a ceiling effect in most children with neuromotor impairments. The FMS exclusively addresses functional mobility on the performance level. In our sample, $18 \%$ of the children were rated as primarily using a wheelchair in daily routine over all three distances of the FMS. This fact might hint at a possible presence of a floor effect. However, if the FMS is used to measure functional mobility regarding a child's walking ability, it should not be of interest whether it can distinguish further between these children. ${ }^{27}$ 


\section{Limitations}

The FMS and FAQ have been validated in outpatient settings evaluating the children's functional mobility in their home, school, and community setting. We used an inpatient setting with the rating of the children's inpatient performance for the evaluation of concurrent validity. This approach allowed us to not only validate the German versions but also gain some knowledge of psychometric properties in this setting. Our method of data collection for the FMS and FAQ differed from the original test administration by parent- or child-report. Although parents' or children's view would be most relevant in terms of performance, we necessarily chose to ask the therapists, because parents are only occasionally present in the rehabilitation center and children's cognitive abilities do not always allow a self-report. This is a limitation of the inpatient setting. With the standardized interviews, we were able to ensure that the therapists actually scored the FMS and FAQ on performance level and not on the capacity level, as capacity measures are more often used among therapists. This approach with therapists' scores and standardized interviews might have influenced the correlation strength between the FMS/FAQ and the comparator measures. We used only the dimension $E$ of the GMFM and the walking item of the WeeFIM instead of the complete measures, regardless of the missing evidence on their psychometric properties. Being aware of the limitations, we chose this approach, as we wanted to compare the FMS and FAQ to measures of walking ability only, and not to multidimensional instruments. Further, we did not randomize the order of test administration; however, we did not administer the measures in a fixed order either.

Previous studies showed that children with CP with similar levels of motor capacity or motor capability present with different levels of motor performance when walking in their own environment. ${ }^{2,28}$ However, our results might imply that there is no large difference between the level of walking performance and walking capacity. A contributing factor could be that children in our study were assessed during an inpatient neurorehabilitation stay, while the other studies were performed in the children's natural environment at home or in school. Compared to the children's home environment, inpatient rehabilitation features special adaptations concerning 
optimized infrastructure and "safe" clinical environment on the one hand, and daily routine on the ward serves as an additional training opportunity besides therapy sessions on the other hand. All these aspects might have levelled the differences between the constructs of motor performance, capability, and capacity in inpatient rehabilitation.

Our heterogeneous sample covered a broad range of diagnoses, ages, and mobility levels regarding walking abilities on capacity and performance level - representing the diversity of an inpatient neurorehabilitation setting. We are confident that findings of this study can be transferred to other pediatric neurorehabilitation fields.

\section{CONCLUSION}

The FMS and FAQ are valid measures to evaluate walking ability on the performance level in children undergoing an inpatient neurorehabilitation program. Correlations of the FMS and the FAQ with the comparator measures exceeded the hypothesized lower bounds of .50 and .60 , respectively. However, our hypothesis that the FMS/FAQ would stronger correlate with the other performance measure (the WeeFIM walking item) than with the capacity tests was confirmed for the FMS-500 only.

Measuring performance provides a perspective that is meaningful for the child and the family as the results are directly transferable to the child's behaviour in everyday life. By comparing the performance measures with other frequently used gait measures, we could show that they contribute additional information, which enables health professionals to get a more comprehensive overview of a child's functional walking abilities at little additional cost. This knowledge is essential to confirm whether gait interventions succeeded in achieving performance-based treatment goals. Our study expands the already existing evidence of the measures' validity and demonstrates that they are not only valid to assess functional mobility in the children's usual environment, but also in the inpatient setting. 


\section{ACKNOWLEDGMENTS}

The authors would like to thank all children and parents who participated in this research project. They also thank the physiotherapists who helped collecting the data.

\section{FUNDING}

This work was supported by the Mäxi Foundation, Zurich, Switzerland.

\section{DECLARATION OF INTEREST}

The authors report no potential conflicts of interest.

\section{REFERENCES}

1. World Health Organisation. International Classification of Functioning, Disability and Health: Children \& Youth Version: ICF-CY. Geneva: World Health Organization; 2007.

2. Holsbeeke L, Ketelaar M, Schoemaker MM, Gorter JW. Capacity, capability, and performance: different constructs or three of a kind? Arch Phys Med Rehabil. 2009;90(5):849-855.

3. Palisano RJ, Hanna SE, Rosenbaum PL, Tieman B. Probability of walking, wheeled mobility, and assisted mobility in children and adolescents with cerebral palsy. Dev Med Child Neurol. 2010;52(1):66-71.

4. Ammann-Reiffer C, Bastiaenen CHG, de Bie RA, van Hedel HJA. Measurement Properties of Gait-Related Outcomes in Youth With Neuromuscular Diagnosis: A systematic review. Phys Ther. 2014;94(8):1067-1082.

5. Graham HK, Harvey A, Rodda J, Nattrass GR, Pirpiris M. The Functional Mobility Scale (FMS). J Pediatr Orthop. 2004;24(5):514-520.

6. Harvey A, Graham HK, Morris ME, Baker R, Wolfe R. The Functional Mobility Scale: ability to detect change following single event multilevel surgery. Dev Med Child Neurol. 2007;49(8):603-607.

7. Harvey A, Baker R, Morris ME, Hough J, Hughes M, Graham HK. Does parent report measure performance? A study of the construct validity of the Functional Mobility Scale. Dev Med Child Neurol. 2010;52(2):181-185.

8. Harvey AR, Morris ME, Graham HK, Wolfe R, Baker R. Reliability of the functional mobility scale for children with cerebral palsy. Phys Occup Ther Pediatr. 2010;30(2):139-149. 
9. Novacheck TF, Stout JL, Tervo R. Reliability and Validity of the Gillette Functional Assessment Questionnaire as an Outcome Measure in Children with Walking Disabilities. J Pediatr Orthop. 2000;20(1):75-81.

10. Ammann-Reiffer C, Bastiaenen CHG, de Bie RA, van Hedel HJA. Interrater Reliability of Two Gait Performance Measures in Children with Neuromotor Disorders Across Two Different Settings. Dev Med Child Neurol. 2017;59(11):11581163.

11. Chung M. Encyclopedia of Measurement and Statistics. (Salkind NJ, ed.). London: SAGE Publications, Inc.; 2007.

12. Terwee CB, Mokkink LB, Knol DL, Ostelo RWJG, Bouter LM, de Vet HCW. Rating the methodological quality in systematic reviews of studies on measurement properties: a scoring system for the COSMIN checklist. Qual Life Res. 2012;21(4):651-657.

13. American Thoracic Society. ATS Statement: Guidelines for the Six-Minute Walk Test. Am J Respir Crit Care Med. 2002;166:111-117.

14. Bartels B, de Groot JF, Terwee CB. The Six-Minute Walk Test in Chronic Pediatric Conditions: A Systematic Review of Measurement Properties. Phys Ther. 2013;93(4):529-541.

15. Watson MJ. Refining the Ten-metre Walking Test for Use with Neurologically Impaired People. Physiotherapy. 2002;88(7):386-397.

16. Graser J V, Letsch C, van Hedel HJA. Reliability of timed walking tests and temporo-spatial gait parameters in youths with neurological gait disorders. BMC Neurol. 2016;16(1):15.

17. Russell DJ, Rosenbaum PL, Wright V, Avery LM, eds. Gross Motor Function Measure (GMFM-66 \& GMFM 88) User's Manual. 2nd ed. London: Mac Keith Press; 2013.

18. Harvey A, Robin J, Morris ME, Graham HK, Baker R. A systematic review of measures of activity limitation for children with cerebral palsy. Dev Med Child Neurol. 2008;50(3):190-198.

19. Uniform Data System for Medical Rehabilitation. The WeeFIM II Clinical Guide. Version 6. Buffalo: UDSMR; 2006.

20. Ottenbacher KJ, Taylor ET, Msall ME, et al. The stability and equivalence reliability of the functional independence measure for children (WeeFIM). Dev Med Child Neurol. 1996;38:907-916.

21. Ottenbacher KJ, Msall ME, Lyon NR, et al. Interrater Agreement and Stability of the Functional Independence Measure for Children ( WeeFIMTM ): Use in Children With Developmental Disabilities. Arch Phys Med Rehabil. 1997;78(December):13091315. 
22. Ziviani J, Ottenbacher KJ, Shephard K, Foreman S, Astbury W, Ireland P. Concurrent Validity of the Functional Independence Measure for Children (WeeFIM) and the Pediatric Evaluation of Disabilities Inventory in Children with Developmental Disabilities and Acquired Brain Injuries. Phys Occup Ther Pediatr. 2001;21(2):91101.

23. Beaton DE, Bombardier C, Guillemin F, Ferraz MB. Guidelines for the Process of Cross-Cultural Adaptation of Self-Report Measures. Spine. 2000;25(24):31863191.

24. Peter WF, Loos M, van den Hoek J, Terwee CB. Validation of the Animated Activity Questionnaire (AAQ) for patients with hip and knee osteoarthritis: comparison to home-recorded videos. Rheumatol Int. 2015;35(8):1399-1408.

25. Ottenbacher KJ, Msall ME, Lyon N, et al. The WeeFIM Instrument: Its Utility in Detecting Change in Children With Developmental Disabilities. 2000.

26. Sanders JO, McConnell SL, King R, et al. A prospective evaluation of the WeeFIM in patients with cerebral palsy undergoing orthopaedic surgery. $J$ Pediatr Orthop. 2006;26(4):542-546.

27. de Vet HCW, Terwee CB, Mokkink LB, Knol DL. Measurement in Medicine. Cambridge: Cambridge University Press; 2011.

28. Tieman BL, Palisano RJ, Gracely EJ, Rosenbaum PL, Chiarello LA, Neil MEO. Changes in Mobility of Children with Cerebral Palsy Over Time and Across Environmental Settings. 2004:109-128. 


\section{CHAPTER 4}

\section{INTERRATER RELIABILITY OF TWO GAIT PERFORMANCE MEASURES IN CHILDREN WITH NEUROMOTOR DISORDERS ACROSS TWO DIFFERENT SETTINGS}

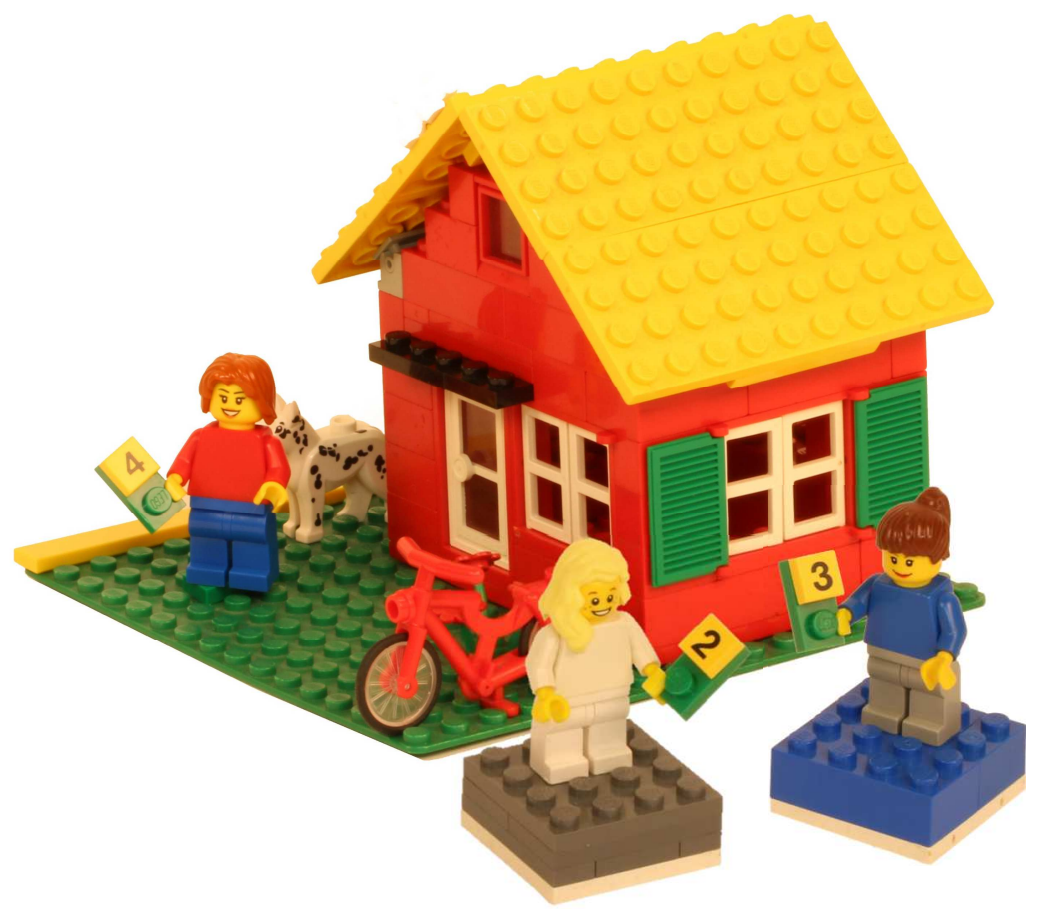

This chapter has been published as:

Ammann-Reiffer C, Bastiaenen CHG, de Bie RA, van Hedel HJA. Interrater reliability of two gait performance measures in children with neuromotor disorders across two different settings. Dev Med Child Neurol. 2017;59(11):1158-1163. 


\section{ABSTRACT}

AIMS: To examine the interrater agreement of the two gait performance measures - "Functional Mobility Scale" (FMS) and "Gillette Functional Assessment Questionnaire - walking scale" (FAQ) - within health professionals and parents in children with neuromotor disorders, measured in an inpatient setting and at home.

METHOD: Seventy-one children with a neuromotor diagnosis (27 females; median age 12 years 11 months (interquartile range 4 years-10 months) were consecutively recruited when starting an inpatient active gait rehabilitation programme. Physiotherapists and nurses independently scored the level of children's gait performance with the FMS and the FAQ, while parents' scores regarding the children's gait performance at home were obtained by interview or telephone call at the same measurement points.

RESULTS: Linear weighted kappa coefficients were substantial to almost perfect for all comparisons. Kappa coefficients ranged from 0.62 to 0.85 for the FMS-5, from 0.79 to 0.92 for the FMS-50, from 0.83 to 0.90 for the FMS-500, and from 0.69 to 0.77 for the FAQ. Friedman tests did not reveal significant differences between the different rater groups.

INTERPRETATION: The unexpectedly high level of interrater agreement between parents, physiotherapists, and nurses demonstrates that the FMS and FAQ can reliably assess gait performance in an inpatient setting. Inpatient scores correspond well to the children's performance in their usual environment. 


\section{INTRODUCTION}

In inpatient settings, tests for the evaluation of a child's walking abilities are predominantly carried out in a standardized environment. Thus, the results of these tests primarily reflect a child's gait function on either the capability or the capacity level. Gait performance, which characterizes an individual's actual level of functional mobility in his or her current environment, is far less considered in studies evaluating the effect of different gait training programmes. ${ }^{1}$ The International Classification of Functioning, Disability and Health for Children and Youth provides a theoretical framework for describing and measuring health and health-related states. ${ }^{2}$ It classifies outcome into the four domains: "body structure and function", "activity", "participation", and "environmental and personal factors". Instruments with sound psychometric properties reflecting the activity and participation domain are increasingly available for use in children, but outcomes of gait training programmes or lower limb orthopaedic surgery in ambulatory children with cerebral palsy (CP) are predominantly evaluated on the body structure and function level. ${ }^{1,3}$

Although there appears to be a clear relationship between motor performance and motor capacity, motor performance is only partly reflected by the motor capacity level as children with a similar gross motor capacity level can differ in terms of gait performance across different settings. ${ }^{4}$ Many environmental factors may influence motor performance either in a negative or positive way. Motivation, assistive devices, availability of transportation, distances to be covered, physical accessibility, obstacles, time constraints, family support or peer acceptance are just a few examples of these aspects.

Environmental settings play an important role when considering mobility in everyday life. While there is a relatively constant environment at home, in which it is possible to arrange various factors to enhance mobility, children have to deal with far more inconsistent or unpredictable elements at school or in the community. Accordingly, different environmental settings affect mobility methods, as well as dependence on adult assistance, in children with CP. ${ }^{5}$

Although achieving a child's independence in daily life activities may be a major goal of rehabilitation, there is a paucity of reliable, valid, and responsive outcome 
measures that evaluate gait performance in children with neuromotor disorders in daily life and across different settings. ${ }^{6}$ As direct observation of a child's usual mobility methods is often not feasible, the few existing tools usually rely on parent reports, which are a realistic and valid way to gather data reflecting gait performance of a child. ${ }^{7}$ The Functional Mobility Scale (FMS) and the Gillette Functional Assessment Questionnaire - walking scale (FAQ) are examples of such tools. ${ }^{8,9}$ The FMS describes the level of a child's functional mobility by scoring the assistive device that is used in everyday life. ${ }^{8}$ It is usually administered by a clinician in a semistructured interview using child or parent report and is scored over 5, 50, and 500 meters - representing typical distances covered by children at home, in school, and the wider community. For each distance, the type of assistance required is rated on an ordinal scale from 1 (uses a wheelchair) to 6 (independent walking without an assistive device on all surfaces). ${ }^{8} \mathrm{~A}$ comparison between the FMS score obtained by parent interview of 4 to 18 -year-old children with $\mathrm{CP}$ and direct observation of the methods of mobility used around and outside their home and at school resulted in substantial agreement (weighted kappa $0.71-0.76) .{ }^{10}$ Regarding interrater reliability, the FMS demonstrated very high agreement (intraclass correlation coefficient (ICC) $=0.94-0.98$ ) when scored by three orthopaedic surgeons in 4 to 18-year-old children with CP attending an orthopaedic outpatient clinic. ${ }^{8}$ The comparison of parent or child interview-based scores of physiotherapists and orthopaedic surgeons in 2.5 to 18-year-old children with $\mathrm{CP}$ attending a CP outpatient clinic resulted in kappa values of 0.86 to 0.92 - with similar levels of agreement when administering the scale in person or by telephone call. ${ }^{10}$ The scale showed longitudinal changes after multilevel orthopaedic surgery and correlated well with changes in other measures of functional activity, measured with both questionnaires (Spearman's rank correlation coefficient $\left.r_{s}=0.75-0.83\right)$ and activity monitors $\left(r_{s}=0.78-0.89\right)$, providing evidence of its responsiveness. ${ }^{8}$

The FAQ involves a range of walking abilities, describing various levels of mobility on a 10-level ordinal scale, with the levels differing in environment, terrains, or obstacles. ${ }^{9}$ It is given to the parent or the child to read and identify the child's usual level of function. Its reliability has been evaluated in 2 to 17 -year-old children with 
various neuromotor diagnoses. Intrarater reliability of parent interview-derived scores with an average time span of 14 weeks in between was high (ICC=0.92). ${ }^{9}$ Interrater reliability, where parent- and teacher-derived scores were compared, reached ICCs of 0.81 to $0.92 .{ }^{9}$ The average time between ratings varied between 5 and 8 weeks. The FAQ's content and concurrent validity have been assessed by comparing the scale with other measures of gait function. ${ }^{9}$ Only children from level 6 to 10 of the FAQ were represented in the study sample. ${ }^{9}$

So far, both the FMS and the FAQ have only been used in outpatient settings. ${ }^{8,9}$

There are some potential difficulties to be considered in case these tools are to be applied in the inpatient setting: daily routines in a rehabilitation environment are generally more structured than in everyday life. Further, rehabilitation health professionals assess a child's gait performance during inpatient rehabilitation, whereas the parents rate it at home. The question is whether the level of interobserver agreement between the different rater groups is of an a priori defined acceptable level, despite their marked differences in background and environment. In daily practice, the measures could then be applied either at home by the parents or in the clinic by health professionals.

We are not aware of any study that investigated children's gait performance in an inpatient rehabilitation setting or compared the reliability between different settings. Hence, the aim of our study was to examine the interrater reliability of the FMS and the $F A Q$ in children and adolescents with neuromotor disorders between different health professionals (physiotherapists and nurses) in an inpatient neurorehabilitation setting and between the health professionals and the parents at home.

\section{METHOD}

\section{Study design and setting}

This study was designed as a cross-sectional study nested within an overall longitudinal study that evaluated different psychometric properties as validity and responsiveness of the same two measurement tools. The measurements were performed at the Rehabilitation Center Affoltern am Albis of the University Children's Hospital Zurich and at the children's home in Switzerland. Our center provides in- 
and outpatient treatment for children and adolescents with acquired and congenital diseases or injuries, with a predominant proportion of neurological diagnoses. While the reasons for an admission to the rehabilitation center can vary, the most common are postoperative treatment, improving independence in everyday life activities, and optimizing orthopaedic devices and/or dosage of medication to the individual needs of the patient. The center operates three inpatient wards, which are partly organized as living groups, where not only activities of everyday life, but also social interactions play an important role. The daily schedule of the children is adjusted to the children's individual needs and can include physiotherapy, speech and language therapy, occupational therapy, sport groups, and school or kindergarten lessons. The study was approved by the Ethics Committee Zurich and registered on ClinicalTrials.gov (NCT02237222).

\section{Participants}

Children and adolescents were eligible for the study if they (1) were between 6 and 18 years of age; (2) had a neuromotor diagnosis; (3) were inpatients at the rehabilitation center; and (4) their rehabilitation programme contained therapy sessions in which they trained their gait function actively. Children meeting these inclusion criteria were consecutively recruited for the study between April 2014 and January 2016, provided informed consent was obtained from the legal guardians and the child. Children of non-German-speaking parents were excluded from the study.

\section{Study procedure}

As the study was carried out in the German-speaking part of Switzerland, the German versions of the two questionnaires were used (see Appendix). In short, for each questionnaire, the original English version was translated into German, adhering to the guidelines of the American Association of Orthopaedic Surgeons Outcomes Committee. ${ }^{11}$ Two native German-speaking health professionals translated the questionnaire independently from each other. These versions were synthesized into one consensus version, which was then back-translated into English by a professional translation agency and a native English-speaking person, both blinded to the original version and naïve to the construct to be measured. 
Finally, we obtained approval of the back translation by the authors of the original questionnaires.

Reliability was assessed at two different time points: once at the start of active gait rehabilitation ( $\left.T_{0}\right)$ and once at the end of the rehabilitation stay $\left(T_{1}\right)$. This procedure was chosen to evaluate whether the time the health professionals had worked with the child would have an influence on their ratings. To characterized the moment when children began to train their ambulatory abilities in physiotherapy sessions, were allowed to at least partially load both legs after having undergone orthopaedic surgery, and, if needed, when walking aids for daily life had been provided. $\mathrm{T}_{1}$ signified the end of the rehabilitation stay or the moment when physiotherapy sessions were ceased because all physiotherapeutic goals had been reached. Accordingly, the time interval between $T_{0}$ and $T_{1}$ could vary depending on the children's length of rehabilitation stay and the content of the therapy programme.

The reliability measurements for each point in time $\left(T_{0}\right.$ and $\left.T_{1}\right)$ were carried out within a maximal time frame of 7 days to ensure that the children's condition was stable across these measurements. Only the parents' ratings at $\mathrm{T}_{1}$ (after rehabilitation) were set at 1 to 2 weeks after discharge, to allow the parents some time to assess their children's current level of gait performance at home. We assumed that the children's health status and gait performance had remained stable in the first weeks after discharge unless the parents stated otherwise.

At the rehabilitation center, children were assessed independently by two different groups of raters. The physiotherapists and the nurses mainly responsible for the daily care of the children independently completed the German FMS and FAQ at $T_{0}$ and $\mathrm{T}_{1}\left(=\mathrm{Pt}_{0} / \mathrm{Pt}_{1}\right.$ and $\mathrm{Nu} / \mathrm{Nu}_{1}$, respectively). As none of the raters had previous experience with the FMS or FAQ, they received brief protocolled instructions beforehand. To ensure that therapists/nurses filled in the forms accurately, they were assisted by an independent research fellow. The research fellow performed a standardized interview to ensure that the raters adhered to the standardized instructions of the authors of the original English versions. The parent ratings were either obtained in the form of a personal interview or by a telephone call, also strictly following a protocol regarding instructions and questions on the questionnaires 
$\left(=\mathrm{Pa}_{0} / \mathrm{Pa}_{1}\right)$. To reduce the risk of bias, the members of the different rater groups did not have any mutual contact during the measurement process. If parents mentioned an observed change in their child's motor function between discharge and the moment of their rating, parent scores were not included in the analysis. Whenever possible, parents were interviewed by the same research fellow at $\mathrm{Pa} 0$ and $\mathrm{Pa}_{1}$. $\mathrm{A}$ $\mathrm{Pa}$ o rating could only be completed for children who had directly been referred from home to the rehabilitation center (Fig. 4.1.)

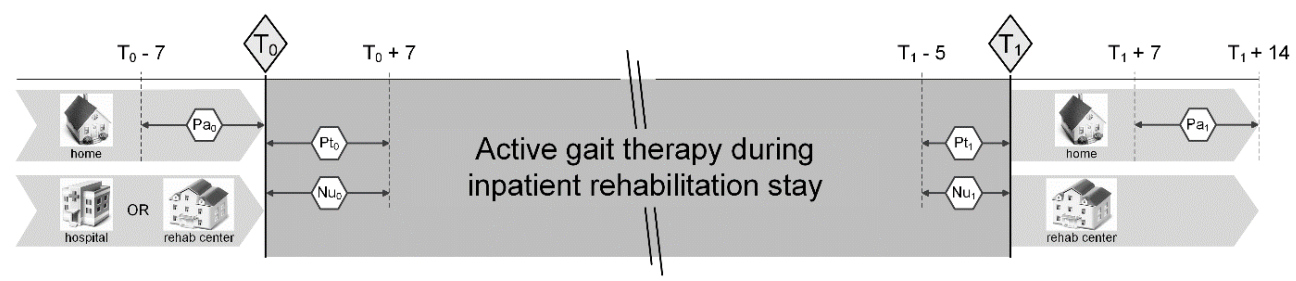

Figure 4.1. Study design and measurement moments.

Reliability measurements of the Functional Mobility Scale and Gillette Functional Assessment Questionnaire - walking scale at two different measurement time points with three different rater groups. $T_{0}$, measurement at start of active gait rehabilitation; $T_{1}$, measurement at end of rehabilitation; $P a$, parent ratings; Pt, physiotherapist ratings; $\mathrm{Nu}$, nurse ratings.

\section{Statistical analysis}

All data were captured in Microsoft Office Access 2007 (Microsoft, Redmond, WA, USA) and statistical analyses were conducted using SPSS version 24.0 (IBM SPSS Statistics, Armonk, NY, USA). Descriptive statistics are presented for relevant characteristics of the study population and the outcome measures. Linear weighted kappa statistics with $95 \%$ confidence intervals were calculated between the scores of the physiotherapists and the nurses in order to analyse the interrater agreement in the inpatient environment. To evaluate the interrater agreement across different settings, the same analyses were made by comparing the parents' scores with those of the physiotherapists and the nurses. As we assumed two sources of variation in our data (different environmental settings and different groups of raters), we set the acceptance level for interrater agreement at 0.6 , which is in agreement with the definitions of Landis and Koch, ${ }^{12}$ who considered 0.61 to 0.8 as substantial and 0.81 to 1 as almost perfect. Potential differences between rater groups were examined with Friedman tests. We made separate analyses for $T_{0}$ (before start of treatment) 
and $\mathrm{T}_{1}$ (after treatment). Missing data were not replaced. We set the statistical level of significance at 0.05 .

\section{RESULTS}

Characteristics of the 71 participants are listed in Table 4.1. Mean (SD) rehabilitation length was 10 (9) weeks (range 2-45 weeks), and the mean (SD) time between $T_{0}$ and $\mathrm{T}_{1}$ was 7 (8) weeks (2-44 weeks). Measurements of all raters were collected within a mean (SD) of 2.5 (3.2) days for $T_{0}$ and 8.1 (4.4) days for $T_{1}$. As many children were directly referred from a hospital to our rehabilitation center, To parent scores were collected in 35 children only. Other reasons for missing values were cessation of physiotherapy sessions before discharge $(n=5)$, alteration in observed motor function between the moment of discharge and $\mathrm{Pa}_{1}(n=3)$, and unavailability of the rater at the assessment time point $(n=3)$. Additionally, measurements only took place at a single time point in five children. See Figure 4.2. for exact numbers and score distributions.

Table 4.1. Characteristics of the study sample

\begin{tabular}{ll}
\hline Age & \\
Median (IOR) & $12 \mathrm{y} 11 \mathrm{mo}(4 \mathrm{y} 10 \mathrm{mo})$ \\
Range & $6 \mathrm{y}-18 \mathrm{y} 6 \mathrm{mo}$ \\
Sex $(n)$ & 27 \\
Female & 44 \\
Male & \\
Diagnoses ( $n)$ & 38 \\
Cerebral palsy & 7 \\
GMFCS level I & 12 \\
GMFCS level II & 13 \\
GMFCS level III & 6 \\
GMFCS level IV & 9 \\
TBI $(n)$ & 7 \\
Genetic disorders $(n)$ & 6 \\
Stroke $(n)$ & 3 \\
Encephalitis $(n)$ & 8 \\
Other $(n)$ & 8 \\
\hline
\end{tabular}

IQR, interquartile range; GMFCS, Gross Motor Function Classification System; TBI, traumatic brain injury. 


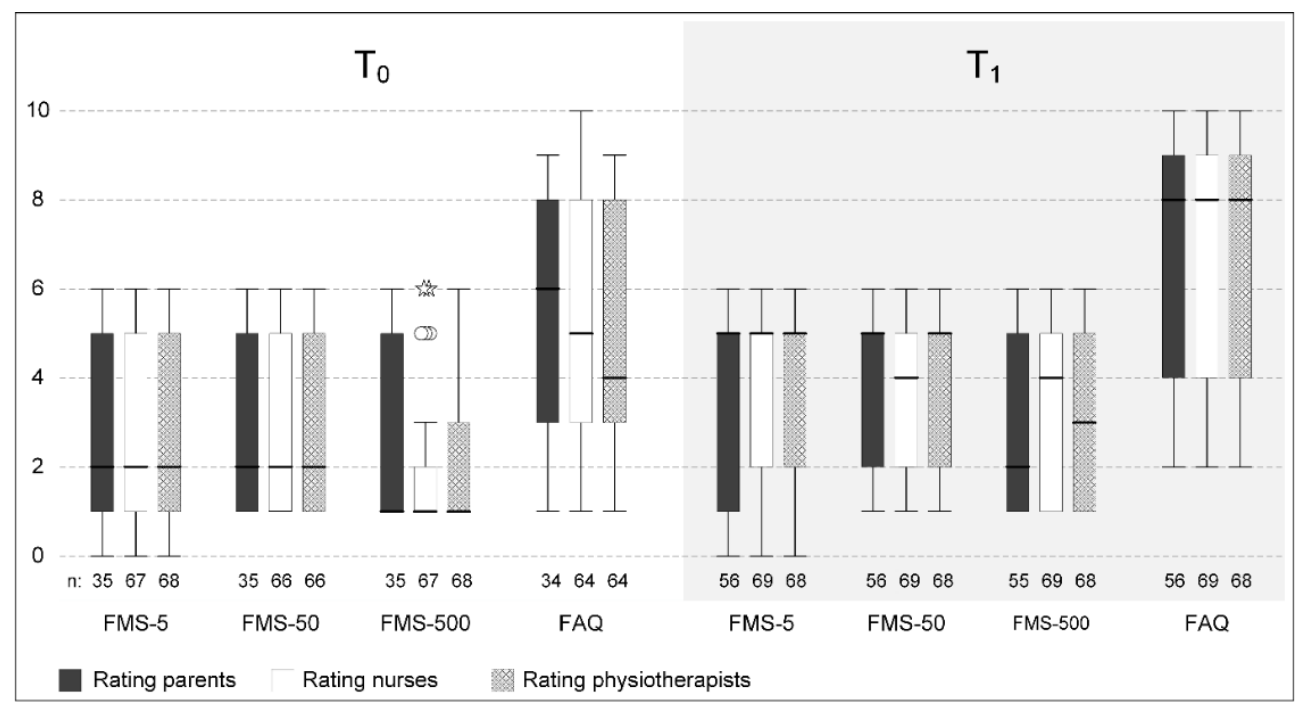

Figure 4.2. Score distribution of motor performance measures. Rating of the Functional Mobility Scale (FMS) and the Gillette Functional Assessment Questionnaire - walking scale (FAQ) at two different measurement time points by the three different rater groups.

T0, measurement at start of active gait rehabilitation; T1, measurement at end of rehabilitation; FMS$5 / 50 / 500$, FMS over 5/50/500 meters $(0=$ crawling).

Kappa coefficients regarding interrater reliability were substantial to almost perfect, ${ }^{12}$ and were all above our stated acceptance level of 0.6 (Table 4.2.). The lowest values were attained for the FMS-5 at $T_{0}$ and the FAQ at $T_{0}$ and $T_{1}$. Based on the $95 \%$ confidence intervals, the kappa coefficients did not differ significantly at the two assessment points. Friedman tests did not reveal any significant differences between the three rater groups $(0.06<p$-value $<0.96)$.

Table 4.2. Linear weighted kappa and $95 \%$ confidence intervals $(\mathrm{Cl})$ of rater group combinations at two different measurement time points.

\begin{tabular}{|c|c|c|c|c|c|c|}
\hline & \multicolumn{2}{|c|}{ Parent-Nurse } & \multicolumn{2}{|c|}{ Parent-Physiotherapist } & \multicolumn{2}{|c|}{ Physiotherapist-Nurse } \\
\hline & $\begin{array}{l}\text { Linear weighted } \\
\text { kappa }\end{array}$ & $95 \% \mathrm{Cl}$ & $\begin{array}{l}\text { Linear weighted } \\
\text { kappa }\end{array}$ & $95 \% \mathrm{Cl}$ & $\begin{array}{l}\text { Linear weighted } \\
\text { kappa }\end{array}$ & $95 \% \mathrm{Cl}$ \\
\hline \multicolumn{7}{|l|}{$\mathrm{T}_{0}$} \\
\hline FMS-5 & 0.74 & 0.590 .88 & 0.62 & 0.440 .79 & 0.69 & $0.57-0.81$ \\
\hline FMS-50 & 0.82 & $0.71-0.93$ & 0.79 & $0.66-0.92$ & 0.83 & $0.75-0.92$ \\
\hline FMS-500 & 0.87 & 0.770 .97 & 0.87 & $0.78 \quad 0.96$ & 0.90 & 0.830 .98 \\
\hline FAO & 0.77 & $0.67-0.87$ & 0.69 & $0.57-0.81$ & 0.75 & $0.68-0.82$ \\
\hline \multicolumn{7}{|l|}{$\mathrm{T}_{1}$} \\
\hline FMS-5 & 0.82 & $0.72-0.92$ & 0.83 & $0.74-0.92$ & 0.85 & $0.77-0.93$ \\
\hline FMS-50 & 0.84 & 0.750 .93 & 0.90 & 0.840 .97 & 0.92 & $0.85-0.98$ \\
\hline FMS-500 & 0.85 & $0.75-0.95$ & 0.83 & $0.73-0.94$ & 0.84 & $0.75-0.94$ \\
\hline FAQ & 0.74 & 0.650 .84 & 0.77 & 0.690 .85 & 0.74 & $\begin{array}{llll}0.67 & 0.81\end{array}$ \\
\hline
\end{tabular}

FMS-5/50/500, Functional Mobility Scale over 5/50/500m; FAQ, Gillette Functional Assessment Questionnaire - walking scale; To, measurement at start of active gait rehabilitation; $T_{1}$, measurement at end of rehabilitation. 


\section{DISCUSSION}

We determined the interrater reliability of the FMS and the FAQ of physiotherapists, nurses, and parents of children and adolescents with neuromotor disorders who were undergoing an inpatient gait rehabilitation programme. Our study encompassed children over the whole spectrum of the FMS and FAQ scales. Interrater agreement exceeded our predefined acceptance level of 0.6 and was substantial to almost perfect for all comparisons. ${ }^{12}$

Our results showed a slightly lower interrater agreement than those reported in previous studies..$^{8-10}$ This is not surprising, as our study not only evaluated the interrater agreement of a very heterogeneous group of raters - i.e. different health professionals and parents - but also dealt with a heterogeneous group of children, as we aimed for a reflection of daily practice, in which more sources of variation in the data are inevitable. While previous studies showed that different environmental settings affect children's mobility and that gait performance can differ across different settings, $4,5,13$ the high levels of agreement between the parents and the health professionals provide evidence that gait performance as assessed with the FMS and FAQ around admission or discharge corresponds well between the inpatient setting and the children's usual environment. As scores are influenced by the people who perform the assessments, as well as the environment in which the assessment is performed, the substantial agreement is positively surprising and indicates that the scores can be measured and interpreted in the examined settings. ${ }^{14}$

Although kappa coefficients did not differ significantly, there was a tendency to a higher agreement at $T_{1}$ for the FMS-5 and FMS-50 ratings, especially when physiotherapists were involved in the rater combination. One reason might be that the health professionals had a much better impression of the children's gait performance at the end of rehabilitation than at the beginning, because they have had more possibilities to observe the children in the course of the rehabilitation stay. This made it easier for them to rate the gait performance at $T_{1}$.

Interestingly, interrater agreement for the FMS-500 at To was very high. Although in practice the health professionals had only rarely the occasion to observe the children 
outdoors, many children relied on the wheelchair for outdoor mobility over longer distances, which made the rating in those children unambiguous.

For the $F A Q$, interrater agreement did not differ largely at $T_{0}$ and $T_{1}$, except for the agreement between parents and physiotherapists, which increased markedly at $\mathrm{T}_{1}$. Again, this might be owing to the increased observation possibilities. Discrepancies between rater groups were mainly observable at the lower end of the scale, when parents stated that their child would need help to do some steps while health professionals rated them higher. Apparently, these children seemed to walk with the help of their parents at home, while they managed to do some steps without help in the rehabilitation setting. Further, some difficulties arose at the higher end of the scale, where raters had to judge on which terrain the child was able to walk independently and where assistance was required. These parameters seemed to cause uncertainty in all rater groups. Part of the observed differences in the FAQ might have resulted from the use or non-use of walking aids within the different environments, because the FAQ does not take into account the walking aid that is used.

With the exception of the FMS-5 at $T_{0}$, nearly all of the FMS values showed almost perfect interrater agreement, whereas the $F A Q$ attained only substantial agreement across all time points and settings. This might be an important decision criteria for the clinician, when time restraints might not justify using both tools.

Different studies underline the relevance of including performance tools into the routine assessment of patients: Bjornson et al. suggested that interventions in ambulatory children with $\mathrm{CP}$ should focus more on improving their performance, as the relation between children's capacity and participation in life was significantly mediated by their performance..$^{15}$ When families were asked what kind of changes they desired after an intervention they identified areas of importance which are also covered in the performance domain of the International Classification of Functioning, Disability and Health: Children and Youth Version. ${ }^{16}$

We address some methodological considerations: FMS and FAQ have originally been developed for the outpatient setting to interview parents on their child's functional mobility. 8,9 We used the original test administration methods (i.e. interview 
or telephone call) to obtain parents' ratings, as a previous study showed that reliability of FMS scores was not influenced by these different methods. ${ }^{10}$ The study also showed that interrater agreement was high, irrespective of whether the FMS was administered on the same or different days, which was the case in our study. ${ }^{10}$ Nurses and physiotherapists scored the questionnaires based on their observations instead of interviewing the parents to enable us to assess the children's actual gait performance during their inpatient stay. Although we are not aware of other studies in which the FMS or FAQ were administered this way, we chose this approach to assess the psychometric properties of the two performance tools under realistic and feasible conditions for an inpatient setting.

To evaluate the effectiveness of inpatient rehabilitation programs on gait performance, information on the tools' responsiveness and minimal clinically important difference in this particular setting is also vital and should be subject to further studies.

\section{CONCLUSION}

Despite the large variation that we have been faced with by assessing gait performance in a realistic inpatient and home setting, the FMS and FAQ showed high levels of interrater agreement in children with neuromotor disorders undergoing an inpatient rehabilitation programme. Interrater agreement between parents, physiotherapists, and nurses was substantial to almost perfect and above the threshold of 0.6 at both the start and the end of active gait rehabilitation. The results demonstrate that gait performance can be reliably assessed by parent report in the home setting and by health professionals in an inpatient setting. Interestingly, these gait performance levels are transferable between the home and the inpatient settings, which is clinically relevant as it allows us to estimate improvements in gait performance during daily life in patients undergoing rehabilitation of gait. With that, the ratings on the FMS and FAQ at discharge can, for example, be used to instruct parents about the level of function they should expect from their child in everyday life. 


\section{ACKNOWLEDGMENTS}

The authors gratefully acknowledge all the parents, nurses, and physiotherapists who participated in the data collection, as well as Corina Klöti, who helped collecting the data. We also gratefully acknowledge funding by the Mäxi Foundation, Zurich, Switzerland.

\section{DECLARATION OF INTEREST}

The authors have stated that they had no interests which might be perceived as posing a conflict or bias.

\section{REFERENCES}

1. Zwicker JG, Mayson TA. Effectiveness of Treadmill Training in Children With Motor Impairments : An Overview. Pediatr Phys Ther. 2010;22:361-377.

2. World Health Organisation. International Classification of Functioning, Disability and Health: Children \& Youth Version: ICF-CY. Geneva: World Health Organization; 2007.

3. Wilson NC, Chong J, Mackey AH, Stott NS. Reported outcomes of lower limb orthopaedic surgery in children and adolescents with cerebral palsy: a mapping review. Dev Med Child Neurol. 2014;56(9):808-814.

4. Tieman BL, Palisano RJ, Gracely EJ, Rosenbaum PL. Gross Motor Capability and Performance of Mobility in Children With Cerebral Palsy: A Comparison Across Home, School, and Outdoors/Community Settings. Phys Ther. 2004;84:419-429.

5. Palisano RJ, Tieman BL, Walter SD, et al. Effect of environmental setting on mobility methods of children with cerebral palsy. Dev Med Child Neurol. 2003;45(2):113-120.

6. Ammann-Reiffer C, Bastiaenen CHG, de Bie RA, van Hedel HJA. Measurement Properties of Gait-Related Outcomes in Youth With Neuromuscular Diagnosis: A systematic review. Phys Ther. 2014;94(8):1067-1082.

7. Harvey A, Baker R, Morris ME, Hough J, Hughes M, Graham HK. Does parent report measure performance? A study of the construct validity of the Functional Mobility Scale. Dev Med Child Neurol. 2010;52(2):181-185.

8. Graham HK, Harvey A, Rodda J, Nattrass GR, Pirpiris M. The Functional Mobility Scale (FMS). J Pediatr Orthop. 2004;24(5):514-520.

9. Novacheck TF, Stout JL, Tervo R. Reliability and Validity of the Gillette Functional Assessment Questionnaire as an Outcome Measure in Children with Walking Disabilities. J Pediatr Orthop. 2000;20(1):75-81. 
10. Harvey AR, Morris ME, Graham HK, Wolfe R, Baker R. Reliability of the functional mobility scale for children with cerebral palsy. Phys Occup Ther Pediatr. 2010;30(2):139-149.

11. Beaton DE, Bombardier C, Guillemin F, Ferraz MB. Guidelines for the Process of Cross-Cultural Adaptation of Self-Report Measures. Spine (Phila Pa 1976). 2000;25(24):3186-3191.

12. Landis JR, Koch GG. The Measurement of Observer Agreement for Categorical Data. Biometrics. 1977;33(1):159-174.

13. Palisano RJ, Hanna SE, Rosenbaum PL, Tieman B. Probability of walking, wheeled mobility, and assisted mobility in children and adolescents with cerebral palsy. Dev Med Child Neurol. 2010;52(1):66-71.

14. Streiner DL, Norman GR. "Precision" and "accuracy": Two terms that are neither. J Clin Epidemiol. 2006;59(4):327-330.

15. Bjornson KF, Zhou C, Stevenson R, Christakis DA. Capacity to participation in cerebral palsy: Evidence of an indirect path via performance. Arch Phys Med Rehabil. 2013;94(12):2365-2372.

16. Vargus-Adams JN, Martin LK. Domains of importance for parents, medical professionals and youth with cerebral palsy considering treatment outcomes. Child Care Health Dev. 2011;37(2):276-281. 

CHAPTER 5

\section{RESPONSIVENESS AND MINIMAL IMPORTANT}

CHANGE OF TWO GAIT PERFORMANCE MEASURES IN CHILDREN WITH NEUROMOTOR DISORDERS

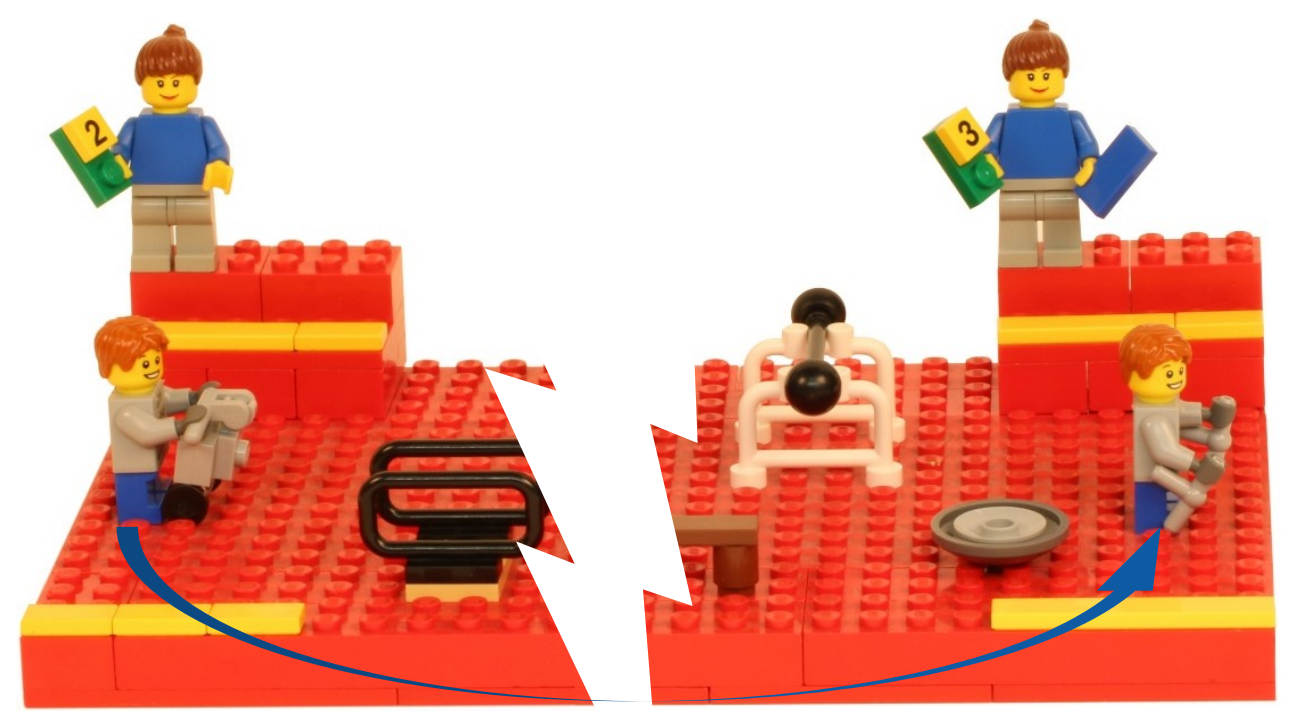

This chapter has been accepted as:

Ammann-Reiffer C, Bastiaenen CHG, van Hedel HJA. Responsiveness and minimal important change of two gait performance measures in children with neuromotor disorders. Dev Med Child Neurol. 


\section{ABSTRACT}

AIMS: To examine responsiveness and minimal important change (MIC) of the two gait performance measures "Functional Mobility Scale" (FMS) and "Gillette Functional Assessment Questionnaire - walking scale" (FAQ) in a pediatric inpatient setting.

METHOD: Sixty-four children and adolescents with a neuromotor disorder, e.g. cerebral palsy, traumatic brain injury or stroke, (25 females; mean (SD) age 12.5 (3.2) years) were recruited. Physiotherapists scored the FMS and FAQ at start and end of active gait rehabilitation. Change scores were compared to changes in gait capacity tests, the walking item of the Functional Independence Measure for Children (WeeFIM), and a global rating scale (GRS) on the physiotherapists' perceived change regarding the child's functional mobility. The GRS was also used to define the MIC. Hypotheses were formulated a priori.

RESULTS: Change scores of the FMS/FAQ correlated between 0.35-49 with those of the capacity tests, $0.54-0.76$ with the WeeFIM walking item change scores, and 0.57-0.76 with the GRS. The MIC for FMS and FAQ was 0.5 and 1.5 levels, respectively.

INTERPRETATION: FMS and FAQ can illustrate change in inpatient gait performance of children and adolescents with neuromotor disorders. An improvement of 1 level in the FMS and 2 levels in the FAQ is considered as a clinically meaningful change. 


\section{INTRODUCTION}

Clinicians and researchers rely on various outcome instruments when they evaluate the effects of interventions that aim at improving walking abilities in children with neuromotor disorders. With regard to the International Classification of Functioning, Disability and Health for Children and Youth (ICF-CY), most of these instruments can be linked to the body function domain or the capacity level of the activity domain. ${ }^{1,2}$ Capacity describes what a child can do in a standardized controlled environment. ${ }^{3}$ Although walking capacity has an impact on walking performance (i.e. how a child actually moves around in his/her current environment in daily life), environmental and personal factors seem to play an even more important role and are valuable to integrate in constructs measuring walking ability. ${ }^{4}$ Within this perspective, walking performance is defined as how children actually move around in their current environment in everyday life. ${ }^{3}$

The Functional Mobility Scale (FMS) and the Gillette Functional Assessment Questionnaire - walking scale (FAQ) are examples of the few tools that assess walking ability on a performance level. The FMS describes the level of a child's functional mobility in everyday life over 5,50 , and 500 meters, representing the home, school, and community settings, respectively. ${ }^{5}$ For each distance, an ordinal rating from 1 to 6 is assigned depending on the amount of assistance required for the child's mobility. It is usually administered by a clinician in a semi-structured interview using child or parent report. ${ }^{5}$

The FAQ assesses the level of a child's functional mobility on an ordinal scale by describing various levels of mobility differing in environment, terrains, or obstacles. ${ }^{6}$ The scale ranges from 1 (the child cannot take any steps at all) to 10 (the child walks, runs, and climbs on level and uneven terrain without difficulty). ${ }^{6}$ It is a parent-/selfreport measure to identify the child's usual level of function. Both, the FMS and the $\mathrm{FAQ}$ are easy to use and require no specific assessor training. ${ }^{7}$

To accurately measure and interpret a child's performance in clinical practice and research, assessments should be sufficiently valid, reliable, and responsive. The COSMIN (COnsensus-based Standards for the selection of health Measurement INstruments) group defined responsiveness as 'the ability of an instrument to detect 
change over time in the construct to be measured'. ${ }^{8}$ Another relevant aspect is the degree to which one can assign qualitative meaning to scores or change scores, or, in other words, the interpretability of instruments. ${ }^{9}$ Minimal important change (MIC) is one aspect of interpretability and is defined as 'the smallest change in score in the construct to be measured which patients perceive as important'. ${ }^{8}$ For this, change scores are related to a clinically meaningful reference (i.e. an anchor-based approach) from a patient's or a clinician's perspective. Important is that the anchor reflects the construct that the target instrument aims to measure. ${ }^{9}$

The FMS correlated moderately to very strongly ( $r s=0.58-0.89)$ with other gait measures in in- and outpatient settings..$^{5,10}$ Interrater agreement was substantial to almost perfect in ambulatory children with neuromotor disorders. ${ }^{5,11,12}$ Furthermore, FMS scores obtained by parent interview and direct observation agreed substantially, ${ }^{13}$ and it appears to be responsive in an outpatient setting, $, 5,6,11-14$

The FAQ showed almost perfect intra- and interrater reliability as well as content and concurrent validity when compared to other measures of gait function in children with various neuromotor disorders. ${ }^{6}$ It has been utilized as an outcome measure after various interventions. ${ }^{15,16}$ However, we are unaware of studies focusing on the evaluation of the FAQ's responsiveness in a psychometric study design in out- or inpatients.

Thus, our aim was to investigate responsiveness of the FMS and FAQ in children and adolescents with neuromotor disorders in an inpatient neuro-rehabilitation setting by examining the correlations between changes over time in FMS/FAQ scores to changes over time in standard functional and capacity measures. We had formulated the following four hypotheses a-priori:

(1) Correlations between the change scores of the FMS/FAQ and the change scores of the comparator tests are positive and moderate to strong, with Spearman correlation coefficients $(r s) \geq 0.4$.

(2) Correlations between a global rating scale (GRS) quantifying the therapists' perceived change of the children's functional mobility in everyday life and the change scores of the FMS/FAQ are stronger than the correlations between the GRS and the change scores of the capacity measures. 
(3) The values of the area under the receiver operating characteristic (ROC) curves (AUC) between the dichotomized GRS (no change versus change (improvement)) and the FMS/FAQ are $\geq 0.7$. This threshold would indicate that the measures could discriminate between children whose walking performance remained unchanged versus those who changed (improved).

(4) The change scores of the FMS/FAQ in children who had received surgery are larger than in non-operated children.

Further, we determined the MIC of FMS/FAQ by relating the change scores of the two measures to the treating physiotherapists' perceived change of the children's functional mobility.

\section{METHOD}

\section{Study design and setting}

This prospective longitudinal psychometric study used a construct and criterion approach to evaluate responsiveness and an anchor-based approach to assess the MIC of two gait performance measures. Measurements were performed at the Rehabilitation Center Affoltern am Albis of the Children's University Hospital Zurich, Switzerland. The center provides in- and outpatient treatment for children and adolescents with predominantly congenital and acquired neurological disorders. Most frequent reasons for an admission to the rehabilitation center are postsurgical treatment and improving independence in everyday life activities. The study was approved by the Ethics Committee Zurich and registered on ClinicalTrials.gov (NCT02237222).

\section{Participants}

Children and adolescents were eligible for the study if they (1) were between 6-18 years of age; (2) had an acquired or congenital neuromotor diagnosis, such as stroke, traumatic brain injury, or cerebral palsy; (3) were inpatients at the rehabilitation center; and (4) their rehabilitation program contained therapy sessions in which they actively trained their gait function. Therapists reported children who met these inclusion criteria. These children and their parents subsequently received oral and written study information by a research assistant and were consecutively 
recruited between April 2014 and January 2016, provided that at least one parent understood German well. Signed informed consent and assent by the legal guardian and the child had been given. Adhering to the criteria provided by the COSMIN guidelines, we aimed for a sample size of at least $50 .{ }^{17}$

\section{Measures}

Functional Mobility Scale (FMS): For the inpatient situation, we substituted the home $(5 \mathrm{~m})$, school $(50 \mathrm{~m})$, and community $(500 \mathrm{~m})$ settings ${ }^{11}$ into mobility within the patient room, within the rehabilitation center (between ward, therapies, and inpatient school), and outside the rehabilitation center.

For the Functional Assessment Questionnaire - walking scale (FAQ), we considered the children's functional mobility within the rehabilitation center (levels 1-5) or outside the rehabilitation center (levels 6-10). ${ }^{6}$

The 6-minute walking test investigates functional capacity. ${ }^{18}$ Limited to moderate evidence exists for its convergent validity with various body function parameters in children with chronic conditions. Its test-retest reliability in children with $\mathrm{CP}$ is almost perfect, $, 2,19,20$ while evidence for its responsiveness is limited and only available for children with Duchenne Muscular Dystrophy. ${ }^{20}$

The 10-meter walking test evaluates self-selected (10MWTss) or maximal (10MWTm) gait speed over a short distance (Watson 2002). ${ }^{21}$ Relative test-retest reliability of the 10MWT is high in children with neurological gait disorders. ${ }^{2,19}$

The Gross Motor Function Measure-88 (GMFM-88) is a standardized tool to measure gross motor function in children with motor disabilities. ${ }^{22}$ The 24 ordinalscale items of the dimension $E$ assess walking, running and jumping abilities. The psychometric properties of the total GMFM-88 are well explored, especially in children with CP. ${ }^{23}$ Evidence for the psychometric properties of the single dimensions is mostly unknown. Timed walking tests and GMFM E were mainly performed in children for whom these tests were clinically indicated as part of their inpatient rehabilitation program.

The Functional Independence Measure for children (WeeFIM) assesses a child's level of independence during various activities of daily living on the performance 
level (Uniform Data System for Medical Rehabilitation, Buffalo, NY, USA). The items are scored on a 7-point ordinal scale. ICCs of the locomotion subscale are 0.94-0.97 for intra- and $0.73-0.85$ for interrater reliability, and correlations with mobility domains of other functional measures are strong. ${ }^{24,25}$ We used only the walking item of the locomotion subscale.

A 5-point global rating scale (GRS) (somewhat/much worse, unchanged, somewhat/much better), specifically designed for this study, was used as an anchor, quantifying the physiotherapists' perceived change of the children's functional mobility by asking: „compared to the beginning of active gait rehabilitation, how has the child's functional mobility in everyday life changed in your opinion?"

\section{Study procedure}

We used the German versions of both questionnaires, which had been approved by the authors of the original English versions. Details about the translation procedure and results on their reliability and validity are reported elsewhere..$^{10,12}$

Patients' age, gender, diagnosis, and length of rehabilitation stay was collected from their electronic medical records. Measurements took place at two time points: $T_{0}$ at the start and $T_{1}$ at the end of the active gait rehabilitation program. This period usually varies from four weeks to six months.

The physiotherapist who was mainly responsible for the therapy of the child completed the FMS and the FAQ at $T_{0}$ and $T_{1}$, and the GRS at $T_{1}$. As the therapists had no previous experience with the FMS or $F A Q$, they received brief protocolled instructions beforehand. To ensure that the therapists filled in the forms accurately and adhered to the standardized instructions, an independent research fellow (C.A.) performed a standardized interview. Both, instructions and interview were based on the directives found on the questionnaires. To reduce risk of bias, physiotherapists and research fellow were unaware of the other test results and did not have access to $T_{0}$ ratings when scoring $F M S, F A Q$ and $G R S$ at $T_{1}$. Details about the implementation of the other tests are outlined in supplemental Table 5.1. 


\section{Statistical analysis}

Descriptive statistics are presented for relevant characteristics of the study population and outcome measures. SPSS version 24.0 (IBM SPSS Statistics, Armonk, NY, USA) was used for statistical analyses. We treated each FMS distance as a separate measure.

Regarding hypotheses 1) and 2), we calculated Spearman correlation coefficients and $95 \%$ confidence intervals employing bootstrapping, i.e. random sampling with replacement, (bias corrected and accelerated method with 1000 samples) to quantify the relationship between the change scores of the FMS/FAQ, the comparator measures, and the GRS. ${ }^{26}$ We were interested in changes of the construct 'walking capacity' rather than in changes of each single capacity measure. Therefore, we averaged these correlations by transforming each of them into Fisher's $Z$ scores, summarized and averaged these scores, and finally transferred the averaged Fisher's $Z$ scores back into a correlation coefficient. ${ }^{10}$ We interpreted values from 0 0.19 as very weak, $0.2-0.39$ as weak, $0.4-0.59$ as moderate, $0.6-0.79$ as strong, and $\geq 0.8$ as very strong. ${ }^{27}$

To test hypothesis 3, we performed nonparametric ROC curves analyses between the dichotomized GRS and the FMS/FAQ. ${ }^{28}$ We considered a value of the AUC of at least 0.7 as appropriate to demonstrate the FMS/FAQ's ability to discriminate between children who had improved and children who had not improved according to the therapists rating on the GRS. ${ }^{29}$ Regarding hypothesis 4, we used the MannWhitney $U$ test to quantify the differences between the two groups and set the statistical level of significance for a two-tailed test at $\alpha=0.05$.

We calculated the Youden Index (sensitivity + specificity -1) for all points of the ROC curve and selected the maximum value of this index as optimal cut-off point for the MIC of the tools. ${ }^{29}$ Missing data was not replaced.

\section{RESULTS}

The active gait rehabilitation period of the 64 participants ( 6 to 18.5 years old) lasted on average 46 days (SD 41.9 days) and varied between 14 and 295 days. Further 
characteristics of the study population and information on the measurements are presented in Table 5.1 .

Table 5.1. Descriptives of the study population $(n=64)$

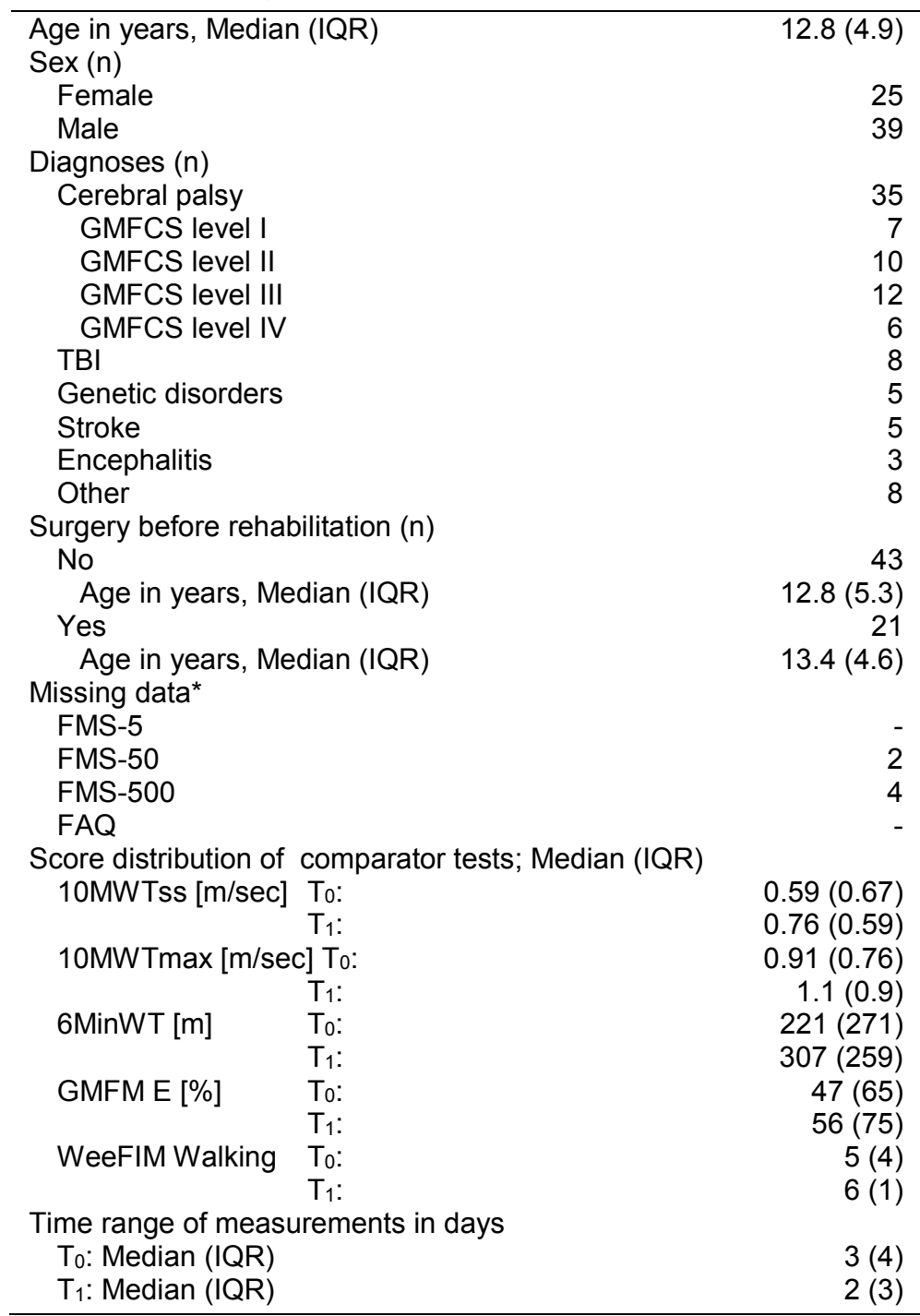

*Missing data can be attributed to children who did not cover these distances in any form at the time of data collection. Abbreviations: IQR, Interquartile range; GMFCS, Gross Motor Function Classification Scale; TBI, Traumatic brain injury; FMS-5/50/500, Functional Mobility Scale 5/50/500 metres; FAQ, Gillette Functional Assessment Questionnaire - walking scale; 10MWTss/m, 10meter walking test selfselected/maximal speed; 6MinWT, 6minute walking test; GMFM E, Gross Motor Function Measure dimension E; WeeFIM walking, Functional Independence Measure for Children walking item; GRS, Global rating scale of therapist's perceived change; $T_{0}$, start of active gait rehabilitation; $T_{1}$, end of active gait rehabilitation. 
The functional abilities of the participants differed widely and ranged over the whole spectrum of the FMS and FAQ (see Figure 5.1.). Thus, not all children were able to perform all capacity tests and/or at both time points (see Table 5.1.).

Change scores varied largely from -2 to 5 for the FMS and from -1 to 7 for the $F A Q$ (Figure 5.1).

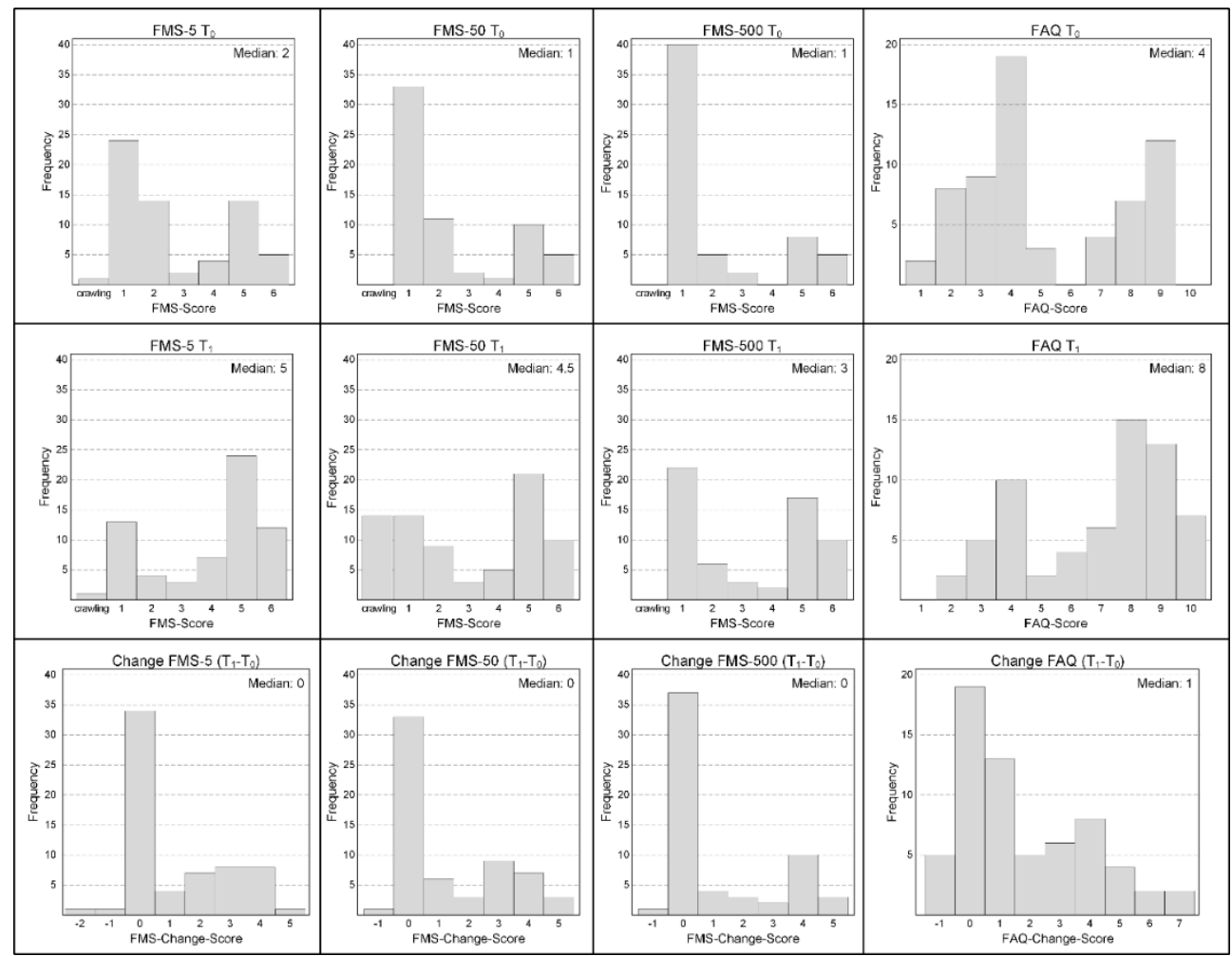

Figure 5.1. Frequency distributions of the Functional Mobility Scale and the Gillette Functional Assessment Questionnaire - walking scale.

FMS-5/50/500, Functional Mobility Scale 5/50/500 meters; FAQ, Gillette Functional Assessment Questionnaire - walking scale; T0, start of active gait rehabilitation; T1, end of active gait rehabilitation

Correlations between the change scores of the FMS/FAQ and the change scores of the comparator tests were all positive. The rs for the comparisons with change scores of the averaged capacity tests were $0.39 / 0.49 / 0.40$ for the FMS-5/50/500 and 0.35 for the FAQ, while those with the WeeFIM walking change scores ranged from 0.54-0.76 (see Table 5.2.a). 
Correlations between the GRS and the change scores of the FMS and FAQ were moderate to strong and ranged from $0.57-0.76$, while the GRS correlated weakly with the change scores of the averaged capacity tests ( $r s=0.36$; see Table 5.2.b).

Table 5.2. Spearman correlations ( $95 \%$ confidence intervals) between the change scores of the Functional Mobility Scale (FMS), Gillette Functional Assessment Questionnaire - walking scale (FAQ) and other gait measures (2.a) and between the global rating scale (GRS) of the therapists' perceived change and the change scores of gait measures (2.b)

Table 5.2.a

\begin{tabular}{lrrrrrr} 
& $\begin{array}{r}\Delta \text { Capacity } \\
\text { Tests }\end{array}$ & $\Delta$ 10MWTss & $\Delta$ 10MWTm & $\Delta 6$ MinWT & $\Delta$ GMFM E & $\begin{array}{r}\Delta \text { WeeFIM } \\
\text { walking }\end{array}$ \\
\hline$\triangle$ FMS-5 & 0.39 & 0.20 & 0.47 & 0.50 & 0.37 & 0.54 \\
& $(-0.06-0.72)$ & $(-0.45-0.72)$ & $(0.14-0.73)$ & $(0.18-0.75)$ & $(-0.08-0.68)$ & $(0.28-0.74)$ \\
N & & 23 & 19 & 20 & 23 & 53 \\
$\triangle$ FMS-50 & 0.49 & 0.38 & 0.70 & 0.36 & 0.45 & 0.73 \\
& $(0.06-0.77)$ & $(-0.02-0.68)$ & $(0.43-0.85)$ & $(-0.14-0.71)$ & $(-0.16-0.79)$ & $(0.58-0.85)$ \\
N & & 23 & 19 & 20 & 23 & 52 \\
$\triangle$ FMS-500 & 0.40 & 0.40 & 0.35 & 0.41 & 0.45 & 0.61 \\
& $(-0.15-0.77)$ & $(-0.08-0.73)$ & $(-0.37-0.77)$ & $(0.00-0.75)$ & $(-0.13-0.80)$ & $(0.39-0.79)$ \\
N & & 22 & 19 & 20 & 23 & 50 \\
$\triangle F A Q$ & 0.35 & 0.19 & 0.42 & 0.44 & 0.36 & 0.76 \\
& $(-0.10-0.70)$ & $(-0.34-0.64)$ & $(-0.09-0.75)$ & $(0.08-0.72)$ & $(-0.06-0.68)$ & $(0.62-0.84)$ \\
N & & 23 & 19 & 20 & 23 & 53 \\
\hline
\end{tabular}

Table 5.2.b

\begin{tabular}{lrrrrr}
$\begin{array}{l}\text { Performance } \\
\text { Measures }\end{array}$ & $\triangle F M S-5$ & $\triangle F M S-50$ & $\triangle F M S-500$ & $\triangle F A Q$ & $\begin{array}{r}\triangle \text { WeeFIM } \\
\text { Walking }\end{array}$ \\
\hline & 0.57 & 0.67 & 0.64 & 0.67 & 0.63
\end{tabular}

\begin{tabular}{lrrrrr} 
& $(0.36-0.73)$ & $(0.48-0.83)$ & $(0.47-0.76)$ & $(0.51-0.79)$ & $(0.43-0.79)$ \\
$\mathrm{N}$ & 63 & 59 & 59 & 63 & 52 \\
\hline Capacity & $\triangle$ Capacity & $\triangle 10 \mathrm{MWTs}$ & $\triangle 10 \mathrm{MWTm}$ & $\triangle 6 \mathrm{MinWT}$ & $\triangle \mathrm{GMFM} \mathrm{E}$ \\
Measures & Tests & & & & \\
\hline & 0.36 & 0.45 & 0.09 & 0.45 & 0.43 \\
$\mathrm{~N}$ & $(-0.13-0.71)$ & $(0.02-0.73)$ & $(-0.48-0.61)$ & $(0.04-0.74)$ & $(-0.06-0.74)$ \\
\hline
\end{tabular}

$\triangle$, change score; 10MWTss/m, 10meter walking test self-selected/maximal speed; 6MinWT, 6minute walking test; GMFM E, Gross Motor Function Measure dimension E; WeeFIM walking, Functional Independence Measure for Children walking item

The ROC curves analyses between the dichotomized GRS (unchanged versus somewhat/much better) and the FMS/FAQ change scores revealed AUC values above the a-priori defined threshold of 0.7 (FMS-5/50/500/FAQ: 0.79/0.75/0.76/0.80; see supplemental Figure 5.1.). No child was rated as somewhat/much worse on the GRS, although six children partially deteriorated in FMS or FAQ scores. 
The median change scores in the non-operated children were 0 for the FMS and 1 for the FAQ. In the post-surgical children, the median change scores were $3 / 3 / 2$ for the FMS 5/50/500 and 3 for the FAQ (see Figure 2). The differences were statistically significant for all change scores between the two groups (FMS-5/50/500/FAQ: Z=3.55/-3.78/-2.82/-3.01; see Figure 5.2.).

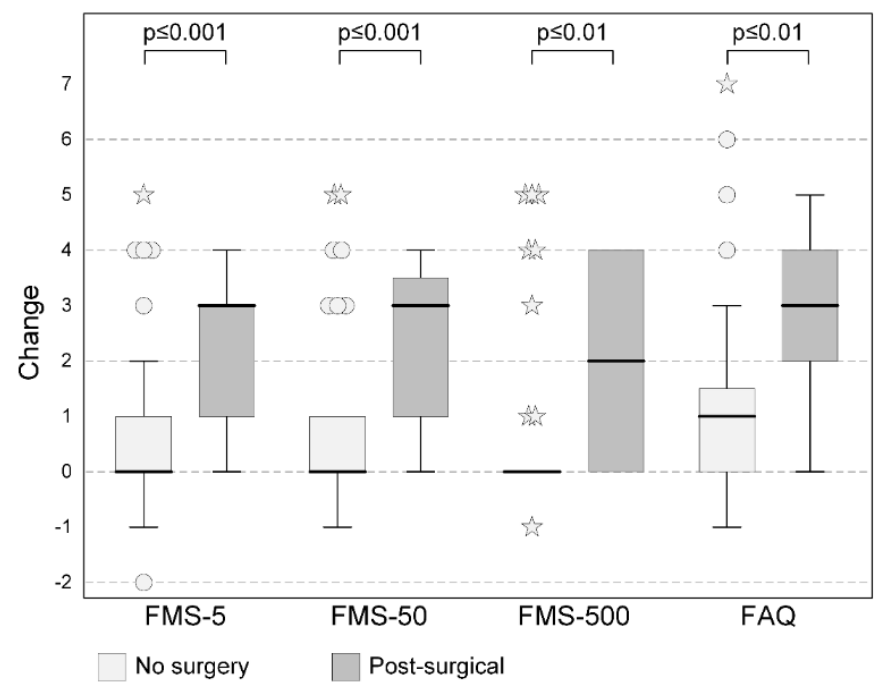

Figure 5.2. Comparison of change scores between children who had received surgery or not prior to undergoing rehabilitation.

FMS-5/50/500, Functional Mobility Scale 5/50/500 meters; FAQ, Gillette Functional Assessment Questionnaire - walking scale; horizontal line, median; box, interquartile range; whiskers, $\pm 1.5^{*}$ interquartile range; small circle, mild outlier $\left(>1.5^{*}\right.$ interquartile range); star, extreme outlier $\left(>2^{*} 1.5\right.$ interquartile range)

The maximum value of the Youden Index resulted in an optimal cut-off point of 0.5 for each distance of the FMS and 1.5 for the FAQ. Thus, in practice, children have to improve at least 1 level on the FMS-5/-50/-500 and 2 levels on the FAQ to achieve the MIC.

\section{DISCUSSION}

We investigated the responsiveness of the FMS and FAQ on a construct-based (correlations) and a criterion-based (ROC curves) method with pre-set hypotheses in children and adolescents with neuromotor disorders in an inpatient 
neurorehabilitation setting. Further, we calculated the MIC using an anchor-based approach for the two measures.

Our results regarding the correlation strengths with the averaged capacity change scores only partly confirmed hypothesis 1 , as only the FMS-50/500 reached the expected level of 0.4 , whereas the correlations were lower for the FMS-5 and FAQ. These results were not in line with previous research in outpatients, which showed strong (positive) correlations between changes in FMS scores and changes in outcomes of other measures of functional activity assessed with questionnaires $\left(r_{s}=0.75-0.83\right)$ and activity monitors $\left(r_{s}=0.78-0.89\right){ }^{5}$

The stronger correlations of the FMS/FAQ with the change score of the WeeFIM walking in our study may reflect that these measures all rate change in performance in everyday situations within the rehabilitation setting, while the capacity measures required the children to perform changes on their maximal level within a standardized test situation.

As information on the responsiveness of the clinically often-used gait comparator measures is largely missing, we designed a GRS to estimate the therapist's perceived change of the children's functional mobility in parallel. Because the reliability of this GRS is unknown, we used it within a construct and criterion approach. ${ }^{9}$ Correlations between the GRS and the FMS/FAQ's change scores were all moderate to strong, showing that the FMS and FAQ could adequately reflect the therapists' judgment regarding the children's functional change in everyday life mobility during inpatient rehabilitation. As the correlation between the GRS and the change score of the averaged capacity tests was only weak, hypothesis 2 was confirmed. We could also confirm hypothesis 3 , in which we used the GRS as external criterion of the children's change in functional mobility. The AUC values ranged between $0.75-0.80$ and showed that both, FMS and FAQ, could be used to discriminate between children whose walking performance remained unchanged versus those who improved.

We additionally compared the mean changes of the FMS and FAQ in subgroups of children who had undergone orthopaedic surgery and the other children, because the first group was expected to improve more than the other group. The first rating 
took place when children just started active gait rehabilitation and were allowed to start loading their legs after orthopaedic surgery. Due to this low initial level of walking ability, we expected the post-surgical children to improve more distinctly compared to the others. Despite that the non-surgical group also comprised children with acute acquired injuries whom we expected to undergo large improvements due to spontaneous neurological recovery in combination with the rehabilitation program, we could confirm hypothesis 4 . Also a previous study on the FMS in the outpatient setting demonstrated longitudinal changes in the outcomes of the scale in children who had received orthopaedic multilevel surgery. ${ }^{5}$

It is important to notice that the potential of improvement largely depends on a child's basic motor abilities. Using Rasch analysis, Stout et al. provided a common scale for different classification systems and outcome measures of physical functioning. With that, they illustrated the probability of successfully performing tasks of different difficulty levels depending on an individual's present motor abilities. ${ }^{30}$ Consequently, a child with a score of 1/1/1 for the FMS-5/50/500 at $T_{0}$ will have more space for improvement than a child entering rehabilitation with a score of $6 / 6 / 6$. In our study, $38 \%$ of the children had a score of 1 over all three distances of the FMS (which decreased to $20 \%$ at the second measurement time point). Although this could be interpreted as a floor effect, it is not of interest as long as the measure is used to evaluate functional mobility regarding a child's walking ability. ${ }^{9} \mathrm{~A}$ ceiling effect was not an issue, as only $8 \%$ of the children had an initial score of 6/6/6 (compared to $17 \%$ at the end). Regarding possible floor and ceiling effects of the FAQ, only $3 \%$ and $0 \%$ of the children had initially the lowest and highest possible score, respectively.

So far, responsiveness has only been evaluated for the FMS. ${ }^{5,14}$ The correlations in our study were lower than previously reported. A reason may be that the other studies took place in the outpatient setting, exclusively looked at children after surgery, and used other comparator instruments. However, we were not able to test this assumption because in our study the sample sizes of specific subgroups were too small to calculate reliable correlations. 
The MIC values indicate a 1-point (FMS) and 2-point increase (FAQ) to be clinically meaningful. These values seem logical from a contextual point, as each level of the FMS signifies the (in)dependence on/from another type of walking device, while the FAQ levels vary unevenly in terms of distance, environment, mobility, and independence. However, as both measures are ordinal-scaled, the MIC may be different for children on the lower and higher end of the scales. Our reported MIC of 2 for the $F A Q$ is consistent with the overlapping item difficulty levels for the $F A Q$ levels $\leq 5$ and 6 derived by Rasch analysis. ${ }^{30}$ Considering the results of the Rasch analysis, an MIC of 1 might be more likely for children with an FAQ level 7 and higher at $T_{0}$. To address these potential differences over the whole range of the scales, and thus enabling an estimation of the MIC for individual patients, larger patient samples would be necessary to allow a differentiation between various motor ability subgroups.

\section{Methodological considerations}

The FMS distances represent children's usual surroundings (home, school, and community), which we had to substitute for the inpatient situation. However, previous research on the interrater reliability between health professionals, who measured inpatient everyday life mobility, and parents, who rated their child's mobility in the usual environments, provide evidence that our adaptations made for the inpatient setting seem to correspond well to the home setting. ${ }^{12}$ In line with the recommendations of de Vet et al., we used an anchor-based approach to assess the MIC, as this includes a definition of what is considered minimally important. ${ }^{9}$ Of course, the rating of the parents or even children could be considered most relevant in the context of MIC. However, because of the inpatient setting (parents are not present throughout the day; for most children it would be difficult to remember the initial situation), the GRS was rated by the therapists. For the same reasons, the therapists scored the FMS and FAQ, in contrast to the measures' original test administration, which involves child- or parent-report. The fact that the GRS was rated by the treating therapist could have resulted in a slight overestimation of the perceived change. While we had to dichotomize the GRS ratings for the ROC analysis at an arbitrary chosen cut-off point, analyses showed that the MICs would 
not change with another selected cut-off level. Unfortunately, we could not compare the MIC with the smallest detectable change (SDC), as the SDC is still unknown for both measures.

Although we exceeded the targeted sample size of 50 , the samples were smaller for the analyses with the capacity tests and the subgroups regarding surgical/nonsurgical intervention. As we aimed for a representative sample of our inpatient rehabilitation setting, it entailed that not every included patient was able to perform every test at both time points. This fact could have biased the reported correlations regarding hypotheses 1 and 2 . However, as the groups with and without capacity test data did not differ systematically, the correlations can be applied to the entire patient sample. The duration between the two rating time points varied considerably, which is an inevitable characteristic in neurorehabilitation. At the same time, the heterogeneity of our sample in terms of age, diagnoses, mobility levels, and rehabilitation duration facilitates the transfer of our results to other inpatient settings.

\section{CONCLUSION}

This is the first study that evaluated the responsiveness of the FMS and FAQ within a pediatric inpatient setting. It showed that both tools are suitable to depict change that has occurred in children's gait performance. Moreover, the results facilitate the interpretation of FMS and FAQ scores in clinical practice and research by defining an MIC for both tools.

\section{ACKNOWLEDGMENTS}

The authors would like to thank all children, adolescents, and parents who participated in this research project. We thank Corina Klöti and the physiotherapists who helped collecting the data. Further, we gratefully acknowledge funding by the Mäxi Foundation, Zurich, Switzerland, for this project. We also acknowledge the Children's Research Center (CRC) of the University Children's Hospital Zurich and the Neuroscience Center Zurich (ZNZ). 


\section{DECLARATION OF INTEREST}

The authors have stated that they had no interests which might be perceived as posing a conflict or bias.

\section{REFERENCES}

1. Zwicker JG, Mayson TA. Effectiveness of Treadmill Training in Children With Motor Impairments : An Overview. Pediatr Phys Ther. 2010;22:361-377.

2. Ammann-Reiffer C, Bastiaenen CHG, de Bie RA, van Hedel HJA. Measurement Properties of Gait-Related Outcomes in Youth With Neuromuscular Diagnosis: A systematic review. Phys Ther. 2014;94(8):1067-1082.

3. Holsbeeke L, Ketelaar M, Schoemaker MM, Gorter JW. Capacity, capability, and performance: different constructs or three of a kind? Arch Phys Med Rehabil. 2009;90(5):849-855.

4. Wilson NC, Chong J, Mackey AH, Stott NS. Reported outcomes of lower limb orthopaedic surgery in children and adolescents with cerebral palsy: a mapping review. Dev Med Child Neurol. 2014;56(9):808-814.

5. Graham HK, Harvey A, Rodda J, Nattrass GR, Pirpiris M. The Functional Mobility Scale (FMS). J Pediatr Orthop. 2004;24(5):514-520.

6. Novacheck TF, Stout JL, Tervo R. Reliability and Validity of the Gillette Functional Assessment Questionnaire as an Outcome Measure in Children with Walking Disabilities. J Pediatr Orthop. 2000;20(1):75-81.

7. Himuro N, Abe H, Nishibu H, Seino T, Mori M. Easy-to-use clinical measures of walking ability in children and adolescents with cerebral palsy: a systematic review. Disabil Rehabil. 2017;39(10):957-968.

8. Mokkink LB, Terwee CB, Patrick DL, et al. The COSMIN study reached international consensus on taxonomy of measurement properties for HRPR outcomes. J Clin Epidemiol. 2010;63:737-745.

9. de Vet HC, Terwee CB, Mokkink LB, Knol DL. Measurement in Medicine: A practical guide. 2011; Cambridge: Cambridge University Press.

10. Ammann-Reiffer C, Bastiaenen CHG, Klöti C, van Hedel HJA. Concurrent Validity of Two Gait Performance Measures in Children with Neuromotor Disorders. Phys Occup Ther Pediatr. 2018;2638:1-12.

11. Harvey AR, Morris ME, Graham HK, Wolfe R, Baker R. Reliability of the functional mobility scale for children with cerebral palsy. Phys Occup Ther Pediatr. 2010;30(2):139-149.

12. Ammann-Reiffer C, Bastiaenen CHG, de Bie RA, van Hedel HJA. Interrater Reliability of Two Gait Performance Measures in Children with Neuromotor 
Disorders Across Two Different Settings. Dev Med Child Neurol. 2017;59(11):11581163.

13. Harvey A, Baker R, Morris ME, Hough J, Hughes M, Graham HK. Does parent report measure performance? A study of the construct validity of the Functional Mobility Scale. Dev Med Child Neurol. 2010;52(2):181-185.

14. Harvey A, Graham HK, Morris ME, Baker R, Wolfe R. The Functional Mobility Scale: ability to detect change following single event multilevel surgery. Dev Med Child Neurol. 2007;49(8):603-607.

15. Stout JL, Gage JR, Schwartz MH, Novacheck TF. Distal femoral extension osteotomy and patellar tendon advancement to treat persistent crouch gait in cerebral palsy. J Bone Jt Surg Am. 2008;90:2470-84.

16. Trost JP, Schwartz MH, Krach LE, Dunn ME, Novacheck TF. Comprehensive short-term outcome assessment of selective dorsal rhizotomy. Dev Med Child Neurol. 2008;50:765-71.

17. Terwee CB, Mokkink LB, Knol DL, Ostelo RWJG, Bouter LM, de Vet HCW. Rating the methodological quality in systematic reviews of studies on measurement properties: a scoring system for the COSMIN checklist. Qual Life Res. 2012;21(4):651-657.

18. American Thoracic Society. ATS Statement: Guidelines for the Six-Minute Walk Test. Am J Respir Crit Care Med. 2002;166:111-117.

19. Graser J V, Letsch C, van Hedel HJA. Reliability of timed walking tests and temporo-spatial gait parameters in youths with neurological gait disorders. BMC Neurol. 2016;16(1):15.

20. Bartels B, de Groot JF, Terwee CB. The Six-Minute Walk Test in Chronic Pediatric Conditions: A Systematic Review of Measurement Properties. Phys Ther. 2013;93(4):529-541.

21. Watson MJ. Refining the Ten-metre Walking Test for Use with Neurologically Impaired People. Physiotherapy. 2002;88(7):386-397.

22. Russell DJ, Rosenbaum PL, Cadman DT, Gowland C, Hardy S, Jarvis S. The gross motor function measure: a means to evaluate the effects of physical therapy. Dev Med Child Neurol. 1989;31:341-352.

23. Harvey A, Robin J, Morris ME, Graham HK, Baker R. A systematic review of measures of activity limitation for children with cerebral palsy. Dev Med Child Neurol. 2008;50(3):190-198.

24. Ottenbacher KJ, Taylor ET, Msall ME, et al. The stability and equivalence reliability of the functional independence measure for children (WeeFIM). Dev Med Child Neurol. 1996;38:907-916. 
25. Ziviani J, Ottenbacher KJ, Shephard K, Foreman S, Astbury W, Ireland P. Concurrent Validity of the Functional Independence Measure for Children (WeeFIM) and the Pediatric Evaluation of Disabilities Inventory in Children with Developmental Disabilities and Acquired Brain Injuries. Phys Occup Ther Pediatr. 2001;21(2):91101.

26. Ruscio J. Constructing Confidence Intervals for Spearman's Rank Correlation with Ordinal Data: A Simulation Study Comparing Analytic and Bootstrap Methods. J Mod Appl Stat Methods. 2008;7:416-34.

27. Chung M. Encyclopedia of Measurement and Statistics. (Salkind NJ, ed.). London: SAGE Publications, Inc.; 2007.

28. Obuchowski NA. Receiver Operating Characteristic Curves and Their Use in Radiology. Radiology 2003;229:3-8.

29. Terwee CB, Bot SDM, de Boer MR, et al. Quality criteria were proposed for measurement properties of health status questionnaires. J Clin Epidemiol. 2007;60(1):34-42.

30. Stout JL, Gorton 3rd GE, Novacheck TF, et al. Rasch analysis of items from two self-report measures of motor function: determination of item difficulty and relationships with children's ability levels. Dev Med Child Neurol. 2012;54(5):443450. 
Supplemental Table 5.1. Details of the test procedures for the comparator instruments

\begin{tabular}{|c|c|c|c|c|}
\hline 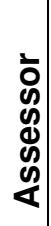 & 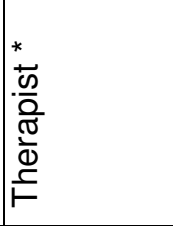 & 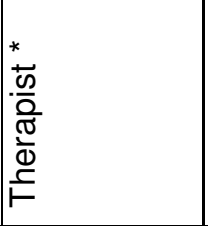 & 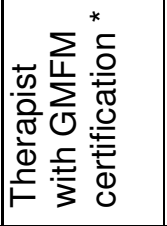 & 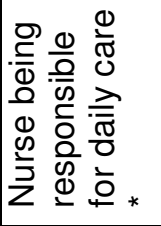 \\
\hline 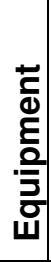 & 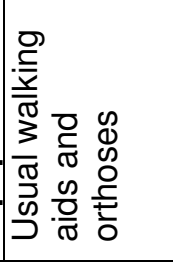 & 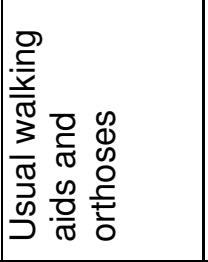 & 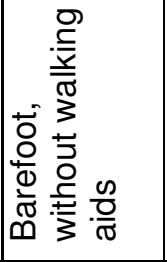 & 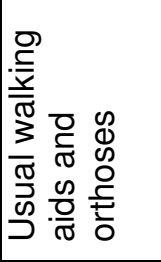 \\
\hline 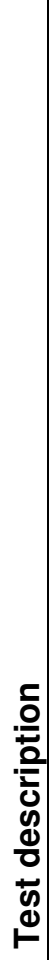 & 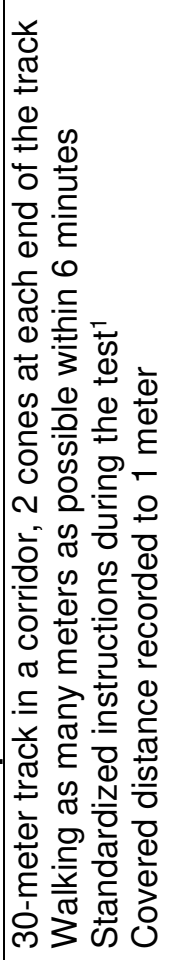 & 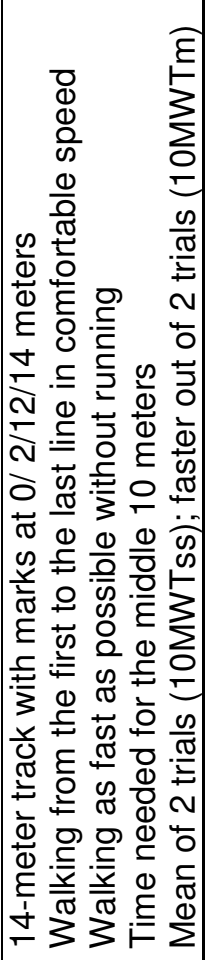 & 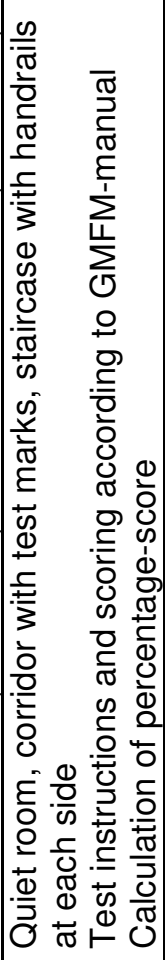 & 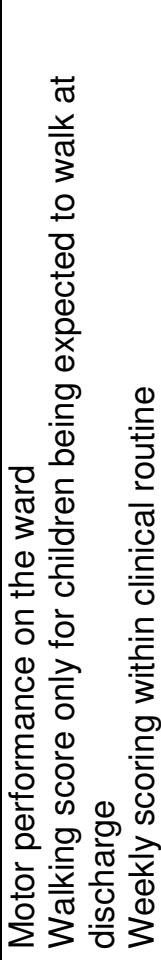 \\
\hline 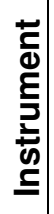 & 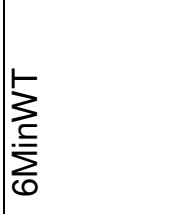 & 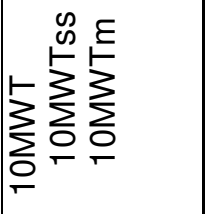 & 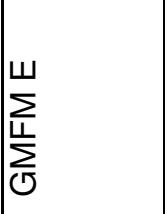 & 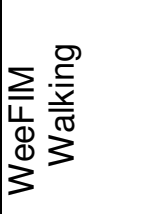 \\
\hline
\end{tabular}


Supplemental Figure 5.1. Receiver operating characteristic (ROC) curves analysis between the dichotomized global rating scale (no change versus improvement) and the change on the Functional Mobility Scale and the Functional Assessment Questionnaire - walking scale

FMS-5/50/500, Functional Mobility Scale 5/50/500 meters; FAQ, Gillette Functional Assessment Questionnaire - walking scale

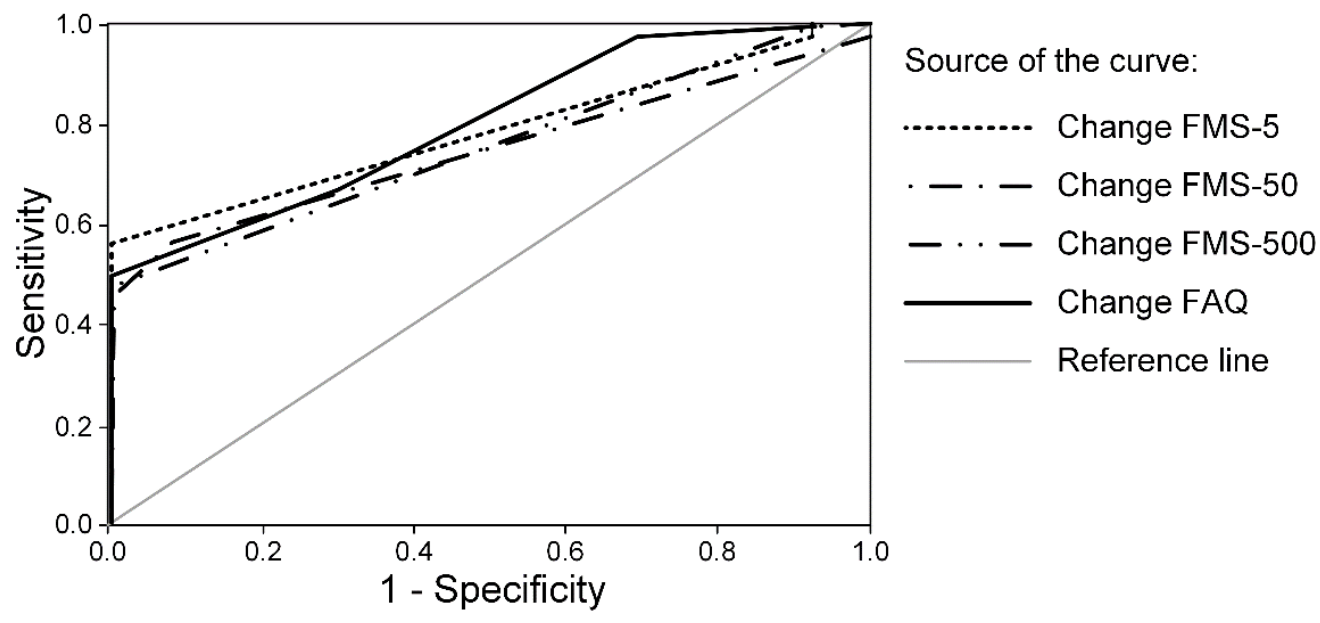



CHAPTER 6

\section{EFFECTIVENESS OF ROBOT-ASSISTED GAIT} TRAINING IN CHILDREN WITH CEREBRAL PALSY: A BICENTER, PRAGMATIC, RANDOMIZED, CROSS-OVER TRIAL (PELOGAIT)

This chapter has been published as:

Ammann-Reiffer C, Bastiaenen CHG, Meyer-Heim AD, van Hedel HJA. Effectiveness of robot-assisted gait training in children with cerebral palsy: A bicenter, pragmatic, randomized, cross-over trial (PeLoGAIT). BMC Pediatr. 2017 Mar 2;17(1):64. 


\section{ABSTRACT}

BACKGROUND: Walking ability is a priority for many children with cerebral palsy (CP) and their parents when considering domains of importance regarding treatment interventions. Partial body-weight supported treadmill training has become an established therapeutic treatment approach to address this demand. Further, new robotic rehabilitation technologies have increasingly been implemented in the clinical setting to allow for longer training sessions with increased step repetitions while maintaining a consistent movement pattern. But the current evidence about its clinical effectiveness in pediatric rehabilitation is weak. The aim of this research project is therefore to investigate the effectiveness of robot-assisted gait training (RAGT) on improvements of functional gait parameters in children with CP.

METHODS/DESIGN: Children aged 6 to 18 years with bilateral spastic CP who are able to walk at least 14 meters with or without walking aids will be recruited in two pediatric therapy centers in Switzerland. Within a pragmatic crossover design with randomized treatment sequences, they perform 5 weeks of RAGT ( 3 times per week with a maximum of 45 minutes walking time each) or a 5-week period of standard treatment, which is individually customized to the needs of the child and usually consists of 1-2 sessions of physiotherapy per week and additional hippotherapy, circuit training as well as occupational therapy as necessary. Both interventions take place in an outpatient setting. The percentage score of the dimension $E$ of the Gross Motor Function Measure-88 (GMFM-88) as primary outcome as well as the dimension D of the GMFM-88, 6-minute and 10-meter walk tests as secondary outcomes are assessed before and at the end of each intervention period. Additionally, a 5-week follow-up assessment is scheduled for the children who are assigned to the standard treatment first. Treatment effects, period effects as well as follow-up effects are analyzed with paired analyses and independent test statistics are used to assess carryover effects.

DISCUSSION: Although RAGT has become an established treatment option to address gait impairments, evidence for its effectiveness is vague. This pragmatic trial will provide important information on its effects under clinical outpatient conditions. 
TRIAL REGISTRATION: ClinicalTrials.gov: NCT00887848. Registered 23 April 2009. 


\section{INTRODUCTION}

Mobility in general as well as the ability to walk is a priority for many children with cerebral palsy (CP) and their parents when considering domains of importance regarding treatment interventions. ${ }^{1}$ Parents of children with CP value walking, especially "correct" walking, as a key component of their children's present and future well-being. ${ }^{2}$ Children with poorer walking abilities report a reduced physical well-being. ${ }^{3}$ Accordingly, acquiring, retaining or improving gait function in these children is often a main goal of the families and the rehabilitation team.

As training intensity, frequency, specificity and level of repetition with variation play a crucial role in promoting sensomotor learning in patients with disorders of the central nervous system, partial body-weight supported treadmill training (PBWSTT) has become an established therapeutic treatment approach. ${ }^{4-6}$ Although two or even three therapists may be needed in severely affected patients to support the movement of the legs and stabilize the pelvis and the trunk, PBWSTT takes an important role in gait rehabilitation of adult patients with a diagnosis of stroke, incomplete spinal cord injury (iSCl), Parkinson's disease (PD) or multiple sclerosis (MS). ${ }^{7-11}$ There is also an increasing body of evidence that PBWSTT improves walking ability, speed and endurance in children, with most evidence being available for children with mild to moderate CP. ${ }^{12-15}$

To allow for longer training sessions with more repetitions while maintaining a consistent movement pattern and reducing the burden of the therapists, new robotic rehabilitation technologies have emerged during the last 15 years and have increasingly been implemented in the clinical setting. ${ }^{16}$ With the addition of virtual reality (VR) scenarios, especially game-based VR, this type of training further offers the patients diversification, fun and challenge. ${ }^{17}$ One domain of rehabilitation robots involves driven gait orthoses (DGO) for robot-assisted gait training (RAGT), which have been originally developed for adults and subsequently adapted for children. ${ }^{18-}$ ${ }^{20}$ One of these DGOs is the Lokomat (Hocoma AG, Volketswil, Switzerland, Figure 6.1.), which, by using the pediatric version, allows a training for children starting at an age of approximately four years. 


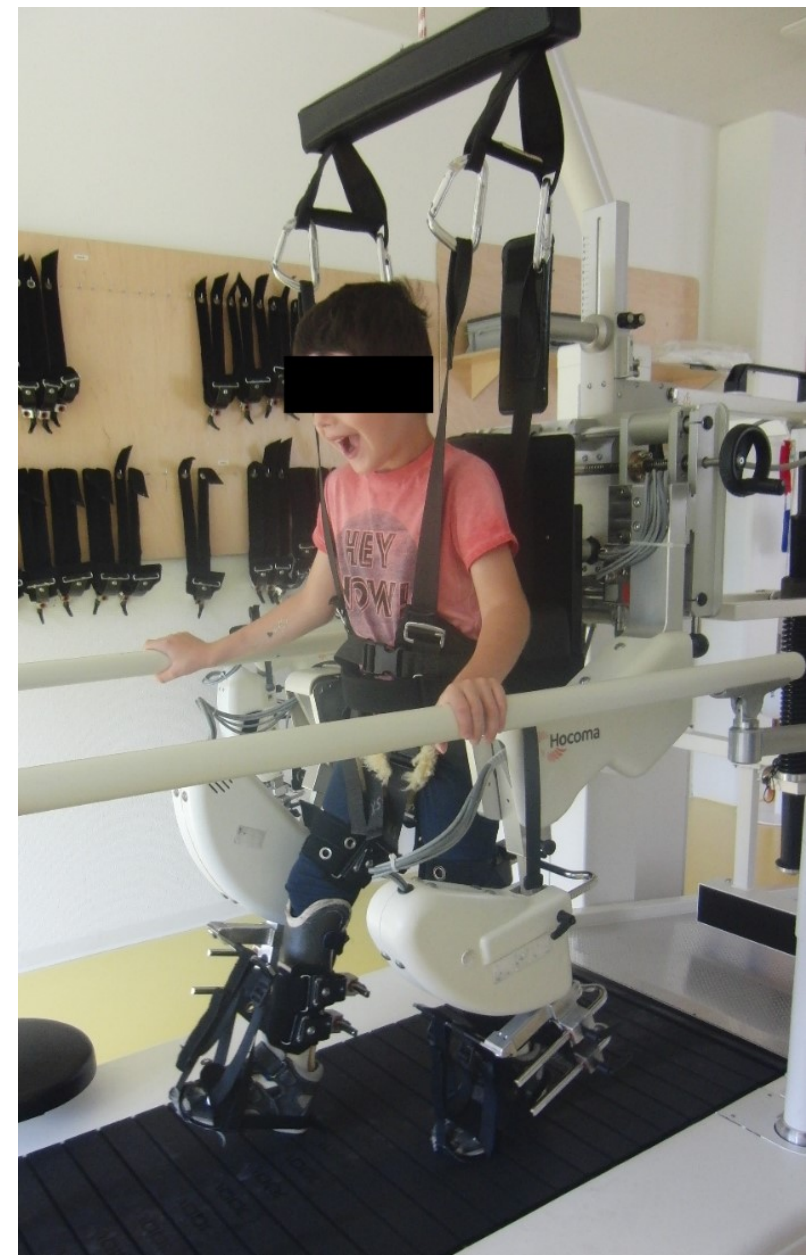

Figure 6.1. Pediatric robot-assisted gait training with the Lokomat.

The Lokomat automates gait therapy on a treadmill by two actuated leg orthoses, which can be individually adapted to the patient's legs and attached with three cuffs, while the patient is secured by means of a counter system with a harness providing partial body-weight support.

RAGT with the Lokomat appears to be effective in improving walking abilities in adult patients with stroke or iSCl. ${ }^{21-23}$ A systematic review confirmed these results and showed limited evidence in patients with MS, traumatic brain injury (TBI) or PD. ${ }^{16}$

In the last years, RAGT has also been tested and implemented in the pediatric neurorehabilitation setting and has been found to be a feasible and safe therapeutic option. ${ }^{20,24}$ However, the current evidence about the clinical effectiveness of RAGT in pediatric populations is weak. Most published results derive from uncontrolled 
single case studies or case series ${ }^{20,25-28}$ and the few published controlled trials ${ }^{29-30}$ provide inconclusive results so far.

The aim of this research project is therefore to investigate the effectiveness of RAGT on improvements of functional gait parameters in ambulatory children with $\mathrm{CP}$ in a pragmatic, randomized, crossover trial.

Our hypothesis is that a 5-week phase of RAGT on the Lokomat in an outpatient setting is superior with regard to the improvement in gait function measured with the dimension E of the Gross Motor Function Measure-88 (GMFM-88) compared to a 5week phase of standard treatment encompassing conventional physiotherapy lessons in children with spastic CP within a crossover design with a randomized treatment sequence.

\section{METHODS/DESIGN}

The design of the study was developed in accordance with the current (2013) version of the Declaration of Helsinki ${ }^{31}$ and the extensions of the CONSORT (Consolidated Standards of Reporting Trials) statement for nonpharmacologic treatment interventions ${ }^{32}$ as well as for pragmatic trials. ${ }^{33}$ Written informed consent and assent is obtained from all the legal guardians and the child by the study coordinator prior to participation.

\section{Design and setting}

This study is designed as a bicenter, single-blinded, pragmatic, randomized crossover trial. It is carried out in the outpatient setting of the rehabilitation center of the University Children's Hospital Zurich in Affoltern am Albis and the Pediatric Therapy Center of the Reha Rheinfelden (Switzerland). Children are randomized to two different pre-specified sequences of interventions. The two interventions are RAGT (T) and usual care (C). A child can be randomized to a T/C sequence (TCgroup) or to a $\mathrm{C} / \mathrm{T} / \mathrm{C}$ sequence (CTC-group).

The duration of study participation varies dependent on group allocation (Figure 6.2.): Children allocated to the TC-group start with a first assessment, followed by a 5-week period of RAGT (T-sequence), a subsequent second assessment, a 5-week period of usual care (C-sequence) and a third assessment. Individual study 
participation will accordingly last 11 weeks for children in the TC-group with three assessment time points.

In contrast to that, children allocated to the CTC-group start with an assessment followed by five weeks of usual care (C-sequence) and a subsequent second assessment. This is succeeded by a 5-week period of RAGT (T-sequence), a third assessment, another 5-week period of usual care (C-sequence) and a follow-up assessment. Thus, study participation for children assigned to the CTC-group lasts 16 weeks. The rationale for the differences in study duration and number of assessments between the study groups is that we want to reduce the burden for the children and their families as much as possible while still gaining information on the preservation of a potential effect at follow-up.

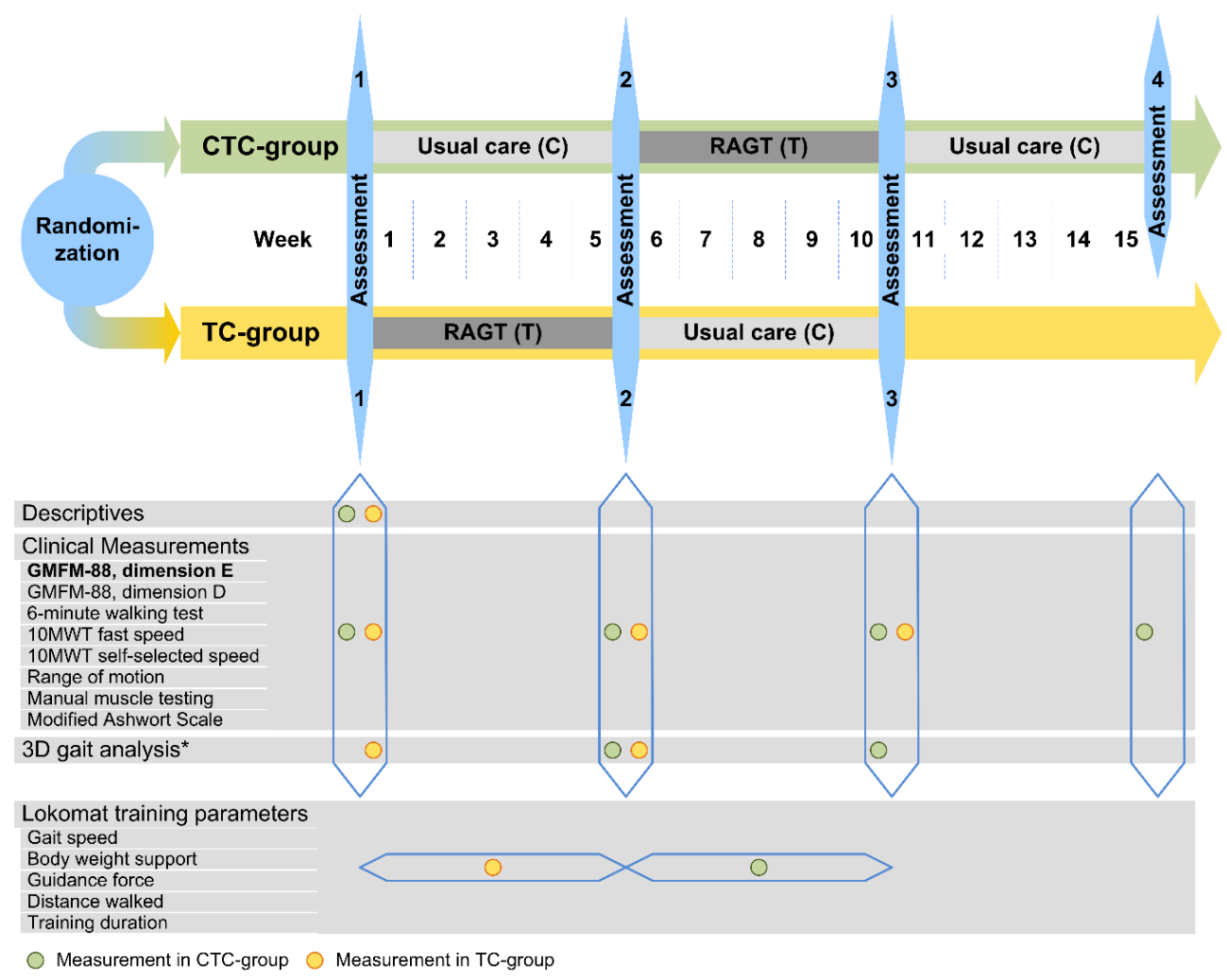

Figure 6.2. Overview of the outcome measures and the measurement time points per group.

RAGT, Robot-assisted gait training; GMFM-88, Gross Motor Function Measure-88; 10MWT, 10-meter walk test; *on an optional basis. 


\section{Eligibility criteria}

Children aged 6 to 18 years with the diagnosis of a bilateral di- or quadriplegic spastic CP and a Gross Motor Function Classification System (GMFCS) level II-IV, who are able to walk at least 14 meters with or without walking aids, are eligible for study participation. Although we are aware that these criteria encompass a wide range of different motor abilities and developmental states, we consider it important that our study population represents the whole range we encounter in clinical practice. Further, children have to be able to follow instructions and communicate pain or discomfort.

Exclusion criteria are neurosurgery or orthopaedic surgery on the lower extremity or trunk within the last six months, having participated in another Lokomat training regime within the previous six months as well as a change in concomitant treatment within the last four weeks before or during the study period. This holds true for all contraindications outlined in the Lokomat manufacturer's manual, as for example severe contractures (i.e. $>20^{\circ}$ knee extension deficit, $>40^{\circ}$ hip extension deficit), bone fractures, open skin lesions or circulatory problems. Further criteria and their clinical implementation are described in a recently published paper by a Lokomat expert panel. ${ }^{34}$

\section{Recruitment}

Patients are recruited by announcement in schools for children with special needs, in pediatric clinics and in outpatient pediatric physical therapy practices. Additionally, we will inform parents of children who have previously trained on the Lokomat and who fulfill the inclusion criteria about the study in a letter. Information about the study is also made available on the website of the two participating centers as well as of the association of Swiss pediatric physiotherapists. Families interested in participating can then take the initiative and contact one of the two rehabilitation centers.

\section{Randomization, allocation concealment, and blinding}

Randomization into the two groups with different intervention sequences is performed using a minimization method with a random factor of 0.9 , including the factors severity of impairment (GMFCS-level II or III/IV), age (6-11 years or 11- 
18years) and Botulinum Toxin A-treatment (present or absent) in the preceding 6 months. Minimization facilitates balancing even small groups in terms of selected patient factors at all stages of a trial. To ensure that group allocation is concealed for patients and their families as well as everyone who is involved at any stage of the trial, the randomization list is generated by a person not belonging to the study team on www.randomizer.at and stored off site. Directly after inclusion of the patient the study coordinator seeks information about group allocation through a phone call or an e-mail. As soon as a child is allocated to one of the two groups, the assessments and trainings are planned according to the assigned intervention sequence, taking into account as much as possible the families' requests and the school schedule of the child.

The rater of the primary outcome and parts of the secondary outcomes is blinded as the performance of the child in these tests is video recorded and scored by means of these videos with the rater being unaware of the date the video has been recorded. The same applies to the statistician.

\section{Intervention}

RAGT (T-sequence): Protocols for PBWSTT as well as RAGT in children are very heterogeneous so far. Session frequencies range from two to five times per week, session durations vary with most studies reporting 20 to 30 minutes of treadmill walking per session, and length of treatment varies from two weeks to five months. ${ }^{34}$ As our training protocol prescribes the Lokomat training on an outpatient basis, we consider a session frequency of three times per week with a maximum of 45 minutes of effective walking time on the Lokomat as feasible and a treatment duration of five weeks as clinically adequate.

Trained and experienced therapists, who are used to working with children, perform the Lokomat trainings. The trainings are executed following recently published recommendations. ${ }^{34}$ Serious games and other available motivating strategies are used in order to increase the child's adherence to the training. Body-weight support, gait speed as well as guidance force of the Lokomat are individually adjusted according to the child's abilities and modified within training sessions and during the course over time. To ensure that uniform standards are applied in both centers, a 
joint practical training session has been performed and the clinical training standards have been discussed beforehand.

Standard therapies can be continued during the intervention period, but should not include specific walking training and not be changed in frequency. Parents list the type as well as the dosage of concomitant therapy in a personal logbook.

Usual care (C-sequence): Usual care normally comprises 1-2 sessions of physiotherapy per week. Hippotherapy, circuit training, as well as occupational therapy can also be elements of standard treatment. These therapies, which are individually customized to the needs of the child, are often implemented in the school setting. The content of the usual care treatment does not specifically address gait training, but rather consists of elements addressing range of motion, tone reduction, balance, activities of daily living, etc. Information about the type and dosage of all therapies is documented in a personal logbook by the parents. As the treatment in our study should represent usual care in clinical practice, we refrained from increasing the dosage for the usual care treatment to a comparable level as for the RAGT intervention.

\section{Descriptive parameters and measures describing the interventions}

An overview of all outcome measures and measurement time points is provided in Figure 6.2.

To make sure that the therapists from both centers apply common standards, all performed measures have been thoroughly discussed and trained within and between the teams.

Descriptive and clinical data like diagnosis, walking aids, undergone operations, age, height and weight are recorded in order to describe the characteristics of the included patients. A description and quantification of the RAGT sessions is provided by various measures including the percentage of body weight support, gait speed, guidance force, distance walked and the number and duration of training sessions.

\section{Primary outcome measure}

The primary outcome measure is the percentage score of the dimension $E$ (walking, running, jumping) of the Gross Motor Function Measure-88 (GMFM-88). It assesses 
the gross motor function with regard to walking ability. ${ }^{35}$ The GMFM is a wellvalidated measurement tool for children with $\mathrm{CP}$, which can be regarded as a gold standard for the assessment of gross motor function in children with $\mathrm{CP}$. The dimension $E$ consists of 24 items, each of which is scored on a 4-point ordinal scale. The children perform the test barefoot, or with shoes and orthoses, if orthoses are used when walking indoors in everyday life. The test is performed without walking aids, regardless of the children's GMFCS-level. A GMFM-certified therapist instructs the children while the test is simultaneously videotaped. These videos are later scored by a blinded rater, who is also a GMFM-certified therapist. The psychometric properties of the total GMFM-88 are well explored in children with CP. Nevertheless, information on single dimensions is rather sparse. There is limited positive evidence for the responsiveness of dimension $\mathrm{E}$, while evidence on reliability is still unknown because of too little patients included in the reliability studies ${ }^{36} \mathrm{~A}$ recently published study showed that video rating is a reliable option for the dimensions $E$ as well as $D$ with ICCs of 0.99 and 0.97 for the agreement of life and video scores. ${ }^{37}$

\section{Secondary outcome measures}

GMFM dimension $D$ : The percentage score of the dimension $D$ (standing) of the GMFM-88 to assess the gross motor function with regard to standing ability. The 13 items are carried out and video rated in the same way as described for the dimension E. As for dimension E, there is evidence for responsiveness of dimension D. ${ }^{35}$ Reliability seems to be high, but sample sizes of these studies were rather small to allow definite conclusions. ${ }^{35,38}$

Six-minute walking test: The 6-minute walk test (6MinWT) is performed on a 30 meter long corridor with poles at each end. The instruction and encouragement of the child follows a standardized test protocol and the covered distance is noted to one meter. ${ }^{39}$ Evidence for the test-retest reliability of the 6MinWT is moderate; its responsiveness has not been established in children with CP so far. ${ }^{36}$

Ten-meter walking test: Gait speed is assessed with the 10-meter walk test (10MWT), which is performed on a 14-meter long track with the child using the walking aid usually used in daily life. The time needed for the middle 10 meters is measured with a stopwatch, allowing 2 meters for acceleration and deceleration 
each. The test is performed twice without a break. For the evaluation of maximal speed (10MWTm), the child is instructed to walk as fast as possible but without running, from the first to the last line. The faster out of two trials is used for further analysis. For self-selected speed (10MWTss) the child is instructed to walk in a normal, comfortable speed from the first to the last line and the mean of two trials is calculated. All gait tests are performed with orthoses and walking aids usually used in daily life. Evidence for test-retest reliability of the 10MWTm is moderate in children with CP. ${ }^{36}$ To our knowledge, responsiveness of the $10 \mathrm{MWm}$ as well as psychometric properties of the 10MWTss have not yet been established in children. Measures of body function: Regarding the child's body function level, range of motion, modified Ashworth scores, and muscle strength by means of manual muscle testing are evaluated in flexion and extension of the hip, knee and ankle joints. ${ }^{40-41}$ These measures will be used for descriptive purposes only.

One complete assessment block takes maximally 120 minutes.

Additionally, children perform a 3-dimensional gait analysis (3DGA) on an optional basis before and at the end of the intervention period to capture spatio-temporal and kinematic data of the lower body during walking. The Helen Hayes marker set with 16 markers for the lower body, seven infrared Vicon MX cameras (Oxford Metrix Ltd., Oxford, UK) and two floor-mounted force platforms (AMTI OR 6-7-2000, Advanced Mechanical Technology Inc., Watertown, MA, USA) are used for the 3DGA. The mean of six clean force plate strikes for each foot and each parameter of interest is calculated for further analysis to obtain a reliability level of at least 0.9 for each evaluated discrete gait parameter irrespective of the child's GMFCS level. ${ }^{42}$

\section{Sample size and power calculation}

A sample size calculation for a $2 \times 2$ crossover design was performed. It indicated a sample size of 30 to be sufficient to detect a difference of $3.7 \%$-points in the dimension E-score of the GMFM- $88,{ }^{43}$ assuming a standard deviation of $6.8 \%$ points, ${ }^{25}$ a power of $80 \%$ and a significance level of $5 \%$. The $3.7 \%$-points represented the cut-off value that best differentiated between great and not great improvement in motor function in children with CP as judged by their therapists. ${ }^{43}$ 
This number has been increased to 34 to allow for a predicted dropout rate of about $10 \%$.

\section{Statistical analysis}

All data are electronically filed by the PI using EpiData software with double data entry and range checks for data values (EpiData Association, Odense, Denmark). The statistical analyses are carried out with SPSS24 (IBM Corporation, New York, USA) and datasets of all study participants are analyzed on an intention-to-treat basis. Descriptive parameters of the participants will be presented. Treatment effects, period effects as well as follow-up effects of the two different treatment interventions are analyzed with paired t-tests or Wilcoxon signed-rank tests for paired samples, with the treatment effect being our primary end-point. Unpaired twosample t-tests or Wilcoxon rank sum tests for unpaired samples are used to determine whether carryover effects are present. ${ }^{44}$ Ninety-five $\%$ confidence intervals are presented with a two-tailed level of significance set at $p<0.05$. The applied analyses are schematically listed in Figure 6.3.

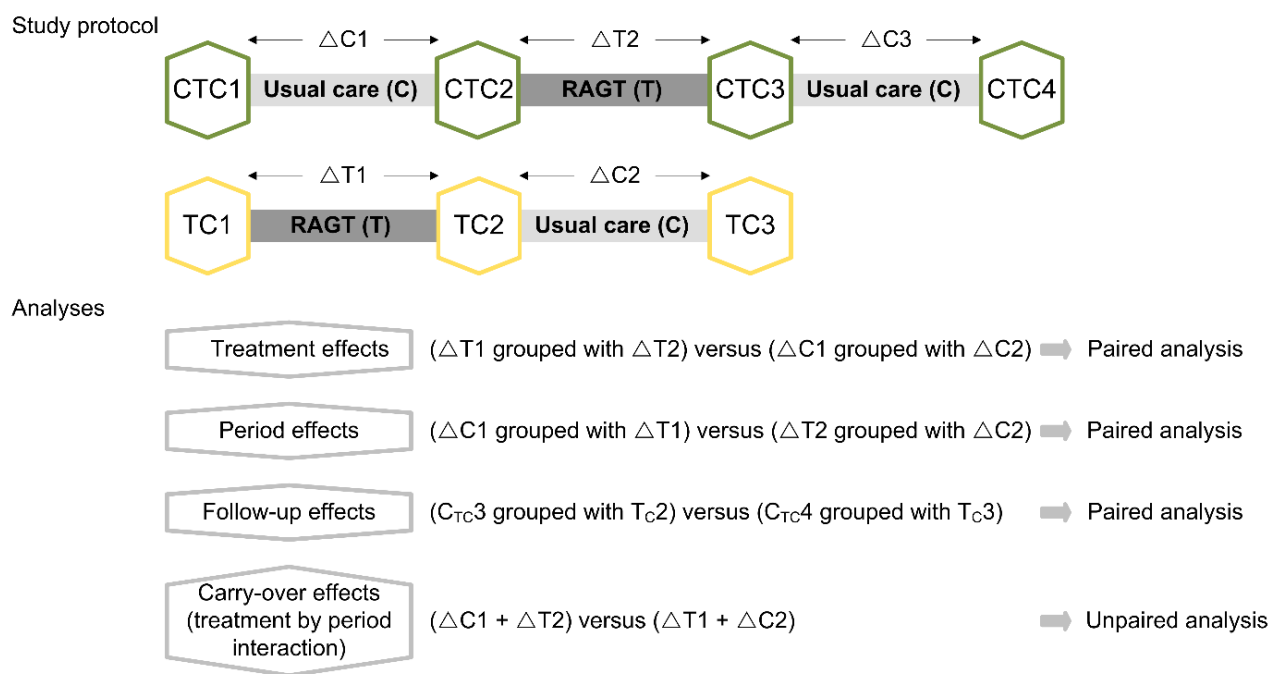

Figure 6.3. Overview of the study protocol and the statistical analyses.

CTC1, baseline assessment in CTC-group; TC1, baseline assessment in TC-group; CTC2, intermediate assessment in CTC-group; TC2, intermediate assessment in TC-group; CTC3, end assessment in CTCgroup; TC3, end assessment in TC-group; CTC4, follow-up assessment in CTC-group; $\triangle \mathrm{C} 1$, change during usual care in CTC-group; $\triangle \mathrm{C} 2$, change during usual care in TC-group; $\triangle \mathrm{C}$ 3, change during followup in CTC-group; $\triangle T 1$, change during robot-assisted gait training in TC-group; $\triangle T 2$, change during robotassisted gait training in CTC-group. 
Subgroup analyses are performed according to impairment level (GMFCS level II vs. GMFCS levels III and IV) and treatment adherence (12 or more training sessions completed vs. less than 12 training sessions), given sufficient numbers within the subgroups.

Missing data will be handled with multiple imputation procedures. ${ }^{45}$

\section{DISCUSSION}

RAGT was implemented in the clinical setting of the two participating pediatric neurorehabilitation centers as innovative therapeutic approach almost 10 years ago. It has become an established treatment option in these clinics to address gait impairments in the in- as well as outpatient setting despite the fact that evidence for its effectiveness is vague. As we want to determine the effects of RAGT as it is applied in the clinical setting, we designed this pragmatic trial. The pragmatic trial design allows to evaluate the effects of an intervention under the usual conditions in which it is applied, whereas explanatory trials determine the effects of an intervention under ideal circumstances. ${ }^{46}$ Thorpe et al. defined a pragmatic-explanatory continuum, an instrument to determine the extent to which a trial can be viewed as pragmatic or explanatory. ${ }^{46}$ Our trial addresses most domains at the rather pragmatic end of this continuum. We make concessions towards a more explanatory approach in two domains: Regarding the flexibility of the experimental design, we standardize frequency, timing and duration of the RAGT intervention and impose some restrictions regarding the co-interventions. A further domain concerns the primary outcome assessment: While we use the dimension E of the GMFM-88, which is a standard outcome in our clinics as well as for the assessment of gross motor functions in children with $\mathrm{CP}$, we deviate from a clear pragmatic approach by videotaping the assessments and including an additional follow-up assessment. We are confident that the chosen trial design allows the evaluation of the effects of RAGT as it is implemented in the daily routine of our clinics, while keeping the additional burden for the participating children and their families as low as possible. This is also the reason why we perform the follow-up assessment only in one group. As participation in this trial still requires a considerable amount of time, we expect some 
difficulties in recruitment. The study is therefore carried out in two centers, namely the only two centers in Switzerland that offer a pediatric Lokomat training on a regular basis so far. In addition, we designed the study as a crossover trial. The strength of this design is that each child receives both treatments, but in a random order. Thus, each patient can act as his or her own control requiring only half the number of patients needed in a parallel group design. As variation within a person is usually less than between patients the treatment effect can be estimated with higher precision despite smaller sample sizes. ${ }^{44}$ On the other hand, a disadvantage of the crossover design is the risk of possible carryover effects, which means that the effect of the treatment in the first period has an effect that lasts into the second period. A carryover effect leads to an underestimation of the effect of the treatment in the second period. By introducing a washout period between the different treatment periods, this risk could be reduced. Introducing a washout period would lead to an additional assessment. In order to reduce the load of the participants to a minimum we abstained from a washout period being aware that this might influence our results.

Although the 5-week follow-up period is rather short, we opted for this time frame because of the increasing risk of confounding factors that is accompanied by a long time interval due to growth, new therapy approaches, illness, operations, increased school load etc.

\section{ACKNOWLEDGMENTS}

We would like to thank the physiotherapists at the rehabilitation center of the Children's University Hospital Zurich in Affoltern am Albis and the Pediatric Therapy Center of the Reha Rheinfelden for their help regarding the execution of assessments and trainings. We further acknowledge the Zurich Center for Neuroscience (ZNZ).

\section{FUNDING}

This trial is partly funded by by the Mäxi-Foundation (Zurich, Switzerland), the Donation UBS AG (by order of a client) and the Stiftung Cerebral (Switzerland). 


\section{COMPETING INTERESTS}

The authors declare that they have no competing interests.

\section{ETHICS APPROVAL AND CONSENT TO PARTICIPATE}

Ethical approval for this study has been obtained from the Ethics Committee Zurich and the Ethics Committee northwest/central Switzerland EKNZ, Switzerland (Reference Number StV-07/07). All participants will be provided with verbal and written information about the study and written informed consent will be obtained prior to enrolment into the study.

\section{REFERENCES}

1. Vargus-Adams JN, Martin LK. Domains of importance for parents, medical professionals and youth with cerebral palsy considering treatment outcomes. Child Care Health Dev. 2011;37(2):276-81.

2. Beveridge B, Feltracco D, Struyf J, Strauss E, Dang S, Phelan S et al. "You gotta try it all": Parents' Experiences with Robotic Gait Training for their Children with Cerebral Palsy. Phys Occup Ther Pediatr. 2014.

3. Dickinson HO, Parkinson KN, Ravens-Sieberer U, Schirripa G, Thyen U, Arnaud $C$ et al. Self-reported quality of life of 8-12-year-old children with cerebral palsy: a cross-sectional European study. Lancet. 2007;369(9580):2171-8.

4.Dobkin BH. Clinical practice. Rehabilitation after stroke. $N$ Engl $J$ Med. 2005;352(16):1677-84.

5. Johansson BB. Brain plasticity and stroke rehabilitation. The Willis lecture. Stroke. 2000;31(1):223-30.

6. Barbeau H, Visintin M. Optimal outcomes obtained with body-weight support combined with treadmill training in stroke subjects. Arch Phys Med Rehabil. 2003;84(10):1458-65.

7. Kwakkel G, Kollen BJ, Wagenaar RC. Long term effects of intensity of upper and lower limb training after stroke: a randomised trial. J Neurol Neurosurg Psychiatry. 2002;72(4):473-9.

8. Moseley AM, Stark A, Cameron ID, Pollock A. Treadmill training and body weight support for walking after stroke. Cochrane Database Syst Rev. 2005(4):CD002840.

9. Morawietz C, Moffat F. Effects of locomotor training after incomplete spinal cord injury: a systematic review. Arch Phys Med Rehabil. 2013;94(11):2297-308. 
10. Ganesan M, Sathyaprabha TN, Pal PK, Gupta A. Partial Body weight support treadmill training in patients with Parkinson's disease: Impact on gait and clinical manifestation. Arch Phys Med Rehabil. 2015;Sep;96(9):1557-65.

11. Swinnen E, Beckwee D, Pinte D, Meeusen R, Baeyens JP, Kerckhofs E. Treadmill training in multiple sclerosis: can body weight support or robot assistance provide added value? A systematic review. Mult Scler Int. 2012;2012:240274.

12. Schindl MR, Forstner $\mathrm{C}$, Kern $\mathrm{H}$, Hesse $\mathrm{S}$. Treadmill training with partial body weight support in nonambulatory patients with cerebral palsy. Arch Phys Med Rehabil. 2000;81(3):301-6.

13. Dodd KJ, Foley S. Partial body-weight-supported treadmill training can improve walking in children with cerebral palsy: a clinical controlled trial. Dev Med Child Neurol. 2007;49(2):101-5.

14. Cherng RJ, Liu CF, Lau TW, Hong RB. Effect of treadmill training with body weight support on gait and gross motor function in children with spastic cerebral palsy. Am J Phys Med Rehabil. 2007;86(7):548-55.

15. Mattern-Baxter K, McNeil S, Mansoor JK. Effects of home-based locomotor treadmill training on gross motor function in young children with cerebral palsy: a quasi-randomized controlled trial. Arch Phys Med Rehabil. 2013;94(11):2061-7.

16. Tefertiller C, Pharo B, Evans N, Winchester P. Efficacy of rehabilitation robotics for walking training in neurological disorders: a review. J Rehabil Res Dev. 2011;48(4):387-416.

17. Labruyere R, Gerber CN, Birrer-Brutsch K, Meyer-Heim A, van Hedel HJ. Requirements for and impact of a serious game for neuro-pediatric robot-assisted gait training. Res Dev Disabil. 2013;34(11):3906-15.

18. Colombo G, Joerg M, Schreier R, Dietz V. Treadmill training of paraplegic patients using a robotic orthosis. J Rehabil Res Dev. 2000;37(6):693-700.

19. Hesse S, Schmidt $H$, Werner $C$, Bardeleben $A$. Upper and lower extremity robotic devices for rehabilitation and for studying motor control. Curr Opin Neurol. 2003;16(6):705-10.

20. Meyer-Heim A, Borggraefe I, Ammann-Reiffer C, Berweck S, Sennhauser FH, Colombo $\mathrm{G}$ et al. Feasibility of robotic-assisted locomotor training in children with central gait impairment. Dev Med Child Neurol. 2007;49(12):900-6.

21. Mayr A, Kofler M, Quirbach E, Matzak H, Frohlich K, Saltuari L. Prospective, blinded, randomized crossover study of gait rehabilitation in stroke patients using the Lokomat gait orthosis. Neurorehabil Neural Repair. 2007;21(4):307-14.

22. Husemann B, Muller F, Krewer C, Heller S, Koenig E. Effects of locomotion training with assistance of a robot-driven gait orthosis in hemiparetic patients after stroke: a randomized controlled pilot study. Stroke. 2007;38(2):349-54. 
23. Wirz M, Zemon DH, Rupp R, Scheel A, Colombo G, Dietz V et al. Effectiveness of automated locomotor training in patients with chronic incomplete spinal cord injury: a multicenter trial. Arch Phys Med Rehabil. 2005;86(4):672-80.

24. Borggraefe I, Klaiber M, Schuler T, Warken B, Schroeder SA, Heinen F et al. Safety of robotic-assisted treadmill therapy in children and adolescents with gait impairment: a bi-centre survey. Dev Neurorehabil. 2010;13(2):114-9.

25. Meyer-Heim A, Ammann-Reiffer C, Schmartz A, Schafer J, Sennhauser FH, Heinen $\mathrm{F}$ et al. Improvement of walking abilities after robotic-assisted locomotion training in children with cerebral palsy. Arch Dis Child. 2009;94(8):615-20.

26. Borggraefe I, Meyer-Heim A, Kumar A, Schaefer JS, Berweck S, Heinen F. Improved gait parameters after robotic-assisted locomotor treadmill therapy in a 6year-old child with cerebral palsy. Mov Disord. 2008;23(2):280-3.

27. Borggraefe I, Kiwull L, Schaefer JS, Koerte I, Blaschek A, Meyer-Heim A et al. Sustainability of motor performance after robotic-assisted treadmill therapy in children: an open, non-randomized baseline-treatment study. Eur J Phys Rehabil Med. 2010;46(2):125-31.

28. Borggraefe I, Schaefer JS, Klaiber M, Dabrowski E, Ammann-Reiffer C, Knecht $B$ et al. Robotic-assisted treadmill therapy improves walking and standing performance in children and adolescents with cerebral palsy. Eur J Paediatr Neurol. 2010;14(6):496-502.

29. Schroeder AS, Homburg M, Warken B, Auffermann H, Koerte I, Berweck S et al. Prospective controlled cohort study to evaluate changes of function, activity and participation in patients with bilateral spastic cerebral palsy after Robot-enhanced repetitive treadmill therapy. Eur J Paediatr Neurol. 2014;18(4):502-10.

30. Druzbicki M, Rusek W, Snela S, Dudek J, Szczepanik M, Zak E et al. Functional effects of robotic-assisted locomotor treadmill thearapy in children with cerebral palsy. J Rehabil Med. 2013;45(4):358-63.

31. World Medical Association Declaration of Helsinki: ethical principles for medical research involving human subjects. JAMA. 2013;310(20):2191-4.

32. Boutron I, Moher D, Altman DG, Schulz KF, Ravaud P. Extending the CONSORT statement to randomized trials of nonpharmacologic treatment: explanation and elaboration. Ann Intern Med. 2008;148(4):295-309.

33. Zwarenstein M, Treweek S, Gagnier JJ, Altman DG, Tunis S, Haynes B et al. Improving the reporting of pragmatic trials: an extension of the CONSORT statement. BMJ. 2008;337:a2390.

34. Aurich-Schuler A, Warken, B., Graser, J.V., Ulrich, T., Borggraefe, I., Heinen, F., van Hedel, H.J.A., Meyer-Heim, A.D. Practical recommendations for robot-assisted treadmill therapy (Lokomat) in children with cerebral palsy: Indications, goal setting, 
and clinical implementation within the WHO-ICF framework. Neuropediatrics. 2015;46(4):248-60.

35. Russell DJ, Rosenbaum PL, Cadman DT, Gowland C, Hardy S, Jarvis S. The gross motor function measure: a means to evaluate the effects of physical therapy. Dev Med Child Neurol. 1989;31(3):341-52.

36. Ammann-Reiffer $\mathrm{C}$, Bastiaenen $\mathrm{CH}$, de Bie RA, van Hedel HJ. Measurement properties of gait-related outcomes in youth with neuromuscular diagnoses: a systematic review. Phys Ther. 2014;94(8):1067-82.

37. Franki I, Van den Broeck C, De Cat J, Molenaers G, Vanderstraeten G, Desloovere K. A study of whether video scoring is a reliable option for blinded scoring of the Gross Motor Function Measure-88. Clin Rehabil. 2014.

38. Bjornson K, Graubert C, McLaughlin J. Test-retest reliability of the gross motor function measure in children with cerebral palsy. Pediatr Phys Ther. 2000;12(4):2002.

39. ATS statement: guidelines for the six-minute walk test. Am J Respir Crit Care Med. 2002;166(1):111-7.

40. Fosang AL, Galea MP, McCoy AT, Reddihough DS, Story I. Measures of muscle and joint performance in the lower limb of children with cerebral palsy. Dev Med Child Neurol. 2003;45(10):664-70.

41. Hislop Helen J. MJ. Danie/s' and Worthinghams Muskeltests - Manuelle Untersuchungstechniken. 7 ed. München - Jena: Urban und Fischer Verlag; 1999.

42. Redekop S, Andrysek J, Wright V. Single-session reliability of discrete gait parameters in ambulatory children with cerebral palsy based on GMFCS level. Gait Posture. 2008;28(4):627-33.

43. Wang HY, Yang YH. Evaluating the responsiveness of 2 versions of the gross motor function measure for children with cerebral palsy. Arch Phys Med Rehabil. 2006;87(1):51-6.

44. Senn S. Cross-over Trials in Clinical Research. 2nd ed. Chichester, West Sussex, UK: John Wiley \& Sons Ltd; 2002.

45. Dziura JD, Post LA, Zhao Q, Fu Z, Peduzzi P. Strategies for dealing with missing data in clinical trials: from design to analysis. Yale J Biol Med. 2013;86(3):343-58.

46. Thorpe KE, Zwarenstein M, Oxman AD, Treweek S, Furberg CD, Altman DG et al. A pragmatic-explanatory continuum indicator summary (PRECIS): a tool to help trial designers. J Clin Epidemiol. 2009;62(5):464-75. 

CHAPTER 7

\section{LESSONS LEARNED FROM CONDUCTING A}

PRAGMATIC, RANDOMIZED, CROSSOVER TRIAL ON ROBOT-ASSISTED GAIT TRAINING IN CHILDREN WITH CEREBRAL PALSY (PELOGAIT)

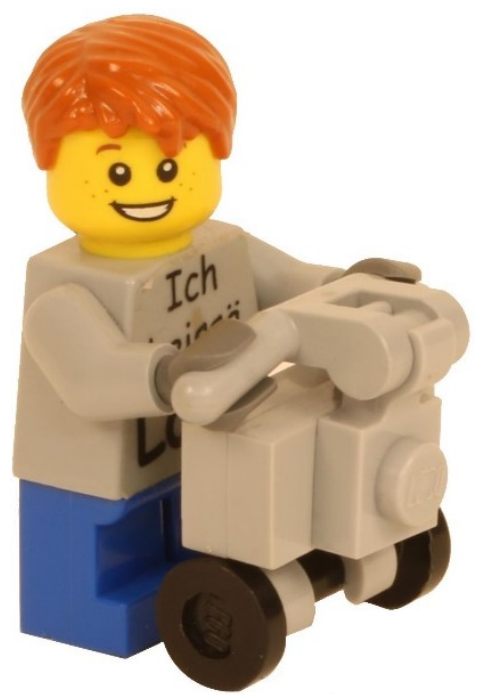

This chapter is in preparation for submission as:

Ammann-Reiffer C, Bastiaenen CHG, Meyer-Heim AD, van Hedel HJA. Lessons learned from conducting a pragmatic, randomized, crossover trial on robot-assisted gait training in children with cerebral palsy (PeLoGAIT). 


\section{ABSTRACT}

PURPOSE: To investigate the effectiveness of outpatient robot-assisted gait training (RAGT) in ambulatory children with spastic cerebral palsy (CP) and to highlight lessons learned from this trial.

METHODS: Children (11.3 years; 6.0-15.3years) were randomized to two different intervention sequences within a pragmatic crossover design. They performed 5 weeks of RAGT ( 3 sessions per week) and 5 weeks of usual care (UC). Dimension $\mathrm{E}$ of the Gross Motor Function Measure-88 (GMFM E) as primary outcome as well as GMFM D and timed walking tests were assessed before and after each treatment sequence, and after a 5-week follow-up.

RESULTS: GMFM E median change scores in the included 16 children (13 males) were -0.7 (interquartile range -2.8 to 3.5 ) after RAGT and 0 (-2.4 to 2.4) after UC. Neither GMFM E nor any secondary outcome measure changed significantly after RAGT or UC, nor were any period, follow-up, or carry-over effects observable.

CONCLUSIONS: The families' positive feedbacks were not reflected by our outcome measures. Children's personalized rehabilitation goals should therefore carefully be monitored with individualized measurement instruments. Furthermore, RAGT should be embedded as an add-on therapy in a holistic treatment approach, as it cannot cover all aspects relevant to gait.

TRIAL REGISTRATION NUMBER: NCT00887848. 


\section{INTRODUCTION}

Cerebral palsy (CP) is one of the most common physical disabilities, which affects about 2-3 children per 1'000 live births in the Western countries. ${ }^{1}$ It affects multiple dimensions of health, and children with $\mathrm{CP}$ are often restricted regarding independent mobility and daily walking activity, with one in three not being able to walk. ${ }^{2}$ Thus, improving gait function in these children is often one of the main goals of the families and the rehabilitation team. ${ }^{3,4}$ Pediatric neurorehabilitation addresses this goal with various impairment-based (i.e., focusing on the body structures and functions of the International Classification of Functioning, Disability and Health: Children and Youth Version (ICF-CY)) or more and more task-specific approaches (i.e., focusing on the activity component of the ICF-CY). ${ }^{5} \mathrm{~A}$ meta-analysis showed a substantial effect of different task-specific gait training interventions (including conventional overground training, treadmill training with and without body weight support, robot-assisted gait training (RAGT), and gait training on an electromechanical gait trainer) on walking speed in children with CP. ${ }^{5}$ Additionally, Zwicker and Mayson ${ }^{6}$ found positive results when summarizing the evidence of several systematic reviews on different kinds of treadmill training in children with neuromotor disorders.

New robotic rehabilitation technologies have revolutionized the neurorehabilitation field during the last 15 years and have increasingly been implemented in the clinical setting. ${ }^{7}$ They facilitate longer training sessions with more repetitions in a consistent movement pattern and provide an attractive and entertaining training opportunity due to the implementation of serious games. Further, the training parameters are automatically recorded and allow for a better comparison of training intensity across different centers. One of these rehabilitation robots is the driven gait orthosis Lokomat ${ }^{\circledR}$ (Hocoma AG, Volketswil, Switzerland), which enables a robot-assisted gait training (RAGT) for children starting at an early age of approximately five years. ${ }^{8}$ The Lokomat automates gait therapy on a treadmill by two actuated leg orthoses, which are available in pediatric and adult sizes. ${ }^{8,9}$ These orthoses are individually adapted to the patient's legs and are attached with cuffs of various available sizes, while the patient is secured using a counter system with a harness providing partial 
body-weight support. The Lokomat offers many possibilities for variations of training parameters, such as range of motion, gait speed, amount of body-weight support, and guidance force. ${ }^{10}$ Such modern technology raises high expectations in parents of children with CP regarding gains in their child's walking abilities and the development of a physiological gait pattern. ${ }^{3}$ For the therapy team, it is often a challenge to meet these expectations appropriately; the more so as scientific evidence in this area shows large deficits. Although studies have provided evidence on the Lokomat's feasibility and safety in pediatric populations, 8,11 information on the optimal frequency and duration of such interventions as well as the intensity and dosage of the training parameters is sparse. A recent systematic review on the optimal application mode and the effectiveness of RAGT in children with various gait disorders concluded that no clear delivery modes for RAGT in children exist and that evidence of its effectiveness is still weak and inconsistent. ${ }^{12} \mathrm{~A}$ main reason for the weak evidence is that most published results were derived from uncontrolled single case studies or case series ${ }^{13-18}$ and only a few randomized controlled trials (RCT) have been published. ${ }^{19-23}$ All these RCTs were performed with an immense effort regarding intervention and outcome measurements. Unfortunately, most of them prompted questions regarding their internal validity with limitations concerning randomization procedure, ${ }^{19,20}$ inconsistencies between inclusion criteria and included patient sample, ${ }^{19}$ a large dropout rate, ${ }^{20}$ and serious statistical flaws..$^{21,22}$ Additionally, two studies seemed to be based on the same patient sample without further commenting on it. ${ }^{21,22}$

When we started our RCT in 2009, only the results of the first uncontrolled single case studies and case series studies were available. As we were interested in the effects of RAGT as it is performed in our rehabilitation center, we applied a pragmatic trial design. ${ }^{24}$ This study design allows evaluating the effects of an intervention under the usual everyday conditions in which it is applied. ${ }^{25}$ We further chose to apply a crossover design, which enables each child to receive both treatments in a random order. As each patient can act as his or her own control, and variation within a person is usually less than between patients, the treatment effect can be estimated more precisely. With that, it requires only about half the number of patients needed in a 
parallel group design. ${ }^{26}$ As pediatric neurorehabilitation is generally faced with low case numbers, a reduction in sample size needed is an essential factor in this research field. We, therefore, accepted possible disadvantages of the crossover design like carry-over or period effects and longer study duration for the participants and addressed these statistically.

Thus, this research project aimed to investigate the effectiveness of RAGT on improvements of functional gait parameters in ambulatory children with cerebral palsy in a pragmatic, randomized, crossover trial. We hypothesized that a 5-week period of RAGT on the Lokomat in an outpatient setting would be superior concerning the improvement in gait function when compared to a 5-week phase of standard treatment in children with a bilateral spastic CP within a crossover design with a randomized treatment sequence. For this, we measured the dimension $\mathrm{E}$ of the Gross Motor Function Measure-88 (GMFM-88) as the primary outcome measure and collected several baseline variables and secondary outcome measures, mainly on the activity component of the ICF-CY.

After a study duration of more than eight years (compared to planned four years), we decided to stop the trial mainly due to severe recruitment difficulties, which we could not overcome despite several measures that were taken in the course of the trial. In this article, we report on the results of the 16 patients we have included until study termination, and address the lessons learned from our study, on the one hand from the content side regarding the Lokomat intervention, and on the other hand regarding methodological aspects.

\section{MATERIALS AND METHODS}

The study was designed in accordance with the Declaration of Helsinki ${ }^{27}$ and the extensions of the CONSORT (Consolidated Standards of Reporting Trials) statement for non-pharmacologic treatment interventions ${ }^{28}$ and pragmatic trials. ${ }^{29}$ It was approved by the Ethics Committee Zurich and registered on ClinicalTrials.gov (NCT00887848) on 23 April 2009. Written informed consent and assent was obtained from all the legal guardians and the child by the study coordinator (C.A.) prior to participation. 


\section{Design and setting}

The methods of this study are outlined in detail in the published study protocol. ${ }^{24}$ In short, the study was designed as a bicenter, pragmatic, randomized, crossover trial. It was carried out in the outpatient setting of the rehabilitation center of the University Children's Hospital Zurich in Affoltern am Albis and the Pediatric Therapy Center of the Reha Rheinfelden (Switzerland).

\section{Participants}

Children aged 6 to 18 years with a bilateral spastic CP and a Gross Motor Function Classification System (GMFCS) level II-IV who were able to walk at least 14 meters with or without walking aids were eligible for study participation. They had to be able to follow instructions and communicate pain or discomfort. Exclusion criteria were neuro- or orthopedic surgery on the lower extremity or trunk within the last six months, having participated in another Lokomat training regime within the previous six months as well as a change in concomitant drug therapy within the last four weeks before or during the study period. Further, children showing contraindications as outlined in the Lokomat manufacturer's manual, for example, severe contractures (i.e., $>20^{\circ}$ knee extension deficit, $>40^{\circ}$ hip extension deficit), bone fractures, open skin lesions or circulatory problems, were excluded.

Recruitment happened through announcements outside the rehabilitation center in schools for children with special needs, in- and outpatient pediatric clinics, and physical therapy practices. Parents of children with previous RAGT experience were informed by a letter of the center's head physician and information on the study was published online.

\section{Randomization}

Children were randomized to two different pre-specified sequences of interventions. The two interventions were RAGT as treatment $(T)$ on the Lokomat in the outpatient setting of the RCA and usual care as control $(C)$ in the home environment of the child. A child could be randomized to a T/C sequence (TC-group) or a C/T/C sequence (CTC-group). Randomization into the two groups was performed using a minimization method with a random factor of 0.9 . We included the factors severity of impairment (GMFCS-level II or III/IV), age (6-11 years or 11-18 years) and 
Botulinum Toxin A-treatment (present or absent) in the preceding 6 months, as we assumed that these factors could influence the effect of the intervention. To ensure concealed allocation, the randomization list, which was generated on www.randomizer.at by a person not belonging to the study team, was stored off-site. As soon as the study coordinator had received written informed consent, information about the included child's group allocation was requested via phone or e-mail.

\section{Intervention}

$\operatorname{RAGT}(T)$

RAGT on the Lokomat was applied three times per week over five consecutive weeks with a maximum of 45 minutes of effective walking time per session. Trained and experienced therapists, who were used to working with children, performed the training sessions, which were executed according to recently published recommendations. ${ }^{10}$ Serious games and other available motivating strategies were used to increase the children's adherence to the training. Body-weight support, gait speed, training duration, as well as guidance force of the Lokomat were individually adjusted according to the child's abilities and modified within training sessions and during the course over time. Standard therapies could be continued during the RAGT period, but should not include specific walking training. Type and dosage of concomitant treatment were recorded in a logbook.

\section{Usual care (C)}

Usual care normally consisted of one to two individual sessions of physiotherapy per week. Additionally, hippotherapy, circuit training, or occupational therapy could be elements of standard treatment. These therapies, which were individually customized to the needs of the child, were often implemented in the school setting in the child's home environment. Usual care treatment did not specifically address gait training but rather consisted of elements addressing range of motion, muscle tone reduction, balance, activities of daily living, etc. Responsibility, planning, and content of the C-treatment were entirely in the hand of the child's therapist in the home environment. Information about the type, duration, and frequency of all therapies was documented in a logbook by the parents. 
The duration of study participation and number of assessment time points varied dependent on group allocation between 11 weeks with three assessment time points (TC-group) and 16 weeks with four assessment time points (CTC-group) (see Figure 7.1.).

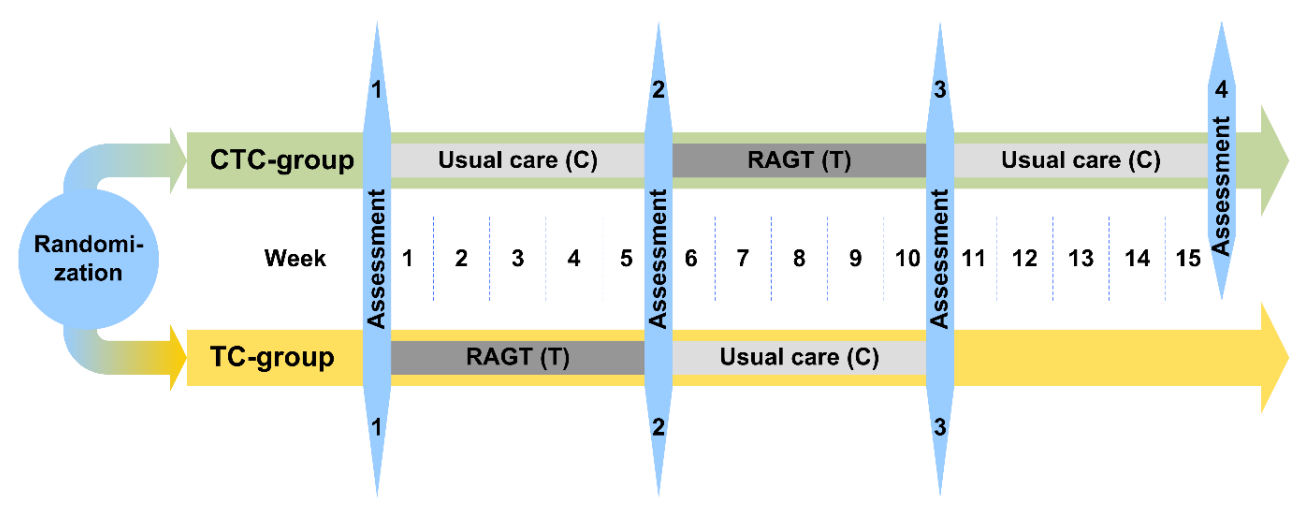

Figure 7.1. Overview of the outcome measures and the measurement time points per group.

Children randomized to the CTC-group with the intervention sequence usual care/robot-assisted gait training (RAGT)/usual care were measured at four different time points within 16 weeks. Children randomized to the TC-group underwent the intervention sequence RAGT/usual care and were measured at three different time points within 11 weeks. Differences regarding intervention time and numbers of measurement time points were deliberately chosen to reduce the burden on children and families as much as possible.

\section{Outcome measures}

Primary outcome measure

The percentage score of the dimension E of the GMFM-88 (GMFM E) was used as primary outcome measure. ${ }^{30}$ It assesses the gross motor function with regard to walking ability and consists of 24 items, which are scored on a 4-point ordinal scale. The test was performed without walking aids, regardless of the children's GMFCSlevel. A GMFM-certified therapist instructed and rated the child. While the psychometric properties of the total GMFM-88 are well explored in children with CP, there is limited positive evidence for the responsiveness of dimension E. ${ }^{31}$

\section{Secondary outcome measures}

We used the percentage score of the dimension D of the GMFM-88 (GMFM D) to assess the gross motor function with regard to standing ability. ${ }^{30}$ The GMFM D consists of 13 items, which were tested and scored in the same way as described 
for GMFM E. There is limited positive evidence for its responsiveness, ${ }^{31}$ while reliability seems to be high. ${ }^{32}$

The 6-minute walk test (6MinWT) was performed on a 30-meter long corridor with poles at each end and standardized instructions and encouragement for the child. ${ }^{33}$ While there is moderate evidence for the test-retest reliability of the 6MinWT, responsiveness has not been established in children with $\mathrm{CP} .{ }^{31}$

We assessed gait speed with the 10-meter walk test at self-selected (10MWTss) and fast speed (10MWTfast). Children walked on a 14-meter long track with orthoses and walking aids usually used in daily life. Each test was performed twice and time needed for the middle 10 meters was measured with a stopwatch. Evidence for testretest reliability of the $10 \mathrm{MWTfast}$ is moderate in children with CP. ${ }^{31}$

Further, measures of body function (range of motion, modified Ashworth scale, and manual muscle tests of the lower extremities) were evaluated for descriptive purposes. ${ }^{34,35}$

\section{Sample size and power calculation}

For the sample size calculation, we used the PASS Power Analysis and Sample Size software program (NCSS, LLC, Kaysville, USA) with a Minimum Important Clinical Difference (MCID) of 3.7\%-points in the GMFM E, which was reported by Wang and Yang, ${ }^{36}$ and a standard deviation of 6.8\%-points, which was derived from our data. ${ }^{14}$ With a power of $80 \%$ and a significance level of $5 \%$, it indicated a sample size of 30 to be sufficient for a $2 \times 2$ crossover design. We increased this number to 34 to allow for a predicted dropout rate of about $10 \%$.

\section{Statistical analysis}

The statistical analyses were carried out with SPSS 24 (IBM Corporation, New York, USA) and datasets of all study participants were analyzed on an intention-to-treat basis. Descriptive statistics are presented for the clinical characteristics of the study participants and the quantification of the T- and C-intervention. Because of ordinalscaled data or non-normal data distribution for the primary and secondary outcome measures, treatment effects, period effects as well as follow-up effects of the two different treatment interventions were analyzed with Wilcoxon signed-rank tests for 
paired samples, with the treatment effect being our primary end-point. Wilcoxon rank sum tests for unpaired samples were used to determine whether carryover effects were present. ${ }^{26}$ Ninety-five percent confidence intervals are presented with a twotailed level of significance set at $p<0.025$ for treatment and follow-up effects, and $p$ $<0.05$ for period and carryover effects. Additionally, we calculated effect sizes based on the $z$ values of the nonparametric Wilcoxon rank sum tests as $r=Z \div \sqrt{N}$ and interpreted $r \geq 0.1$ as small, $r \geq 0.3$ as moderate, and $r \geq 0.5$ as large effect. ${ }^{37,38}$ An illustration of all applied analyses is given in Figure 7.2.

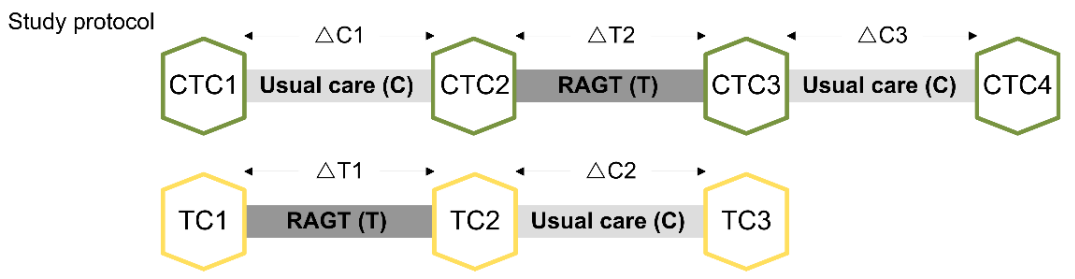

Analyses

\begin{tabular}{|c|c|c|}
\hline Treatment effects & ( $\triangle \mathrm{T} 1$ grouped with $\triangle \mathrm{T} 2)$ versus $(\triangle \mathrm{C} 1$ grouped with $\triangle \mathrm{C} 2) \Rightarrow$ & Paired analysis \\
\hline Period effects & ( $\triangle \mathrm{C} 1$ grouped with $\triangle \mathrm{T} 1$ ) versus $(\triangle \mathrm{T} 2$ grouped with $\triangle \mathrm{C} 2) \Rightarrow$ & Paired analysis \\
\hline Follow-up effects & ( $\mathrm{C}_{\mathrm{TC}} 3$ grouped with $\left.\mathrm{T}_{\mathrm{C}} 2\right)$ versus $\left(\mathrm{C}_{\mathrm{TC}} 4\right.$ grouped with $\mathrm{T}_{\mathrm{C}} 3$ ) & Paired analysis \\
\hline $\begin{array}{l}\text { Carry-over effects } \\
\text { (treatment by period } \\
\text { interaction) }\end{array}$ & $(\triangle \mathrm{C} 1+\triangle \mathrm{T} 2)$ versus $(\triangle \mathrm{T} 1+\triangle \mathrm{C} 2)$ & Unpaired analysis \\
\hline
\end{tabular}

Figure 7.2. Overview of the study protocol and the statistical analyses.

CTC1, baseline assessment in CTC-group; TC1, baseline assessment in TC-group; CTC2, intermediate assessment in CTC-group; TC2, intermediate assessment in TC-group; CTC3,: end assessment in CTCgroup; TC3, end assessment in TC-group; CTC4, follow-up assessment in CTC-group; $\triangle \mathrm{C} 1$, change during usual care in CTC-group; $\Delta \mathrm{C} 2$, change during usual care in TC-group; $\Delta \mathrm{C} 3$, change during followup in CTC-group; $\Delta \mathrm{T} 1$, change during robot-assisted gait training in TC-group; $\Delta \mathrm{T} 2$, change during robotassisted gait training in CTC-group.

\section{RESULTS}

The first child was enrolled into the study in October 2009. Due to severe recruitment difficulties and the already prolonged study duration, the study coordinator and the sponsor (H.v.H.) unanimously decided to close the trial untimely in March 2018. Until trial termination, 16 children and adolescents with a bilateral spastic CP had completed the study, without any dropouts or violation of their assigned treatment 
sequences. Descriptive characteristics of the study participants are presented in Table 7.1.

Table 7.1. Descriptive characteristics of the study participants $(n=16)$

\begin{tabular}{|c|c|c|c|c|}
\hline & & TC-group & СTC-group & Total \\
\hline \multirow{2}{*}{$\begin{array}{l}\text { Gender } \\
(\mathrm{n})\end{array}$} & male & 6 & 7 & 13 \\
\hline & female & 2 & 1 & 3 \\
\hline \multirow{3}{*}{$\begin{array}{l}\text { GMFCS-level } \\
\text { (n) }\end{array}$} & II & 5 & 4 & 9 \\
\hline & III & 2 & 3 & 5 \\
\hline & IV & 1 & 1 & 2 \\
\hline Age (years) & & $\begin{array}{r}11.4(6.0- \\
15.1)\end{array}$ & $\begin{array}{r}11.2(6.1- \\
15.3)\end{array}$ & $\begin{array}{r}11.3(6.0- \\
15.3)\end{array}$ \\
\hline Height $(\mathrm{cm})$ & & $146(118-178)$ & $142(110-174)$ & $144(110-178)$ \\
\hline Weight (kg) & & $41(24-67)$ & $43(18-81)$ & $42(18-81)$ \\
\hline \multirow{4}{*}{$\begin{array}{l}\text { Walking aids in } \\
\text { everyday life ( } n \text { ) }\end{array}$} & None & 5 & 4 & 9 \\
\hline & Walker & 2 & 2 & 4 \\
\hline & PA & 1 & 1 & 2 \\
\hline & Wheelchair & 0 & 1 & 1 \\
\hline \multirow{2}{*}{$\begin{array}{l}\text { Physiotherapy during } \\
\text { usual care period }\end{array}$} & Sessions/week & $2(1-3)$ & $2(1-3)$ & $2(1-3)$ \\
\hline & Minutes/week & $90(45-135)$ & $90(45-135)$ & $90(45-135)$ \\
\hline \multirow[t]{6}{*}{ Range of motion $\left({ }^{\circ}\right)$} & Hip flexion & & & $119(90-140)$ \\
\hline & Hip extension & & & $-2(-20-15)$ \\
\hline & Knee flexion (in & flexion) & & $145(110-165)$ \\
\hline & Knee extension & ip flexion) & & $-2(-20-15)$ \\
\hline & Ankle dorsiflexic & $\partial^{\circ}$ knee flexion) & & $5(-20-25)$ \\
\hline & Ankle plantarfle & & & $45(20-65)$ \\
\hline
\end{tabular}

The number of observations $(\mathrm{n})$ is presented for nominal and ordinal variables. Interval scaled variables are presented as mean and (range). TC-group, group with treatment-control sequence; CTC-group, group with control-treatment-control sequence; GMFCS, Gross Motor Function Classification System; PA, personal assistance.

Five of the children (TC-group: $n=3$; CTC-group: $n=2$ ) had previously undergone one or several surgical interventions at their lower extremities, with all of them being performed at least two years before study participation. Three children belonging to the TC-group had received Botulinum Toxin-A treatment (which was a patient factor 
within the minimization method) into muscles of the lower extremities within six months before their study participation.

Regarding the ICF-CY body function level, the median modified Ashworth score of all children was 0 with an interquartile range (IQR) of 1 . The children's muscle strength was moderate (median: 3; IQR: 1). Sagittal range of motion values of the lower extremities are listed in Table 7.1.

Treatment adherence of the participants was high. Except for one child, who performed 10 RAGT sessions, all others completed at least 14 of 15 sessions. Descriptive parameters of the RAGT sessions are listed in Table 7.2.

Table 7.2. Characteristics of the robot-assisted gait training (RAGT) dosage parameters (averaged over all training sessions per person)

\begin{tabular}{|c|c|c|c|}
\hline & Mean & Standard deviation & Minimum-Maximum \\
\hline RAGT session (n) & 14.3 & 1.2 & $10-15$ \\
\hline Gait speed $[\mathrm{m} / \mathrm{s}]$ & 1.4 & 0.1 & $0.5-2.3$ \\
\hline Body weight support [\%] & 27 & 0.8 & $0-78$ \\
\hline Guidance force [\%] & 94 & 4 & $55-100$ \\
\hline Session duration [min:sec] & $39: 45$ & $4: 50$ & $15: 52-47: 00$ \\
\hline Distance walked per session [m] & 935 & 159 & $129-1$ '636 \\
\hline Distance walked in total $[\mathrm{m}]$ & $13^{\prime} 519$ & 3'641 & 2'508-19'843 \\
\hline
\end{tabular}

Additionally to the RAGT sessions, children attended during the T-sequence on average $125 \mathrm{~min} \pm 148 \mathrm{~min}(0-540 \mathrm{~min})$ of physiotherapy and four children also continued with their hippotherapy sessions ( $41 \mathrm{~min} \pm 85 \mathrm{~min}$; 0-300 min). During the Cintervention, physiotherapy added up to $282 \mathrm{~min} \pm 256 \mathrm{~min}\left(0-1{ }^{\prime} 020 \mathrm{~min}\right)$ and seven children attended hippotherapy (63min $\pm 82 \mathrm{~min} ; 0-225 \mathrm{~min})$.

\section{Primary outcome measure}

Results regarding the primary outcome are described in Table 7.3. GMFM E percentage scores did not increase significantly after the RAGT or after the usual care period. Neither a treatment effect nor a period, follow-up, or carry-over effect was observable ( $p=0.91 / 0.61 / 0.75 / 0.40$, respectively). The effect size did not indicate any effect either. Figure 7.3. illustrates children's individual GMFM E change scores after RAGT and usual care. 
Table 7.3. Scores and change scores at different time points, treatment effects and effect sizes of primary and secondary outcome measures

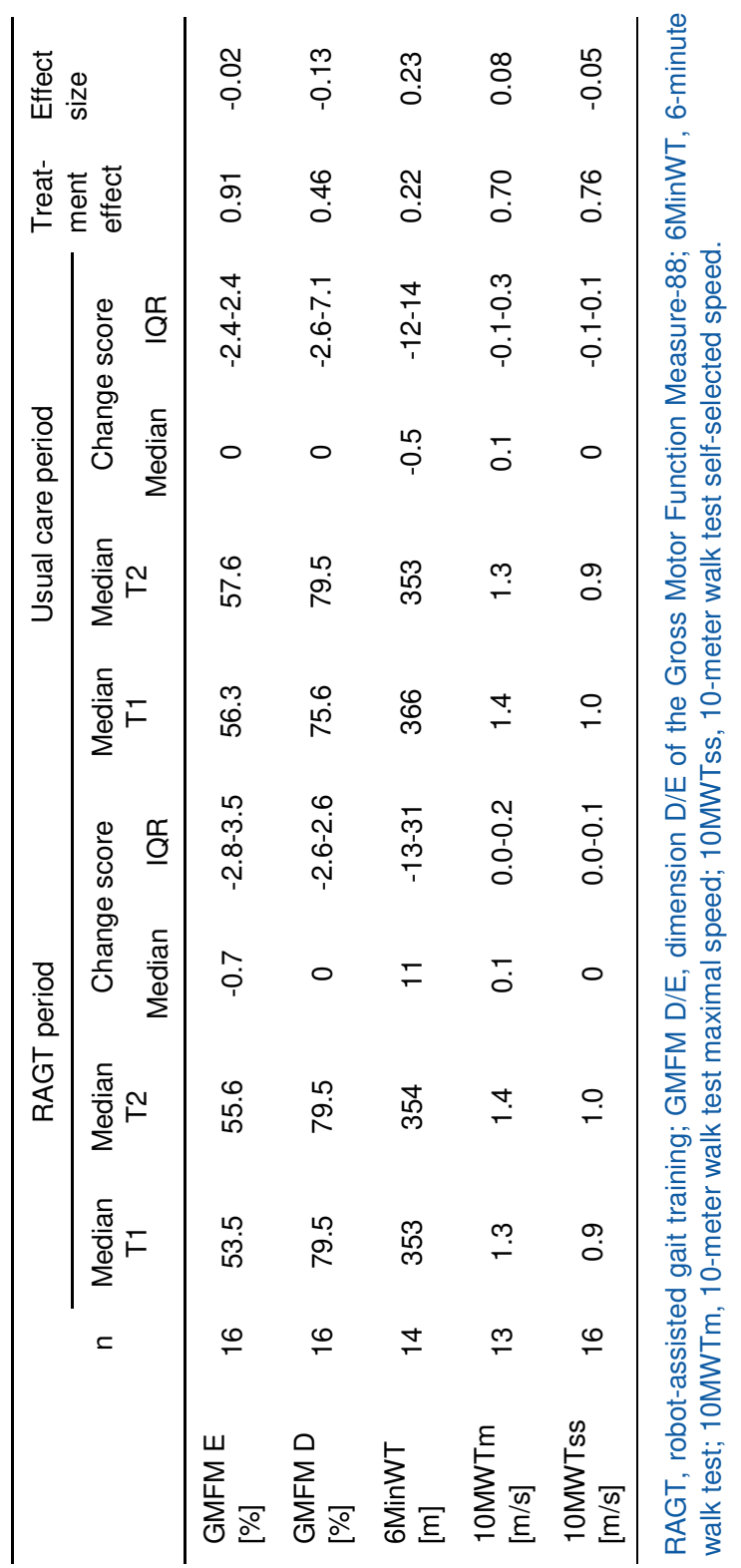




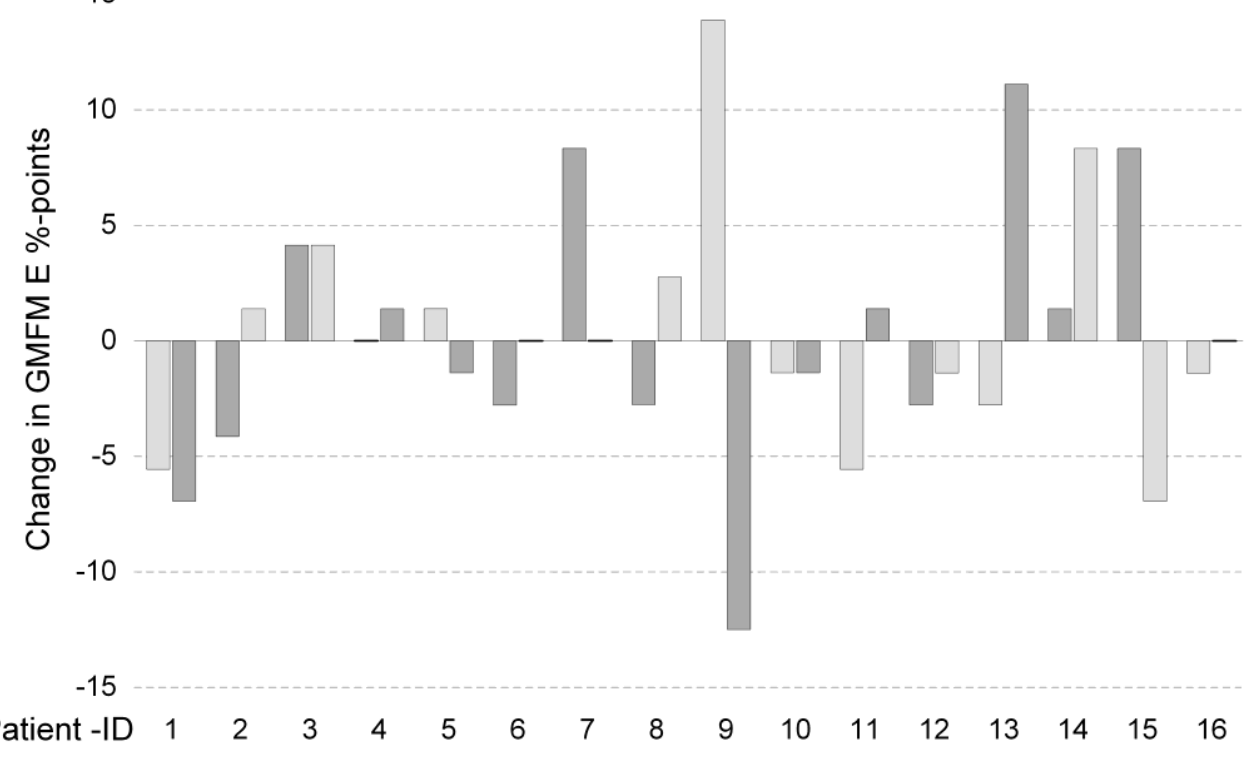

Figure 7.3. Individual effects of robot-assisted gait training (RAGT) and usual care on Gross Motor Function Measure dimension E (GMFM E) change scores. The order of the bars reflects the chronological sequence of the treatment.

usual care

robot-assisted gait training

\section{Secondary outcome measures}

Results of the secondary outcomes are described in Table 7.3. None of the secondary outcome measures indicated a significant treatment effect. Effect sizes did not indicate any effect except for the 6MinWT, which showed a small effect. No period, follow-up, or carry-over effects were observed in any of the secondary outcome measures.

\section{DISCUSSION}

We investigated the effectiveness of RAGT on improving walking abilities in ambulatory children with bilateral spastic CP. For this, we used a pragmatic crossover design with randomized treatment sequences. We stopped the trial early, i.e., after more than eight years, when only 16 of the intended 30 patients (34 including dropouts) had finished the protocol, due to recruitment difficulties. Despite having taken various measures to increase the number of participating children (prolonged trial duration, broadened eligibility criteria (extended age limit from 16 to 
18 years, inclusion of children with GMFCS level IV, if they were able to walk 14 meters), expansion from a mono- to a bi-center trial), we had to recognize that achieving the calculated sample size within the next one to three years would have been unattainable. Unfortunately, discontinuation and nonpublication of RCTs conducted in children have a high prevalence, with difficulties in patient recruitment being the most commonly mentioned reason. ${ }^{39}$ Nevertheless, it is crucial to publish also results of such a trial for several reasons: Although we stopped the study untimely because the inclusion rate was distinctly lower than anticipated over the years, our results provide some indications regarding the effectiveness of RAGT in children with CP. Additionally, they give valuable information for the design and realization of a study concerning children with CP. ${ }^{40}$

Hopefully, other clinicians or researchers can capitalize on the problems we were faced with when running this study.

We had hypothesized that a 5-week phase of RAGT would be superior regarding the improvement of walking abilities when compared to standard treatment with conventional physiotherapy for the same duration. The included participants covered a broad spectrum of our defined inclusion criteria regarding age, GMFCS-levels, and walking abilities. After about half of the intended number of patients - who covered a broad spectrum of our defined inclusion criteria regarding age, GMFCS-levels, and walking abilities - having finished the protocol, we could not confirm this hypothesis. While the lower power may have hampered statistically significant changes, the effect sizes of the primary, as well as the secondary outcome measures, were not promising either.

Existing evidence on the effectiveness of RAGT is weak and inconsistent. ${ }^{12}$ Only four RCTs on RAGT with the Lokomat in children with CP have been published. Druzbicki et al. found no effects on kinematic and temporospatial gait parameters after four weeks with 20 sessions of RAGT in children with GMFCS levels II and III. ${ }^{20}$ Peri et al. compared different protocols of gait training interventions with RAGT and task-oriented physiotherapy in 44 children with a GMFCS level I to III. ${ }^{23}$ While spatiotemporal and kinematic gait parameters did not change either in any of the protocols, GMFM E scores improved significantly after 40 RAGT sessions over a 
duration of 10 weeks, as well as after 40 physiotherapy sessions with standardized gait targeting exercises for the same period. Improvements (RAGT $+3.5 \%$, gait exercises $+3.2 \%$ ) exceeded the MICD of a large effect size in both groups. The GMFM D also improved considerably in both groups (RAGT $+3 \%$, gait exercises $+5 \%$ ), while the 6 MinWT only increased in the RAGT group $(+21 \mathrm{~m})$. However, these results did not reach statistical significance, and no differences between the intervention protocols were present. Surprisingly, the protocols with mixed interventions (a combination of 20 RAGT and 20 conventional gait therapy sessions over 4 or 10 weeks) did not reveal any significant changes at all. In contrast to the studies described above, the two RCTs conducted by Wallard et al. (both including the identic patient sample of 30 children with spastic CP, GMFCS-level II) found significant improvements in diverse kinematic parameters on the lower and upper extremities as well as in GMFM D and E scores after 20 sessions of RAGT. ${ }^{21,22}$ However, these two studies prompt various questions regarding the randomization and concealment of allocation sequence procedure, the statistical analyses, and the standardization of the therapeutic interventions. Further, the clinical relevance of significant improvements in kinematic parameters of only a few degrees is at least questionable. The lower-level design studies on RAGT in children, which did not include a control group, yielded inconsistent results regarding improvement in gait speed, gait endurance, and gross motor function. ${ }^{12}$

The results of our study imply that RAGT is not effective in this patient population. Besides the statistically insignificant results, the effect sizes, except those of the $6 \mathrm{MinWT}$, did not indicate any positive results either. Taking our findings and experiences conducting the trial over the past years into consideration, we have several points to address. Some aspects refer to a conceptual level regarding developing insights into the contents of a rehabilitation process based on the approach of the ICF-CY, and others to gaining more profound methodological insights into working with a pragmatic trial design in general as well as in this particular patient group.

Half of the children in our study had previous RAGT experience and completed their second or third RAGT period. Schroeder et al. found that repetition of RAGT 
negatively correlates with improvement in GMFM scores, ${ }^{41}$ although we could not find such a relation in our data. Furthermore, evidence exists that development of gross motor function in children with CP may vary in countries with different health services systems. ${ }^{42,43}$ In Switzerland, as in many other western European countries and Canada, children with $\mathrm{CP}$ are embedded in a timely and constant rehabilitation regime. Thus, children in our study already received a relatively large amount of therapy before their study participation to promote their motor abilities as much as possible. This observation leads us to the first valuable lesson learned from this research: comparing single therapy interventions like RAGT against other therapeutic approaches is - although this was standard practice when we started our study - of questionable relevance for the rehabilitation field nowadays, where different disciplines and therapies are mostly combined to a complete package. Single aspects of the treatment package (such as RAGT) should only be investigated as an add-on option within the whole treatment approach and not be applied as stand-alone therapies. Otherwise, such studies could evoke problems regarding the contrast between the different study arms. This was probably also the case in our study, as most of the children were enrolled in a regular therapy program in their own environment, which they accomplished during the usual care period, and more than half of the children at least partially also during the RAGT period.

Concerning the RAGT intervention itself, we tried to follow a pragmatic approach as much as possible. As methods and principles of RAGT application and the accompanying software changed over the last eight years, we always aimed at applying the current state-of-the-art training strategies when performing RAGT. For example, we continually implemented the latest Lokomat software and serious games and adjusted the training parameters individually to promote active participation in each child. In regular practice, we do not opt for an isolated Lokomat training in our center but always combine it with physiotherapy and sports therapy lessons, focusing on the same individualized goals within a multidisciplinary rehabilitation approach. These goals are defined in advance together with the children and parents to cover the areas that are most relevant to each family and thus mostly concern the activity and participation component of the ICF-CY. For the 
trial, we selected the GMFM E as our primary outcome, because the GMFM was considered the gold standard for the evaluation of gross motor function in children with CP. This leads to a next lesson learned from our study: A pragmatic study approach should reflect the personalized rehabilitation goals as best as possible. Thus, care should be taken that an individualized measurement instrument is used as a primary outcome. Such measures will ensure that intervention studies examine results relevant to both the child and family as well as the rehabilitation team. For this purpose, for example, the Goal Attainment Scale may be a promising option, which quantifies the achievement of goals in a standardized way, while these goals are set for each child individually. ${ }^{44,45}$

Our training protocol prescribed three RAGT sessions per week over a period of five weeks - a frequency and duration that we had considered clinically adequate and feasible on an outpatient basis. A 4-5-week period of 15-20 RAGT sessions also conforms with what is usually financed in children with $\mathrm{CP}$ by the health insurances in Switzerland. However, Peri et al. had adopted 40 therapy sessions within ten weeks in their groups with significant and clinically meaningful improvements, while their group with a mixed protocol of 20 RAGT and 20 physiotherapy sessions over four weeks did not improve. ${ }^{23}$ The four-week RAGT intervention of Druzbicki et al. did not result in a positive effect either. ${ }^{20}$ Apparently, the duration of the applied RAGT period could be a crucial factor for the potential of improvement in these children.

A crucial methodological lesson learned from this trial involves the recruitment of study children. Due to our intention to explore the effectiveness of RAGT as a single intervention compared to a usual care approach, we decided not to conduct this study in our regular inpatient setting. In our rehabilitation center, allocated children stay in the inpatient setting for many weeks and visit various therapy sessions and school in our center, as our center is the only rehabilitation center particularly for children in Switzerland. Only offering RAGT over several weeks instead of a whole rehabilitation treatment package would not have been acceptable under these conditions, neither for the families nor the treatment team. This decision led us to take a more explanatory approach concerning the aspect of patient recruitment. 
Despite the fact that we also treat children on an outpatient basis in our center, this fact, in retrospect, turned out to be one of the reasons that we only were able to recruit half of the anticipated children. Although we had taken steps to keep the families' effort of study participation within reasonable limits, it became apparent that most families preferred to arrange the RAGT period during their child's school holidays, to limit the burden acting on the child as much as possible. The more so since the geographically decentralized location of our center brought considerable travel expenses for many families. These issues reduced the number of participants from the anticipated ten children to maximally two to three per year. We expanded the mono- to a bi-center trial after five years, enlisting the support of the Pediatric Therapy Center of the Rehabilitation center in Rheinfelden, the only other center with a pediatric Lokomat in Switzerland. Unfortunately, despite the local study coordinator's high motivation and effort, no single child could be recruited in this second center.

To avoid such problems, recruitment should focus as much as possible on the patient population represented in everyday clinical practice. In this way, logistical challenges, planning efforts, and resources of participating and executing parties can be kept within a better manageable and foreseeable range. Moreover, the research question can be answered within the context the question originated and not in a more artificial situation.

In general, it should be considered that such design choices towards a more pragmatic or explanatory study design may affect validity, generalizability, precision, and feasibility. ${ }^{46}$ Nowadays, the PRECIS-2 tool, which was developed to support researchers in designing trials, would be a helpful instrument to make informed decisions about the trials' position on the pragmatic-explanatory continuum. ${ }^{47}$

We opted for a crossover design because it was regarded the optimal study design for small patient groups when the study protocol was established. Recognizing the lessons mentioned above as well as the present disadvantages of this design (longer trial duration, potential carryover and period effects, higher drop-out rates, sophisticated statistical analyses), ${ }^{48}$ other study designs, as n-of- 1 trials, single case 
methodology designs or observational designs, could be considered as possible alternatives to the chosen crossover design.

\section{CONCLUSION}

The results of our study indicate that 15 sessions of RAGT within a 5 -week period are not effective in improving walking abilities in ambulatory children with CP. With that, it adds a further piece to the very limited evidence of RAGT in children with CP. We applied RAGT as a single intervention compared to usual care, in line with the methodological point of view when designing the study. Our results must, therefore, also be interpreted in this light. Nowadays, clinical recommendations are to embed its application in a holistic treatment with other therapies. ${ }^{10}$

An inpatient approach, offering the possibility of higher RAGT dosages as an addon therapy element within an individualized holistic treatment package - as it is common practice in our center - may be a more promising and pragmatic, although still challenging, strategy to investigate the effect on walking abilities in children with $\mathrm{CP}$ living in Switzerland. Further high-level design studies are on the way, ${ }^{49}$ and until the publication of their results, we have to monitor each child carefully during a RAGT intervention to determine whether benefits are present that justify the efforts and costs of this highly specialized treatment approach.

\section{REFERENCES}

1. Rosenbaum $P$, Paneth $N$, Leviton $A$, et al. A report: The definition and classification of cerebral palsy April 2006. Dev Med Child Neurol. 2007;49(SUPPL.109):8-14.

2. Novak I, Hines M, Goldsmith S, Barclay R. Clinical prognostic messages from a systematic review on cerebral palsy. Pediatrics. 2012;130(5):e1285-312.

3. Beveridge B, Feltracco D, Struyf J, et al. "You gotta try it all": Parents' Experiences with Robotic Gait Training for their Children with Cerebral Palsy. Phys Occup Ther Pediatr. 2015;35(4):327-341.

4. Vargus-Adams JN, Martin LK. Domains of importance for parents, medical professionals and youth with cerebral palsy considering treatment outcomes. Child Care Health Dev. 2011;37(2):276-281.

5. Moreau NG, Winter AB, Bjornson K, Hobbs A, Soileau M, Lahasky K. Effectiveness of Rehabilitation Interventions to Improve Gait Speed in Children With 
Cerebral Palsy: Systematic Review and Meta-analysis. Phys Ther. 2016;96(12):1938-1954.

6. Zwicker JG, Mayson TA. Effectiveness of Treadmill Training in Children With Motor Impairments : An Overview. Pediatr Phys Ther. 2010;22:361-377.

7. Tefertiller C, Pharo B, Evans N, Winchester P. Efficacy of rehabilitation robotics for walking training in neurological disorders: A review. J Rehabil Res Dev. 2011;48(4):387-416.

8. Meyer-Heim A, Borggraefe I, Ammann-Reiffer C, et al. Feasibility of roboticassisted locomotor training in children with central gait impairment. Dev Med Child Neurol. 2007;49(12):900-906.

9. Colombo G, Joerg M, Schreier R, Dietz V. Treadmill training of paraplegic patients using a robotic orthosis. J Rehabil Res Dev. 2000;37(6):693-700.

10. Aurich T, Warken B, Graser J V., et al. Practical Recommendations for RobotAssisted Treadmill Therapy (Lokomat) in Children with Cerebral Palsy: Indications, Goal Setting, and Clinical Implementation within the WHO-ICF Framework. Neuropediatrics. 2015;46(4):248-260.

11. Borggraefe I, Klaiber M, Schuler $\mathrm{T}$, et al. Safety of robotic-assisted treadmill therapy in children and adolescents with gait impairment: a bi-centre survey. Dev Neurorehabil. 2010;13(2):114-119.

12. Lefmann S, Russo R, Hillier S. The effectiveness of robotic-assisted gait training for paediatric gait disorders: systematic review. J Neuroeng Rehabil. 2017;14(1):1.

13. Meyer-Heim A, Borggraefe I, Ammann-Reiffer C, et al. Feasibility of roboticassisted locomotor training in children with central gait impairment.pdf. Dev Med Child Neurol. 2007;49:900-906.

14. Meyer-Heim A, Ammann-Reiffer C, Schmartz A, et al. Improvement of walking abilities after robotic-assisted locomotion training in children with CP. Arch Dis Child. 2009;94(8):615-620.

15. Borggraefe I, Meyer-Heim A, Kumar A, Schaefer J, Berweck S, Heinen F. Improved gait parameters after robotic-assisted locomotor treadmill therapy in a 6year-old child with cerebral palsy. Mov Disord. 2008;23(2):280-283.

16. Borggraefe I, Schaefer JS, Klaiber M, et al. Robotic-assisted treadmill therapy improves walking and standing performance in children and adolescents with cerebral palsy. Eur J Paediatr Neurol. 2010;14(6):496-502.

17. Borggraefe I, Kiwull L, Schaefer J, et al. Sustainability of motor performance after robotic-assisted treadmill therapy in children: an open, non-randomized baselinetreatment study. Eur J Phys Rehabil Med. 2010;46(2):125-131.

18. Schroeder A, Homburg M, Warken B, et al. Prospective controlled cohort study to evaluate changes of function, activity and participation in patients with bilateral 
spastic cerebral palsy after Robot-enhanced repetitive treadmill therapy. Eur $J$ Paediatr Neurol. 2014;18(4):502-510.

19. Smania N, Bonetti $P$, Gandolfi $M$, et al. Improved gait after repetitive locomotor training in children with cerebral palsy. Am J Phys Med Rehabil. 2011;90(2):137149.

20. Druzbicki M, Rusek W, Snela $S$, et al. Functional effects of robotic-assisted locomotor treadmill therapy in children with cerebral palsy. J Rehabil Med. 2013;45(4):358-363.

21. Wallard L, Dietrich G, Kerlirzin Y, Bredin J. Robotic-assisted gait training improves walking abilities in diplegic children with cerebral palsy. Eur J Paediatr Neurol. 2017;21(3):557-564.

22. Wallard L, Dietrich G, Kerlirzin Y, Bredin J. Effect of robotic-assisted gait rehabilitation on dynamic equilibrium control in the gait of children with cerebral palsy. Gait Posture. 2017;11(60):55-60.

23. Peri E, Turconi A, Biffi E, et al. Effects of dose and duration of Robot-Assisted Gait Training on walking ability of children affected by cerebral palsy. Technol Heal Care. 2017;9(4):671-681.

24. Ammann-Reiffer C, Bastiaenen CHG, Meyer-Heim AD, van Hedel HJA. Effectiveness of robot-assisted gait training in children with cerebral palsy: A bicenter, pragmatic, randomized, cross-over trial (PeLoGAIT). BMC Pediatr. $2017 ; 17(1)$.

25. Thorpe $\mathrm{K}$, Zwarenstein $\mathrm{M}$, Oxman A, et al. A pragmatic-explanatory continuum indicator summary (PRECIS): a tool to help trial designers. J Clin Epidemiol. 2009;62(5):464-475.

26. Senn S. Cross-over Trials in Clinical Research. 2nd ed. Chichester: John Wiley \& Sons Ltd.; 2002.

27. World Medical Association Declaration of Helsinki. Ethical principles for medical research involving human subjects. JAMA. 2013;310(20):2191-2194.

28. Boutron I, Moher D, Altman D, Schulz K, Ravaud P. Extending the CONSORT statement to randomized trials of nonpharmacologic treatment: explanation and elaboration. Ann Intern Med. 2008;148(4):295-309.

29. Zwarenstein M, Treweek S, Gagnier J, et al. Improving the reporting of pragmatic trials: an extension of the CONSORT statement. BMJ. 2008;337(a2390).

30. Russell DJ, Rosenbaum PL, Cadman DT, Gowland C, Hardy S, Jarvis S. The gross motor function measure: a means to evaluate the effects of physical therapy. Dev Med Child Neurol. 1989;31:341-352. 
31. Ammann-Reiffer C, Bastiaenen CHG, de Bie RA, van Hedel HJA. Measurement Properties of Gait-Related Outcomes in Youth With Neuromuscular Diagnosis: A systematic review. Phys Ther. 2014;94(8):1067-1082.

32. Bjornson K, Graubert C, McLaughlin J. Test-retest reliability of the gross motor function measure in children with cerebral palsy. Pediatr Phys Ther. 2000;12(4):200202.

33. American Thoracic Society. ATS Statement: Guidelines for the Six-Minute Walk Test. Am J Respir Crit Care Med. 2002;166:111-117.

34. Fosang AL, Galea MP, McCoy AT, Reddihough DS, Story I. Measures of muscle and joint performance in the lower limb of children with cerebral palsy. Dev Med Child Neurol. 2003;45(10):664-670.

35. Hislop H, Montgomery J. Daniels' and Worthinghams Muskeltests - Manuelle Untersuchungstechniken. 7th ed. München: Urban und Fischer Verlag; 1999.

36. Wang $\mathrm{H}-\mathrm{Y}$, Yang $\mathrm{YH}$. Evaluating the responsiveness of 2 versions of the gross motor function measure for children with cerebral palsy. Arch Phys Med Rehabil. 2006;87(1):51-56.

37. Cohen J. Statistical Power Analysis for the Behavioral Sciences. 2. Hillsdale: Lawrence Erlbaum Associates; 1988.

38. Fritz CO, Morris PE, Richler JJ. Effect size estimates: Current use, calculations, and interpretation. J Exp Psychol Gen. 2012;141(1):2-18.

39. Pica N, Bourgeois F. Discontinuation and Nonpublication of Randomized Clinical Trials Conducted in Children. Pediatrics. 2016;138(3):e20160223-e20160223.

40. Khan KS, Hills R. Can we trust the results of trials that are stopped early? BJOG An Int J Obstet Gynaecol. 2006;113(7):766-768.

41. Schroeder AS, Von Kries R, Riedel C, et al. Patient-specific determinants of responsiveness to robot-enhanced treadmill therapy in children and adolescents with cerebral palsy. Dev Med Child Neurol. August 2014:1-8.

42. Hanna SE, Rosenbaum PL, Bartlett DJ, et al. Stability and decline in gross motor function among children and youth with cerebral palsy aged 2 to 21 years. Dev Med Child Neurol. 2009;51(4):295-302.

43. Smits D-W, Gorter JW, Hanna SE, et al. Longitudinal development of gross motor function among Dutch children and young adults with cerebral palsy: an investigation of motor growth curves. Dev Med Child Neurol. 2013;55(4):378-384.

44. Kiresuk T, Sherman R. Goal attainment scaling: A general method for evaluating comprehensive community mental health programs. Community Ment Heal J. 1968;4(6):443-453.

45. Turner-Stokes L. Goal Attainment Scaling (GAS) in Rehabilitation: A practical guide. Clin Rehabil. 2009;23(4):362-370. 
46. Nieuwenhuis JB, Irving E, Oude Rengerink K, et al. Pragmatic trial design elements showed a different impact on trial interpretation and feasibility than explanatory elements. J Clin Epidemiol. 2016;77:95-100.

47. Loudon K, Treweek S, Sullivan F, Donnan P, Thorpe KE, Zwarenstein M. The PRECIS-2 tool: Designing trials that are fit for purpose. BMJ. 2015;350.

48. Mills EJ, Chan AW, Wu P, Vail A, Guyatt GH, Altman DG. Design, analysis, and presentation of crossover trials. Trials. 2009;10:1-6.

49. Wiart L, Rosychuk RJ, Wright FV. Evaluation of the effectiveness of robotic gait training and gait-focused physical therapy programs for children and youth with cerebral palsy: a mixed methods RCT. BMC Neurol. 2016;16(1):86. 
CHAPTER 8

\section{GENERAL DISCUSSION}

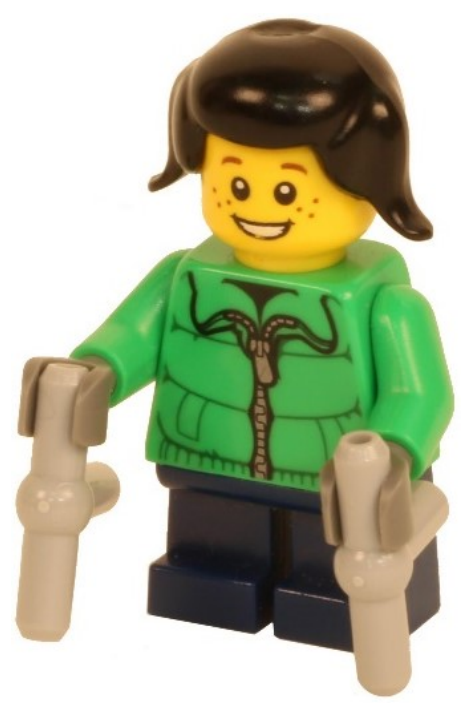


Independent mobility and daily walking activity are often restricted in children with neuromotor disorders. ${ }^{1}$ The dependency on wheelchair, walking device or an accompanying person reduces their possibilities to explore and experience their environment actively. ${ }^{2,3}$ Robot-assisted gait training (RAGT) is one example of the various therapeutic interventions that aim at improving children's walking abilities to reduce their dependency. Being implemented more than ten years ago in the pediatric neurorehabilitation setting, RAGT with the Lokomat should have come of age by now. Nevertheless, information on clear training prescriptions and optimal dosage parameters are absent, and evidence on its effectiveness in the pediatric population is weak and inconclusive from a scientific as well as a clinical perspective. An aggravating factor is that there seems to be no consensus yet on the most relevant and meaningful measures to evaluate the effectiveness of RAGT in children with neuromotor disorders.

In this general discussion, we reflect on the most important findings of this PhDthesis regarding measuring and training of walking abilities in pediatric neurorehabilitation. Furthermore, we discuss methodological considerations regarding research in this setting and provide implications for future studies and clinical practice related to the implementation of measures and training of walking abilities in children and youths with neuromotor disorders.

\section{MAIN FINDINGS}

\section{Measuring of walking abilities}

In the first part of the PhD-thesis, we focused on the measures available in research and clinical practice to assess walking abilities. Our first step was to conduct a systematic review of the evidence on the psychometric properties of gait capacity and performance measures in neuropediatric populations (chapter 2). ${ }^{4}$ We chose the International Classification of Functioning, Disability and Health for Children and Youth (ICF-CY) as a reference framework for our research as this approach illustrates that disability is a result of a complex interaction between the person and his or her environment. Accordingly, researchers should extend beyond using only 
measures of body-structure and function, but also consider the persons' functioning within their environment (i.e., performance).

Our systematic review provided an overview of the current evidence on the psychometric properties of 27 measures used to assess functional gait on the activity component of the ICF-CY in children and youths with neuromotor disorders. As children with cerebral palsy (CP) represent the most substantial fraction in the neuropediatric field, it is the diagnosis also most often considered in studies evaluating the psychometric properties of a measure in pediatric rehabilitation. Researchers and clinicians have to be aware of this fact, as pediatric neurorehabilitation generally involves a more heterogeneous group of children with several other health conditions. In contrast to our review, most other systematic reviews on mobility or activity measures exclusively focused on children with CP. ${ }^{5-}$ 13 Nevertheless, even in this specific population, evidence on measurement properties of outcome measures is still limited, let alone other health conditions in the pediatric field. Additionally, there is a focus on specific psychometric properties that are examined. While far more studies evaluated aspects of reliability, measurement error (SEM) was rarely investigated. However, information on the absolute agreement would be highly valuable for evaluative measures as it facilitates the estimation whether a change that has occurred is a real change. In the systematic review, we found some measures with strong or moderate and several measures with limited positive evidence on aspects of reliability, most of them in children with $\mathrm{CP}$.

Studies on responsiveness are even less frequent compared to studies on measurement error. However, knowledge of an outcome measure's responsiveness is vital to allow a decision on its suitability for a longitudinal evaluation as being essential for research into the effectiveness of treatment. As an outcome of the review, positive evidence on responsiveness is restricted to the Gross Motor Function Measure (GMFM) dimension E in children with Down syndrome and CP as well as the Functional Mobility Scale (FMS) in children with CP. 
Our findings are mostly in agreement with other reviews.9,10,14,15 Discrepancies regarding reported evidence levels of single tests are mainly due to differences in considering the methodological quality and the sample size of the included studies.

More studies on psychometric properties of gait measures as well as two systematic reviews have been published since the publication of our review in 2014. ${ }^{16,17}$ Both reviews also report on the validity of gait measures, thus enabling a more comprehensive view on the properties of these measures - although restricted to children with CP. While these reviews found positive evidence on aspects of reliability for some additional activity measures, the status regarding measurement error and responsiveness remained seemingly unchanged.

Our review reveals a substantial lack of evidence regarding the psychometric properties - especially regarding responsiveness and measurement error - of many measures that are commonly used to assess children's gait function in research as well as in clinical practice. Although the appraised measures cover variable constructs, most of them address the children's capacity level within the ICF-CY, while only a few consider the performance level. As performance measures account for the influence of various environments that children are exposed to in real life, this fact contrasts with the primary goal of rehabilitation, which is to empower children to live their lives as independently as possible.

Clinicians and researchers are obliged to find consensus on the most relevant and appropriate measures across all ICF-CY components and test these on their psychometric properties in either different or more heterogeneous pediatric patient populations, depending on the measures aimed areas of application. Only the availability of psychometrically sound and practical outcome measures across the whole ICF-CY ensures an overall picture of an intervention's success on a child's walking abilities.

The results of the systematic review had a significant impact on our clinical practice and our further research: Induced by the findings of our systematic review, we analyzed the situation regarding the standardized evaluation of children's gait function that perform a RAGT program on the Lokomat in our clinic. The current state revealed an underrepresentation of measures reflecting the performance construct 
of the ICF-CY. After evaluating the relevance and feasibility of the available performance measures related to our setting, we chose the FMS and the Gillette Functional Assessment Questionnaire - walking scale (FAQ), both addressing functional mobility in the daily environment, as being the most promising measures for our purposes. Although evidence on the FMS's and FAQ's psychometric properties already exists, all evidence refers to the English versions of the measures and the children's usual environment. We translated the FMS and the FAQ into German by adhering to internationally adopted guidelines, ${ }^{18}$ and examined the psychometric properties of these German versions in a population of children with neuromotor disorders within our inpatient neurorehabilitation setting.

Regarding concurrent validity, all correlation coefficients between the two measures and the chosen comparator measures, which were well known and commonly used activity measures to assess walking abilities in children, were moderate to strong and exceeded the hypothesized lower bounds (chapter 3). ${ }^{19}$ Further, correlation coefficients corresponded well with those reported in the original studies. ${ }^{20,21}$ Correlations with other performance measures were not stronger than with capacity measures (except for the FMS-500) in our study. This finding is in contrast to previous studies, which used comparable tools and found that children with similar levels of motor capacity or motor capability did not share the same levels of motor performance when walking in the home, school, or community environment. ${ }^{22,23}$ Our inpatient rehabilitation setting may have reduced the differences between walking performance and walking capacity. However, none of our correlation coefficients exceeded .80 , indicating that the FMS and FAQ provide additional information on functional mobility in the inpatient setting. Also compared with each other, they are based on slightly different constructs, which means that, if both are applied, different, complementary information can be obtained. The FMS scores revealed interesting additional information regarding the mobility levels of the children in our rehabilitation center who were actively training their gait function. Almost $50 \%$ of the included children were mobile without using a walking aid over 5 meters. This amount decreased to $41 \%$ over 50 meters and $30 \%$ over 500 meters, while $45 \%$ of the children used a wheelchair for the distance of 500 meters. This shift in the use of 
walking aids relative to walking distance or environmental setting is comparable with observations in the children's natural environment around their home and at school. ${ }^{24}$ We could further note that the FMS and FAQ do not seem to be affected by any floor or ceiling effects in our patient population, as long as one is interested in children's walking abilities.

For the investigation of the measures' reliability (chapter 4), ${ }^{25}$ we wanted to investigate several sources of variation concerning raters and environment. We were not only focusing on interrater reliability within the same environment but also on comparing data collected in two different, though very relevant, settings: the home environment of the child and the inpatient setting. This might be the reason why our findings regarding the reliability of the two measures were slightly lower than those reported by other authors. ${ }^{20,21,26}$ Interrater agreement between parents, physiotherapists, and nurses was above our predefined acceptance level for weighted kappa values of 0.6 at both the start and the end of the rehabilitation period. This fact is relevant, as it shows that the health professionals can estimate the walking performance of the children well, even at onset of the rehabilitation process, when they might have seen the child only a couple of times. As there was no difference between the rater groups, it does not seem to matter whether nurses or physiotherapists score the FMS and FAQ during an inpatient stay - irrespective of the differences regarding professional background and patient contact. Therefore, implementation of the two measures in the clinical routine could also contribute to a common language among interdisciplinary teams in inpatient rehabilitation - as well as between health professionals and parents. While kappa coefficients for the FAQ ranged between $0.69-0.77$, those for the FMS exceeded 0.8 for most of the comparisons. Thus, although different environmental settings might affect children's mobility, ${ }^{22,27,28}$ our results indicate that functional mobility performance in the inpatient setting and the child's usual environment, assessed with the FMS and the $F A Q$, correspond well with each other. Furthermore, from the parents' perspective, they apparently can reliably score their children's functional mobility at home and are valuable discussion partners for the health professionals. 
To evaluate the effectiveness of inpatient interventions on walking performance and interpret the relevance of this change in a clinical context, information on the measures' responsiveness and interpretation like minimally important change (MIC) is crucial. We, therefore, investigated the responsiveness of the FMS and FAQ during the children's inpatient stay in our neurorehabilitation center (chapter 5).

There is a considerable number of definitions and methods to analyze and calculate responsiveness. ${ }^{29,30}$ Some researchers interpret methods for expressing the magnitude of change over time like effect sizes and paired t-tests as measures of responsiveness. However, these methods are measuring the magnitude and not the validity of the change scores. According to the definition of the COnsensus-based Standards for the selection of health Measurement INstruments (COSMIN) group, responsiveness addresses the latter aspect. ${ }^{31}$ Thus, methodological principles for measuring responsiveness should be the same as for validity studies, with the difference that the focus is on change scores collected in a longitudinal study design over time. We applied two different approaches, a construct and a criterion approach, both recommended by the COSMIN group. ${ }^{31}$ For the construct approach, we used comparator instruments and tested a priori formulated hypotheses on the correlations of the measures' change scores. For the criterion approach, we used the therapists' perception of whether and how the children had changed regarding their everyday walking abilities as an external anchor. With that, the results regarding the measures' change scores in comparison with the chosen external standards focused on the validity of the change scores and not on the change scores' magnitude as being the result of the treatment interventions that our children had gone through during the study period. ${ }^{29}$

The values of the area under the receiver operating characteristic (ROC) curves (AUC) derived from the criterion approach provided a helpful tool to demonstrate the FMS's and FAQ's ability to accurately discriminate between children who did or did not improve their everyday walking performance during inpatient rehabilitation according to the therapists' view.

With the anchor-based approach that we applied, we obtained a definition of what the therapists consider as minimally important change. ${ }^{32}$ This knowledge allows an 
interpretation and estimation of the clinical relevance of the measures' observed change scores after inpatient gait interventions - in clinical practice and research; a piece of information that is mostly missing in other tools.

Our results demonstrate that the FMS and FAQ are not only valid to assess functional mobility in children's usual environment, but also in the inpatient setting. As performance measures, they are particularly meaningful for the child and the family, because their perspective reflects the child's behavior in everyday life. However, to be of real importance, the scores of FMS and FAQ assessed in the inpatient neurorehabilitation setting should be in agreement with those measured in the child's usual environment. Among others, we could answer this question positively in our reliability study. This finding is a crucial prerequisite for the communication between parents and health care professionals when working together on the rehabilitation process of the child. With the positive results regarding the measures' responsiveness as well as the information available on the MIC, the FMS and FAQ are now among the few pediatric functional mobility instruments with information on each psychometric property. With that, they enable clinicians and researchers to adequately evaluate interventions in pediatric neurorehabilitation regarding their effect on children's functional mobility on the ICF-CY construct of performance. This is also meaningful for the involved families, since relevant aspects of their everyday life are covered. Another positive aspect is that the measures' psychometric properties are not restricted to children with $\mathrm{CP}$, but apply to a very heterogeneous group of children with neuromotor disorders as being the standard population of our rehab center. Hence, we can recommend their usage for settings that are comparable to ours. Still, further trials that provide information on the intrarater reliability and smallest detectable change of the FMS and FAQ, or examine the measures' psychometric properties in subgroups related to age, functional abilities, or living environment would help raise the evidence to a higher level with more specific applicable thresholds per subgroup.

\section{Training of walking abilities}

In the second part of this PhD-thesis, we focused on the training aspect of walking abilities in children with spastic CP. Chapters 6 and 7 describe the protocol, 
realization, and findings of our pragmatic, randomized, crossover trial, in which we aimed to investigate the effectiveness of RAGT with the Lokomat compared to usual care in ambulatory children with spastic CP. ${ }^{33}$ We could not confirm our hypothesis that five weeks of RAGT were superior regarding the improvement of walking abilities compared to five weeks of usual care.

Existing evidence on the effectiveness of RAGT with the Lokomat in children is weak and inconsistent. ${ }^{34}$ Only four randomized controlled trials (RCTs) have been published, all restricted to children with CP with a GMFCS level I to III. Two of these studies demonstrated improvements in GMFM dimension E scores, ${ }^{35,36}$ while results regarding kinematic and temporospatial parameters are conflicting: Two studies found no effects, ${ }^{36,37}$ whereas another research group - with considerable limitations regarding their statistical procedures - showed significant improvements in the kinematics of the lower and upper extremities in their children. ${ }^{35,38}$ Lower-level designed studies yielded inconsistent results as well, regarding improvements in gait speed, gait endurance, and gross motor function. ${ }^{34}$

Reflecting on our results and experiences of conducting the trial over the past years, we have learned a lot from both a content-related and a methodological point of view. As we wanted to gain relevant and transferable information for the clinical setting, we aimed for a pragmatic trial design. A study is never either purely explanatory or pragmatic but moves within a continuum in relation to different trial aspects. ${ }^{39}$ It has been shown that applying explanatory elements into a pragmatic trial and vice versa can affect validity, generalizability, and feasibility. 40

Figure 8.1. illustrates how we assess our study today in relation to various parameters on the explanatory-pragmatic continuum with the PRECIS-2 tool that is now available. ${ }^{39}$ This tool was specifically designed to support researchers in their choices regarding the explanatory-pragmatic continuum when developing a trial protocol. 


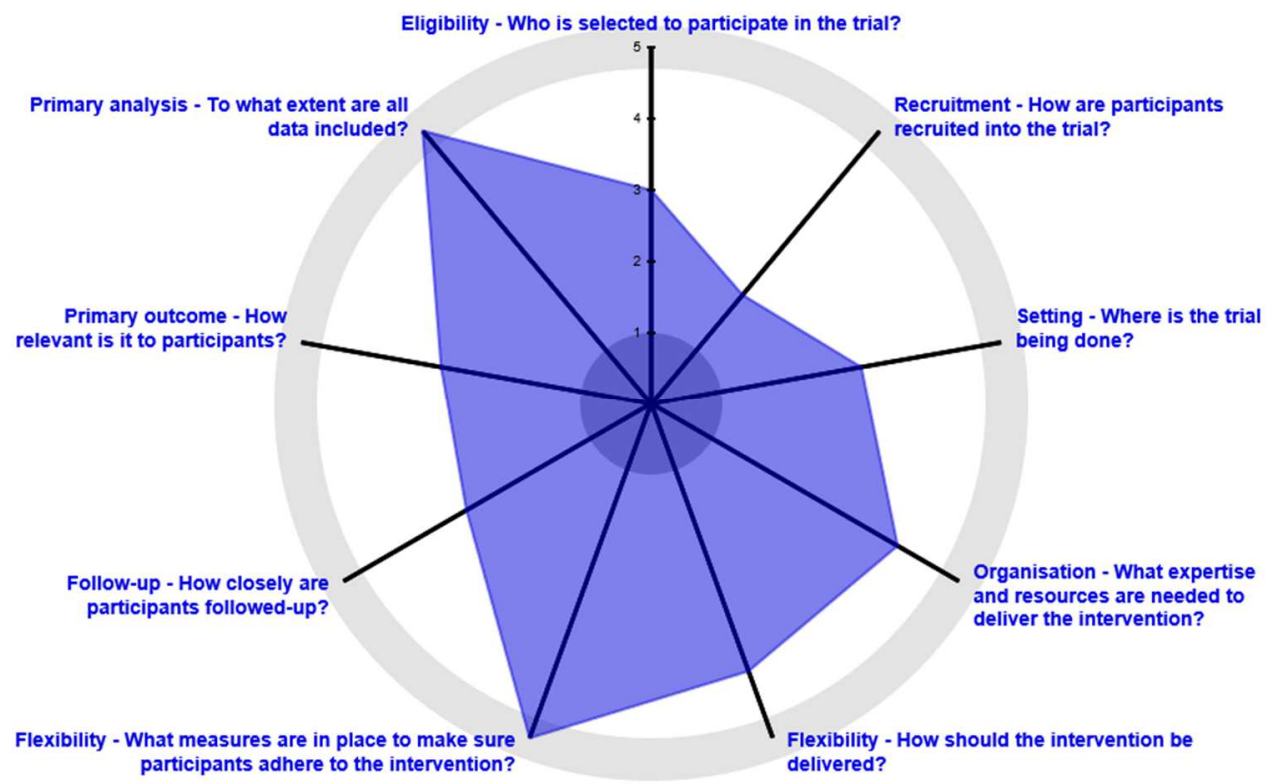

Figure 8.1. The PRagmatic-Explanatory Continuum Indicator Summary - PRECIS-2 wheel.

The PRECIS-2 wheel visualizes the pragmatic/explanatory extent of a study design. Nine domains are scored on a scale from 1 (very explanatory) to 5 (very pragmatic). The closer to the rim the wheel is the more pragmatic is the approach of a study.

In retrospect, some choices that we made towards the explanatory side in the planning phase of the study subsequently turned out to be aggravating factors in the course of the study. Related to the content of the rehabilitation process based on the ICF-CY, it might be a better and more pragmatic choice to compare RAGT as it is currently predominantly applied in our clinical setting as an add-on therapy element within the whole treatment package. On the one hand, this implies a multimodal and multidisciplinary approach - which, for example, involves the transfer of changes in body functions into activities of daily living. This approach would also enable us to recruit patients within our usual clinical setting - a more pragmatic choice than the one in our study, which would have facilitated recruitment largely. On the other hand, the pragmatic approach could also be reflected in the outcome measures used. In this respect, performance measures and individualized tools that address the personal goals of the participants and their families are particularly useful and thus influence the study results' relevance. 


\section{METHODOLOGICAL CONSIDERATIONS}

\section{Measuring of walking abilities}

We defined the scope of our systematic review based on the measures' most essential requirements regarding their potential utilization in our rehabilitation center. Therefore, we decided to consider measures related to any pediatric neuromotor disorder, but to limit the review on reliability, measurement error, and responsiveness, as these are the most critical psychometric properties for an evaluative outcome measure. Another review that appraises validity aspects of the available measures, while considering all pediatric neuromotor disorders, would be desirable to complete the picture regarding the available evidence.

Definitions, criteria, and explanations provided by the COSMIN group provided an invaluable framework for our systematic review as well as all psychometric studies. ${ }^{31,41,42}$ The COSMIN standards were also very beneficial in the further course of this $\mathrm{PhD}$-thesis to ensure a high methodological quality of our psychometric studies. Additionally, adhering to these criteria improves the comparability and ability to summarize the results of various studies.

By introducing the FMS and FAQ in the inpatient setting to score children's inpatient functional mobility performance, we had to adapt the rating procedure to a certain extent. Thus, we chose to have the scoring done by the physiotherapists and the nurses to determine additionally a group of health professionals most qualified to score everyday life mobility in the inpatient setting. While we could apply the FAQ as it was, we had to customize the explanations of the three FMS-items to our inpatient setting. Accordingly, we defined the FMS- 5 as the child's mobility in the patient room, the FMS-50 as mobility within the rehabilitation center (between wards, therapies, and inpatient school), and the FMS-500 as mobility outside around the rehabilitation center. High kappa coefficients and high agreement between our health professionals, who scored everyday life mobility in the rehabilitation setting, and parents, who rated their child's mobility in the usual environment, provided evidence that the adaptations made for the inpatient setting seem to correspond well to the home setting. 
Due to our inpatient setting, we had to make some concessions regarding the administration mode of the FMS and FAQ. Nurses and physiotherapists scored the FMS and FAQ based on their observations instead of parents- or child-interview. This ensured a rating that referred to the children's gait performance in the inpatient setting. Standardized interviews with the health professionals facilitated that they scored the measures regarding performance and not capacity, which is much more common, especially among therapists. Our approach might have influenced the correlation strength between the FMS/FAQ and the comparators, as well as increased the interrater reliability of the measures.

For the validity and responsiveness studies, we compared the FMS and FAQ with other measures of walking ability. Despite the missing evidence on the psychometric properties of the measures' subcategories, we used the dimension E of the GMFM and the walking item of the WeeFIM instead of the comprehensive measures as comparators. Although being aware of the limitations, we preferred this option to the use of multidimensional instruments with known psychometric properties as this choice ensured a similar underlying construct in the index and comparator measures. The same restrictions also relate to the GRS that we applied as an anchor for the evaluation of the measures' MIC: although the psychometric properties of such a single question assessing change may be unknown, it often has high face validity. ${ }^{32}$ Regarding the MIC, we consider a GRS estimation through parents or children as most relevant. However, we had the GRS rated by the therapists due to the same aspects related to our setting as mentioned for the rating of the FMS and FAQ. Discussions are going on, whether a GRS should be used within a criterion or a construct approach for the assessment of responsiveness. We, therefore, applied both methods.

The study samples of the psychometric studies mainly represented the typical patient population of our clinical inpatient rehabilitation setting and were very heterogeneous regarding age, diagnoses, mobility levels, and rehabilitation duration. Whereas this heterogeneity was desirable from a content-related perspective, it resulted in variable numbers regarding the comparator tests, as not all included patients were able to perform all tests at each measurement time point. 


\section{Training of walking abilities}

We only recruited sixteen of the anticipated 34 patients in our trial on the effectiveness of RAGT, despite prolonging the study duration, broadening the inclusion criteria, and expanding the mono- to a bi-center trial after five years. Reasons for our recruitment difficulties are diverse. Although we had used various channels to promote our study, the response rate was low. The logistical challenges of reconciling the study protocol with the families' requirements of everyday life and the burden on resources of the rehabilitation center were huge. Travel efforts due to the decentralized location of our rehabilitation center limited study participation to school holiday periods for most families. The second study center could not contribute additional children either because of the same reasons, despite the local study coordinator's a priori estimation of two to three study participants per year.

Although we restricted study participation to children with spastic CP with a GMFCS level II-IV who can cover at least 14 meters, our sample was still very heterogeneous regarding age, body function level, cognitive function, and walking abilities, which reflects the heterogeneity of this patient group. Children also differed regarding their previous RAGT experience. In everyday clinical practice, children undergoing RAGT training in our clinic are even more heterogeneous, since we also treat children with diagnoses other than $\mathrm{CP}$, who may also benefit from a spontaneous neurological recovery, for example.

Over the long duration of our trial, methods and principles of RAGT application and accompanying software, as well as serious game possibilities, have changed considerably. Owing to the pragmatic trial design, we always applied the current state-of-the-art RAGT strategies which best suited the children's capabilities. We continually implemented the latest Lokomat software and serious games, which, in addition to the motivation provided by the therapists, facilitated as active child participation as possible. We also adjusted the training parameters (body-weight support, gait speed, guidance force, and range of motion) individually to each child. While this approach warranted that each child received the most optimal treatment, it rules out the possibility to deduce the most effective RAGT set-up. 
The conceptualization of the trial started more than nine years ago. At this time, we concentrated on outcome measures that were often used in other studies on gait training in children with neuromotor disorders. These had been mainly measures of body function and walking capacity, most of them with limited evidence on their psychometric properties. Measures of walking performance had not yet been available in German. The question arises whether the results would have been different if we had been able to implement the FMS and FAQ in our trial.

We chose a crossover study design for our RCT to deal with the problem of the small sample sizes, which are common in pediatric clinical research. This design has the advantage that each child can act as their own control, which results in a higher precision regarding the estimation of the treatment effect and thus reduces the necessary sample size..$^{43}$ Although sample sizes are reduced compared to the standard parallel-group design, the crossover design is not the answer to all problems, as sample sizes needed can still be too high for pediatric intervention studies. Furthermore, duration of the trial is increased because every patient has to complete at least two study periods. ${ }^{44} \mathrm{~A}$ further disadvantage of the design is the risk of carryover effects, which our study was exposed to because of the omission of a washout phase. Statistical analyses did not indicate any reason to suspect carryover effects, though.

We chose a 5-week follow-up period, which is on the one hand somewhat short, but could decrease the influence of confounding factors to some extent (e.g., growth, illness, motor development) on the other side. The question of the optimal follow-up period is especially tricky to be answered in the pediatric field, where the influence of growth and developmental changes are added to common factors as changes in medication or concomitant therapies, psychosocial aspects, illnesses, work load, etc.

\section{IMPLICATIONS FOR FUTURE RESEARCH AND CLINICAL PRACTICE}

This $\mathrm{PhD}$-thesis addresses the measurement and training of functional walking abilities - two fundamentally different aspects, which are nonetheless firmly interrelated: Results of an intervention trial are only valuable for clinical practice and 
research if sound outcome measures have been applied that are relevant for the field in question.

The arrival of the first Lokomat in our rehabilitation center in 2005 was simultaneously the birth of our research group. Being the first center worldwide that applied RAGT with the Lokomat in children, evidence on its feasibility, safety, and effectiveness was apparently nonexistent. We, therefore, implemented standardized assessment procedures and monitored the clinical RAGT training scientifically. ${ }^{45-49}$ However, our results always derived from children who were involved in a multimodal and interdisciplinary inpatient rehabilitation program, which we did not duly take into account when interpreting the results. Against this background, we started in 2009 our RCT on an outpatient basis to close part of the knowledge gap regarding the effectiveness of RAGT in children. ${ }^{33}$

During the course of the trial, we realized more and more the significance of appropriate and meaningful outcome measures. Body function measures, for example, may not be of high importance for young children with disabilities, as they refer to their usual walking pattern as "normal" and do not necessarily aim to walk in so-called typical gait patterns. ${ }^{50}$ Our systematic review on gait-related activity measures showed us that many tools that are used in clinical routine and research have no or only very limited evidence on their psychometric properties. Furthermore, also the relevance of these measures for the children or their families is often questionable. In contrast, performance measures allow a direct transfer regarding the child's activity in its everyday life environment and therefore deserve special consideration. They have an integral value in the evaluation of children's walking abilities by offering further insights into the complex construct of functional mobility. These aspects motivated us to select two performance measures that we considered relevant in our setting, translate them into German and test their psychometric properties in the inpatient setting for eventual implementation in our clinical routine.

By comparing the FMS and FAQ with other frequently used gait measures, we could show that they provide additional information. ${ }^{19}$ This information enables the multidisciplinary rehabilitation team to get a more comprehensive overview of a 
child's functional walking abilities and to use a common language regarding the child's functional mobility in everyday clinical routine.

Also, the fact that the relationship between children's capacity and participation in life seems to be significantly mediated by their performance hints at the relevance of including performance tools into the clinical assessment battery. ${ }^{51}$

Our psychometric studies on the FMS and FAQ demonstrated their scores' correspondence between the inpatient setting and the child's usual environment, their responsiveness to change, and yielded first knowledge on their MICs regarding inpatient rehabilitation. ${ }^{25}$ With these prerequisites, FMS and FAQ would have been ideal outcome measures in our RCT. Used together, they would have enabled a comprehensive assessment of functional mobility in the children's everyday life by taking into account walking aids, different settings, as well as various terrains. Unfortunately, they were not yet available in German at the start of our RCT. The question remains open whether the outcome of our trial might have been different with these now available tools.

In the meantime, we implemented the FMS and FAQ in the clinical assessment routine of our rehabilitation center. Physiotherapists score the walking performance of all children performing any gait training in our center at the beginning and the end of rehabilitation as standard. To facilitate extensive use of the measures in the German-speaking area, we provide the downloadable German versions of the two performance measures on our website for free (https://www.kispi.uzh.ch/rza/de/forschende/publikationen/downloads/) - by courtesy of the original developers. Future research could address the question, whether one or both measures could also be suitable as a classification instrument of children's functional mobility. So far, we only know of the GMFCS as such a tool, which - although widely used for children with different diagnoses - is only valid for children with CP. ${ }^{52}$

Being aware of the small patient populations in our research area, we chose a crossover study design for our RCT. Although this study design reduces the necessary number of study participants compared to a parallel study design, it still turned out to be not the optimal solution for our research field. Thus, an important 
question that has to be answered for further prospective intervention studies in our area is, whether other problem-solving approaches would be more promising.

One possibility concerning the study design might be to use alternative research approaches, e.g., an n-of-1 trial or a single case methodology design. N-of-1 and single case methodology trials evaluate different treatment effects within a single patient over time. ${ }^{53,54} \mathrm{~N}$-of-1 trials use multiple (usually randomized and blinded) crossover periods, ${ }^{55}$ whereas single case methodology studies apply only one crossover period with three to five measurements per phase ${ }^{54}$ Different types of nof-1 trials exist, which differ in the way the intervention sequences are applied. Depending on the design that is used, results document clinical change in patient performance over time. ${ }^{56}$ By providing practical information, $n$-of- 1 and single case methodology trials facilitate the decision-making process for clinicians. With that, they are of immediate benefit for the individual patient and the clinician. Furthermore, their pragmatic design enables clinicians to contribute actively to the research in their field of knowledge. ${ }^{56} \mathrm{~A}$ considerable drawback, especially of the n-of-1 trial, is the amount of measurement time points needed. To be a feasible option, the design has to be combined with relevant outcome measures that are the least labor-, time-, and cost-intensive as possible. ${ }^{57}$ In this context, performance measures like the FMS, $F A Q$, or wireless activity monitoring (provided they exhibit adequate psychometric properties) seem to meet many of these requirements.

Another alternative approach regarding the study design could be the use of the GMFM Evolution Ratio. ${ }^{58}$ This ratio equals the measured GMFM-66 change score during a specific period divided by the expected natural evolution for the same period. For this model, data for the natural evolution were taken from the five GMFCS curves of GMFM-66 scores that were established by Rosenbaum et al. ${ }^{59}$ For studies without a control group, the model provides a possibility for the interpretation of GMFM-66 change scores in children with CP. However, this approach has some limitations, especially for studies from European countries: The data of Rosenbaum et al. are based on children with $\mathrm{CP}$ receiving standard care as provided in the nineties in Ontario, Canada. Furthermore, the GMFM Equation Ratio is only valuable 
for children between one and eight years old, and should only be used on the groupand not on the individual level. ${ }^{58}$

A third approach could consist of changes regarding the statistical perspective from a frequentist to a Bayesian framework. A Bayesian perspective offers the possibility to learn from evidence as it accumulates by combining prior knowledge that usually derives from previous comparable research with information of the current study. ${ }^{60}$ In this way, inferences are updated with each new information that is available. This integration of prior knowledge could decrease the sample size needed in the current trial. ${ }^{61}$ As the Bayesian approach provides a direct probability interpretation, the interpretation of the results is also more intuitive for clinicians. ${ }^{62}$ Of course, there are also limitations to this statistical approach, the most common criticism being the possibility of deciding on suitable prior information, for example. ${ }^{60}$

Besides the possibilities regarding study design or statistical methods, an inpatient approach may be a more promising strategy from the content side to overcome our recruitment difficulties and would additionally allow an increase of the frequency or duration of the RAGT intervention. It would also offer the possibility to add conventional gait therapy sessions, in which the therapists could instruct and help the children to adopt the movement experience during RAGT into their on-ground walking. This combination of therapy on the body function component (RAGT) and its translation into activities (conventional therapies) is already being used and propagated in the clinical routine of our inpatient children - although evidence of its effectiveness is also pending.

Furthermore, before starting another trial on the effectiveness of RAGT in an inpatient pediatric setting, it would be worthwhile to implement a qualitative phase in which various aspects of intervention and assessments, recruitment requirements and barriers, as well as motivation to participate are discussed with affected families in question.

\section{CONCLUSION}

This PhD-thesis revealed the limited evidence on psychometric properties of gaitrelated activity measures in children with neuromotor disorders. Especially 
measurement error and responsiveness are rarely addressed, despite the fact that many research projects focus on the effectiveness of interventions. We closed this gap regarding two measures of functional mobility, the FMS and FAQ, in the pediatric inpatient setting and showed that these two measures are valid, reliable and responsive to change to assess children's functional mobility performance in the inpatient setting. Besides, our research facilitates the interpretation of patients' change scores, since we used an external anchor to assess the change from a clinical point of view.

Our pragmatic, randomized, crossover trial indicated that 15 sessions of RAGT in a 5-week period are not effective in improving walking abilities in ambulatory children with spastic CP. With these findings, evidence on the effectiveness of RAGT in the pediatric population remains weak. A future study should preferably start with a qualitative phase which inquires about the families perspective to overcome recruitment difficulties, extend over a longer RAGT duration, include individualized outcome measures that also reflect the performance construct of the ICF-CY, and the researchers should consider applying innovative research designs.

\section{REFERENCES}

1. Logan SW, Ross SM, Schreiber MA, et al. Why We Move: Social Mobility Behaviors of Non-Disabled and Disabled Children across Childcare Contexts. Front Public Heal. 2016;4(September).

2. van den Berg-Emons R, Saris W, de Barbanson D, Westerterp K, Huson A, van Baak M. Daily physical activity of schoolchildren with spastic diplegia and of healthy control subjects. J Pediatr. 1995;127(4):578-584.

3. Bjornson K, Belza B, Kartin D, R L, McLaughlin J. Ambulatory physical activity performance in youth with cerebral palsy and youth who are developing typically. Phys Ther. 2007;87(3):248-257.

4. Ammann-Reiffer C, Bastiaenen CHG, de Bie RA, van Hedel HJA. Measurement Properties of Gait-Related Outcomes in Youth With Neuromuscular Diagnosis: A systematic review. Phys Ther. 2014;94(8):1067-1082.

5. Sakzewski L, Boyd R, Ziviani J. Clinimetric properties of participation measures for 5- to 13-year-old children with cerebral palsy: a systematic review. Dev Med Child Neurol. 2007;49(3):232-240. 
6. Carlon S, Shields N, Yong K, Gilmore R, Sakzewski L, Boyd R. A systematic review of the psychometric properties of Quality of Life measures for school aged children with cerebral palsy. BMC Pediatr. 2010;10(1):81.

7. Ketelaar M, Vermeer A, Helders PJ. Functional motor abilities of children with cerebral palsy: a systematic literature review of assessment measures. Clin Rehabil. 1998;12(5):369-380.

8. Baird MW, Vargus-Adams J. Outcome measures used in studies of botulinum toxin in childhood cerebral palsy: a systematic review. J Child Neurol. 2010;25(6):721-727.

9. Harvey A, Robin J, Morris ME, Graham HK, Baker R. A systematic review of measures of activity limitation for children with cerebral palsy. Dev Med Child Neurol. 2008;50(3):190-198.

10. Balemans AC, Fragala-Pinkham $\mathrm{M}$ a, Lennon $\mathrm{N}$, et al. Systematic review of the clinimetric properties of laboratory- and field-based aerobic and anaerobic fitness measures in children with cerebral palsy. Arch Phys Med Rehabil. 2013;94(2):287301.

11. Debuse $\mathrm{D}$, Brace $\mathrm{H}$. Outcome measures of activity for children with cerebral palsy: a systematic review. Pediatr Phys Ther. 2011;23(3):221-231.

12. Morris C, Kurinczuk JJ, Fitzpatrick R. Child or family assessed measures of activity performance and participation for children with cerebral palsy: a structured review. Child Care Heal Dev. 2005;31(4):397-407.

13. Oeffinger D, Bagley a, Rogers $S$, et al. Outcome tools used for ambulatory children with cerebral palsy: responsiveness and minimum clinically important differences. Dev Med Child Neurol. 2008;50(12):918-925.

14. Adair B, Said CM, Rodda J, Morris ME. Psychometric properties of functional mobility tools in hereditary spastic paraplegia and other childhood neurological conditions. Dev Med Child Neurol. 2012;54(7):596-605.

15. Bartels B, de Groot JF, Terwee CB. The Six-Minute Walk Test in Chronic Pediatric Conditions: A Systematic Review of Measurement Properties. Phys Ther. 2013;93(4):529-541.

16. Himuro N, Abe H, Nishibu H, Seino T, Mori M. Easy-to-use clinical measures of walking ability in children and adolescents with cerebral palsy: a systematic review. Disabil Rehabil. 2017;39(10):957-968.

17. Zanudin A, Mercer TH, Jagadamma KC, van der Linden ML. Psychometric properties of measures of gait quality and walking performance in young people with Cerebral Palsy: A systematic review. Gait Posture. 2017;58(June):30-40.

18. Beaton DE, Bombardier C, Guillemin F, Ferraz MB. Guidelines for the Process of Cross-Cultural Adaptation of Self-Report Measures. Spine (Phila Pa 1976). 2000;25(24):3186-3191. 
19. Ammann-Reiffer C, Bastiaenen CHG, Klöti C, van Hedel HJA. Concurrent Validity of Two Gait Performance Measures in Children with Neuromotor Disorders. Phys Occup Ther Pediatr. 2018;2638:1-12.

20. Novacheck TF, Stout JL, Tervo R. Reliability and Validity of the Gillette Functional Assessment Questionnaire as an Outcome Measure in Children with Walking Disabilities. J Pediatr Orthop. 2000;20(1):75-81.

21. Graham HK, Harvey A, Rodda J, Nattrass GR, Pirpiris M. The Functional Mobility Scale (FMS). J Pediatr Orthop. 2004;24(5):514-520.

22. Tieman BL, Palisano RJ, Gracely EJ, Rosenbaum PL. Gross Motor Capability and Performance of Mobility in Children With Cerebral Palsy: A Comparison Across Home, School, and Outdoors/Community Settings. Phys Ther. 2004;84:419-429.

23. Holsbeeke L, Ketelaar M, Schoemaker MM, Gorter JW. Capacity, capability, and performance: different constructs or three of a kind? Arch Phys Med Rehabil. 2009;90(5):849-855.

24. Harvey A, Baker R, Morris ME, Hough J, Hughes M, Graham HK. Does parent report measure performance? A study of the construct validity of the Functional Mobility Scale. Dev Med Child Neurol. 2010;52(2):181-185.

25. Ammann-Reiffer C, Bastiaenen CHG, de Bie RA, van Hedel HJA. Interrater Reliability of Two Gait Performance Measures in Children with Neuromotor Disorders Across Two Different Settings. Dev Med Child Neurol. 2017;59(11):11581163.

26. Harvey AR, Morris ME, Graham HK, Wolfe R, Baker R. Reliability of the functional mobility scale for children with cerebral palsy. Phys Occup Ther Pediatr. 2010;30(2):139-149.

27. Palisano RJ, Tieman BL, Walter SD, et al. Effect of environmental setting on mobility methods of children with cerebral palsy. Dev Med Child Neurol. 2003;45(2):113-120.

28. Palisano RJ, Hanna SE, Rosenbaum PL, Tieman B. Probability of walking, wheeled mobility, and assisted mobility in children and adolescents with cerebral palsy. Dev Med Child Neurol. 2010;52(1):66-71.

29. Husted J a, Cook RJ, Farewell VT, Gladman DD. Methods for assessing responsiveness: a critical review and recommendations. J Clin Epidemiol. 2000;53(5):459-468.

30. Terwee C, Dekker F, Wiersinga W, Prummel M, Bossuyt P. On assessing responiveness of health-related quality of life instruments: Guidelines for instrument evaluation. Qual Life Res. 2003;12(4):349-362.

31. Mokkink LB, Terwee CB, Patrick DL, et al. The COSMIN study reached international consensus on taxonomy of measurement properties for HRPR outcomes. J Clin Epidemiol. 2010;63:737-745. 
32. de Vet HCW, Terwee CB, Mokkink LB, Knol DL. Measurement in Medicine. Cambridge: Cambridge University Press; 2011.

33. Ammann-Reiffer C, Bastiaenen CHG, Meyer-Heim AD, van Hedel HJA. Effectiveness of robot-assisted gait training in children with cerebral palsy: $A$ bicenter, pragmatic, randomized, cross-over trial (PeLoGAIT). BMC Pediatr. 2017;17(1).

34. Lefmann S, Russo R, Hillier S. The effectiveness of robotic-assisted gait training for paediatric gait disorders: systematic review. J Neuroeng Rehabil. 2017;14(1):1.

35. Wallard L, Dietrich G, Kerlirzin Y, Bredin J. Robotic-assisted gait training improves walking abilities in diplegic children with cerebral palsy. Eur $J$ Paediatr Neurol. 2017;21(3):557-564.

36. Peri E, Turconi A, Biffi E, et al. Effects of dose and duration of Robot-Assisted Gait Training on walking ability of children affected by cerebral palsy. Technol Heal Care. 2017;9(4):671-681.

37. Druzbicki M, Rusek W, Snela $S$, et al. Functional effects of robotic-assisted locomotor treadmill therapy in children with cerebral palsy. J Rehabil Med. 2013;45(4):358-363.

38. Wallard L, Dietrich G, Kerlirzin Y, Bredin J. Effect of robotic-assisted gait rehabilitation on dynamic equilibrium control in the gait of children with cerebral palsy. Gait Posture. 2017;11(60):55-60.

39. Loudon K, Treweek S, Sullivan F, Donnan P, Thorpe KE, Zwarenstein M. The PRECIS-2 tool: Designing trials that are fit for purpose. BMJ. 2015;350.

40. Nieuwenhuis JB, Irving E, Oude Rengerink K, et al. Pragmatic trial design elements showed a different impact on trial interpretation and feasibility than explanatory elements. J Clin Epidemiol. 2016;77:95-100.

41. Mokkink LB, Terwee CB, Knol DL, et al. The COSMIN checklist for evaluating the methodological quality of studies on measurement properties: A clarification of its content. BMC Med Res Methodol. 2010;10(22).

42. Terwee CB, Mokkink LB, Knol DL, Ostelo RWJG, Bouter LM, de Vet HCW. Rating the methodological quality in systematic reviews of studies on measurement properties: a scoring system for the COSMIN checklist. Qual Life Res. 2012;21(4):651-657.

43. Senn S. Cross-over Trials in Clinical Research. 2nd ed. Chichester: John Wiley \& Sons Ltd.; 2002.

44. Wellek S, Blettner M. On the Proper Use of the Crossover Design in Clinical Trials. Dtsch Arztebl Int. 2012;109(15):276-281. 
45. Meyer-Heim A, Borggraefe I, Ammann-Reiffer C, et al. Feasibility of roboticassisted locomotor training in children with central gait impairment.pdf. Dev Med Child Neurol. 2007;49:900-906.

46. Borggraefe I, Meyer-Heim A, Kumar A, Schaefer J, Berweck S, Heinen F. Improved gait parameters after robotic-assisted locomotor treadmill therapy in a 6year-old child with cerebral palsy. Mov Disord. 2008;23(2):280-283.

47. Meyer-Heim A, Ammann-Reiffer C, Schmartz A, et al. Improvement of walking abilities after robotic-assisted locomotion training in children with CP. Arch Dis Child. 2009;94(8):615-620.

48. Borggraefe I, Schaefer JS, Klaiber M, et al. Robotic-assisted treadmill therapy improves walking and standing performance in children and adolescents with cerebral palsy. Eur J Paediatr Neurol. 2010;14(6):496-502.

49. Borggraefe I, Klaiber M, Schuler T, et al. Safety of robotic-assisted treadmill therapy in children and adolescents with gait impairment: a bi-centre survey. Dev Neurorehabil. 2010;13(2):114-119.

50. Phelan SK, Gibson BE, Wright FV. What is it like to walk with the help of a robot? Children's perspectives on robotic gait training technology. Disabil Rehabil. 2015;8288(November):1-10.

51. Bjornson KF, Zhou C, Stevenson R, Christakis DA. Capacity to participation in cerebral palsy: Evidence of an indirect path via performance. Arch Phys Med Rehabil. 2013;94(12):2365-2372.

52. Towns M, Rosenbaum P, Palisano R, Wright F. Should the Gross Motor Function Classification System be used for children who do not have cerebral palsy? Dev Med. 2018;60(2):147-154.

53. Duan N, Kravitz RL, Schmid CH. Single-patient (n-of-1) trials: A pragmatic clinical decision methodology for patient-centered comparative effectiveness research. $J$ Clin Epidemiol. 2013;66(8 SUPPL.8):1-12.

54. Tate RL, Perdices M, Rosenkoetter $U$, et al. The Single-Case Reporting Guideline In BEhavioural Interventions (SCRIBE) 2016: Explanation and elaboration. Arch Sci Psychol. 2016;4(1):10-31.

55. Kravitz RL, Duan N, eds, and the DEcIDE Methods Center N-of-1 Guidance Panel (Duan N, Eslick I, Gabler NB, Kaplan HC, Kravitz RL, Larson EB, Pace WD, Schmid CH, Sim I VS. Design and Implementation of N-of-1 Trials: A User's Guide. AHRQ Publi. Rockville, MD: Agency for Healthcare Research and Quality; 2014.

56. Graham JE, Karmakar AM, Ottenbacher KJ. Small Sample Research Designs for Evidence-based Rehabilitaton: Issues and Methods. Arch Phys Med Rehabil. 2012;93(8):1-13.

57. O Lillie E, Patay B, Diamant J, Issell B, Topol EJ, Schork NJ. The n-of-1 clinical trial: the ultimate strategy for individualizing medicine? Per Med. 2011;8(2):161-173. 
58. Marois P, Marois M, Pouliot-Laforte A, Vanasse M, Lambert J, Ballaz L. Gross Motor Function Measure Evolution Ratio: Use as a Control for Natural Progression in Cerebral Palsy. Arch Phys Med Rehabil. 2016;97(5):807-814.

59. Rosenbaum PL, Walter SD, Hanna SE, et al. Prognosis for gross motor function in cerebral palsy: Creation of motor development curves. JAMA. 2002;288(11):13571363.

60. Van de Schoot R, Kaplan D, Denissen J, Asendorpf JB, Neyer FJ, van Aken MAG. A Gentle Introduction to Bayesian Analysis: Applications to Developmental Research. Child Dev. 2014;85(3):842-860.

61. FDA. Guidance for the use of Bayesian statistics in medical device clinical trials. Guid Ind FDA Staff. 2010:1-50.

62. Wong AYL, Warren S, Kawchuk GN. A new statistical trend in clinical research - Bayesian statistics. Phys Ther Rev. 2010;15(5):372-381. 
ADDENDUM

\author{
APPENDIX \\ SUMMARY
}

ZUSAMMENFASSUNG

VALORISATION

ACKNOWLEDGMENTS

ABOUT THE AUTHOR

LIST OF PUBLICATIONS

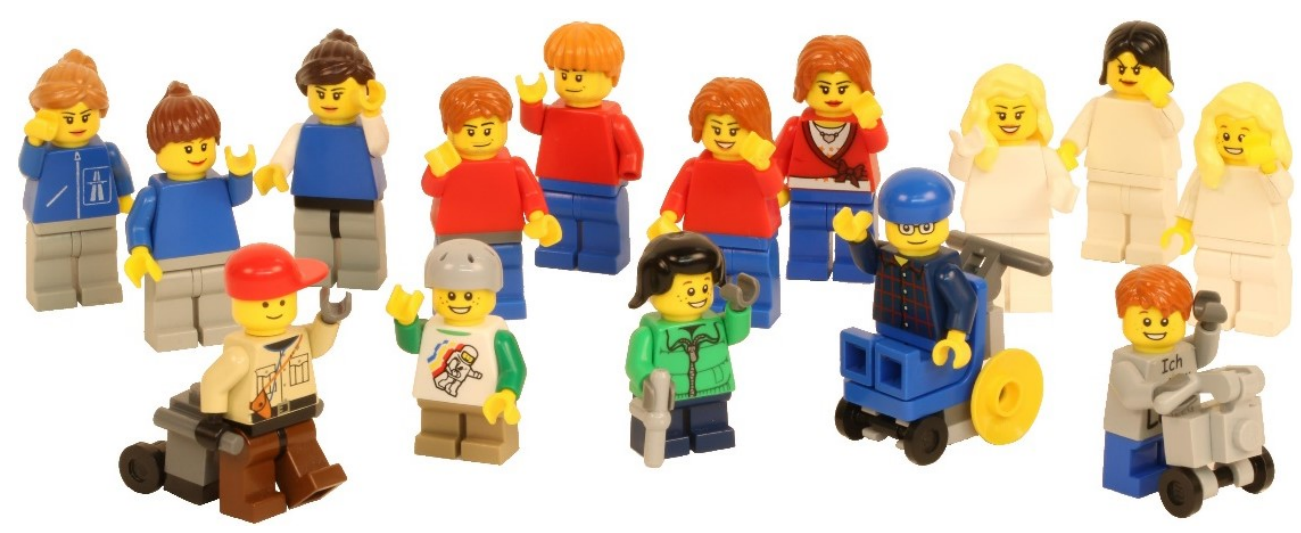


APPENDIX 


\section{GERMAN FUNCTIONAL MOBILITY SCALE}
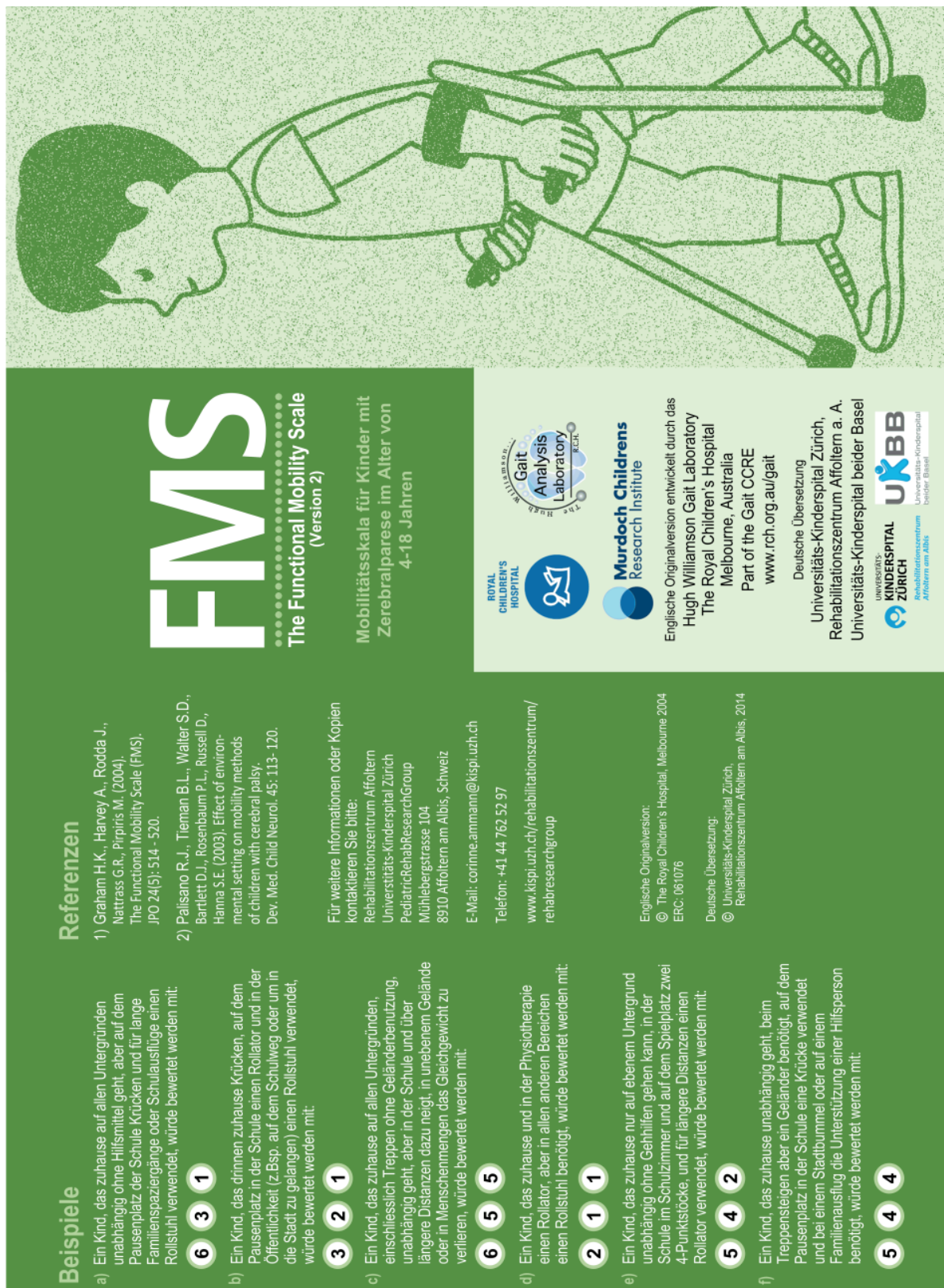
GERMAN FUNCTIONAL MOBILITY SCALE (continued)
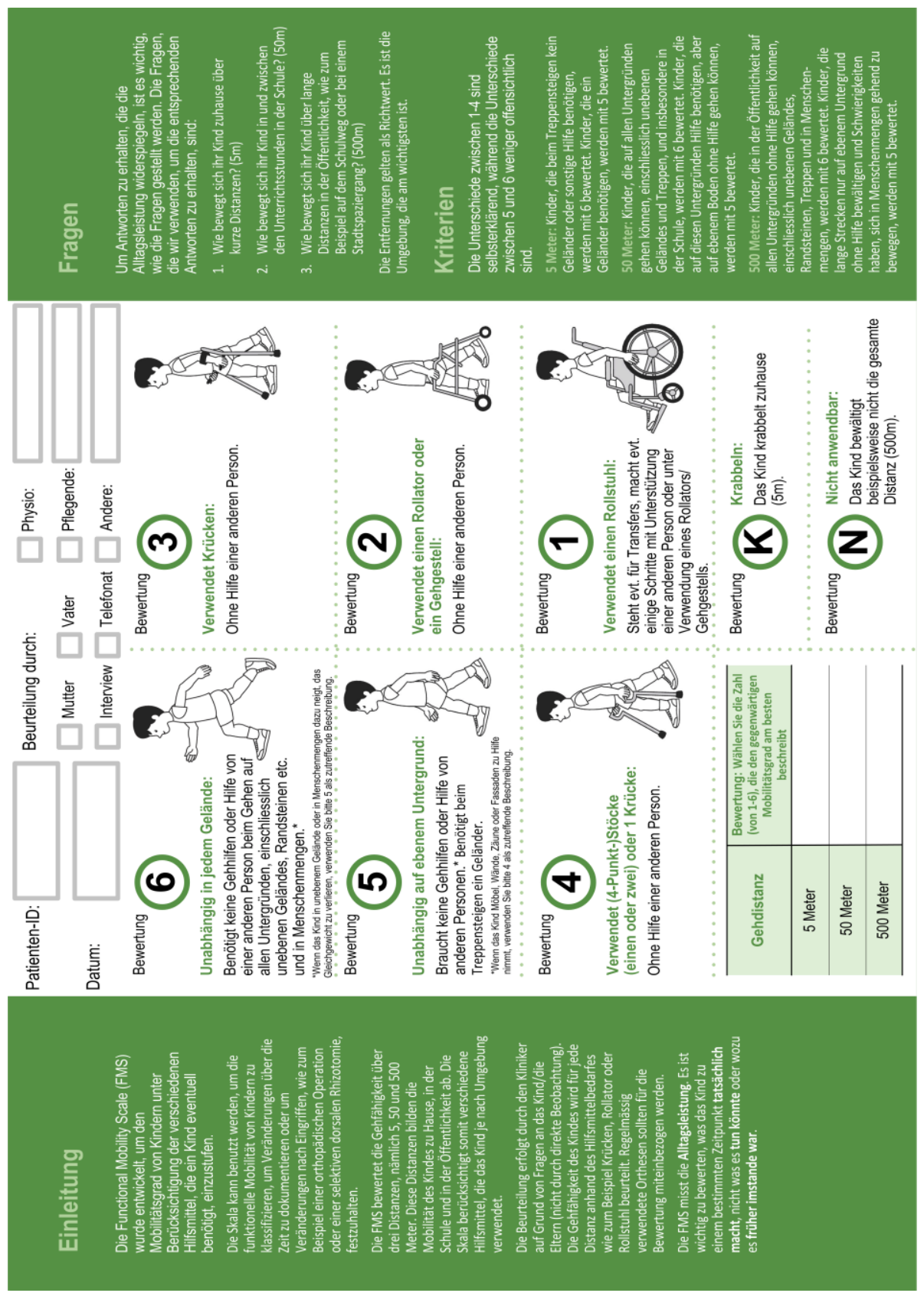


\section{GERMAN GILLETTE FUNCTIONAL ASSESSMENT QUESTIONNAIRE - WALKING SCALE}

\section{Gillette Functional Assessment Questionnaire Walking Scale}

\section{Fragebogen zur funktionellen Gang-Beurteilung}

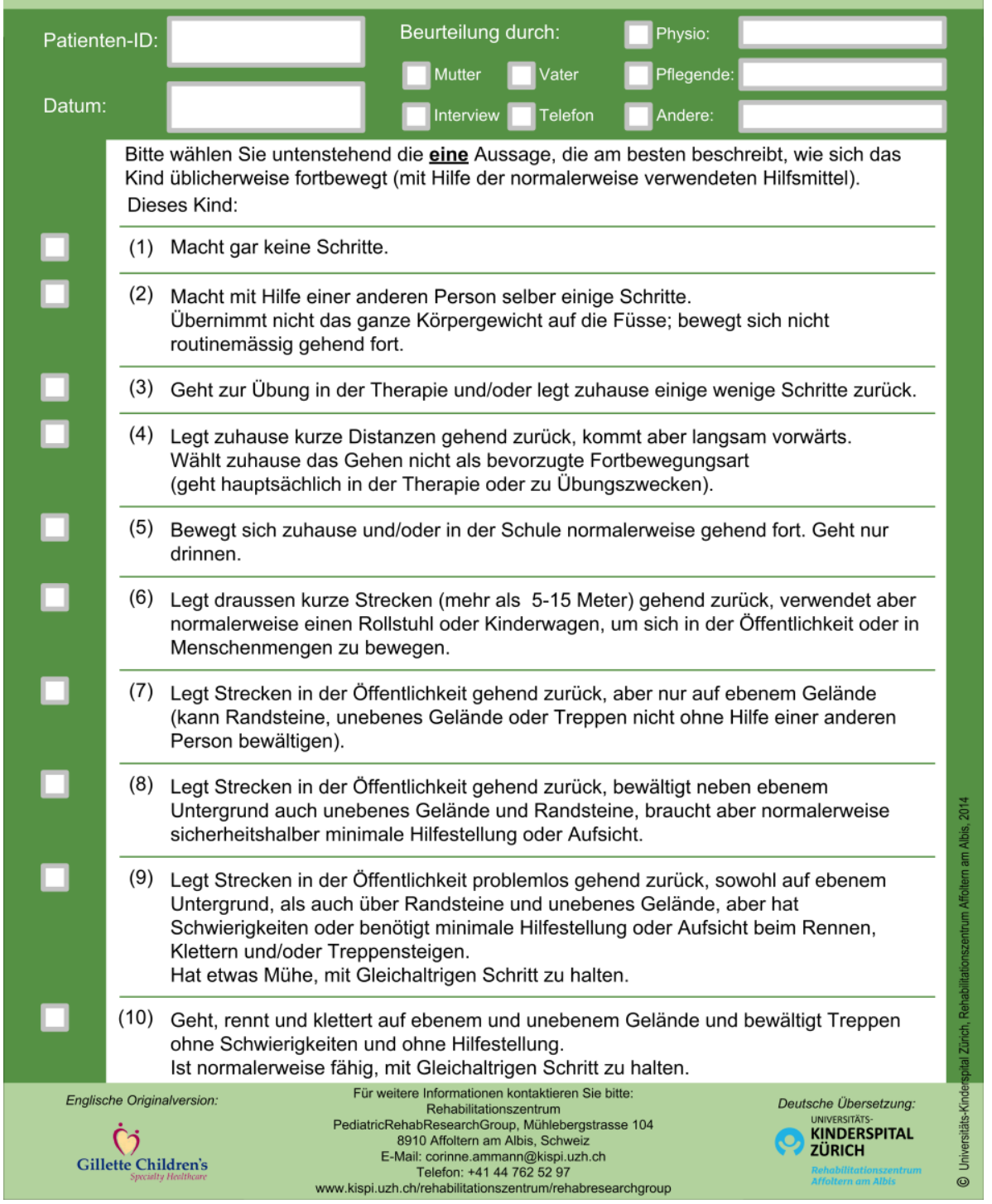




\section{SUMMARY}

Mobility, or the ability to move or be moved in one's environment with ease and without restrictions, is a relevant factor regarding physical independence and social participation. Many children with acquired or congenital neuromotor disorders are faced with limitations concerning their independent mobility, with restrictions in their walking abilities playing a vital role in this respect. Hence, an essential goal of neuropediatric rehabilitation is to influence children's walking abilities positively. This thesis focuses on two different areas that are closely interlinked within rehabilitation: the measurement and the training of functional walking abilities in children with neuromotor disorders.

CHAPTER 1 describes the significance of mobility and how the mobility of children with neuromotor disorders is affected. With the International Classification of Functioning, Disability and Health for Children and Youth (ICF-CY), it introduces an internationally accepted and widely used framework that facilitates a holistic view to document and measure a child's health and disability status and to plan or evaluate interventions in clinical and research settings. Cerebral palsy (CP) - with an incidence rate of 2-3 per 1'000 live births one of the most common congenital disabilities - and its consequences on various health dimensions and possible secondary impairments are discussed. The chapter also addresses the expanded and revised Gross Motor Function Classification System (GMFCS - E\&R), which can be considered an essential standard in clinical practice and research to classify the gross motor function of children with CP. It is based on categorizing self-initiated movements with an emphasis on sitting, walking, and wheeled mobility. Furthermore, the chapter gives an insight into the multidisciplinarity and the content of pediatric neurorehabilitation and discusses various treatment approaches affecting gait function. The evidence of these different gait interventions and their implementation modes is discussed with a particular focus on robot-assisted gait training (RAGT). Additionally, several aspects that should be considered when measuring walking ability are addressed: The influence of the environment, which is reflected in the different constructs of performance and capacity, and the 
psychometric properties of an outcome measure, which should be taken note of depending on the measurement goals. The chapter ends with the outline and the aims of this thesis, addressing the evaluation and the training of gait function. The aims were to:

(1) investigate and summarize the evidence of gait instruments and their psychometric properties in neuropediatric populations

(2) gain knowledge of the psychometric properties of selected measures reflecting functional mobility performance in our inpatient neurorehabilitation population

(3) evaluate the effectiveness of RAGT in children with CP.

CHAPTER 2 systematically investigates and summarizes the evidence of gait capacity and performance measures with the ICF-CY as a reference framework and evaluates their psychometric properties in neuropediatric populations. Our systematic review provided an overview of the current evidence on the psychometric properties of 27 measures used to assess functional gait in children with neuromotor disorders. CP was the diagnosis most often considered in studies examining the psychometric properties of a measure. Most studies evaluated aspects of reliability. Studies on measurement error and especially responsiveness were the rarest. The studies' sample sizes were generally low. Our review revealed a substantial lack of evidence regarding the psychometric properties of many measures that are regularly used in research and clinical practice. Furthermore, only a few of the appraised measures addressed the performance level of the ICF-CY, even though these measures take the influence of the environment children are exposed to in real life into account, which we consider very relevant.

Based on the findings of the systematic review and the underrepresentation of performance measures in our neurorehabilitation inpatient setting, we considered the Functional Mobility Scale (FMS) and the Functional Assessment Questionnaire - walking scale (FAQ) to be the most suitable tools to be applied in our setting, regarding their practicability and usability. Both are performance measures that address functional mobility in the daily environment. To be used in our rehabilitation center, we translated the FMS and FAQ into German and evaluated their 
psychometric properties in children and adolescents with neuromotor disorders, who were inpatients in our center.

CHAPTER 3 reports on the translation and the examined concurrent validity of these two measures, by comparing them to well-known activity measures assessing walking abilities in children, such as the walking item of the Functional Independence Measure for Children (WeeFIM walking), the 10-meter (10MWT) and 6-minute walk tests (6MinWT), and the dimension E (walking, running, jumping) of the Gross Motor Function Measure-88 (GMFM E). All correlation coefficients were moderate to strong, exceeded the hypothesized lower limits, and corresponded well with the reported values of the original English versions. However, correlations with the WeeFIM walking, which is also a performance measure, were not higher than correlations with the capacity measures. A possible explanation for this finding could be that the environment in the rehabilitation center with the optimized infrastructure and the daily routine of the inpatient setting may have reduced the differences between walking performance and walking capacity.

Both measures did not seem to be affected by any floor or ceiling effects in our patient sample, and none of the correlation coefficients exceeded 0.8 . Thus, the FMS and $F A Q$ provided additional information on functional mobility in the inpatient setting and facilitated a more differentiated picture of patients' mobility levels in varying contexts during the inpatient stay. Measuring performance hereby provides a meaningful perspective for the child and their family as the results are directly transferable to the child's behavior in everyday life.

Our study expands the already existing evidence of the FMS and FAQ's validity to assess functional mobility also in the inpatient setting.

CHAPTER 4 determines the interrater reliability of the FMS and FAQ. We investigated several sources of variation regarding the raters and the environment by comparing the ratings between nurses and physiotherapists in our inpatient neurorehabilitation setting and between these health professionals at one side and the parents' ratings in the home environment on the other side. We performed these comparisons twice; at the start and end of active gait rehabilitation. By choosing such a design - with different characteristics between the groups of raters, different 
settings, and two time points to investigate - we wanted to examine whether the scores of children's performance levels of functional mobility are comparable between a home and an inpatient setting despite differences in the environment and the raters' background. Furthermore, we wanted to know whether the timespan a health professional has worked with an individual child influences the rating.

With weighted kappa values of $0.69-0.77$ for the FAQ and $0.62-0.92$ for the FMS, interrater agreements between physiotherapists, nurses, and parents were above our predefined acceptance level of 0.6 at both time points. Our results indicate that functional mobility performance in the inpatient setting and the children's everyday environment seem to correspond well with each other and that all rater groups score the $F M S$ and $F A Q$ equally.

CHAPTER 5 explores the responsiveness and minimally important change (MIC) of the FMS and FAQ. For this purpose, we applied a construct-based approach (i.e., we examined the correlations between changes over time in FMS/FAQ scores to changes over time in standard functional gait measures) and a criterion-based approach (i.e., we introduced a 5-point ordinal global rating scale (GRS) to quantify the physiotherapists' perceived change of children's functional mobility performance after inpatient rehabilitation). Both methods are recommended by the COnsensusbased Standards for the selection of health Measurement INstruments (COSMIN) group. In line with these recommendations, we defined a priori hypotheses about the expected results. Regarding the construct approach, change scores of the FMS/FAQ correlated between 0.54-0.76 with the WeeFIM walking, the other performance measure, 0.35-0.49 with capacity measures like the 10MWT, 6MinWT, and GMFM $\mathrm{E}$, and 0.57-0.76 with the GRS. We dichotomized the GRS with the physiotherapists' perceived change of functional mobility performance (unchanged and improved) and used it as a criterion to perform receiver operating characteristic (ROC) curves analyses. The values of the area under the ROC curves (AUC), which were derived from this criterion approach, were above the preset acceptance level of 0.7 . Exceeding this limit demonstrated the measures' ability to accurately discriminate between children who did or did not improve according to the therapists' appraisal. 
Using the GRS, we further obtained a definition of what therapists consider as minimally important change. The MIC values indicated a 1-point increase for the FMS and a 2-point increase for the FAQ to be clinically meaningful.

In summary, we have demonstrated in chapters 3 to 5 that the FMS and FAQ are valid and reliable tools to assess the functional mobility performance of children with neuromotor disorders not only in their everyday home environment but also in the inpatient rehabilitation setting. Further, these measures can be used to detect changes in functional mobility performance that have occurred during inpatient rehabilitation, and thresholds were determined which define whether an observed change can be considered clinically meaningful.

CHAPTER 6 describes the study protocol and presents the theoretical considerations of a pragmatic crossover trial on the effectiveness of RAGT on the Lokomat compared to usual care. Children aged 6-18 years with bilateral spastic CP who could walk at least 14 meters with or without walking device were recruited. They were randomized to two different pre-specified sequences of interventions. One intervention was RAGT as treatment $(T)$ on the Lokomat in the outpatient setting of our rehabilitation center, consisting of three weekly sessions over five weeks. The other intervention was usual care as control (C) in the home environment of the child (consisting of 1-2 sessions of physiotherapy per week and additional hippotherapy, circuit training, and occupational therapy as necessary). A child could be randomized to a T/C sequence (TC-group) or a C/T/C sequence (CTC-group). Outcome measures included the GMFM $E$ as the primary outcome, and the dimension D (standing) of the GMFM-88 (GMFM D), as well as 6MinWT and 10MWT as secondary outcomes - assessed before and at the end of each intervention period. A sample size calculation indicated a sample size of 30 to be sufficient to detect a difference of 3.7\%-points in the dimension E-score of the GMFM-88. We increased this number to 34 to allow for a dropout rate of about $10 \%$ and planned a trial duration of four years.

CHAPTER 7 presents the findings of the pragmatic, randomized, crossover trial described in chapter 6 and reflects on the lessons we learned from conducting this trial. We stopped the study prematurely after more than eight years and after 16 of 
the planned 30 patients had finished the protocol. Our results indicated that five weeks of RAGT, applied as a standalone therapy intervention, were not superior to five weeks of usual care regarding the improvement of walking abilities in ambulatory children with spastic CP. Although these results may have been due to the underpowered study, the effect sizes of the primary, as well as the secondary outcome measures (except for the 6MinWT, which showed a small effect size), do not suggest any other conclusion.

Performing this trial, designed more than nine years ago as explained in chapter 6, has taught us a lot from a content-related as well as methodological point of view. We had aimed for a pragmatic trial design. However, the choices we made regarding the setting and recruitment turned out to be more on the explanatory side, which emerged as aggravating factors in the course of the study. Although we also provide outpatient therapy in our clinic, the RAGT intervention on the outpatient basis proved to be the main reason for our recruitment problems. Most families preferred to arrange the RAGT period during their child's school holidays, to limit the burden on their child due to therapy and travel expenses as much as possible. This fact reduced the number of participants considerably. Contrary to earlier conventions of 10 years ago, when we had planned our study, current clinical recommendations are to embed RAGT as an add-on therapy element within an individualized holistic treatment package. This approach also reflects the common practice of today's rehabilitation and thus contributes to producing research results that are relevant and applicable in practice. Considering performance in everyday life and addressing patients' personal goals when applying outcome measures are further factors to increase the relevance of research results for families and health professionals.

CHAPTER 8 summarizes the main findings, discusses methodological considerations, addresses implications for future research and clinical practice, and concludes with the main contributions of this thesis. We highlighted the lacking evidence on psychometric properties of measures assessing walking abilities in children with neuromotor disorders and the general shortage of performance measures regarding children's functional mobility in their daily environment. This thesis reduced the gap regarding both of these issues by providing evidence for 
aspects of validity, reliability, and responsiveness of the German versions of the FMS and $F A Q$ in the inpatient setting. Further, we facilitated the interpretation of change scores by defining MIC values for both measures.

As the crossover trial indicated that RAGT on an outpatient basis was not effective in improving walking abilities in ambulatory children with $\mathrm{CP}$, evidence on the effectiveness of pediatric RAGT is still vague. Because of the small sample sizes, heterogeneous patient populations, and special needs, intervention studies in the pediatric field remain challenging and ask for innovative research approaches. 


\section{ZUSAMMENFASSUNG}

Mobilität beschreibt die Fähigkeit, sich in seiner Umgebung leicht und ohne Einschränkungen zu bewegen oder bewegt zu werden. Sie ist ein relevanter Faktor für körperliche Unabhängigkeit und soziale Teilhabe. Viele Kinder mit erworbenen oder angeborenen neuromotorischen Beeinträchtigungen sind mit Restriktionen ihrer unabhängigen Mobilität konfrontiert. Hierbei spielen Einschränkungen in ihrer Gehfähigkeit eine wichtige Rolle. Ein wesentliches Ziel der neuropädiatrischen Rehabilitation ist es daher, die Gehfähigkeit der Kinder positiv zu beeinflussen. Diese Arbeit konzentriert sich auf zwei verschiedene Bereiche, die in der Rehabilitation trotzdem eng miteinander verknüpft sind: die Messung und das Training der funktionellen Gehfähigkeit bei Kindern mit neuromotorischen Beeinträchtigungen.

KAPITEL 1 beschreibt die Bedeutung von Mobilität und inwiefern Mobilität von Kindern mit neuromotorischen Störungen beeinträchtigt ist. Mit der Internationalen Klassifikation der Funktionsfähigkeit, Behinderung und Gesundheit bei Kindern und Jugendlichen (ICF-CY = International Classification of Functioning, Disability and Health - Children and Youth version) wird ein international anerkanntes und weit verbreitetes Rahmenkonzept eingeführt, das eine ganzheitliche Sichtweise zur Dokumentation und Messung des Gesundheits- und Behindertenstatus eines Kindes sowie zur Planung oder Bewertung von Interventionen in Klinik und Forschung ermöglicht. Zerebralparese ( $\mathrm{CP}=$ cerebral palsy) - mit einer Inzidenzrate von 2-3 pro 1'000 Lebendgeburten eine der häufigsten angeborenen Behinderungen - und ihre Auswirkungen auf verschiedene gesundheitliche Dimensionen und mögliche sekundäre Beeinträchtigungen werden diskutiert. Das Kapitel befasst sich ausserdem mit dem erweiterten und überarbeiteten Gross Motor Function Classification System (GMFCS - E\&R = expanded and revised), das als grundlegender Standard in der klinischen Praxis und Forschung zur Klassifizierung der Grobmotorik von Kindern mit CP angesehen werden kann. Der GMFCS - E\&R basiert auf der Kategorisierung selbstinitiierter Bewegungen mit Schwerpunkt auf Sitzen, Gehen und Mobilität auf Rädern. Darüber hinaus gibt das Kapitel einen 
Einblick in die Interdisziplinarität und den Inhalt der pädiatrischen Neurorehabilitation und diskutiert verschiedene Behandlungsansätze, die angewendet werden um die Gangfunktion zu beeinflussen. Die Evidenz dieser unterschiedlichen GangInterventionen und deren Umsetzung wird unter besonderer Berücksichtigung des robotergestützten Gangtrainings (RAGT = robot-assisted gait training) diskutiert. Zusätzlich werden einige Aspekte angesprochen, die bei der Messung der Gehfähigkeit berücksichtigt werden sollten: Der Einfluss der Umwelt, der sich in den verschiedenen Konstrukten von Performance und Kapazität widerspiegelt, und die psychometrischen Eigenschaften von Messinstrumenten, die man abhängig von den jeweiligen Messzielen beachten sollte. Das Kapitel endet mit der Gliederung und den Zielen dieser Arbeit:

(1) die Evidenz von Ganginstrumenten und ihren psychometrischen Eigenschaften in neuropädiatrischen Populationen zu untersuchen und zusammenzufassen.

(2) Kenntnisse zu gewinnen über die psychometrischen Eigenschaften ausgewählter Messinstrumente, die die funktionelle Mobilität in unserer stationären Neurorehabilitationspopulation auf Performance-Ebene widerspiegeln.

(3) die Wirksamkeit von RAGT bei Kindern mit CP zu beurteilen.

KAPITEL 2 untersucht und fasst die Evidenz von Messinstrumenten auf der Kapazitäts- und Performance-Ebene mit dem ICF-CY als Rahmenkonzept systematisch zusammen und bewertet deren psychometrische Eigenschaften in neuropädiatrischen Populationen. Unsere systematische Übersicht gibt einen Überblick über die aktuellen Erkenntnisse zu den psychometrischen Eigenschaften von 27 Messinstrumenten zur Beurteilung des funktionellen Gehens bei Kindern mit neuromotorischen Störungen: CP war die häufigste Diagnose in Studien, die die psychometrischen Eigenschaften eines Messinstrumentes untersuchten. Die meisten Studien bewerteten Aspekte der Reliabilität. Untersuchungen zu Messfehlern und vor allem zur Responsivität waren selten. Die Stichprobengrößen der Studien waren im Allgemeinen gering. Unsere Übersichtsarbeit zeigte einen erheblichen Evidenzmangel hinsichtlich der psychometrischen Eigenschaften vieler 
Messinstrumente, die regelmäßig in Forschung und klinischer Praxis eingesetzt werden. Darüber hinaus haben sich nur wenige der bewerteten Instrumente mit der Performance-Ebene des ICF-CY befasst, obwohl genau diese Messinstrumente den Einfluss der Umwelt berücksichtigen würden, dem Kinder im realen Leben ausgesetzt sind, was wir für sehr relevant halten.

Basierend auf den Ergebnissen unserer systematischen Übersichtsarbeit und der Unterrepräsentation von Performance-Instrumenten in unserer stationären Neurorehabilitation haben wir die Functional Mobility Scale (FMS) und die Functional Assessment Questionnaire - Walking Scale (FAQ) ausgewählt, da uns ihre Anwendung benutzerfreundlich und für unsere Umgebung praktikabel erschien. Beides sind Performance-Instrumente, die sich mit der funktionellen Mobilität im täglichen Umfeld befassen. Für den Einsatz in unserem Rehabilitationszentrum haben wir die FMS und FAQ ins Deutsche übersetzt und ihre psychometrischen Eigenschaften bei Kindern und Jugendlichen mit neuromotorischen Störungen, die in unserem Zentrum stationär behandelt wurden, evaluiert.

KAPITEL 3 berichtet über die Übersetzung und die untersuchte ÜbereinstimmungsValidität dieser beiden Messinstrumente, indem wir sie mit bekannten Aktivitätsinstrumenten verglichen, die die Gehfähigkeit von Kindern beurteilen, wie z.B. dem Gang-Item des Funktionellen Selbständigkeitsindex für Kinder (WeeFIM walking), dem 10-Meter- (10MWT) und 6-Minuten-Gangtest (6MinWT) und der Dimension E (Gehen, Rennen, Springen) der Gross Motor Function Measure-88 (GMFM E). Alle Korrelationskoeffizienten waren moderat bis stark, übertrafen die in Hypothesen formulierten Untergrenzen und stimmten gut mit den berichteten Werten der englischen Originalversionen überein. Die Korrelationen mit dem WeeFIM Walking, der ebenfalls ein Performance-Instrument ist, waren jedoch nicht höher als die Korrelationen mit den Kapazitätsinstrumenten. Eine mögliche Erklärung für dieses Resultat könnte sein, dass das Umfeld im Rehabilitationszentrum mit seiner optimierten Infrastruktur und dem Tagesablauf im stationären Bereich die Unterschiede zwischen Gang-Performance und Gang-Kapazität verringert haben könnte. 
Beide Messinstrumente schienen von keinem Boden- oder Deckeneffekt in unserer Patientenprobe betroffen zu sein, und keiner der Korrelationskoeffizienten lag über 0 ,8. Dies zeigt, dass FMS und FAQ zusätzliche Informationen zur funktionellen Mobilität im stationären Bereich lieferten und ein differenzierteres Bild des Mobilitätsniveaus der Patienten in den unterschiedlichen Bereichen während des stationären Aufenthaltes ermöglichten. Die Erhebung der Performance bietet dabei eine sinnvolle Perspektive für das Kind und seine Familie, da die Ergebnisse direkt auf das Verhalten des Kindes im Alltag übertragbar sind.

Unsere Studie erweitert die bereits vorhandene Evidenz betreffend Validität, um die funktionelle Mobilität auch im stationären Bereich mittels FMS und FAQ zu beurteilen.

KAPITEL 4 ermittelt die Interrater-Reliabilität der FMS und der FAQ. Wir untersuchten verschiedene Variations-Quellen in Bezug auf die Beurteilenden und das Umfeld, indem wir die Bewertungen zwischen Pflegefachpersonen und Physiotherapeuten in unserer stationären Neurorehabilitation und zwischen diesen Gesundheitsfachleuten auf der einen Seite und den Bewertungen der Eltern in der häuslichen Umgebung auf der anderen Seite verglichen. Diese Vergleiche führten wir zweimal durch: zu Beginn und am Ende der aktiven Gangrehabilitation. Durch die Wahl eines solchen Designs wollten wir feststellen, ob die Bewertung der funktionellen Mobilität von Kindern zwischen dem Umfeld zu Hause und in der stationären Rehabilitation vergleichbar ist - trotz Unterschieden in der Umgebung und dem Hintergrund der beurteilenden Personen. Ausserdem wollten wir wissen, ob die Zeitspanne, in der eine medizinische Fachperson mit einem einzelnen Kind gearbeitet hat, die Bewertung beeinflusst.

Mit gewichteten Kappa-Werten von 0,69-0,77 für die FAQ und 0,62-0,92 für die FMS lagen die Interrater-Übereinstimmungen zwischen Physiotherapeuten, Krankenschwestern und Eltern zu beiden Zeitpunkten über unserem definierten Akzeptanzgrenzwert von 0,6. Unsere Ergebnisse deuten darauf hin, dass die funktionelle Mobilität auf der Performance-Ebene im stationären Bereich und im alltäglichen Umfeld der Kinder gut miteinander übereinstimmen und dass die verschiedenen Beurteilenden die FMS und die FAQ gleich bewerten. 
KAPITEL 5 untersucht die Reaktionsfähigkeit und die minimal wichtige Veränderung (MIC = minimally important change) der FMS und der FAQ. Zu diesem Zweck verwendeten wir zwei verschiedene Ansätze. Einerseits untersuchten wir die Korrelationen zwischen den Veränderungen der FMS/FAQ-Scores und den Veränderungen in standardmässig angewendeten funktionellen Gangtests (Konstrukt-basierter Ansatz), andererseits quantifizierten wir die von den Physiotherapeuten wahrgenommene Veränderung der funktionellen AlltagsMobilität nach stationärer Rehabilitation der Kinder mittels einer 5-Punkte-Skala (GRS = global rating scale; Kriterium-basierter Ansatz). Beide Methoden werden von der COSMIN-Gruppe (COnsensus-based Standards for the Selection of health Measurement INstruments) empfohlen. Ebenfalls in Übereinstimmung mit diesen Empfehlungen definierten wir vorgängig Hypothesen über die zu erwartenden Ergebnisse. Die Veränderungen der FMS und der FAQ korrelierten zwischen 0,540,76 mit den Veränderungen des WeeFIM Walking, dem anderen PerformanceInstrument, zwischen 0,35-0,49 mit Kapazitätsinstrumenten wie 10MWT, 6MinWT und GMFM E und zwischen 0,57-0,76 mit der GRS. Die dichotomisierte GRS mit der von den Physiotherapeuten wahrgenommenen Veränderung der funktionellen Alltagsmobilität (unverändert und verbessert) verwendeten wir als Kriterium für die Durchführung von ROC-Kurvenanalysen $(\mathrm{ROC}=$ Receiver Operating Characteristics). Die Werte der Fläche unterhalb der ROC-Kurven (AUC = area under the ROC curve), die aus dieser Analyse abgeleitet wurden, lagen über der vorgegebenen Akzeptanzgrenze von 0,7. Die Überschreitung dieser Grenze zeigte die Fähigkeit der Messinstumente, Kinder, die sich nach Einschätzung der Therapeuten verbessert hatten oder nicht, genau zu unterscheiden.

Mit Hilfe der GRS erhielten wir außerdem eine Definition dessen, was Therapeuten als minimal wichtige Veränderung erachten. Die MIC-Werte wiesen darauf hin, dass für die FMS ein Anstieg um 1 Punkt und für die FAQ ein Anstieg um 2 Punkte als klinisch bedeutsam betrachtet werden kann.

Zusammenfassend haben wir in den Kapiteln 3 bis 5 gezeigt, dass FMS und FAQ valide und zuverlässige Instrumente sind, um die funktionelle Mobilität im Alltag von Kindern mit neuromotorischen Störungen nicht nur im heimischen Umfeld, sondern 
auch in der stationären Rehabilitation zu beurteilen. Darüber hinaus können mit diesen Messinstrumenten während der stationären Rehabilitation aufgetretene Veränderungen der funktionellen Alltags-Mobilität erkannt werden. Zudem wurden Grenzwerte bestimmt, welche definieren, ob eine beobachtete Veränderung als klinisch bedeutsam angesehen werden kann.

KAPITEL 6 beschreibt das Studienprotokoll und stellt die theoretischen Überlegungen einer pragmatischen Crossover-Studie zur Wirksamkeit von RAGT mit dem Lokomat im Vergleich zu Standardtherapie dar. Es wurden Kinder im Alter von 6-18 Jahren mit beidseitig spastischer CP rekrutiert, die mindestens 14 Meter mit oder ohne Hilfsmittel gehen konnten. Sie wurden in zwei verschiedene vordefinierte Interventionssequenzen randomisiert. Die eine Intervention war ambulantes RAGT mit dem Lokomat in unserem Rehabilitationszentrum als Behandlung ( $T=$ treatment), bestehend aus drei Einheiten pro Woche über fünf Wochen. Die andere Intervention war Standardtherapie als Kontrolle $(C=$ control $)$ in der häuslichen Umgebung des Kindes (bestehend aus 1-2 Sitzungen Physiotherapie pro Woche und zusätzlich Hippotherapie, medizinische Trainingstherapie und Ergotherapie nach Bedarf). Ein Kind konnte in eine T/C-Sequenz (TC-Gruppe) oder eine C/T/C-Sequenz (CTC-Gruppe) randomisiert werden. Die Ergebnisse umfassten die GMFM E als primären Endpunkt und die Dimension D (Stehen) der GMFM-88 (GMFM D) sowie 6MinWT und 10MWT als sekundäre Messinstrumente - bewertet jeweils vor und am Ende jedes Interventionszeitraumes. Eine Stichprobenberechnung ergab eine Stichprobengröße von 30, die ausreicht, um eine Differenz von 3,7\%-Punkten in der GMFM E zu ermitteln. Wir erhöhten diese Zahl auf 34 , um eine Abbruchsquote von etwa $10 \%$ zu ermöglichen, und planten mit einer Studiendauer von vier Jahren.

KAPITEL 7 präsentiert die Ergebnisse der in Kapitel 6 beschriebenen pragmatischen, randomisierten Crossover-Studie und reflektiert die Lehren, die wir aus der Durchführung dieser Studie gezogen haben. Wir haben die Studie nach mehr als acht Jahren vorzeitig abgebrochen, nachdem 16 der geplanten 30 Patienten das Protokoll abgeschlossen hatten. Unsere Ergebnisse zeigten, dass fünf Wochen RAGT, als eigenständige Therapie-Intervention angewendet, nicht 
wirksamer waren zur Verbesserung der Gehfähigkeit bei gehfähigen Kindern mit spastischer $\mathrm{CP}$ als fünf Wochen Standardtherapie. Obwohl diese Ergebnisse möglicherweise auf die zu schwache Fallzahl zurückzuführen sind, lassen auch die Effektgrößen der primären sowie der sekundären Endpunkte (mit Ausnahme des $6 \mathrm{MinWT}$, welcher eine geringe Effektgröße zeigte) keine andere Schlussfolgerung zu.

Die Durchführung dieser Studie, die, wie in Kapitel 6 erläutert, vor mehr als neun Jahren konzipiert wurde, hat uns sowohl inhaltich als auch methodisch viel gelehrt. Wir hatten ein pragmatisches Versuchsdesign angestrebt. Es zeigte sich jedoch, dass die Entscheidungen, die wir bezüglich Umfeld und Rekrutierung getroffen hatten, eher auf der explanatorischen Seite lagen, was sich im Laufe der Studie als erschwerender Faktor herausstellte. Obwohl wir in unserer Klinik auch ambulante Therapie anbieten, erwies sich die ambulante RAGT-Intervention als Hauptgrund für unsere Rekrutierungsprobleme. Die meisten Familien zogen es vor, die RAGTPeriode während der Schulferien ihres Kindes zu vereinbaren, um die auf das Kind wirkende Belastung durch Therapie und Reiseaufwand so gering wie möglich zu halten. Diese Tatsache reduzierte die Teilnehmerzahl erheblich. Entgegen den zum Zeitpunkt der Studienplanung geltenden Konventionen von vor 10 Jahren sind die aktuellen klinischen Empfehlungen, RAGT als zusätzliches Therapieelement in ein individuelles ganzheitliches Behandlungspaket einzubetten. Dieser Ansatz spiegelt auch die gängige Praxis der heutigen Rehabilitation wider und trägt so zu praxisrelevanten und anwendbaren Forschungsergebnissen bei. Die Berücksichtigung des Verhaltens im Alltag und der persönlichen Ziele der Patienten bei der Auswahl von Messinstrumenten sind weitere Faktoren, die die Relevanz von Forschungsergebnissen sowohl für Familien als auch für medizinisches Fachpersonal erhöhen.

KAPITEL 8 fasst die wichtigsten Ergebnisse zusammen, diskutiert methodische Überlegungen, befasst sich mit den Implikationen für zukünftige Forschung und klinische Praxis und schließt mit den wichtigsten Beiträgen dieser Arbeit ab. Wir haben die fehlende Evidenz betreffend der psychometrischen Eigenschaften von Messinstrumenten zur Beurteilung der Gehfähigkeit von Kindern mit 
neuromotorischen Beeinträchtigungen und den allgemeinen Mangel an Messinstrumenten, welche die funktionelle Mobilität von Kindern in ihrer Alltagsumgebung auf der Performance-Ebene erheben, hervorgehoben. Diese Doktorarbeit reduzierte die Lücke in diesen beiden Bereichen, indem sie Aspekte der Validität, Reliabilität und Responsivität der deutschen Versionen der FMS und der FAQ im stationären Bereich nachwies. Darüber hinaus haben wir durch das Definieren von MIC-Werten für beide Instrumente die Interpretation von erfassten Veränderungen erleichtert.

Da die Crossover-Studie zeigte, dass RAGT auf ambulanter Basis die Gehfähigkeit bei gehfähigen Kindern mit $\mathrm{CP}$ nicht verbessert, bleibt die Evidenz für die Wirksamkeit von pädiatrischer RAGT vage. Aufgrund der geringen Stichprobengröße, der heterogenen Patientenpopulationen und der besonderen Bedürfnisse bleiben Interventionsstudien im pädiatrischen Bereich anspruchsvoll und verlangen nach innovativen Forschungsansätzen. 


\section{VALORISATION}

\section{INTRODUCTION}

The term valorisation refers to the economic and societal impact that is created through the transfer of scientific knowledge. It is related to the term innovation, which describes the process of translating an idea or invention into a product or process that creates value. Thus, innovation only applies if an invention is put into practice. Similarly, researchers should strive to make scientific knowledge available to other organizations, interest groups or the general public. The valorisation addendum summarizes the societal and economic value of this dissertation besides the scientific aspects described in the previous chapters.

\section{RELEVANCE}

Mobility has become a buzzword in today's world. It is considered a prerequisite for a modern lifestyle. Mobility helps people to react quickly and flexibly and to move from one place to another without much effort. Being mobile is a requirement for achieving a goal, accomplishing something, finding one's way in life, and being worth something. With that, mobility is a significant criterion for human well-being.

Many children with neuromotor disorders are restricted in their independent mobility, which limits their possibilities to explore their environment actively and to interact with peers. Among the families concerned, their children's ability to walk is of particular importance. A vital part of rehabilitation is, therefore, to find the most effective interventions possible that have a positive impact on these walking abilities. By investigating the effectiveness of robot-assisted gait training (RAGT) in children with cerebral palsy, this dissertation has addressed one aspect from the wide range in this field.

Rehabilitation should further aim to enable children and their families to participate in society regardless of the children's mobility restrictions. It is, therefore, not enough to focus on improving walking capacity in the rehabilitation of gait disorders. To be precise, we need information that reflects children's mobility behavior in their everyday environment and should then address these individual circumstances in 
the rehabilitation process. With the Functional Mobility Scale (FMS) and the Gillette Functional Assessment Questionnaire - walking scale (FAQ), this dissertation provides two instruments for German-speaking countries, which allow obtaining specific information on the status and changes of children's everyday life mobility.

\section{TARGET GROUP}

The framework of this dissertation - both of the measuring and the training part - was designed from the very beginning to be relevant for everyday practice. Thus, many different stakeholders can benefit from the results in one way or another.

The knowledge gained from the measurement part of the thesis is expected to contribute to a reliable and valid measurement battery for centers like ours. Thanks to the systematic review, clinicians now have a detailed overview of all commonly used measurement instruments and their psychometric properties for the evaluation of gait function in children with neuromotor disorders. They can use this information as a decision-making basis to decide which instruments are most appropriate for their specific purposes. With the FMS and FAQ, various disciplines of healthcare professionals such as physical and sports therapists, nurses, and medical doctors have access to additional measurement instruments to evaluate the progress or their patients' walking abilities. The provided tools are particularly valuable as they reflect the children's mobility in everyday life and thus take into account the barriers and facilitators of their surrounding environment. Furthermore, they are easy and quick to use, which makes them ideal for everyday clinical use, where time resources are becoming increasingly scarce.

By reflecting children's walking abilities in their usual environment, the perspective of the two measurement instruments is particularly meaningful for the child and their families, as the ratings refer directly to everyday life. Another noteworthy aspect is that the measures enable a common language between parents and healthcare providers, which further empowers the parent's position in the healthcare system. In fact, the psychometric studies were themselves a factor that made children and their parents feel taken seriously as their usual environment and the parents' rating was decisive and thus highly valued. Moreover, many parents highly appreciated that 
they were contacted again by phone after the discharge of their child from the rehabilitation center.

While those mentioned above are the main stakeholders, the FMS and FAQ can also provide a more holistic view by considering the social and physical environment of the child regarding the communication with the health insurance companies when demonstrating the effectiveness of gait interventions. Furthermore, the instruments could also be implemented in various registries, such as the newly established Swiss cerebral palsy registry (www.swiss-cp-reg.ch).

Considering the training part of the dissertation, we discussed that a more holistic approach based on the International Classification of Functioning, Disability and Health (ICF), where RAGT is integrated into a whole treatment package, monitored by a sound measurement battery, is becoming more and more critical. Results of this thesis are supporting in finding an orientation for an improved basis for decisionmaking regarding optimal treatment for children and youth. This knowledge is not only of value for health care providers and health insurance companies but also meaningful for children with neuromotor disorders and their families. RAGT on the Lokomat requires a considerable effort from the child and their families regarding organization and time resources, as well as clarifying whether the health insurances will meet the costs. Gait training can take a smaller or more substantial part in rehabilitation, and RAGT can, but does not have to, be a part of it - depending on the individual needs and wishes of the children and their parents. Hopefully, our findings will remind families of the importance to carefully observe their child's progress and not merely blindly trust in the benefits of modern technologies. Besides, it was interesting to see how parents appreciated that interventions given to their child were questioned and scientifically examined. For this fact, they were also more than willing to invest the additional time required.

\section{ACTIVITIES, PRODUCTS, INNOVATION}

Before this dissertation, the FMS and FAQ were not yet available in German. Moreover, these two instruments reflect children's walking abilities in everyday life, which involves the influence of various environments that the children are exposed 
to in real life. This perspective - although particularly meaningful for the child and their family - is underrepresented in practice and research compared to the assessment of children's maximal walking capacity under standardized conditions. Finally, the FMS and FAQ are now some of the few pediatric functional mobility instruments with information on all their measurement properties, namely with positive evidence on their validity, interrater reliability, and responsiveness, and are easy and practical in use. Thus, clinicians and researchers can confidently apply both tools for the evaluation of children's walking abilities in everyday life.

RAGT with the Lokomat was implemented in our rehabilitation center more than ten years ago and is applied within our neuropediatric treatment regime in the out- as well as inpatient setting. Our outpatient program currently includes 20 RAGT sessions spread over 4-7 weeks, optionally accompanied by physiotherapy and sports lessons in the child's home setting. A comparable amount and intensity of sessions did not result in a measurable change in our intervention study that was part of this dissertation. These findings will therefore inevitably have to lead to a modification in the content of our RAGT outpatient program. The outpatient approach must be more oriented towards our 4-5 week-inpatient program, which already aims at gait training as a holistic package across all ICF dimensions with the aid of various therapeutic approaches. In a further step, the characteristics of the group to whom outpatient RAGT is recommended could be reconsidered to find those children that respond positively to this therapy method. Finally, an optimal assessment battery has to be assembled which allows recording the resulting changes at all ICF levels. As our center is one of the leading centers regarding robotic training in children, adaptations in our program will be noticed worldwide.

\section{SCHEDULE AND IMPLEMENTATION}

After the studies of this dissertation demonstrated positive evidence of their psychometric properties, the German FMS and FAQ have been implemented in the standard gait assessment of children performing RAGT on the Lokomat during their inpatient rehabilitation stay in our center. Additionally, they are becoming applied routinely in children undergoing a clinical 3-dimensional gait analysis in our gait 
laboratory. Furthermore, the physiotherapy team of our center has been instructed and trained in the use of the two instruments and has now started to rate functional mobility with the FMS and FAQ in their inpatients.

The proposed changes regarding the holistic RAGT outpatient approach need to be discussed with different stakeholders involved, including therapists responsible for RAGT in our clinic, rehabilitation physicians and most important the families to get their opinions and feedback about the feasibility. The impact of a modified approach should also be monitored to document changes carefully. This scientific support is imperative to have the health insurance companies on board as well to ensure coverage of the costs of this program, which should be based on the currently available best evidence. To minimize the risk of a noticeable improvement not being recognized at group level in a clinical trial, alternative pragmatic research designs that are suitable in this population, such as n-of-1 trials, single case methodology designs or observational designs could be viable options to be considered and explored in future research.

\section{TRANSFER OF KNOWLEDGE}

Besides the instruction of our physiotherapists and the implementation of the instruments in our center, we aim for as wide a distribution as possible of the German FMS and FAQ in the German-speaking part of Switzerland as well as later on also in other German-speaking countries. For this purpose, we already approached and will further contact relevant organizations in Switzerland. These are for example persons responsible for the training of physiotherapy students in the field of pediatrics and the master's degree in pediatric physiotherapy, therapy teams of other children's hospitals and schools for physically and multi-disabled children in the German-speaking part of Switzerland. We also offer interested organizations the opportunity to present the instruments and their application to the interested teams at their sites. The aim is to inform them about the German versions of these two instruments and to promote their implementation in clinical practice. Furthermore, we provide the tools on our website free of charge (www.kispi.uzh.ch/rza/de/forschende/publikationen/downloads). Additionally, we 
disseminated the results of our studies and distributed free copies of the German FMS and FAQ to interested people at various scientific conferences.

Related to the instruments' implementation in our rehabilitation center, an FMS and FAQ rating by the parents at their child's admission to the inpatient stay would be a medium-term vision to promote the common language between parents and healthcare providers and to largely underpin the perspective of parents in therapy goals and planning. Further medium-term goals are to implement the inclusion of FMS and FAQ into registries and other databases relating to pediatric neuromotor disorders to provide also information on children's motor performance at this level. 


\section{ACKNOWLEDGMENTS}

This thesis not only represents the studies it contains and my work at the computer, but it is also a milestone in more than a decade of work at the Rehabilitation Center of the University Children's Hospital Zurich (RCA) and specifically within the Pediatric Rehab Research Group (PRRG). At the RCA, I have been given the unique opportunity to start as a scientifically working physiotherapist when I was still in my postgraduate studies for my master's degree and continued working as a practical physiotherapist. Throughout this time, I got to know so many people who accompanied me on this journey and who supported me in a variety of ways. My thanks go to all these people.

Rob, I already met you during my Master's studies, and after my mixed experiences in your systematic review module, I vowed to never conduct a systematic review in real life... Thank you for giving me the opportunity to do an external PhD at your department, being my supervisor and leaving me as much freedom as I could have wished for. I am always fascinated by your extensive overview (even of Maastricht's historical background - as I learned recently), of how you can quickly grasp the essentials of a study, pose specific questions, and break down complex content in a way that everyone can understand it. Your feedback has always been clear and short, which is much the way I like it - as is your sense of humor.

Carolien, your appointment as my co-supervisor was a perfect match. Your professional past as a pediatric physiotherapist, the fact that you became a mother of three boys during your $\mathrm{PhD}$, and your interest and expertise in health measurements are almost incredibly coincident with my background (even though I am one child and a great deal of expertise behind compared to you). I have always thought of myself as an accurate person, but that is nothing compared to you... I appreciate your calmness, your sincere interest in my projects and your thoroughness with which you have been involved in them. Nevertheless, you have always shown understanding and backed me up when my priorities have been elsewhere. 
Judith, the third one of the Maastricht team. Thank you for always being so genuinely interested and actively involved in the discussions about my project at our $\mathrm{PhD}$ meetings in Winterthur, although you were not one of my supervisors.

Huub, we know each other since you came to Switzerland as a young PhD student, and helped out in our physio team in the Spinal Cord Injury Center at Balgrist University Hospital whenever there was help needed from a young, top motivated Dutchman. Luckily, our paths have crossed again almost ten years later at the RCA (with some help to make it happen...), when you were appointed as head of our PRRG. Although people say that we sometimes argue with each other like an old married couple, the trust and freedom you grant me and the support I receive from you when I need it are entirely unprecedented. You were the one who encouraged me to take on the challenge of a $\mathrm{PhD}$ (what else would I have done in the last six years?) and accompanied me during this phase as my local co-supervisor. This resulted in many joint jogging rounds (also called PhD-meetings), where, surprisingly, you always happened to ask your most critical questions just before the beginning of an ascent. You also endorsed me in my believes that health, family planning and family time in New Zealand have a priority even during a PhD. You have fought your way through countless of my never-ending sentences and kept my back free with cross-financing. You make me feel valuable for the team, no matter how often I am in the office. I feel privileged to work in your team and hope for many more years that we spend together at the RCA.

Beat and Andreas, you two were so visionary and initiated the first research position at the RCA. You believed in Physiotherapy Sciences and gave me as a greenhorn the opportunity to do and help establish research at our rehabilitation center. Unbelievable where we have since arrived thanks to your initiative, commitment, and dedication for the RCA and our research group.

I am thankful for the financial support that I received for my projects from the UBS Donation by order of a client, the Stiftung Cerebral, and the Mäxi Stiftung, Zurich, Switzerland, as well as the science awards from the Rheinfelden Rehabilitation Clinic, the Swiss Physiotherapy Association physioswiss, and the Anna Müller Grocholski Stiftung. 
Prof. Kerr H. Graham and Dr. Adrienne Harvey of the Royal Children's Hospital in Melbourne, Australia, and Dr. Tom F. Novacheck and Jean L.M.S. Stout of the Gillette Children's Specialty Healthcare in St. Paul, USA: a special thanks for your helpfulness and candor regarding our German translations of your original English versions of the Functional Mobility Scale and the Gillette Functional Assessment Questionnaire - walking scale. The cooperation with you was exemplary.

To all the members of the reading committee and the corona: Prof. Dr. R.J. Vermeulen, Dr. P. Heuts, Prof. Dr. K. Klingels, Prof. Dr. T. Lenssen, Dr. L.B. Mokkink, Dr. E.A.A. Rameckers, Prof. Dr. R.J.E.M. Smeets, thank you for your interest to evaluate my work and the time and effort you have invested.

Former (Annick, Corinna, Fabienne, Julia, Karin, Mischa, Nadine, Nina) and present members (Anja, Anne-Laure, Annina, Fabian, Jan, Jeff, Judith, Nicole, Petra, Rob, Sandra, Tabea, Urs, Volker) of the PRRG, you are just amazing. The diversity, multidisciplinarity, and open-mindedness are undoubtedly essential characteristics of our team, but our outstanding qualities are the deep respect for each other's abilities, the willingness to stand up for each other, reinforce one another, and rejoice at each other's successes. I always feel appreciated, respected, and accepted as a full member, no matter how often I am in the office. Although they make me feel older and older, our master students also play a decisive and essential role, continually bringing a fresh wind into our team, contributing their ideas, and taking care of their responsibilities with dedication and enthusiasm.

Judith, our ideologies, beliefs, and values are so similar in many respects. Work and efficiency are forgotten sometimes, as the discussions with you are so interesting and enriching. I enjoy having you as a teammate in our small-scale open space office.;-) It is great to know you are at my side as a paranymph on the day of my defense, thank you!

Corina, doing your master thesis within my performance measures project, you were an invaluable help in many aspects, and you contributed a lot to the success of my thesis.

Anke and Bernadette, my $\mathrm{PhD}$ mates from the first hour, we have shared many joint moments of worries, stress, and frustration. The possibility to exchange views and 
thoughts with you - not only related to our $\mathrm{PhD}$ projects - has always been soothing to me. Thank you, Bernadette, for being my paranymph.

A warm thank you to all the physios and nurses at the RCA. You had to deal with filling out all the questionnaires, endured my interviews, and tried to share (or at least tolerate) my enthusiasm for this matter.

To all the participating children and parents: I am deeply grateful for the time and effort you were willing to take on yourselves. Your willingness and the appreciation I have received from you have shown me that my work is meaningful to you - which is my utmost goal to achieve.

Oliver, thanks for the more than 100 entertaining English lessons we enjoyed together in the last years that helped me a lot to express myself without overthinking how to say it. You are always so well prepared (including your unmistakable old-style exercise sheets cut out and glued together by hand) and usually fill the lessons with excellent topics and worthwhile discussions. And thank you for holding back with writing tasks, even if it took some convincing.

Tom, you were my boss at the physiotherapy practice (the best practice you could ever wish to work in), where I had been working when I started my research career. You enabled me to do my Master's studies with a very flexible work schedule and gave me ideological and financial support. Your generosity, your openness, your enthusiasm, your calmness, your empathy, your never-ending urge to be active, your love for nature... It still hurts that you are not with us anymore.

I close by thanking my family, which is the most vital energy source of my life.

Mami und Papi, ihr habt mich gelehrt, für meine Bedürfnisse einzustehen und meinen Weg zu gehen. Ihr habt meiner Arbeit immer Interesse entgegengebracht und mich in meinen Plänen unterstützt, auch wenn sie manchmal für euch etwas abstrakt sein mochten. Mami, deine unzähligen flexiblen, immer wieder auch sehr spontanen Einsätze zum Jungs-Hüten waren und sind unglaublich wertvoll, nicht nur für mich, auch für die Jungs (und ich glaube auch für dich). Es ist schön, deinen Stolz zu spüren - aber dieses Buch musst du bitte trotzdem nicht allen unter die Nase halten...;-) 
Ursula und Heiri, hättet ihr nicht euren fixen wöchentlichen Grossmami/GrosspapiTag mit den Jungs, ich wäre wahrscheinlich auch 2020 noch nicht fertig mit dieser Doktorarbeit. Und dass ich dabei zum Teil auch gleich noch in den Genuss eines feinen Mittagessens komme, ist ja fast schon frech...

Grosi, unsere Beziehung zueinander war immer eine besondere und von Wertschätzung, Respekt und einer Wärme geprägt, die ich auch heute noch spüre, wenn ich an dich denke. Dass meine PhD-Defense nun genau an deinem Geburtstag stattfinden wird, betrachte ich als gutes Zeichen.

Cédric und Loric, ihr seid einfach die coolsten Jungs, die man sich wünschen kann (wenn ihr nicht gerade in einer schwierigen Phase steckt...;-)). Ihr sorgt dafür, dass ich immer wieder aufs Neue herausgefordert werde und die Bodenhaftung bestimmt nie verliere. Ihr bringt so viel Spannendes, Neues, Lustiges, Bereicherndes, Unerwartetes in mein Leben, so viele Emotionen, so viel Authentizität und Liebe ein Leben ohne euch ist für mich unvorstellbar.

Banya, Grisu, and Yuli, a life without pets is possible, but it is pointless. There is something so reassuring about spending time with you and petting you - I could do it all day long. Banya, you are my perfect personal trainer - without your paws on my back whenever I was sitting at the desk for too many hours, my sedentary behavior would have been even more excessive.

Martin, I know, a simple thank you would be enough in your eyes - if at all... But that would not do you any justice. I do not know who or where I would be without you. Your attitude to life, in which the family comes first when it matters, your unconditional support, your efforts to always find a way that suits all of us, your understanding, your open ear for all my worries - no matter how trivial they may be. Your ability to reflect on topics not familiar to you and to ask countless "stupid" questions. Your IT-support including hardware, software or just user knowledge. Our fabulous and unforgettable night-sessions filled with discussing optimal research designs, informative graphical illustrations and engaging presentations, developing databases, or creating Lego-constructions. Your love. Thank you! 


\section{ABOUT THE AUTHOR}

Corinne Ammann-Reiffer was born on 21 July 1975 in Schaffhausen, Switzerland. She studied Physiotherapy at the School of Physiotherapy of the University Hospital Zurich, Switzerland and received her physiotherapy diploma in 1999. She worked as a physiotherapist in the Spinal Cord Injury Center at the Balgrist University Hospital in Zurich, Switzerland and in a private physiotherapy practice, gaining practical experience in neurological and musculoskeletal rehabilitation, rheumatology and sports medicine. Besides many further education courses in these fields, she developed a particular interest in evidence-based practice. This interest and her attitude to challenge her treatment approaches critically led her to enter a part-time study program in Physiotherapy Science, which she completed with a Master's degree of the Maastricht University (NL) in 2006. In her thesis, she developed and evaluated a movement promotion program for primary school children. At the same time, Corinne started working as a research associate at the rehabilitation center of the University Children's Hospital Zurich in Affoltern am Albis, Switzerland, while she was still employed as a physiotherapist in a private practice. With the birth of her first son in 2007, she quit her practical job as a physiotherapist. Besides devoting herself to her family, which grew again in 2011 with the birth of her second son, she continued working part-time in the field of pediatric clinical research. Her main research interests are the evaluation of active therapeutic interventions and clinical assessments to assess gait function in pediatric neurorehabilitation. Corinne registered as an external $\mathrm{PhD}$ candidate at the Faculty of Health, Medicine and Life Sciences at Maastricht University in 2012 and completed the research described in this thesis under supervision of Prof. Rob de Bie, Dr. Caroline Bastiaenen, and Prof. Huub van Hedel. 


\section{LIST OF PUBLICATIONS}

Ammann-Reiffer C, Labruyère R. Letter to the Editor on "Effects of Antigravity Treadmill Training on Gait, Balance, and Fall Risk in Children With Diplegic Cerebral Palsy". Am J Phys Med Rehabil. 2018 Jun;97(6):e55-e56.

Ammann-Reiffer C, Bastiaenen CHG, Klöti C, van Hedel HJA. Concurrent Validity of Two Gait Performance Measures in Children with Neuromotor Disorders. Phys Occup Ther Pediatr. 2018 Jan 16:1-12. [Epub ahead of print]

Ammann-Reiffer C, Bastiaenen CHG, de Bie RA, van Hedel HJA. Interrater reliability of two gait performance measures in children with neuromotor disorders across two different settings. Dev Med Child Neurol. 2017 Nov;59(11):1158-1163.

Ammann-Reiffer C, Bastiaenen $\mathrm{CH}$, Meyer-Heim AD, van Hedel HJ. Effectiveness of robot-assisted gait training in children with cerebral palsy: a bicenter, pragmatic, randomized, cross-over trial (PeLoGAIT). BMC Pediatr. 2017 Mar 2;17(1):64.

Ammann-Reiffer C, Bastiaenen CHG, de Bie RA, van Hedel HJA. Measurement properties of gait-related outcomes in youths with neuromuscular diseases: a systematic review. Phys Ther. 2014;94:1067-1082. Epub 2014 May 1.

Borggraefe I, Dabrowski E, Schaefer J, Klaiber M, Ammann-Reiffer C, Knecht B, Berweck St, Heinen F, Meyer-Heim A. Dose dependent improvement of walking performance after robotic-assisted treadmill therapy in children and adolescents with cerebral palsy. Eur J Paediatr Neurol. 2010 Nov;14(6):496-502. Epub 2010 Feb 6.

Eugster Buesch F, Schlaepfer B, de Bruin ED, Wohlrab G, Ammann-Reiffer C, Meyer-Heim A. Constraint induced movement therapy (CIMT) for children with obstetric brachial plexus palsy - two single case series. Int J Rehab Res. 2010 Jun;33(2):187-92. Epub 2009 Sep 4.

Meyer-Heim A, Ammann-Reiffer C, Schmartz A, Schäfer J, Sennhauser FH, Heinen F, Knecht B, Dabrowski E, Borggraefe I. Improvement of walking abilities after robotic-assisted locomotion training in children with cerebral palsy. Arch. Dis. Child. 2009;94;615-620. Epub 2009 Feb 10.

Meyer-Heim A, Borggraefe I, Ammann-Reiffer C, Berweck St, Sennhauser FH, Colombo $G$ Knecht B, Heinen F. Feasiblity of Robotic assisted locomotor training in children with central gait impairment. Dev Med Child Neurol. 2007;49:900-6. 